Decomposição celular e torção de Reidemeister para formas espaciais esféricas tetraedrais 



\title{
Decomposição celular e torção de Reidemeister para formas espaciais esféricas tetraedrais
}

\author{
Ana Paula Tremura Galves
}

Orientador: Prof. Dr. Mauro Flávio Spreafico

Coorientador: Prof. Dr. Oziride Manzoli Neto

Tese apresentada ao Instituto de Ciências Matemáticas e de Computação - ICMC-USP, como parte dos requisitos para obtenção do título de Doutor em Ciências - Matemática . VERSÃO REVISADA 
Ficha catalográfica elaborada pela Biblioteca Prof. Achille Bassi e Seção Técnica de Informática, ICMC/USP, com os dados fornecidos pelo(a) autor(a)

\begin{tabular}{|c|c|}
\hline \multirow[t]{3}{*}{ G182d } & $\begin{array}{l}\text { Galves, Ana Paula Tremura } \\
\text { Decomposiça celular e torção de Reidemeister para } \\
\text { formas espaciais esféricas tetraedrais / Ana Paula } \\
\text { Tremura Galves; orientador Mauro Flávio Spreafico; } \\
\text { co-orientador Oziride Manzoli Neto. -- São Carlos, } \\
2013 . \\
\quad 154 \text { p. }\end{array}$ \\
\hline & $\begin{array}{l}\text { Tese (Doutorado - Programa de Pós-Graduação em } \\
\text { Matemática) -- Instituto de Ciências Matemáticas e } \\
\text { de Computação, Universidade de São Paulo, } 2013 .\end{array}$ \\
\hline & $\begin{array}{l}\text { 1. grupo binário tetraedral. 2. formas espaciais } \\
\text { esféricas. 3. região fundamental. } 4 \text {. decomposição } \\
\text { celular.5. torção de Reidemeister. I. Spreafico, } \\
\text { Mauro Flávio, orient. II. Manzoli Neto, Oziride, } \\
\text { co-orient. III. Título. }\end{array}$ \\
\hline
\end{tabular}


Aos meus pais,

José Rubens e Carlota,

e à minha avó Palmira, com amor, admiração e gratidão. 



\section{Agradecimentos}

Agradeço, primeiramente, à Deus pela constante presença em todos os momentos de minha vida, iluminando, protegendo e conduzindo os meus passos.

Aos meus pais José Rubens e Carlota pelo amor, carinho e pelo apoio incondicional que sempre me dedicaram. À minha avó Palmira pelo carinho, preocupação e orações. Ao meu irmão José Ricardo pela amizade e carinho. À todos os meus familiares que, tenho certeza, torceram muito para que este trabalho se concretizasse.

Ao meu noivo Vinícius, com amor e gratidão por sua compreensão, carinho, presença e incansável apoio ao longo desses quatro anos de doutorado.

Ao Prof. Dr. Mauro Flávio Spreafico por ter projetado este trabalho e pelos conhecimentos transmitidos. Em especial, ao Prof. Dr. Oziride Manzoli Neto pela convivência, pela paciência, pelos conhecimentos transmitidos e por sua solicitude quando sua ajuda se mostrou indispensável.

À minha amiga Lígia, pela participação no projeto e por tornar melhores os momentos de estudos e discussões, dando força e me apoiando sempre, mesmo nos momentos de desânimo e dificuldades.

À Prof ${ }^{\mathrm{a}}$ Dr $^{\mathrm{a}}$ Maria Gorete Carreira Andrade pela amizade e por me apresentar o caminho da pesquisa. Aos professores Denise de Mattos, Eduardo Tengan e Paulo Leandro Dattori pela convivência nesses quatro anos de doutorado e pela prestatividade em ajudar sempre.

Aos meus amigos Aldício, Marjory, Nazira e Taciana pela grande amizade que construímos e pelos momentos maravilhosos que passamos. Aos meus amigos de pós-graduação Alex, Alisson, Amanda, Giuliano, Matheus, Nelson, Northon, Renato, Suzete, Thaís e Vinícius, por tornarem horas tão difíceis de estudos, em momentos de pura alegria e 
descontração. À Francielle pelo carinho e amizade. E como não poderia deixar de faltar, aos meus amigos Amanda e Edinho, por terem tornado minhas viagens semanais mais divertidas e inesquecíveis.

À todos os funcionários do ICMC pela disponibilidade em ajudar sempre.

Ao $\mathrm{CNPq}$ pelo apoio financeiro.

E por fim, à todos que colaboraram, direta ou indiretamente, para a concretização deste trabalho. 
"Para o otimista, cada nova complicação é uma nova oportunidade. Para o pessimista, cada nova oportunidade é uma nova complicação."

(Içami Tiba) 



\section{Resumo}

Dada uma ação isométrica livre do grupo binário tetraedral $G$ sobre esferas de dimensão ímpar, obtemos uma decomposição celular finita explícita para as formas espaciais esféricas tetraedrais, fazendo uso do conceito de região (ou domínio) fundamental. A estrutura celular deixa explícita uma descrição do complexo de cadeias sobre o grupo G. Como aplicações, utilizamos o complexo de cadeias e a interpretação geométrica do produto cup para calcular o anel de cohomologia da forma espacial esférica tetraedral em dimensão três, e também calculamos a torção de Reidemeister destes espaços para uma determinada representação de $G$.

Palavras chave: grupo binário tetraedral, formas espaciais esféricas, região fundamental, decomposição celular, anel de cohomologia, torção de Reidemeister. 



\section{Abstract}

Given a free isometric action of a binary tetrahedral group $G$ on odd dimensional spheres, we obtain an explicit finite cellular decomposition of the tetrahedral spherical space forms, using the concept of fundamental domain. The cellular structure gives an explicit description of the associated cellular chain complex over the group $G$. As applications we use the chain complex and the geometric interpretation of the cup product to calculate the cohomology ring of the tetrahedral spherical space form in three dimension, and also compute the Reidemeister torsion of these spaces for a determined representation of $G$.

Keywords: binary tetrahedral group, spherical space forms, fundamental domain, cellular decomposition, cohomology ring, Reidemeister torsion. 



\section{Sumário}

Introdução

1 Nocões preliminares e formas espaciais esféricas

1.1 Acão de grupos e resolucões projetivas e livres . . . . . . . . . . . . . . . . . 19

1.2 Produto direto e semidireto . . . . . . . . . . . . . . . . . . . 21

1.3 Representacão de grupos finitos . . . . . . . . . . . . . . . . . . . 22

1.4 Classificacão dos grupos livres de ponto fixo $\ldots \ldots$. . . . . . . . . . . . . . . . . 29

1.5 Classificação das formas espaciais esféricas . . . . . . . . . . . . . . . . 32

2 Formas espaciais esféricas tetraedrais 33

2.1 Grupo binário tetraedral e algumas notacões . . . . . . . . . . . . . . . . . 33

2.1.1 Acões livres sobre esferas . . . . . . . . . . . . . . . . . . . 34

2.1 .2 Curved join $\ldots \ldots \ldots \ldots \ldots \ldots \ldots$. . . . . . . . . . . . . . . . . . . .

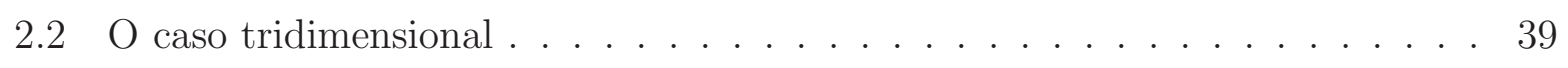

2.2 .1 A região fundamental . . . . . . . . . . . . . . . . . . . . . . 39

2.2.2 Decomposicão celular e complexo de cadeias . . . . . . . . . . . 55

2.3 O caso $4 n-1$ dimensional, $n>1 \ldots \ldots \ldots \ldots$

3 Grupos de homologia das formas espaciais esféricas tetraedrais $\quad 77$

3.1 Homologia com coeficientes locais . . . . . . . . . . . . . . 77

3.2 Grupos de homologia de $\mathscr{P}_{2} \ldots \ldots \ldots \ldots$. . . . . . . . . . 79

3.2.1 Grupos de homologia com coeficientes em $\mathbb{Z}$. . . . . . . . . . . . . 80

3.2.2 Grupos de homologia com coeficientes em $\mathbb{Z}_{2}$. . . . . . . . . . . . . . 84 
3.2.3 Grupos de homologia com coeficientes em $\mathbb{Z}_{3} \ldots \ldots$. . . . . . . . 87

3.2.4 Grupos de homologia com coeficientes em $\mathbb{Z}_{p}$. com $p$ primo e $p>3$. 91

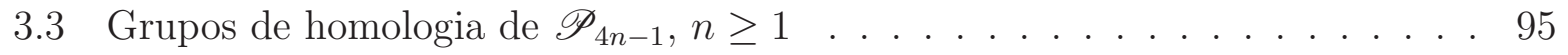

3.3.1 Grupos de homologia com coeficientes em $\mathbb{Z}$. . . . . . . . . . . . 95

3.3.2 Grupos de homologia com coeficientes em $\mathbb{Z}_{2}$. . . . . . . . . . . . . 96

3.3.3 Grupos de homologia com coeficientes em $\mathbb{Z}_{3}$. . . . . . . . . . . . . . 98

3.3.4 Grupos de homologia com coeficientes em $\mathbb{Z}_{p}$, com $p$ primo e $p>3 \quad$. 99

4 Anel de cohomologia das formas espaciais esféricas tetraedrais $\quad 101$

4.1 Módulo de homomorfismos . . . . . . . . . . . . . . . . . . . . . . . . . . . . 101

4.2 Produto Cup . . . . . . . . . . . . . . . . . . . . 104

4.2.1 Interpretacão geométrical . . . . . . . . . . . . . . . . . . . 105

4.3 Grupos de cohomologia de $\mathscr{P}_{2} \ldots \ldots \ldots$. . . . . . . . . . . . . . 107

4.3.1 Grupos de cohomologia com coeficientes em $\mathbb{Z}$. . . . . . . . . . 108

4.3.2 Grupos de cohomologia com coeficientes em $\mathbb{Z}_{2}$. . . . . . . . . . 108

4.3.3 Grupos de cohomologia com coeficientes em $\mathbb{Z}_{2}$. . . . . . . . . . . 109

4.3.4 Grupos de cohomologia com coeficientes em $\mathbb{Z}_{p}$, com $p$ primo e $p>3110$

4.4 Anel de cohomologia de $\mathscr{P}_{2} \ldots \ldots \ldots \ldots \ldots$

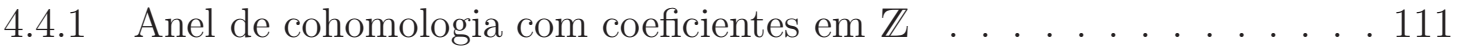

4.4 .2 Anel de cohomologia com coeficientes em $\mathbb{Z}_{2} \ldots \ldots$. . . . . . . . . 112

4.4.3 Anel de cohomologia com coeficientes em $\mathbb{Z}_{3}$. . . . . . . . . . . . . 112

4.4.4 Anel de cohomologia com coeficientes em $\mathbb{Z}_{p}$, com $p$ primo e $p>3$. . 114

4.5 Homotopia contrátil . . . . . . . . . . . . . . . . . . . . . . . 115

5 Cálculo da torcão de Reidemeister 129

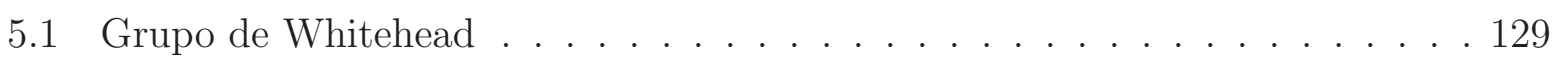

5.2 Álgebra dos quatérnios . . . . . . . . . . . . . . . . . . . . . 131

5.3 Torcão de Reidemeister de um complexo de cadeias . . . . . . . . . . . . 133

5.4 Cálculo da torção de Reidemeister das formas espaciais esféricas tetraedrais . 134

$\begin{array}{lr}\text { Referências Bibliográficas } & 149\end{array}$

$\begin{array}{ll}\text { Índice Remissivo } & 153\end{array}$ 


\section{Introdução}

As formas espaciais esféricas são obtidas como o espaço quociente das esferas (de dimensão ímpar) por ações livres de ponto fixo de grupos finitos; tais grupos são conhecidos. Estas são variedades Riemannianas conexas completas de curvatura constante positiva e o seu recobrimento universal é a esfera. O problema das formas espaciais esféricas subdividese em duas partes: uma delas é a de descrever os grupos onde tais ações podem ocorrer, e a outra é a de descrever como tais grupos podem agir. Ambas as partes dependem essencialmente da teoria de representação de grupos finitos. A divisão em duas partes foi observada por Vincent [27, porém a maior parte do trabalho na busca de tais grupos foi feita por Zassenhaus [29], o qual fez uso de resultados de teoria de grupos e teoria de representação.

A principal referência deste trabalho é o livro do Wolf [28] que apresenta uma classificação completa das formas espaciais esféricas. Wolf as classificou em seis tipos onde os quatro primeiros tipos envolvem grupos solúveis e os dois últimos, grupos não solúveis. Nesse trabalho, estaremos interessados nas formas espaciais esféricas tetraedrais, onde o grupo em questão é o binário tetraedral, o qual é solúvel.

Em [25] Tomoda e Zvengrowski estudaram os anéis de cohomologia da forma espacial esférica em dimensão três. A ideia básica é obter uma resolução explícita para o grupo fundamental $\pi$ de tais espaços (esses grupos são explicitamente conhecidos). Encontrar essas resoluções é um problema bastante complicado em topologia algébrica. O caso abeliano, isto é, $\pi$ um grupo cíclico, foi estudado em vários trabalhos de De Rham e Seifert. O seguinte caso investigado foi o grupo dos quatérnios generalizados. Uma resolução para tais grupos pode ser encontrada, por exemplo, em [6, Chap.XII, Section 7], porém nenhuma prova foi apresentada. Seguindo esta linha de raciocínio, Tomoda e 
Zvengrowski apresentaram resoluções explícitas para os grupos quatérnios generalizados, binário tetraedral, octaedral e icosaedral, com homotopias contráteis dadas explicitamente, fazendo uso de uma ferramenta computacional o GAP (Groups, Algorithms, Programming - um sistema para álgebra discreta computacional). Eles também apresentaram a estrutura de anel de outras duas famílias de grupos, os grupos split metacíclicos e os grupos binários tetraedrais generalizados, usando também o GAP.

A ideia deste trabalho é apresentar uma resolução para o grupo binário tetraedral, fazendo uso de outra ferramenta, a geometria. Nossa abordagem é baseada na ideia original de Swan [24] que consiste no seguinte: seja $G$ um grupo finito agindo livremente sobre uma esfera de dimensão $n\left(S^{n}\right)$, afim de obter uma resolução para o grupo $G$ é suficiente obter uma decomposição celular $G$-equivariante para $S^{n}$. O problema em aplicar tal ideia é computacional, ou seja, os cálculos são extremamente difíceis, e esta é a razão pela qual mesmo obtendo sucesso nos cálculos para os grupos cíclicos, esta ideia acabou abandonada. Em [16], foram estudados os grupos quatérnios generalizados, e em [11], foram estudados os grupos split metacíclicos. Em particular, seguimos a ideia introduzida por Cohen [7]. Em seu trabalho ele considera os grupos cíclicos, e uma sofisticada decomposição celular é obtida usando a decomposição de esferas no 'join' de algumas esferas de dimensões mais baixas, ou seja, o método utilizado para obter tal decomposição é essencialmente geométrico.

Aprimorando as técnicas do Cohen e utilizando o conceito de região (ou domínio) fundamental, obtemos uma decomposição celular para as esferas de dimensão ímpar, equivariante em relação ao grupo binário tetraedral, e dessa forma, apresentamos uma resolução livre de $\mathbb{Z}$ sobre $\mathbb{Z}[G]$, onde $G$ denota o grupo em questão.

Algumas das aplicações deste trabalho são os cálculos do anel de cohomologia e da torção de Reidemeister. A seguir, descrevemos alguns conceitos necessários para esses cálculos.

Quando estudamos a teoria de homotopia de espaços que não são simplesmente conexos, devemos considerar uma ação do grupo fundamental sobre algum grupo abeliano. Sistemas com coeficientes locais são uma ferramenta para organizarmos essa informação. A teoria torna-se mais complicada pelo fato de termos que considerar anéis não comutativos.

Existem duas abordagens para a construção do complexo que nos permite calcular a homologia e cohomologia com coeficientes locais de um espaço. A primeira é mais algébrica e tem como ponto de vista que o complexo de cadeias que será associado a um espaço $X$ 
é um complexo singular (ou celular) da cobertura universal $\widetilde{X}$, visto como um complexo de cadeias sobre o anel de grupos $\mathbb{Z}\left[\pi_{1}(X)\right]$. Deste ponto de vista, coeficientes locais nada mais são do que módulos sobre o anel de grupos $\mathbb{Z}\left[\pi_{1}(X)\right]$.

A segunda abordagem é mais topológica; consideramos um sistema de coeficientes locais sobre $X$ (ou seja, um fibrado sobre $X$ cujas fibras são grupos abelianos e cujas funções transição tomam valores nos automorfismos do grupo estrutural do fibrado) e definimos um complexo de cadeias considerando as cadeias como somas formais de simplexos singulares (ou celulares), com os coeficientes de um simplexo sendo um elemento da fibra sobre aquele simplexo (por isso a terminologia coeficientes locais). Porém, não abordamos tal construção e sim apenas a primeira.

Uma das vantagens da teoria de cohomologia é possuir uma estrutura multiplicativa, conhecida como produto cup, que transforma a cohomologia $H^{*}(X)$ do espaço $X$ em um anel (graduado) associativo, comutativo e com unidade, ou seja, transforma o grupo de cohomologia em um anel de cohomologia.

A torção de Reidemeister foi definida primeiramente para complexos simpliciais finitos por K. Reidemeister [20] e W. Franz [12], com o objetivo de classificar os espaços lenticulares. Estes, por sua vez, foram os primeiros exemplos de variedades que possuem o mesmo tipo de homotopia sem serem homeomorfos. Tais espaços foram completamente classificados por meio da torção de Reidemeister. Desta forma, a torção de Reidemeister foi o primeiro invariante de uma variedade que não é invariante por tipo de homotopia. Com isto, pode-se entender que a torção de Reidemeister consegue identificar a estrutura de interações entre o grupo fundamental e a estrutura simplicial.

O trabalho é dividido em cinco capítulos e seus conteúdos serão descritos resumidamente a seguir.

No primeiro capítulo, apresentamos alguns conceitos preliminares como ação de grupos e produto semidireto que serão bastante utilizados no decorrer do trabalho. Também apresentamos algumas definições e resultados que fornecerão os possíveis grupos que ocorrem na construção das formas espaciais esféricas, cuja referência principal é [28].

O capítulo seguinte é o principal da tese. Através de alguns conceitos algébricos e geométricos, obtemos resultados interessantes para a teoria das formas espaciais esféricas. Tal capítulo inicia-se com a apresentação do grupo binário tetraedral e suas relações. 
Estudamos a ação deste grupo nas esferas de dimensão ímpar $\left(S^{4 n-1}\right)$ e, usamos a geometria para encontrar uma decomposição celular desse espaço, determinando assim, uma região (ou domínio) fundamental e um complexo de cadeias coerente com tal ação, afim de lidarmos com o espaço quociente, que é chamado forma espacial esférica tetraedral e, dessa forma, obtemos uma resolução livre de $\mathbb{Z}$ sobre $\mathbb{Z}\left[P_{24}\right]$, onde $P_{24}$ denota o grupo binário tetraedral.

No terceiro capítulo utilizamos o complexo de cadeias, construído no capítulo anterior, para calcular os grupos de homologia (com coeficientes locais) das formas espaciais esféricas tetraedrais com coeficientes em $\mathbb{Z}, \mathbb{Z}_{2}, \mathbb{Z}_{3}$ e $\mathbb{Z}_{p}$, com $p$ primo e $p>3$. Tais grupos serão necessários para o cálculo dos grupos de cohomologia (com coeficientes locais) e, através da interpretação geométrica do produto cup, calculamos o anel de cohomologia da forma espacial esférica tetraedral em dimensão três, os quais apresentamos no quarto capítulo. Para dimensões maiores não é possível utilizar esta técnica geométrica, tornando-se necessário a definição da homotopia contrátil e, consequentemente, da aplicação diagonal. Devido a complexidade da homotopia contrátil não foi possível finalizar os cálculos da aplicação diagonal, tornando-se um projeto futuro.

O objetivo do último capítulo é apresentar o cálculo da torção de Reidemeister para as formas espaciais esféricas tetraedrais, utilizando uma dada representação do seu grupo fundamental que defina um complexo (com coeficientes locais) que seja acíclico.

Alguns conceitos básicos de topologia algébrica serão necessários tais como: grupos de homologia, complexo de cadeias, espaço de recobrimento, entre outros, os quais serão omitidos porém, podem ser encontrados em livros básicos de topologia algébrica como, por exemplo, em [3] e [8], [18]. 


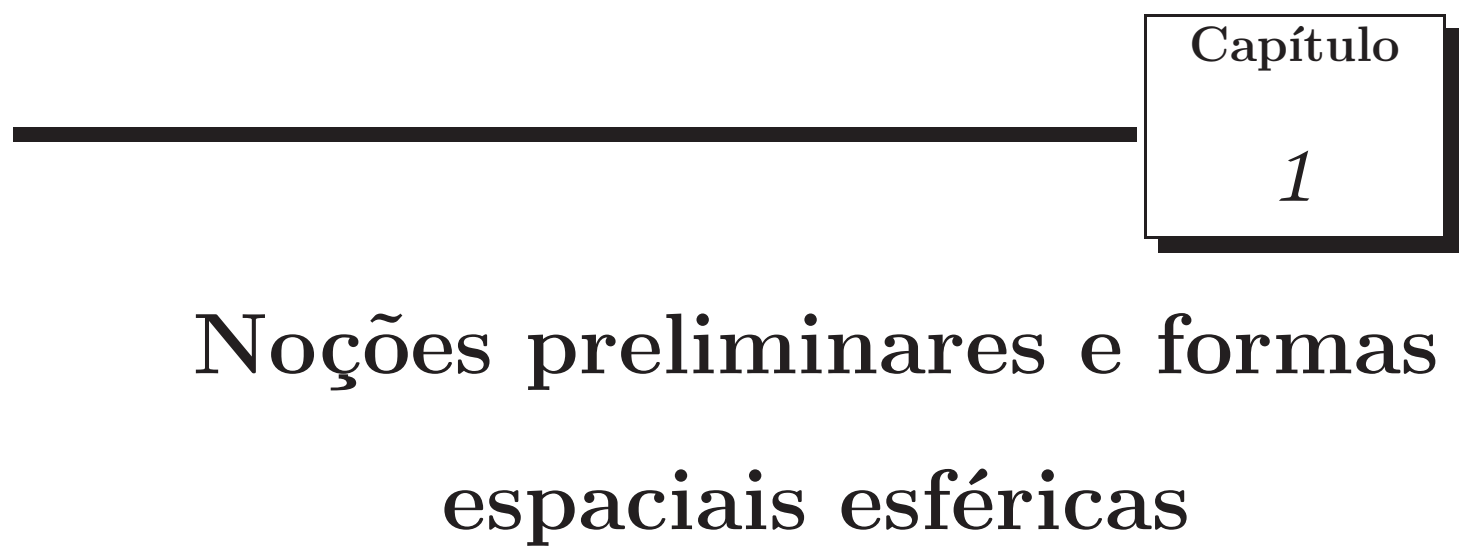

Neste capítulo, apresentamos algumas noções preliminares sobre topologia algébrica e álgebra homológica, as quais serão utilizadas no decorrer da tese. Também são apresentadas algumas definições básicas e alguns resultados que fornecem os possíveis grupos que podem ocorrer na construção das formas espaciais esféricas, cuja referência principal é [28]. Em toda a tese, utilizamos o termo aplicação para nos referirmos a uma função contínua.

\subsection{Ação de grupos e resoluções projetivas e livres}

Definição 1.1.1 Sejam $G$ um grupo e X um espaço topológico não vazio. Uma ação (à esquerda) de $G$ em $X$ é uma aplicação $\phi: G \times X \rightarrow X$ tal que $\phi(g, x)=$ gx satisfazendo, para todo $x$ pertencente a $X$, as condições:

(i) $e x=x \quad(e$ : elemento neutro de $G)$;

(ii) $\left(g_{1} g_{2}\right) x=g_{1}\left(g_{2} x\right)$, para todo $g_{1}$ e $g_{2}$ pertencentes a $G$.

Equivalentemente, podemos escrever esta definição da seguinte forma: uma ação de $G$ em $X$ é um homomorfismo $\varphi: G \rightarrow \mathfrak{B}(X)$ dado por $\varphi(g)=\varphi_{g}$ tal que $\varphi_{g}(x)=g x$, onde $\mathfrak{B}(X)=\{f: X \rightarrow X \mid f$ é um homeomorfismo $\}$ é um grupo com a operação de composição. 
Observação 1.1.2 Um grupo $G$ atuando em um espaço topológico $X$ é denominado G-espaço topológico.

Definição 1.1.3 Dizemos que $X$ é um $G$-espaço topológico livre se a ação de $G$ em $X$ é livre, isto é, $g x=x$, para algum $x$ pertencente a $X$ se, e somente se, $g=e$.

Dizemos que $X$ é um $G$-espaço topológico trivial se a ação de $G$ em X é trivial, isto é, $g x=x$, para todo $x$ pertencente a $X$ e para todo $g$ pertencente a $G$.

Definição 1.1.4 Seja $G$ um grupo. Dizemos que um grupo abeliano livre (ou $\mathbb{Z}$-módulo livre) gerado por todo g pertencente a $G$ é o anel grupo de $G$, denotado por $\mathbb{Z}[G]$ ou simplesmente $\mathbb{Z} G$.

A multiplicação é definida por $\left(\sum m_{i} g_{i}\right)\left(\sum n_{j} g_{j}\right)=\sum m_{i} n_{j}\left(g_{i} g_{j}\right)$.

De agora em diante, tomamos $R=\mathbb{Z} G$, que é um anel associativo e possui unidade 1.e, onde $1 \in \mathbb{Z}$ e $e \in G$ é o elemento neutro de $G$. Por simplicidade, denotamos por 1 a unidade de $R$. Note também que $R$ é comutativo se, e somente se, $G$ é comutativo.

Definição 1.1.5 Um R-módulo $M$ é dito trivial se $g . x=x$ para todo $g \in G$ e $x \in M$.

Definição 1.1.6 Seja $C=\left\{C_{n}, \partial_{n}\right\}_{n \geq 0}\left(\partial_{0}=0\right)$, um complexo de cadeias de $R$-módulos. Considere $\mathbb{Z}$ com a estrutura de R-módulo trivial. Suponha que $\varepsilon: C_{0} \rightarrow \mathbb{Z}$ é um $R$-epimorfismo satisfazendo $\varepsilon \partial_{1}: C_{1} \rightarrow \mathbb{Z}$ é zero. Então, a R-aplicação é é chamada aumentação (sobre $\mathbb{Z})$, e o complexo de cadeias $C$ juntamente com a aumentação $\varepsilon$ é chamado complexo de cadeias aumentado, e denotado por $C \stackrel{\varepsilon}{\rightarrow} \mathbb{Z}$.

Definição 1.1.7 Uma resolução de $\mathbb{Z}$ sobre $R$ é um complexo de cadeias aumentado $C \stackrel{\varepsilon}{\rightarrow} \mathbb{Z}$ de $R$-módulos que é acíclico, ou seja,

$$
C: \cdots \longrightarrow C_{n} \stackrel{\partial_{n}}{\longrightarrow} C_{n-1} \stackrel{\partial_{n-1}}{\longrightarrow} \cdots \longrightarrow C_{1} \stackrel{\partial_{1}}{\longrightarrow} C_{0} \stackrel{\varepsilon}{\longrightarrow} \mathbb{Z} \longrightarrow 0
$$

é exata.

Se cada $C_{n}$ é projetivo (resp. livre), dizemos que a resolução é projetiva (resp. livre).

Proposição 1.1 .8 ([14], III.1.1) Dado um $R$-módulo $X$ sempre podemos construir uma $R$-resolução livre de $X$. 


\subsection{Produto direto e semidireto}

Definição 1.2.1 Se $H$ e $K$ são grupos, então o seu produto direto , denotado por $H \times K$, é o grupo formado por todos os elementos ordenados $(h, k)$, onde $h$ e $k$ pertencem a $H e$ $K$, respectivamente, com a seguinte operação: $(h, k) .\left(h^{\prime}, k^{\prime}\right)=\left(h h^{\prime}, k k^{\prime}\right)$.

Teorema 1.2.2 ([22], Teorema 2.29) Seja $G$ um grupo com subgrupos normais $H$ e $K$. Se $H K=G$ e $H \cap K=1$, então $G \simeq H \times K$.

Definição 1.2.3 Seja K um subgrupo (não necessariamente normal) de G. Um subgrupo $Q$ de $G$ é um complemento de $K$ em $G$ se $K \cap Q=1$ e $K Q=G$.

Definição 1.2.4 Um grupo $G$ é o produto semidireto de $K$ por $Q$, denotado por $G=K \rtimes Q$ se $K$ é um subgrupo normal de $G$ e tem complemento $Q_{1} \simeq Q$.

Observação 1.2.5 Se $Q_{1}$ é um subgrupo normal, então pelo teorema anterior segue que, $G$ é o produto direto $K \times Q_{1}$.

Lema 1.2.6 ([22], Lema 7.21) Se $G$ é um produto semidireto de $K$ por $Q$, então existe um homomorfismo $\varphi: Q \rightarrow$ Aut $(K)$, definido por

$$
\varphi_{x}(a)=x a x^{-1}
$$

para todo $x$ pertencente a $Q$ e a pertencente a $K$. Além disso, para todo $x$, y e 1 pertencentes a $Q$ e a pertencente a $K$,

$$
\varphi_{1}(a)=a \text { e } \varphi_{x}\left(\varphi_{y}(a)\right)=\varphi_{x y}(a)
$$

Definição 1.2.7 Sejam $Q$ e $K$ grupos, e seja $\varphi: Q \rightarrow$ Aut $(K)$ um homomorfismo. Um produto semidireto $G$ de $K$ por $Q$ realiza $\varphi$ se, para todo $x$ pertencente a $Q$ e a pertencente $a K$,

$$
\varphi_{x}(a)=x a x^{-1}
$$

Definição 1.2.8 Considere os grupos $Q$ e $K$, e um homomorfismo $\varphi: Q \rightarrow$ Aut $(K)$, defina $G=K \rtimes_{\varphi} Q$ como sendo o conjunto de todos os pares ordenados $(a, x) \in K \times Q$ com a operação: $(a, x) \cdot(b, y)=\left(a \varphi_{x}(b), x y\right)$. 
Teorema 1.2.9 ([22], Teorema 7.22) Considere os grupos $Q$ e $K$, e um homomorfismo $\varphi: Q \rightarrow$ Aut $(K)$, então $G=K \rtimes_{\varphi} Q$ é um produto semidireto de $K$ por $Q$ que realiza $\varphi$.

Teorema 1.2.10 ([22], Teorema 7.23) Se G é um produto semidireto de $K$ por $Q$, então existe um homomorfismo $\varphi: Q \rightarrow$ Aut $(K) \operatorname{com} G \simeq K \rtimes_{\varphi} Q$.

\subsection{Representação de grupos finitos}

Definição 1.3.1 Sejam $G$ um grupo e $V$ um espaço vetorial sobre um corpo $\mathbb{F}$. Uma representação de $G$ em $V$ é um homomorfismo $\pi$ de $G$ no grupo das transformações lineares invertiveis de $V$. O homomorfismo $\pi$ é uma representação sobre $\mathbb{F}$ e $V$ é chamado o espaço representação de $\pi$.

Se $V$ é de dimensão finita (e neste caso dizemos que $\pi$ é de dimensão finita), então a dimensão de $V$ é chamada de grau de $\pi$.

A representação $\pi$ é chamada de fiel se é injetora.

Definição 1.3.2 Se $\psi$ é outra representação de $G$ em um espaço vetorial $W$ sobre o mesmo corpo $\mathbb{F}$, então $\pi$ e $\psi$ são equivalentes se existe um isomorfismo $f: V \rightarrow W$ tal que $f \pi(g)=\psi(g) f$, para todo $g$ pertencente a $G$.

Definição 1.3.3 Se $U \subseteq V$ é um subespaço $\pi(G)$-invariante, então $\pi^{\prime}(g): u \mapsto \pi(g) u$ define uma representação $\pi^{\prime}: G \rightarrow U$. Representações $\pi^{\prime}$ desta forma são chamadas subrepresentações de $\pi$. Uma subrepresentação $\pi^{\prime}$ de $U$ é própria se $U$ é um subespaço próprio de $V$, isto é, se $0 \subsetneq U \subsetneq V$. A representação $\pi$ é chamada irredutível se não possui subrepresentações próprias.

Definição 1.3.4 Se $\left\{\pi_{i}\right\}$ são representações em espaços vetoriais $\left\{V_{i}\right\}$ sobre $\mathbb{F}$, então a soma direta $\oplus \pi_{i}$ é a representação na soma direta $\oplus V_{i}$ dada por

$$
\left(\oplus \pi_{i}\right)(g): \oplus v_{i} \mapsto \oplus \pi_{i}(g) v_{i}, v_{i} \text { pertencente } a V_{i}
$$

A representação $\pi$ é totalmente irredutivel se é equivalente a uma soma direta de representações irredutiveis. 
Definição 1.3.5 Se $\pi$ e $\psi$ são representações dos grupos $A$ e $B$ nos espaços $V$ e $W$, então o produto tensorial $\pi \otimes \psi$ é a representação de $A \times B$ em $V \otimes W$ dada por

$$
(\pi \otimes \psi)(a, b): v \otimes w \mapsto \pi(a)(v) \otimes \psi(b)(w)
$$

Se $\pi(a)$ e $\psi(b)$ possuem autovalores $\left\{\lambda_{i}\right\}$ e $\left\{\mu_{k}\right\}$, respectivamente, então $\left\{\lambda_{i} \mu_{k}\right\}$ são autovalores de $(\pi \otimes \psi)(a, b)$.

Seja $\sigma$ uma representação irredutível de $G$ em um espaço vetorial de dimensão finita $V$ sobre $\mathbb{K}$. A extensão escalar $V_{\mathbb{F}}=V \otimes_{\mathbb{K}} \mathbb{F}$, com $\mathbb{K} \subset \mathbb{F}$, é um espaço vetorial de mesma dimensão sobre $\mathbb{F}$ que pode ser criado escolhendo uma base $\left\{v_{i}\right\}$ de $V$ sobre $\mathbb{K}$ e definindo $V_{\mathbb{F}}=\left\{\oplus a_{i} v_{i}, a_{i} \in \mathbb{F}\right\}$. Agora $\sigma(G)$ é também um conjunto de transformações $\mathbb{F}$-lineares de $V_{\mathbb{F}}$, assim $\sigma$ nos dá uma representação $\sigma_{\mathbb{F}}$ de $G$ em $V_{\mathbb{F}}$. $\sigma$ é chamada absolutamente irredutível se $\sigma_{\mathbb{F}}$ é irredutível.

Consideremos agora um grupo finito $G$ de ordem $N$ e representações de $G$ sobre o corpo dos números reais $\mathbb{R}$ ou sobre o corpo dos números complexos $\mathbb{C}$. Uma representação sobre $\mathbb{R}$ (resp. sobre $\mathbb{C}$ ) é chamada representação real (resp. representação complexa). Se uma representação complexa $\pi$ é equivalente a $\sigma_{\mathbb{C}}$ para alguma representação real $\sigma$, então cometeremos um abuso de linguagem e diremos que $\pi$ é equivalente a uma representação real.

As isometrias lineares $f: V \rightarrow V$ formam um grupo, chamado grupo ortogonal de $V$ no caso real e grupo unitário de $V$ no caso complexo. Se $V$ é real (resp. complexo), então uma representação de $G$ em $V$ é chamada representação ortogonal (resp. representação unitária) se sua imagem está contida no grupo ortogonal (resp. unitário) de $V$. Se $V$ e $W$ são reais (resp. complexos) e se $\sigma$ e $\tau$ são representações de $G$ em $V$ e $W$, então $\sigma$ e $\tau$ são ortogonalmente equivalentes (resp. unitariamente equivalentes) se existe uma isometria linear $f: V \rightarrow W$ tal que $f \sigma(g)=\tau(g) f$, para todo $g$ pertencente a $G$, e neste caso, é claro, $\sigma$ e $\tau$ são equivalentes.

Lema 1.3.6 ([28], Lema 4.7.1) Toda representação real (resp. complexa) de G é equivalente a uma representação ortogonal (resp. unitária). Duas representações ortogonais (resp. unitárias) de G são equivalentes se, e somente se, são ortogonalmente (resp. 
unitariamente) equivalentes. Se $\sigma$ e $\tau$ são representações ortogonais (resp. unitárias) fiel de $G$ em $V$ e $W$, então existe uma isometria linear $f: V \rightarrow W$ tal que $\tau(G)=f \sigma(G) f^{-1}$ se, e somente se, existe um automorfismo $\alpha$ de $G$ tal que $\tau \alpha$ é equivalente a $\sigma$.

Lema 1.3.7 ([28], Lema 4.7.2) Seja $\pi$ uma representação complexa de $G$. Se $\left\{v_{i}\right\}$ é uma base do espaço representação $V$ e se $\pi(g)$ tem matriz $\left(\pi(g)_{i j}\right)$ relativa a $\left\{v_{i}\right\}$, então seja $\bar{\pi}(g)$ a transformação linear de $V$ cuja matriz relativa a $\left\{v_{i}\right\}$ é a matriz complexa conjugada $\left(\overline{\pi(g)_{i j}}\right)$ de $\left(\pi(g)_{i j}\right)$. Dessa forma, $\bar{\pi}$ é uma representação de $G$ em $V$ que é equivalente à dual $\pi^{*}$ de $\pi$. A representação $\bar{\pi}$ é chamada de conjugada de $\pi$.

Definição 1.3.8 (Grupo livre de ponto fixo) Se $\pi$ é uma representação de um grupo $G$ e se $1 \neq g \in G$ implica que $\pi(g)$ não possui +1 como autovalor, então $\pi$ é livre de ponto fixo. Um grupo livre de ponto fixo é um grupo finito abstrato que possui uma representação livre de ponto fixo.

Proposição 1.3.9 Se G é um grupo livre de ponto fixo que atua na esfera $S^{n}$ de dimensão par então $G=\mathbb{Z}_{2}$.

Demonstração. Suponha que $\varphi: G \times S^{n} \rightarrow S^{n}$ seja uma ação livre de $G$ em $S^{n}$, com $n$ par. Seja $e \neq g \in G$, com $e$ o elemento neutro de $G$, e definimos $L_{g}: S^{n} \rightarrow S^{n}$ por $L_{g}(x)=\varphi(g, x)$. Desde que $L_{g}(x) \neq x$, para todo $x \in S^{n}$, então $L_{g} \sim a$, onde $a: S^{n} \rightarrow S^{n}$ é a aplicação antipodal. Portanto, $\operatorname{deg}\left(L_{g}\right)=\operatorname{deg}(a)=(-1)^{n+1}$. Do mesmo modo, para outro elemento $e \neq h \in G, \operatorname{deg}\left(L_{h}\right)=(-1)^{n+1}$.

Suponha que $g h \neq e$ e assim, $\operatorname{deg}\left(L_{g h}\right)=(-1)^{n+1}$. Por outro lado, $\operatorname{deg}\left(L_{g h}\right)=$ $=\operatorname{deg}\left(L_{g} L_{h}\right)=\operatorname{deg}\left(L_{g}\right) \operatorname{deg}\left(L_{h}\right)=(-1)^{2(n+1)}$. Logo, $2(n+1) \equiv(n+1) \bmod 2$ e assim, $n$ deve ser ímpar, contradizendo a hipótese. Portanto, $g h=e$ para quaisquer $g, h \in G-\{e\}$, ou seja, $G=\mathbb{Z}_{2}$.

Observação 1.3.10 Se $\pi$ é equivalente a uma representação livre de ponto fixo, então $\pi$ é livre de ponto fixo. Se $\pi$ é livre de ponto fixo, então $\pi$ é fiel e é uma soma direta de representações irredutiveis livres de ponto fixo. Se $\left\{\pi_{i}\right\}$ são livres de ponto fixo, então $\oplus \pi_{i}$ é livre de ponto fixo. Se $\pi$ é uma representação sobre um subcorpo de $\mathbb{F}$, então $\pi$ e livre de ponto fixo se, e somente se, $\pi_{\mathbb{F}}$ é livre de ponto fixo. 
Teorema 1.3.11 ([28], Teorema 5.1.2) Dados um grupo finito $G$ e representações ortogonais irredutiveis livres de ponto fixo $\left\{\sigma_{1}, \ldots, \sigma_{r}\right\}$ de $G$, associamos a variedade Riemanniana conexa completa $S^{n-1} /\left(\sigma_{1} \oplus \cdots \oplus \sigma_{r}\right)(G), n=\sum_{i} \operatorname{deg}\left(\sigma_{i}\right)$, de dimensão $n-1$ e curvatura constante $K>0$. Toda variedade Riemanniana conexa completa de curvatura constante positiva é isométrica a uma variedade obtida deste modo e, é chamada de forma espacial esférica. $\left(G,\left\{\sigma_{1}, \ldots, \sigma_{r}\right\}\right)$ e $\left(G,\left\{\tau_{1}, \ldots, \tau_{s}\right\}\right)$ originam variedades isométricas se, e somente se, $r=s$ e existe uma permutação $k \rightarrow i_{k}$ de $\{1, \ldots, r\}$ e um automorfismo $\alpha$ de $G$ tal que $\tau_{k} \alpha$ é equivalente a $\sigma_{i_{k}}$.

O teorema anterior sintetiza o trabalho de G. Vincent [27] e leva à classificação das variedades Riemannianas completas conexas de curvatura constante positiva. Tal problema ficou conhecido como Problema das Formas Espaciais Esféricas de Clifford-Klein. A abordagem utilizada foi a seguinte:

1. encontrar condições necessárias para que um grupo abstrato finito aja nas esferas livre de ponto fixo;

2. classificar os grupos abstratos finitos que satisfazem tais condições, obtendo uma família $\left\{G_{\lambda}\right\}_{\lambda \in \Lambda}$ de grupos;

3. determinar as classes de equivalência de representações complexas irredutíveis livres de ponto fixo de cada $G_{\lambda}$ e aplicar o Lema 1.3.6, visto anteriormente, e o Teorema 4.7.3 de [28], obtendo as classes de equivalência de representações ortogonais irredutíveis livres de ponto fixo;

4. determinar o grupo de automorfismos de cada $G_{\lambda}$ e decidir quando duas representações ortogonais irredutíveis livres de ponto fixo são equivalentes, módulo um automorfismo.

As condições necessárias que devemos encontrar para um grupo abstrato finito agir livre de ponto fixo, dependem de alguns fatos envolvendo a teoria de $p$-grupos, os quais apresentaremos a seguir.

Definição 1.3.12 Seja $G$ um grupo de ordem $N$ e p um número primo, então um p-grupo é um grupo no qual a ordem de todo elemento é uma potência de p. 
Pela definição anterior, se $H$ é um subgrupo de $G$ de ordem $p^{\alpha}, p^{\alpha}$ divide a ordem de $G$ que é igual a $N$, então $H$ é um $p$-subgrupo de $G$. Além disso, se $p^{\alpha}$ é a maior potência de $p$ que divide $N$, então $H$ é chamado de $p$-subgrupo de Sylow de $G$.

Definição 1.3.13 Seja $G$ um grupo finito e $p$, q primos. Se todo subgrupo de ordem $p q$ em $G$ é cíclico, dizemos que $G$ satisfaz a pq-condição. Se para um p primo todo subgrupo de ordem $p^{2}$ em $G$ é cíclico, dizemos que $G$ satisfaz a $p^{2}$-condição.

O teorema a seguir nos dá condições necessárias para que um grupo seja livre de ponto fixo. O caso $p=q$ é devido a Burnside [5] e o caso geral, devido a Zassenhaus [29].

Teorema 1.3.14 ([28], Teorema 5.3.1) Seja $G$ um grupo finito que admite uma representação livre de ponto fixo sobre um corpo $\mathbb{F}$. Se $H$ é um subgrupo de ordem pq em $G$, com p e q primos, então $H$ é cíclico.

Pelo teorema anterior e [28, Teorema 5.3.2], temos que os grupos livres de ponto fixo se dividem em duas classes. A primeira classe consiste de todos os grupo finitos nos quais todo subgrupo de Sylow é cíclico; a segunda classe consiste de todos os grupos finitos nos quais os subgrupos de Sylow ímpar são cíclicos e os 2-subgrupos de Sylow são os quatérnios generalizados $\mathbb{Q}_{2^{a}}, a \geq 3$. Os grupos da primeira classe estão completamente caracterizados no teorema a seguir.

Teorema 1.3.15 ([28], Teorema 5.4.1 (Burnside)) Seja $G$ um grupo finito de ordem $N$ no qual todo subgrupo de Sylow é cíclico. Então $G$ é gerado por $\{A, B\}$ com relações

$$
A^{m}=B^{n}=1, \quad B A B^{-1}=A^{r}, \quad N=m n, \quad \operatorname{mdc}((r-1) n, m)=1, \quad r^{n} \equiv 1 \bmod m .
$$

O grupo comutador $G^{\prime}$ é gerado por $\{A\}$ e o grupo quociente $G / G^{\prime}$ é gerado pela classe $\left\{B G^{\prime}\right\}$. Seja d a ordem de $r$ no grupo multiplicativo de resíduos módulo $m$ dos inteiros primos com $m$. Então d divide $n$ e $G$ satisfaz todas as pq-condições se, e somente se, $n / d$ é divisível por todo divisor primo de d.

Reciprocamente, qualquer grupo dado por (1.3.1) tem ordem $N$ e todo subgrupo de Sylow é cíclico. Também são equivalentes: (1) $G$ é cíclico; (2) $A=1$; (3) $r=1$; (4) $d=1$. 
O teorema a seguir nos dá representações de grupos finitos nos quais todo subgrupo de Sylow é cíclico.

Teorema 1.3.16 ([28], Teorema 5.5.6) Seja $G$ um grupo de ordem $N$ que tem geradores $\{A, B\}$ com relações $A^{m}=B^{n}=1, B A B^{-1}=A^{r}, N=m n=m n^{\prime} d$, onde $d$ é a ordem de $r$ em $K_{m}$ (grupo multiplicativo de resíduos módulo $m$ dos inteiros primos com $m)$ e $m d c((r-1) n, m)=1$. Além disso, $G$ satisfaz todas as pq-condições e tem todo subgrupo de Sylow cíclico. Dados $k, l \in \mathbb{Z} \operatorname{com} m d c(k, m)=1=m d c(l, n)$, definimos

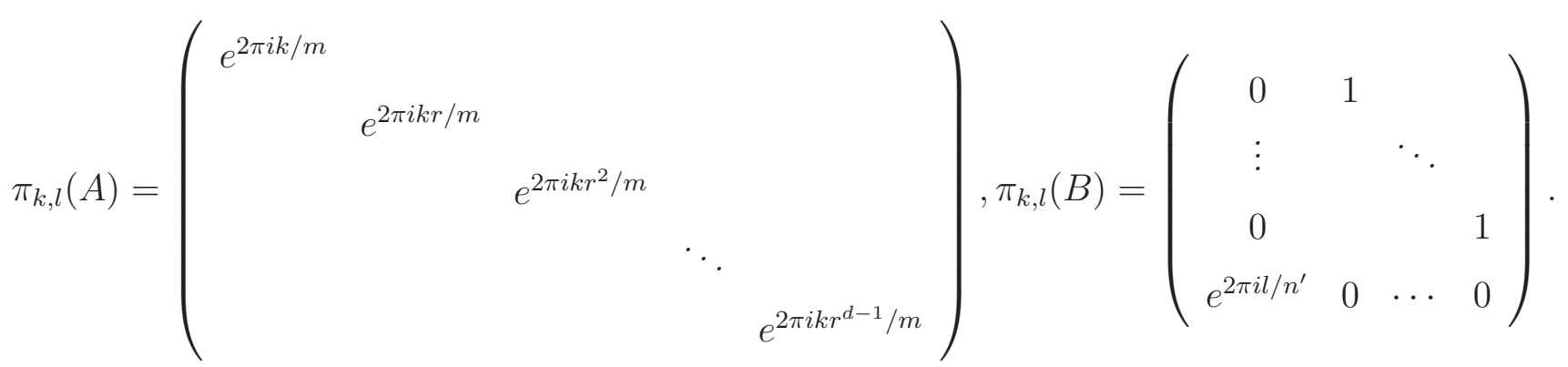

$\operatorname{Dados} s, t, u \in \mathbb{Z}$ com $\operatorname{mdc}(s, m)=1=\operatorname{mdc}(t, n)$ e $t \equiv 1(\bmod d)$, definimos

$$
\psi_{s, t, u}(A)=A^{s} \quad \text { e } \quad \psi_{s, t, u}(B)=B^{t} A^{u}
$$

Então:

1. as representações complexas irredutiveis fiel de $G$ são exatamente as $\pi_{k, l}$ de grau d e livres de ponto fixo;

2. os automorfismos de $G$ são exatamente os $\psi_{s, t, u}$;

3. $\pi_{k, l} \psi_{s, t, u}$ é equivalente $a \pi_{s k, t l} e \pi_{a, b}$ é equivalente $a \pi_{a^{\prime}, b^{\prime}}$ se, e somente se, $b^{\prime} \equiv b\left(\bmod n^{\prime}\right)$ e $a^{\prime} \equiv \operatorname{ar}^{c}(\bmod m)$, para algum inteiro $c$ com $0 \leq c<d ;$

4. se $G$ é cíclico então $\pi_{k, l}$ é equivalente a sua conjugada se, e somente se, $N \leq 2$, e neste caso $\pi_{k, l}$ é equivalente a uma representação real. Se G não é cíclico, então $\pi_{k, l}$ não é equivalente a uma representação real e $\pi_{k, l}$ é equivalente a sua conjugada se, e somente se, $n^{\prime}=2$.

O teorema anterior nos permite descrever as representações reais livres de ponto fixo do grupo $G$. 
No teorema a seguir, $\phi$ denota a função de Eulel1 1 e todas as representações são complexas de dimensão finita.

Teorema 1.3.17 ([28], Teorema 5.5.10) Seja $G$ um grupo de ordem $N$ que satisfaz todas as pq-condições e tem todo subgrupo de Sylow cíclico, como no Teorema 1.3.16 acima. Suponha que $G$ não é cíclico de ordem 1 ou 2. Seja a matriz de rotação

$$
R(\theta)=\left(\begin{array}{cc}
\cos \theta & \operatorname{sen} \theta \\
-\operatorname{sen} \theta & \cos \theta
\end{array}\right)
$$

Dados $k, l \in \mathbb{Z} \operatorname{com} m d c(k, m)=1=m d c(l, n)$, seja $\widehat{\pi}_{k, l}$ a representação real de $G$ de grau $2 d$ definida (em blocos $2 \times 2$ ) por

$$
\widehat{\pi}_{k, l}(A)=\left(\begin{array}{cccc}
R(k / m) & & & \\
& R(k r / m) & & \\
& & \ddots & \\
& & & R\left(k r^{d-1} / m\right)
\end{array}\right), \widehat{\pi}_{k, l}(B)=\left(\begin{array}{cccc}
0 & 1 & \\
\vdots & & \ddots & \\
0 & & & 1 \\
R\left(l / n^{\prime}\right) & 0 & \cdots & 0
\end{array}\right) .
$$

Então uma representação real de $G$ é livre de ponto fixo se, e somente se, é equivalente a uma soma de representações $\widehat{\pi}_{k, l}$. Cada $\widehat{\pi}_{k, l}$ é equivalente a $\widehat{\pi}_{k^{\prime}, l^{\prime}}$ se, e somente se, existem números $e= \pm 1$ e $c \in\{0,1, \ldots, d-1\}$ tais que $k^{\prime} \equiv e k r^{c}(\bmod m)$ e $l^{\prime} \equiv$ el $\left(\bmod n^{\prime}\right)$. Existem exatamente $\phi(N) / d^{2}$ ou exatamente $\phi(N) / 2 d^{2}$ representações $\widehat{\pi}_{k, l}$ não equivalentes, para $n^{\prime}=2$ ou $n^{\prime} \neq 2$, respectivamente.

O lema abaixo merece destaque, pois descreve as representações para um grupo em particular que não se encaixa nos teoremas anteriores, já que não possui todo subgrupo de Sylow cíclico. Mais precisamente, o 2-subgrupo de Sylow do grupo dos quatérnios generalizados de ordem $2^{a}, \mathbb{Q}_{2^{a}}$, é o próprio grupo $\mathbb{Q}_{2^{a}}$, que claramente não é cíclico.

Lema 1.3.18 ([28], Lema 5.6.2) Seja $\mathbb{Q}_{2^{a}}(a \geq 3)$ o grupo dos quatérnios generalizados de ordem $2^{a}$, gerado por $\{A, B\}$ com relações

$$
A^{2^{a-1}}=1, \quad B^{2}=A^{2^{a-2}}, \quad B A B^{-1}=A^{-1} .
$$

\footnotetext{
${ }^{1} \phi(l)$ é a ordem do grupo multiplicativo $K_{l}$ de resíduos módulo $l$ dos inteiros primos com $l$. Assim, $\phi(l)$ é o número de geradores do grupo cíclico $\mathcal{C}_{l}$ de ordem $l$
} 
Se $k$ é um dos $\phi\left(2^{a-2}\right)=2^{a-3}$ números $\left\{1,3,5, \ldots, 2^{a-2}-1\right\}$, definimos a representação $\alpha_{k}$ de $\mathbb{Q}_{2^{a}}$ por

$$
\alpha_{k}(A)=\left(\begin{array}{cc}
e^{2 \pi i k / 2^{a-1}} & 0 \\
0 & e^{-2 \pi i k / 2^{a-1}}
\end{array}\right), \alpha_{k}(B)=\left(\begin{array}{cc}
0 & 1 \\
-1 & 0
\end{array}\right)
$$

Então as seguintes condições são equivalentes:

1. $\alpha$ é uma representação complexa irredutivel fiel de $\mathbb{Q}_{2^{a}}$;

2. $\alpha$ é uma representação complexa irredutíel livre de ponto fixo de $\mathbb{Q}_{2^{a}}$;

3. $\alpha$ é equivalente a alguma $\alpha_{k}$.

Além disso, as $\alpha_{k}$ são mutuamente não equivalentes, cada uma é equivalente a sua conjugada e nenhuma é equivalente a uma representação real.

Os Teoremas 1.3 .11 e 1.3 .17 fornecem uma classificação completa das variedades Riemannianas conexas completas $M$ de curvatura constante positiva, nas quais o grupo fundamental $G$ possui todo subgrupo de Sylow cíclico. Se $G$ é trivial então $M$ é a esfera. Se $G$ é cíclico de ordem 2 então $M$ é o espaço projetivo. Caso contrário, $M$ é isométrica a

$$
S^{2 v d-1} /\left(\widehat{\pi}_{k_{1}, l_{1}} \oplus \cdots \oplus \widehat{\pi}_{k_{v}, l_{v}}\right)(G)
$$

na notação do Teorema 1.3.17, onde $\left\{\left(k_{1}, l_{1}\right), \ldots,\left(k_{v}, l_{v}\right)\right\}$ é determinado por $M$ no sentido de que $M$ é também isométrica a uma variedade $S^{2 v d-1} /\left(\widehat{\pi}_{a_{1}, b_{1}} \oplus \cdots \oplus \widehat{\pi}_{a_{v}, b_{v}}\right)(G)$ se, e somente se, existe uma permutação $j \rightarrow i_{j}$ e existem inteiros $e_{j}, c_{j}, s, t \in \mathbb{Z}$ tais que $e_{j}= \pm 1,0 \leq c_{j}<d, \operatorname{mdc}(s, m)=1=\operatorname{mdc}(t, n), t \equiv 1(\bmod d), k_{i_{j}} \equiv e_{j} s a_{j} r^{c_{j}}(\bmod m) \mathrm{e}$ $l_{i_{j}} \equiv e_{j} t b_{j}\left(\bmod n^{\prime}\right)$.

\subsection{Classificação dos grupos livres de ponto fixo}

Em [28, Capítulo 6] encontramos a classificação dos grupos finitos que possuem representações livres de ponto fixo, divididos em seis tipos. Os quatro primeiros tipos são para grupos solúveis e os outros dois, para não solúveis. Em [28, Capítulo 7] é feita 
a classificação, a menos de automorfismos, das classes de equivalência de representações ortogonais de cada um dos seis tipos de grupos, obtidos anteriormente. Isso soluciona o problema das formas espaciais esféricas de Clifford-Klein.

O teorema a seguir, classifica os grupos solúveis que satisfazem todas as $p^{2}$ ou $p q$-condições, que engloba o grupo de nosso interesse nos próximos capítulos.

Teorema 1.4.1 ([28], Teorema 6.1.11) Seja G um grupo solúvel finito. Então todo subgrupo abeliano de $G$ é cíclico se, e somente se, $G$ é isomorfo a um dos seguintes grupos

\begin{tabular}{|c|c|c|c|c|}
\hline Tipo & Geradores & Relações & Condições & Ordem \\
\hline$I$ & $A, B$ & $\begin{array}{l}A^{m}=B^{n}=1 \\
B A B^{-1}=A^{r}\end{array}$ & $\begin{array}{l}m \geq 1, n \geq 1 \\
r^{n} \equiv 1(\bmod m) \\
m d c(n(r-1), m)=1\end{array}$ & $m n$ \\
\hline II & $A, B, R$ & $\begin{array}{l}\text { Como em } I, \text { e também } \\
R^{2}=B^{n / 2}, R A R^{-1}=A^{l}, \\
R B R^{-1}=B^{k}\end{array}$ & $\begin{array}{l}\text { Como em I, e também } \\
l^{2} \equiv r^{k-1} \equiv 1(\bmod m) \\
n=2^{u} v, u \geq 2 \\
k \equiv-1\left(\bmod 2^{u}\right) \\
k^{2} \equiv 1(\bmod n)\end{array}$ & $2 m n$ \\
\hline III & $A, B, P, Q$ & $\begin{array}{l}\text { Como em } I, \text { e também } \\
P^{4}=1, P^{2}=Q^{2}=(P Q)^{2}, \\
A P=P A, A Q=Q A, \\
B P B^{-1}=Q, B Q B^{-1}=P Q\end{array}$ & $\begin{array}{l}\text { Como em I, e também } \\
n \equiv 1(\bmod 2) \\
n \equiv 0(\bmod 3)\end{array}$ & $8 m n$ \\
\hline$I V$ & $A, B, P, Q, R$ & $\begin{array}{l}\text { Como em III, e também } \\
R^{2}=P^{2}, R P R^{-1}=Q P \\
R Q R^{-1}=Q^{-1} \\
R A R^{-1}=A^{l}, R B R^{-1}=B^{k}\end{array}$ & $\begin{array}{l}\text { Como em III, e também } \\
k^{2} \equiv 1(\bmod n) \\
k \equiv-1(\bmod 3) \\
r^{k-1} \equiv l^{2} \equiv 1(\bmod m)\end{array}$ & $16 m n$ \\
\hline
\end{tabular}

Além disso, as seguintes condições são equivalentes:

1. G possui uma representação complexa livre de ponto fixo;

2. G satisfaz todas as pq-condições 
3. G é do tipo I, II, III ou IV acima, com a seguinte condição adicional: se d é a ordem de $r$ no grupo multiplicativo de resíduos módulo $m$, dos inteiros primos com $m$, então $n / d$ é divisivel por todo divisor primo de d.

Os grupos não solúveis são classificados em dois tipos, cujos detalhes podem ser vistos em [28, Seção 6.3].

Neste trabalho, estaremos interessados em um grupo do Tipo III, da tabela vista no Teorema 1.4.1. o qual será apresentado no próximo capítulo. Dessa forma, os resultados descritos a seguir serão considerados apenas para grupos do Tipo III, mais específicamente para o primeiro dos três casos envolvendo grupos de tal tipo.

Seja $G$ um grupo finito que admite uma representação livre de ponto fixo. Seja $\mathfrak{F}_{\mathbb{C}}(G)$ o conjunto de todas as classes de equivalência de representações complexas irredutíveis livres de ponto fixo de $G$. Descreveremos $\mathfrak{F}_{\mathbb{C}}(G)$, estabelecendo uma notação para seus elementos. Em [28, Teorema 7.2.18] existe uma descrição completa de $\mathfrak{F}_{\mathbb{C}}(G)$, para os outros cinco tipos de grupos.

Tipo III. $G$ é gerado por $\{A, B, P, Q\}$, onde $\{A, B\}$ é um grupo de ordem ímpar do Tipo I, $P^{4}=1, P^{2}=Q^{2}, P Q P^{-1}=Q^{-1}, A P=P A, A Q=Q A, B P B^{-1}=Q$ e $B Q B^{-1}=P Q$.

Caso $1 . n \not 0(\bmod 9)$. Em particular, $d \not 0(\bmod 3)$. Dessa forma, $G=\left\{A, B^{3}\right\} \times$ $\times\left\{B^{n^{\prime \prime}}, P, Q\right\}$, onde $n^{\prime \prime}=n / 3 . \quad\left\{B^{n^{\prime \prime}}, P, Q\right\}$ é o grupo binário tetraedral $P_{24}$, e $\left\{A, B^{3}\right\}$ é um grupo do Tipo I que possui o mesmo inteiro $d$ que $\{A, B\}$. Agora, é imediato pelos Lemas 6.3 .2 e 7.1 .3 de [28] que $\mathfrak{F}_{\mathbb{C}}(G)$ consiste de $\phi\left(m n^{\prime \prime}\right) / d^{2}=\phi(m n) / 2 d^{2}$ representações de grau $2 d$ dadas por

$$
v_{k, l}=\pi_{k, l} \otimes \tau \text {, onde } \pi_{k, l} \in \mathfrak{F}_{\mathbb{C}}\left(\left\{A, B^{3}\right\}\right) \text { e }\{\tau\}=\mathfrak{F}_{\mathbb{C}}\left(P_{24}\right) .
$$

Ação dos automorfismos nas representações. Seja $G$ um grupo finito que admite uma representação complexa livre de ponto fixo. Dado o conjunto $\mathfrak{F}_{\mathbb{C}}(G)$ de todas as classes de equivalência de representações complexas irredutíveis livres de ponto fixo, e os automorfismos de $G$ atuam em $\mathfrak{F}_{\mathbb{C}}(G)$ por composição $\psi: \pi \rightarrow \pi \psi$. Definimos $\mathcal{U}_{\mathbb{C}}(G)$ como sendo o grupo das transformações de $\mathfrak{F}_{\mathbb{C}}(G)$ induzidas por automorfismos de $G$.

A tabela abaixo resume a ação dos automorfismos de $G$ em $\mathfrak{F}_{\mathbb{C}}(G)$ para os grupos que se enquadram no primeiro dos casos do Tipo III. Em [28, Teorema 7.3.21] temos uma descrição 
completa para os outros tipos.

\begin{tabular}{|c|c|c|c|c|}
\hline Tipo & Caso & Elementos de $\mathcal{U}_{\mathbb{C}}(G)$ & ${\operatorname{Ação~em~} \mathfrak{F}_{\mathbb{C}}(G)}^{\text {Condições }}$ \\
\hline \hline III & $n \not 0(\bmod 9)$ & $A_{a, b}$ & $v_{k, l} \rightarrow v_{a k, b l}$ & $\operatorname{mdc}(a, m)=1=m d c(b, n / 3)$ \\
& & & & $b \equiv 1(\bmod d)$ \\
\hline
\end{tabular}

\subsection{Classificação das formas espaciais esféricas}

Os Teoremas 5.1.2, 7.2.18 e 7.3.21 de [28] classificam as variedades Riemannianas conexas completas $M$ de curvatura constante positiva, a menos de isometria global. O Teorema 5.1.2 de [28] é o Teorema 1.3.11] deste capítulo, e os Teoremas 7.2.18 e 7.3.21 de [28] englobam os resultados (particularizados) da Seção 1.4, vista anteriormente.

Seja $G$ o grupo fundamental de $M$. Então $M$ é obtida como segue: se $G$ possui ordem 1 ou 2, então $M$ é isométrica à esfera ou ao espaço projetivo, respectivamente, de dimensões apropriadas.

Se $G$ é do Tipo III com $n \nsucceq 0(\bmod 9)$, então $M$ é isométrica a alguma

$$
S^{4 s d-1} /\left\{\left(\widehat{v}_{k_{1}, l_{1}} \oplus \cdots \oplus \widehat{v}_{k_{s}, l_{s}}\right)(G)\right\}
$$

onde $2\left(\widehat{v}_{k, l}\right)_{\mathbb{C}}=v_{k, l} \oplus \overline{v_{k, l}}$. O conjunto $\left\{\left(k_{i}, l_{i}\right)\right\}$ é determinado a menos de uma transformação $\left(k_{i}, l_{i}\right) \rightarrow\left(x_{i}, y_{i}\right)$ para a qual existe uma permutação $i \rightarrow i^{\prime}$ de $\{1, \ldots, s\}$ e existem inteiros $e_{i}, u_{i}, a$ e $b$ tais que

$$
\begin{gathered}
e_{i}= \pm 1, \quad 0 \leq u_{i}<d, \quad \operatorname{mdc}(a, m)=1=\operatorname{mdc}(b, n / 3), \quad b \equiv 1(\bmod d) \\
x_{i^{\prime}} \equiv e_{i} a k_{i} r^{u_{i}}(\bmod m) \quad \text { e } \quad y_{i^{\prime}} d \equiv e_{i} b l_{i} d(\bmod n / 3)
\end{gathered}
$$

Os demais tipos são descritos em [28, Seção 7.4].

\footnotetext{
${ }^{2}$ Lembramos que uma representação real $\omega$ pode ser vista como uma representação complexa $\omega_{\mathbb{C}}$ e que $\zeta \oplus \bar{\zeta}$ é da forma $\omega_{\mathbb{C}}$ para toda representação complexa $\zeta$
} 


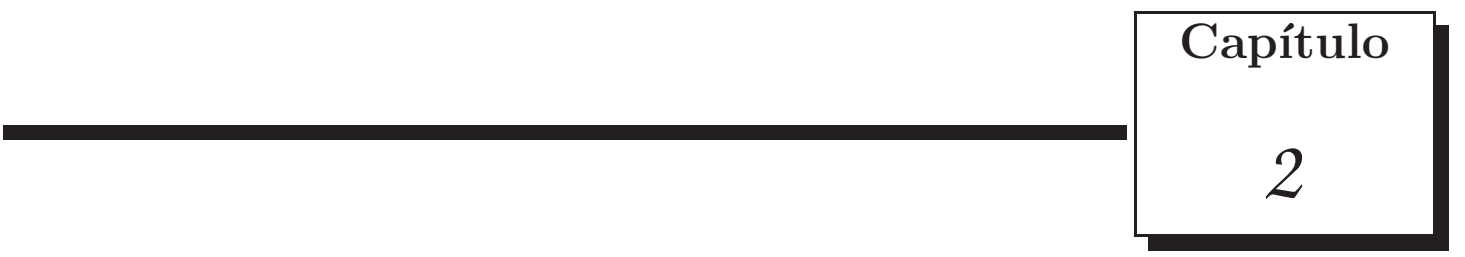

\section{Formas espaciais esféricas tetraedrais}

Neste capítulo, apresentamos o grupo binário tetraedral e suas relações. Estudamos a ação deste grupo nas esferas de dimensão ímpar $\left(S^{4 n-1}, n \geq 1\right)$ e, usamos a geometria para encontrar uma decomposição celular desse espaço, determinando assim, uma região fundamental e um complexo de cadeias coerente com tal ação, afim de lidarmos com o espaço quociente, o qual chamamos de forma espacial esférica tetraedral.

\subsection{Grupo binário tetraedral e algumas notações}

Denotemos por $P_{24}$ o grupo binário tetraedral de ordem 24 , ou seja, $\left|P_{24}\right|=24$, com apresentação [30, Seção 2.2]

$$
P_{24}=\left\langle x, y, z \mid x^{2}=(x y)^{2}=y^{2}, z x z^{-1}=y, z y z^{-1}=x y, x y x^{-1}=y^{-1}, z^{3}=x^{4}=1\right\rangle .
$$

Como de costume, escrevemos $x^{0}=1$, o elemento neutro do grupo.

Analisando a apresentação do grupo, observamos que a ordem do gerador $z$ é igual a três e as ordens dos geradores $x$ e $y$ são iguais a 4 , ou seja, $|z|=3$ e $|x|=4=|y|$.

O grupo binário tetraedral possui a seguinte estrutura de produto semidireto [26, Subseção 5.1]

$$
P_{24} \cong \mathbb{Q}_{8} \rtimes_{\varphi} C_{3}
$$


$\operatorname{com} \varphi: C_{3} \rightarrow \operatorname{Aut}\left(\mathbb{Q}_{8}\right)$ definida por: $\varphi_{z}(x)=z x z^{-1}=y$ e $\varphi_{z}(y)=z y z^{-1}=x y$. Isto é mostrado pela sequência exata curta abaixo, que cinde:

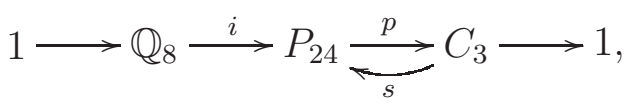

onde $\mathbb{Q}_{8}$ é o subgrupo gerado por $x$ e $y, C_{3}$ é o grupo cíclico com gerador $z, i$ é a inclusão do subgrupo normal gerado por $i(x)=x$ e $i(y)=y, p(z)=z$ (já que $z$ é gerador de $C_{3}$ ), $p(x)=1=p(y)$ e, a aplicação pela qual a sequência cinde é $s(z)=z$.

Notemos que $\left(P_{24}\right)_{a b}=\mathbb{Z}_{3}$.

De fato, temos $\left(P_{24}\right)_{a b}=\langle x, y, z| 2 x=2(x+y)=2 y, z+x-z=y, z+y-z$ $=x+y, x+y-x=-y, 3 z=4 x=0\rangle=\langle x, y, z| 2 x=0=2 y, x=y, x=0, x+y-x$ $=-y, 3 z=0\rangle=\langle z \mid 3 z=0\rangle \approx \mathbb{Z}_{3}$.

Podemos obter algumas relações úteis para o grupo binário tetraedral, são elas:

$$
\begin{array}{ccc}
x^{2}=y^{2} & y x=x^{3} y=x y^{3} & y x^{2}=x^{2} y \\
x z=z x y & z x=y z & z y=x y z \\
y x y=x & x z^{2}=z^{2} y & y z^{2}=z^{2} x y .
\end{array}
$$

Dessa forma, listando os elementos do grupo, temos

$$
P_{24}=\left\{1, x, x^{2}, x^{3}, y, x y, x^{2} y, x^{3} y, z, z x, z x^{2}, z x^{3}, z y, z x y, z x^{2} y, z x^{3} y, z^{2}, z^{2} x, z^{2} x^{2}, z^{2} x^{3},\right.
$$
$\left.z^{2} y, z^{2} x y, z^{2} x^{2} y, z^{2} x^{3} y\right\}$.

Logo, $\left|P_{24}\right|=24$, ou seja, a ordem do grupo binário tetraedral é 24.

Observação 2.1.1 Usualmente, o grupo binário tetraedral é denotado pela letra T entretanto, estamos usando a notação $P_{24}$ devido a uma possível futura generalização do grupo poliedral generalizado.

\subsubsection{Ações livres sobre esferas}

O grupo binário tetraedral $P_{24}$ é um grupo do Tipo III, de acordo com a tabela do Teorema 1.4.1, com $A=1, B=z, P=x, Q=y, n=3, m=1, r=1$ e $d=1$.

De fato, tomando $A=1, B=z, P=x$ e $Q=y$ geradores de $P_{24}$, temos 


$$
\begin{gathered}
P^{4}=x^{4}=1, P^{2}=Q^{2}=(P Q)^{2} \Rightarrow x^{2}=y^{2}=(x y)^{2}, \\
A P=P A \Rightarrow x=x, A Q=Q A \Rightarrow y=y, \\
B P B^{-1}=Q \Rightarrow z x z^{-1}=y, \\
B Q B^{-1}=P Q \Rightarrow z y z^{-1}=x y .
\end{gathered}
$$

Note que todas as igualdades encontradas acima são relações do grupo.

Também deve satisfazer as relações do Tipo I, que são

$$
\begin{gathered}
A^{m}=B^{n}=1 \Rightarrow 1^{m}=z^{n}=1 \Rightarrow n=3 \text { e } m=1 . \\
B A B^{-1}=A^{r} \Rightarrow z \cdot 1 . z^{-1}=1^{r} \Rightarrow r \geq 1 .
\end{gathered}
$$

Verifiquemos agora as condições:

$$
\begin{gathered}
n \equiv 1(\bmod 2) \text { e } n \equiv 0(\bmod 3) \\
m=1 \geq 1, n=3 \geq 1, \text { já que } v \geq 1, \\
m d c(3(r-1), 1)=1, \text { já que } r \geq 1 \text { e } v \geq 1 \mathrm{e}, \\
r^{n}=1(\bmod m) \Rightarrow r^{3} \equiv 1(\bmod 1), \operatorname{logo} r=1 .
\end{gathered}
$$

Agora, pela terceira condição do mesmo teorema citado anteriormente, temos que $d$ é a ordem de $r=1$ em $\mathbb{Z}$, assim $d=1$.

Dessa forma, temos o seguinte resultado:

Lema 2.1.2 ([28], Lema 7.1.3) O grupo binário tetraedral $P_{24}$ possui somente uma representação complexa irredutível e livre de ponto fixo. Se $v>1$, então $P_{8.3^{v}}^{\prime}$ possui exatamente $2.3^{v-1}$ representações complexas irredutíveis e livres de ponto fixo, não equivalentes, as quais são denotadas por $\alpha_{k}, \operatorname{com}(k, 3)=1, k$ determinado módulo $3^{v}$, onde $\alpha_{k}(z)$ possui autovalores $e^{2 \pi i\left(k \pm 3^{v-1}\right) / 3^{v}}$. Em particular, $\alpha_{-k}=\bar{\alpha}_{k}$ e $\alpha_{k}\left(z^{3}\right)$ é a matriz escalar $e^{2 \pi i k / 3^{v-1}} I$.

Todas as representações possuem grau 2.

O lema anterior não deixa explícito quais são as representações matriciais dos geradores $x$ e $y$ do grupo $P_{24}$, apresentando somente a forma matricial do gerador $z$.

Como $P_{24} \cong \mathbb{Q}_{8} \rtimes C_{3}$, onde $\mathbb{Q}_{8}$ é o subgrupo gerado por $x$ e $y$, e $C_{3}$ é o grupo cíclico com gerador $z$, as matrizes dos geradores $x$ e $y$ são as mesmas do Lema 1.3.18, com $a=3$.

Dessa forma, para facilitar as contas, uma reformulação do Lema 2.1.2, a qual utilizaremos no decorrer deste trabalho, é dada a seguir: 
Lema 2.1.3 ([23], Lema 2.15) O grupo binário tetraedral $P_{24}$ possui somente uma representação complexa irredutivel livre de ponto fixo. Esta é a representação padrão

$$
\alpha: P_{24} \longrightarrow U(2 n, \mathbb{C})
$$

obtida do grupo binário tetraedral $P_{24}<S O(3)$ e do recobrimento duplo $U(2 n, \mathbb{C}) \rightarrow S O(3)$. Se $v>1$, então $P_{8.3^{v}}^{\prime}$ possui exatamente $2.3^{v-1}$ representações complexas irredutiveis livres de ponto fixo, duas a duas não equivalentes, $\alpha_{k}$, onde $1 \leq k<3^{v},(k, 3)=1$, dadas por

$$
\alpha_{k}(z)=-\frac{1}{2} e^{\frac{2 \pi i k}{3^{\nu}}}\left(\begin{array}{cc}
1+i & 1+i \\
-1+i & 1-i
\end{array}\right), \quad \alpha_{k}(x)=\left(\begin{array}{cc}
i & 0 \\
0 & -i
\end{array}\right), \quad \alpha_{k}(y)=\left(\begin{array}{cc}
0 & 1 \\
-1 & 0
\end{array}\right)
$$

$A$ representação $\alpha$ de $P_{24}$ também é dada pelas fórmulas acima considerando $v=0$.

Agora, consideremos as ações de $P_{24}$ sobre esferas de dimensão ímpar, ou seja, sobre $S^{4 n-1}, n \geq 1$. Por [28, Seção 7.4] a ação é a soma direta dada pela seguinte representação:

$$
\alpha=\alpha_{1} \oplus \alpha_{2} \oplus \alpha_{3} \oplus \cdots \oplus \alpha_{n}: P_{24} \longrightarrow U(2 n, \mathbb{C}),
$$

onde $\alpha_{j}: P_{24} \rightarrow U(2, \mathbb{C}), j=1, \ldots, n$, é dada por

$$
\alpha_{j}(x)=\left(\begin{array}{cc}
i & 0 \\
0 & -i
\end{array}\right), \quad \alpha_{j}(y)=\left(\begin{array}{cc}
0 & 1 \\
-1 & 0
\end{array}\right) \quad \text { e } \alpha_{j}(z)=-\frac{1}{2}\left(\begin{array}{cc}
1+i & 1+i \\
-1+i & 1-i
\end{array}\right)
$$

Definição 2.1.4 (Formas espaciais esféricas tetraedrais) Seja $P_{24}$ o grupo binário tetraedral de ordem 24. Com a ação de $P_{24}$ sobre $S^{4 n-1}, n \geq 1$, descrita anteriormente, formamos o espaço quociente

$$
\mathscr{P}_{4 n-1}=\frac{S^{4 n-1}}{\alpha\left(P_{24}\right)}
$$

chamado forma espacial esférica tetraedral. 


\subsubsection{Curved join}

Seja $w \in \mathbb{C},|w|=1$, então $w=(\cos \phi, \operatorname{sen} \phi) \in \mathbb{R}^{2}$, para algum $\phi \in[0,2 \pi)$. Dados dois pontos $w_{1}=\left(\cos \phi_{1}, \operatorname{sen} \phi_{1}\right)$ e $w_{2}=\left(\cos \phi_{2}, \operatorname{sen} \phi_{2}\right), \phi_{1}, \phi_{2} \in[0,2 \pi), \operatorname{com}\left(w_{1}, w_{2}\right) \in \mathbb{C} \times \mathbb{C}=$ $\mathbb{R}^{4}$, os vetores $\overrightarrow{w_{1}}=\left(\cos \phi_{1}, \operatorname{sen} \phi_{1}, 0,0\right)$ e $\overrightarrow{w_{2}}=\left(0,0, \cos \phi_{2}, \operatorname{sen} \phi_{2}\right)$ são ortogonais e assim, existe um arco de geodésica unitário unindo $\overrightarrow{w_{1}}$ e $\overrightarrow{w_{2}}$. Denotamos por $w_{1} * w_{2}=\left[w_{1}, w_{2}\right]$, o menor arco de geodésica unindo $\overrightarrow{w_{1}}$ e $\overrightarrow{w_{2}}$, e por $w_{1} * \emptyset$ e $\emptyset * w_{2}$ os pontos $w_{1}$ e $w_{2}$, respectivamente.

Para quaisquer dois subconjuntos $W_{1}$ e $W_{2}$, com $W_{1} \times W_{2} \subset S^{1} \times S^{1} \subset \mathbb{C} \times \mathbb{C}$, definimos o curved join dos dois conjuntos por

$$
W_{1} * W_{2}=\left\{w_{1} * w_{2} \mid w_{1} \in W_{1}, w_{2} \in W_{2}\right\}
$$

Notemos, por exemplo, que $S^{1} * S^{1}=S^{3}$.

Generalizamos tal processo, como segue: identificamos $\mathbb{C}^{m}$ com $\mathbb{R}^{2 m}$, e dada a base ortonormal $\left\{e_{1}, \ldots, e_{2 m}\right\}$ de $\mathbb{R}^{2 m}$, para cada $1 \leq r \neq s \leq 2 m$, denotamos $\Pi_{r, s}$ o plano gerado por $\left\{e_{r}, e_{s}\right\}$. Suponhamos $\Pi_{r_{1}, s_{1}} \cap \Pi_{r_{2}, s_{2}}=\{0\}$. Sejam $W_{1}$ e $W_{2}$ subconjuntos dos círculos unitários de $\Pi_{r_{1}, s_{1}}$ e $\Pi_{r_{2}, s_{2}}$, respectivamente. Então o 'curved join' está bem definido pela equação (2.1.2). Em particular, denotamos por $\Sigma_{l}$ o círculo unitário pertencente ao l-ésimo hiperplano $\underbrace{0 \times \ldots \times 0 \times \mathbb{C}}_{l} \times 0 \times \ldots \times 0$ de $\mathbb{C}^{2 n}$. Denotemos por $\Sigma^{l}=\Sigma_{1} * \Sigma_{2} * \cdots \Sigma_{l}$, com $\Sigma^{0}=\emptyset$. Dessa forma, temos um homomorfismo de 'curved join' iterado

$$
S^{4 n-1}=\Sigma^{2 n}=\Sigma_{1} * \Sigma_{2} * \cdots * \Sigma_{2 n}
$$

É conveniente identificar os vetores da base com seus pontos finais, e usar $e_{i}, i=1,2,3,4$, para denotar os pontos em $S^{4 n-1}$. Dessa forma, por exemplo, a base canônica de $\mathbb{R}^{4}$ em $S^{3}$ é

$$
\begin{array}{ll}
e_{1}=(1,0,0,0)=1 * \emptyset, & e_{2}=(0,1,0,0)=i * \emptyset, \\
e_{3}=(0,0,1,0)=\emptyset * 1, & e_{4}=(0,0,0,1)=\emptyset * i .
\end{array}
$$

Também é usual identificar $S^{3}=S^{1} * S^{1}$. Podemos assim representar as duas meia esferas $S^{1} * S_{ \pm}^{1}$ (onde $S_{ \pm}^{1}$ denota o hemisfério norte/sul da $S^{1}$ ) como na figura abaixo, onde 
o esqueleto da figura é dado pelas linhas de geodésicas unindo os pontos $e_{j}$ (vide [7, Seção 26] para mais detalhes).
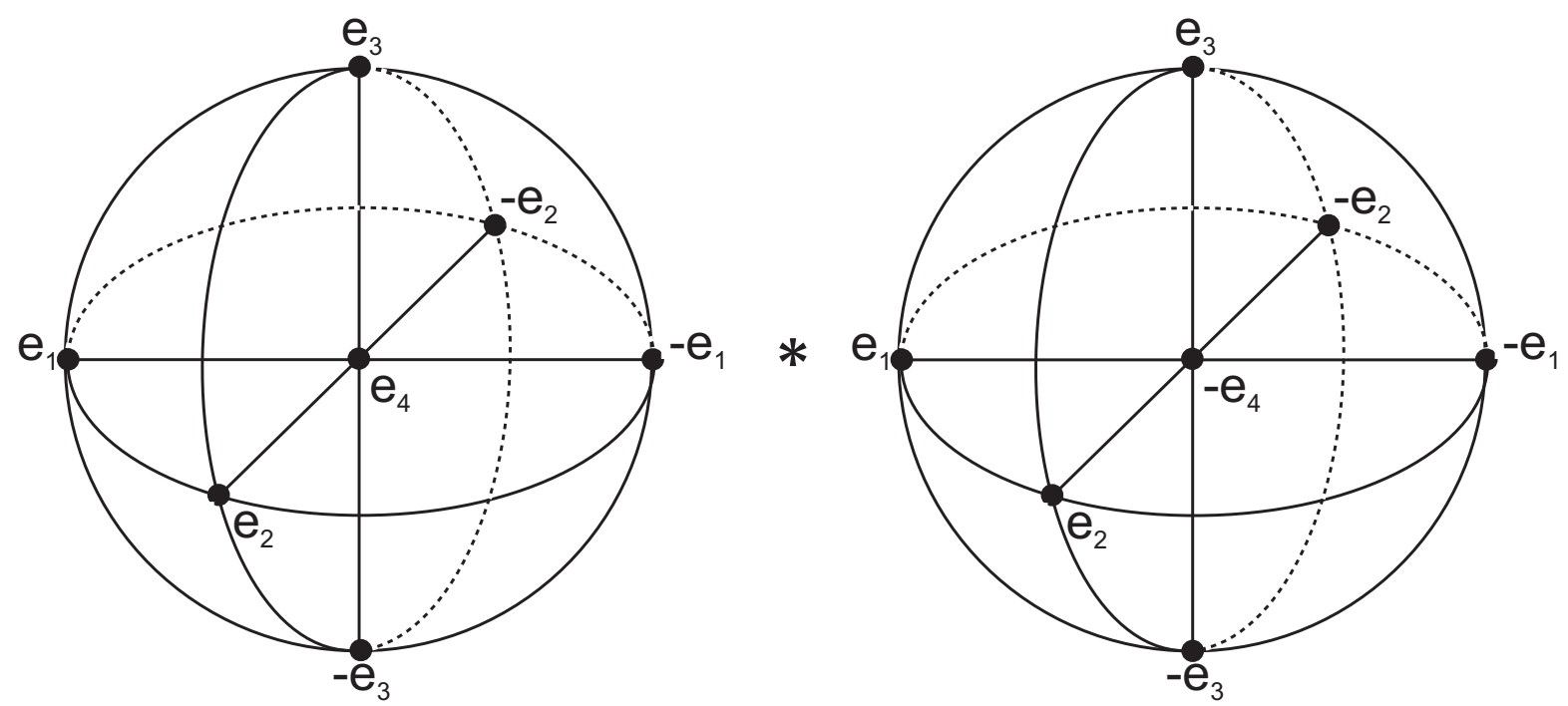

É claro que o 'curved join' é homeomorfo ao 'join' usual:

$$
J(X, Y)=\frac{X \times I \times Y}{(\{x\} \times 0 \times Y) \cup(X \times 1 \times\{y\})} .
$$

Entretanto, o 'join' usual e o 'curved join' não são isométricos. A métrica do 'curved join’ é a métrica da esfera, e os segmentos são segmentos de geodésicas. Em particular, isto é fundamental quando descrevemos a ação natural que aparece na definição das formas espaciais esféricas. Mais precisamente, seja $G$ um grupo finito agindo livremente e ortogonalmente sobre uma esfera $S^{n}$, seja $h$ um inteiro positivo. Então, existe uma ação natural de $G$ sobre $S^{h(n+1)-1}$ definida por

$$
\begin{aligned}
\left(S^{h(n+1)-1} \subset\left(\mathbb{R}^{n+1}\right)^{h}\right) \times G & \rightarrow S^{h(n+1)-1} \subset\left(\mathbb{R}^{n+1}\right)^{h}, \\
\left(\left(x_{1}, \ldots, x_{h}\right), g\right) & \mapsto\left(g x_{1}, \ldots, g x_{h}\right) .
\end{aligned}
$$

Esta ação coincide com a ação

$$
\begin{gathered}
\left(S^{h(n+1)-1}=S^{(h-1)(n+1)-1} * S^{n}\right) \times G \rightarrow S^{h(n+1)-1}=S^{(h-1)(n+1)-1} * S^{n}, \\
((x, t, y), g) \mapsto(g x, t, g y),
\end{gathered}
$$

onde o 'join' é o 'curved join'. 


\section{$2.2 \mathrm{O}$ caso tridimensional}

Nesta seção, apresentamos os dois resultados técnicos mais importantes da tese: na primeira subseção descrevemos a região fundamental (ou domínio fundamental) $\mathfrak{F}_{24,3}$ para a ação de $P_{24}$ via a representação $\alpha$ sobre a esfera tridimensional $S^{3}$; na segunda subseção conseguimos uma decomposição $C W$ de $\mathfrak{F}_{24,3}$, obtendo assim, uma decomposição $C W$ equivariante do espaço $\mathscr{P}_{3}$.

\subsubsection{A região fundamental}

Definição 2.2.1 Seja $G$ um grupo finito agindo sobre um espaço $X$. Uma região (ou dominio) fundamental da ação de $G$ sobre $X$ é um subconjunto fechado e conexo $\mathfrak{F}$ de $X$ tal que $X=\bigcup_{g} g \mathfrak{F}$ e $g \mathfrak{F} \cap g^{\prime} \mathfrak{F}$ possui interior vazio para todo $g \neq g^{\prime} \in G$.

Observação 2.2.2 Denotamos a região fundamental por $\mathfrak{F}_{|G|, 4 n-1}$, onde $|G|$ representa a ordem do grupo e $4 n-1$ representa a dimensão da esfera que estaremos trabalhando.

A primeira diferença importante deste grupo com relação aos quaterniônicos, vistos em [16], e aos metacíclicos, vistos em [10], é que no presente caso quando agimos os 24 elementos do grupo em cada um dos $e_{i}$ 's, $i=1,2,3$, 4 , os pontos resultantes da ação dos 16 elementos do grupo que contém o gerador $z$, apresentam as quatro coordenadas diferentes de zero e dessa forma, não é possível analisarmos se uma dada região é fundamental como foi feito em [16] e [10], onde a região dada era formada pelo 'curved join' de dois arcos de geodésicas, um em $\Pi_{1,2}$ e outro em $\Pi_{3,4}$.

A alternativa encontrada para o grupo $P_{24}$ foi tomar o ponto $a=\left(0,0, \frac{\sqrt{2}}{2},-\frac{\sqrt{2}}{2}\right)$ como ponto base (0-célula), pois agindo os 24 elementos do grupo sobre $a$, os pontos resultantes aparecem em um dos seis planos coordenados, ou seja, em $\Pi_{1,2}, \Pi_{1,3}, \Pi_{1,4}, \Pi_{2,3}, \Pi_{2,4}$ ou $\Pi_{3,4}$, e a partir daí, fica mais fácil encontrarmos uma região fundamental para a forma espacial esférica tetraedral de dimensão três.

Veremos a seguir, a construção de uma região fundamental para a ação do grupo $P_{24}$ sobre $S^{3}$, para isto consideremos a descrição do 'curved join' de $S^{3}=S^{1} * S^{1}$, dada na seção 
anterior.

Calculando a ação de todos os elementos do grupo $P_{24}$, que são $\left\{1, x, x^{2}, x^{3}, y, x y, x^{2} y, x^{3} y\right.$, $\left.z, z x, z x^{2}, z x^{3}, z y, z x y, z x^{2} y, z x^{3} y, z^{2}, z^{2} x, z^{2} x^{2}, z^{2} x^{3}, z^{2} y, z^{2} x y, z^{2} x^{2} y, z^{2} x^{3} y\right\} \quad$ no $\quad$ ponto $a=\left(0,0, \frac{\sqrt{2}}{2},-\frac{\sqrt{2}}{2}\right)$ (usando ação à esquerda), obtemos:

$$
\begin{aligned}
& 1 a=\left(0,0, \frac{\sqrt{2}}{2},-\frac{\sqrt{2}}{2}\right) \quad x a=\left(0,0,-\frac{\sqrt{2}}{2},-\frac{\sqrt{2}}{2}\right) \quad x^{2} a=\left(0,0,-\frac{\sqrt{2}}{2}, \frac{\sqrt{2}}{2}\right) \\
& x^{3} a=\left(0,0, \frac{\sqrt{2}}{2}, \frac{\sqrt{2}}{2}\right) \quad y a=\left(\frac{\sqrt{2}}{2},-\frac{\sqrt{2}}{2}, 0,0\right) \quad x y a=\left(\frac{\sqrt{2}}{2}, \frac{\sqrt{2}}{2}, 0,0\right) \\
& x^{2} y a=\left(-\frac{\sqrt{2}}{2}, \frac{\sqrt{2}}{2}, 0,0\right) \quad x^{3} y a=\left(-\frac{\sqrt{2}}{2},-\frac{\sqrt{2}}{2}, 0,0\right) \quad z a=\left(-\frac{\sqrt{2}}{2}, 0,0, \frac{\sqrt{2}}{2}\right) \\
& z x a=\left(0, \frac{\sqrt{2}}{2}, \frac{\sqrt{2}}{2}, 0\right) \quad z x^{2} a=\left(\frac{\sqrt{2}}{2}, 0,0,-\frac{\sqrt{2}}{2}\right) \quad z x^{3} a=\left(0,-\frac{\sqrt{2}}{2},-\frac{\sqrt{2}}{2}, 0\right) \\
& z y a=\left(-\frac{\sqrt{2}}{2}, 0,0,-\frac{\sqrt{2}}{2}\right) \quad z x y a=\left(0,-\frac{\sqrt{2}}{2}, \frac{\sqrt{2}}{2}, 0\right) \quad z x^{2} y a=\left(\frac{\sqrt{2}}{2}, 0,0, \frac{\sqrt{2}}{2}\right) \\
& z x^{3} y a=\left(0, \frac{\sqrt{2}}{2},-\frac{\sqrt{2}}{2}, 0\right) \quad z^{2} a=\left(\frac{\sqrt{2}}{2}, 0,-\frac{\sqrt{2}}{2}, 0\right) \quad z^{2} x a=\left(0,-\frac{\sqrt{2}}{2}, 0, \frac{\sqrt{2}}{2}\right) \\
& z^{2} x^{2} a=\left(-\frac{\sqrt{2}}{2}, 0, \frac{\sqrt{2}}{2}, 0\right) \quad z^{2} x^{3} a=\left(0, \frac{\sqrt{2}}{2}, 0,-\frac{\sqrt{2}}{2}\right) \quad z^{2} y a=\left(0, \frac{\sqrt{2}}{2}, 0, \frac{\sqrt{2}}{2}\right) \\
& z^{2} x y a=\left(-\frac{\sqrt{2}}{2}, 0,-\frac{\sqrt{2}}{2}, 0\right) \quad z^{2} x^{2} y a=\left(0,-\frac{\sqrt{2}}{2}, 0,-\frac{\sqrt{2}}{2}\right) \quad z^{2} x^{3} y a=\left(\frac{\sqrt{2}}{2}, 0, \frac{\sqrt{2}}{2}, 0\right)
\end{aligned}
$$

Segue abaixo, uma representação tridimensional da esfera $S^{3}$ com todos os pontos resultantes da ação dos elementos do grupo $P_{24}$ em $a=\left(0,0, \frac{\sqrt{2}}{2},-\frac{\sqrt{2}}{2}\right)$. Observemos que os pontos resultantes estarão sempre na metade de cada arco de geodésica dos eixos coordenados, já que estes últimos possuem comprimento $\frac{\pi}{2}$, os pontos resultantes determinam novos arcos de geodésicas de comprimento $\frac{\pi}{4}$.

Lembremos que, os segmentos que possuem $\pm e_{i}, i=1,2,3,4$, como um de seus vértices são, na verdade, arcos de comprimento $\frac{\pi}{2}$. 

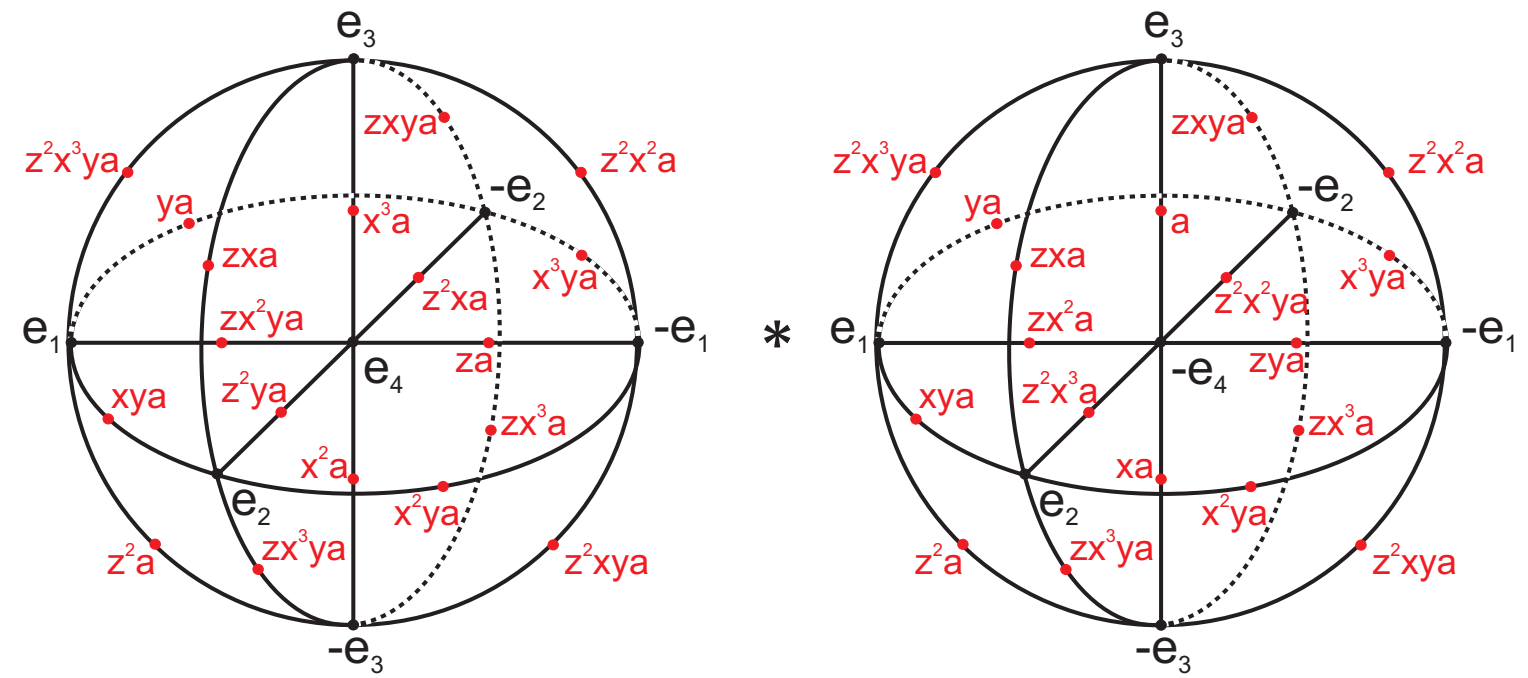

Agora, como queremos encontrar uma região fundamental para a ação de $P_{24}$ sobre $S^{3}$, vale lembrar que devemos verificar se $\mathfrak{F}_{24,3}$ cobre toda a $S^{3}$ e se dados $g, g^{\prime}$ pertencentes a $P_{24} \operatorname{com} g$ e $g^{\prime}$ distintos, a ação de $g$ e a ação de $g^{\prime}$ sobre $\mathfrak{F}_{24,3}$ possuem interiores disjuntos.

Para que a região fundamental cubra toda a $S^{3}$ basta fazermos uma conta simples, envolvendo o volume, ou seja, o volume da região fundamental deve ser igual ao volume da $S^{3}$, o qual denotamos por $V_{S^{3}}$, dividido pela ordem do grupo, pois assim quando aplicamos cada elemento do grupo $P_{24}$, teremos a região fundamental cobrindo toda a $S^{3}$, já que $g \mathfrak{F}_{24,3} \cap g^{\prime} \mathfrak{F}_{24,3}$ possui interior vazio, para todo $g \neq g^{\prime} \in P_{24}$.

$\mathrm{Na}$ primeira figura, estamos considerando a região cujos vértices são os elementos do grupo $P_{24}$ agindo no ponto a, nessa representação estamos usando a descrição de $S^{3}=S^{1} * S^{1}$, introduzida na seção anterior. Na segunda figura, representamos a região segundo o tetraedro no qual ela está inserida, esta será a representação mais utilizada.
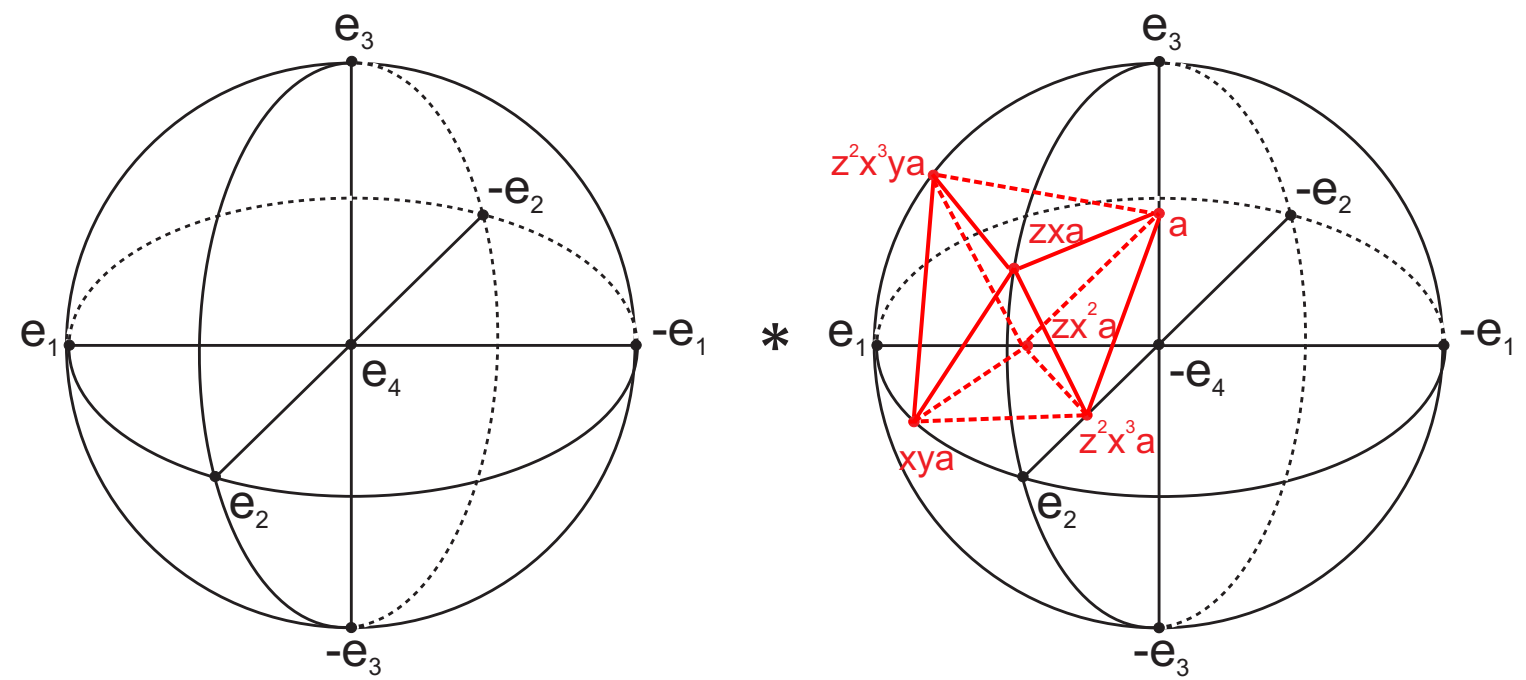


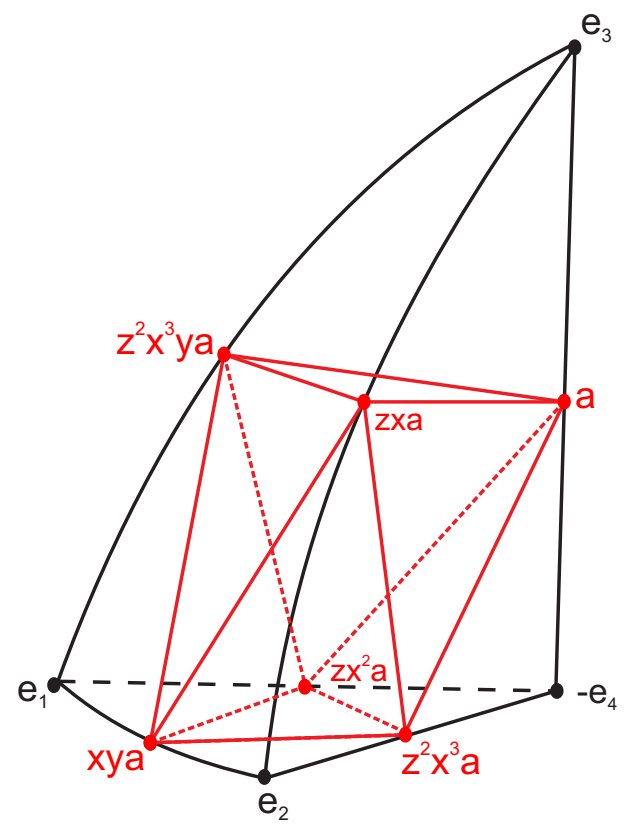

Lema 2.2.3 A imagem da região acima pela ação do grupo $P_{24}$, possui volume igual a $\frac{V_{S^{3}}}{\left|P_{24}\right|}=\frac{V_{S^{3}}}{24}$.

Demonstração. Seja $W$ o volume do tetraedro com vértices $e_{1}, e_{2}, e_{3}$ e $-e_{4}$. Dessa forma, $W=\frac{V_{S^{3}}}{16}$, já que existem 16 tetraedros isométricos a ele cobrindo toda a $S^{3}$.

Consideremos agora, o volume desconhecido do tetraedro com vértices

$$
z x a=\left(0, \frac{\sqrt{2}}{2}, \frac{\sqrt{2}}{2}, 0\right), a=\left(0,0, \frac{\sqrt{2}}{2},-\frac{\sqrt{2}}{2}\right), \quad e_{3}, \quad z^{2} x^{3} y a=\left(\frac{\sqrt{2}}{2}, 0, \frac{\sqrt{2}}{2}, 0\right)
$$

como sendo $A$ e, observemos que as arestas de tal tetraedro possuem comprimento $\frac{\pi}{4}, \frac{\pi}{4}, \frac{\pi}{4}$, $\frac{\pi}{3}, \frac{\pi}{3}$ e $\frac{\pi}{3}$ (vide figura a seguir). Notemos que as arestas dos tetraedros com vértices

$$
\begin{gathered}
x y a=\left(\frac{\sqrt{2}}{2}, \frac{\sqrt{2}}{2}, 0,0\right), z x^{2} a=\left(\frac{\sqrt{2}}{2}, 0,0,-\frac{\sqrt{2}}{2}\right), z^{2} x^{3} y a=\left(\frac{\sqrt{2}}{2}, 0, \frac{\sqrt{2}}{2}, 0\right), e_{1} \\
x y a=\left(\frac{\sqrt{2}}{2}, \frac{\sqrt{2}}{2}, 0,0\right), \quad e_{2}, z^{2} x^{3} a=\left(0, \frac{\sqrt{2}}{2}, 0,-\frac{\sqrt{2}}{2}\right), \quad z x a=\left(0, \frac{\sqrt{2}}{2}, \frac{\sqrt{2}}{2}, 0\right) \\
z^{2} x^{3} a=\left(0, \frac{\sqrt{2}}{2}, 0,-\frac{\sqrt{2}}{2}\right), \quad-e_{4}, \quad a=\left(0,0, \frac{\sqrt{2}}{2},-\frac{\sqrt{2}}{2}\right), \quad z x^{2} a=\left(\frac{\sqrt{2}}{2}, 0,0,-\frac{\sqrt{2}}{2}\right)
\end{gathered}
$$


também possuem comprimento $\frac{\pi}{4}, \frac{\pi}{4}, \frac{\pi}{4}, \frac{\pi}{3}, \frac{\pi}{3}$ e $\frac{\pi}{3}$ (vide figura abaixo), logo cada um deles também terá o volume igual a $A$. Portanto, o volume desses quatro tetraedros é igual a $4 A$.

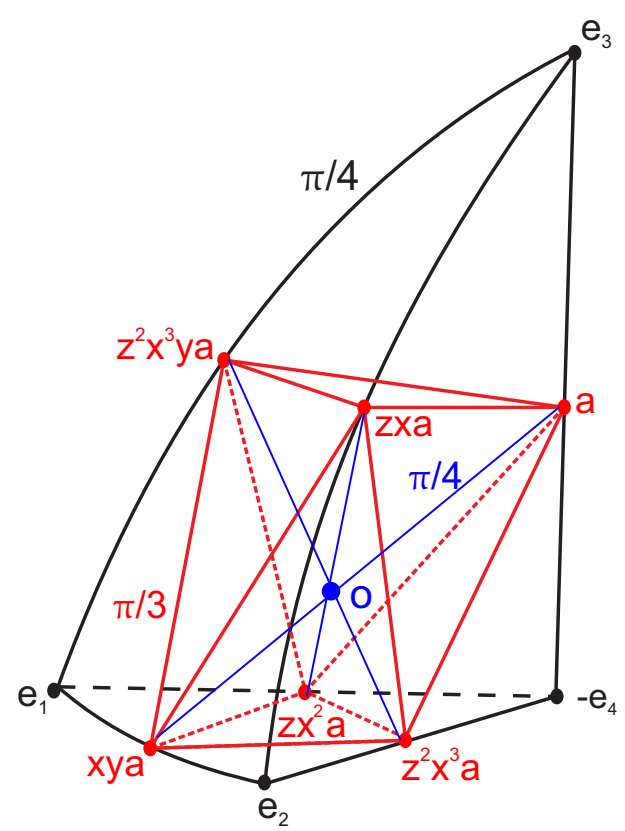

Resta agora encontrar o volume do sólido no interior do tetraedro, com arestas em vermelho e vértices

$$
\begin{aligned}
& z^{2} x^{3} y a=\left(\frac{\sqrt{2}}{2}, 0, \frac{\sqrt{2}}{2}, 0\right), \quad x y a=\left(\frac{\sqrt{2}}{2}, \frac{\sqrt{2}}{2}, 0,0\right), z x a=\left(0, \frac{\sqrt{2}}{2}, \frac{\sqrt{2}}{2}, 0\right), \\
& z^{2} x^{3} a=\left(0, \frac{\sqrt{2}}{2}, 0,-\frac{\sqrt{2}}{2}\right), \quad a=\left(0,0 \frac{\sqrt{2}}{2},-\frac{\sqrt{2}}{2}\right), \quad z x^{2} a=\left(\frac{\sqrt{2}}{2}, 0,0,-\frac{\sqrt{2}}{2}\right),
\end{aligned}
$$

o qual denotaremos por $V_{P}$.

Observemos que, traçando as três diagonais de comprimento $\frac{\pi}{2}$ cada uma, no interior do sólido vermelho, obtemos um ponto de encontro destas, com coordenadas $\left(\frac{1}{2}, \frac{1}{2}, \frac{1}{2},-\frac{1}{2}\right)$, denotaremos esse ponto por $O$. Dessa forma, obtemos oito tetraedros no interior do sólido vermelho, centrados em $O$, cujas arestas de cada um deles possuem comprimento $\frac{\pi}{4}, \frac{\pi}{4}, \frac{\pi}{4}, \frac{\pi}{3}, \frac{\pi}{3}$ e $\frac{\pi}{3}$, ou seja, cada um dos oito tetraedros também possuem volume igual a $A$. Logo, temos

$$
4 A+8 A=W \Longrightarrow 12 A=W \Longrightarrow A=\frac{W}{12}
$$


Portanto,

$$
V_{P}=8 A=8 \cdot \frac{W}{12}=\frac{2}{3} W=\frac{2}{3} \cdot \frac{V_{S^{3}}}{16}=\frac{V_{S^{3}}}{3.8}=\frac{V_{S^{3}}}{24} .
$$

Agora, para que a suposta região seja uma região fundamental para a ação do grupo $P_{24}$, resta mostrar que dados $g, g^{\prime}$ pertencentes a $P_{24}$, com $g$ e $g^{\prime}$ distintos, as ações de $g$ e de $g^{\prime}$ sobre a suposta região fundamental possuem interiores (os quais denotaremos por Int) disjuntos.

Vimos, na equação (2.1.1), que o grupo $P_{24} \simeq \mathbb{Q}_{8} \rtimes_{\varphi} C_{3}$. Como pode ser visto em [16], a região fundamental da ação do grupo $\mathbb{Q}_{8}$ sobre $S^{3}$ (denotada por $\mathfrak{F}_{8,3}$ ) é dada pelo seguinte 'curved join':

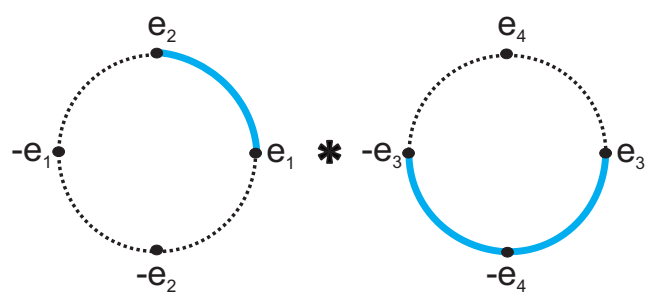

Denotemos por $A_{1}$ o arco de $\Sigma_{1}$ com comprimento $\frac{\pi}{2}$ começando em $e_{1}$ no sentido positivo (ou seja, no sentido anti-horário), e por $A_{2}$ o arco de $\Sigma_{2}$ com comprimento $\pi$ começando em $e_{3}$, no sentido negativo (ou seja, no sentido horário).

Agora, vamos descrever as ações dos geradores $x$ e $y$ do grupo na representação $\alpha$, descritas no Lema 2.1.3. Sejam $\beta_{j}=\left[z_{j}, w_{j}\right]$ um arco de $\Sigma_{j}$ e $R(\theta)$ a rotação do ângulo $\theta$, então:

$$
\begin{gathered}
\alpha(x)\left(\beta_{1} * \beta_{2}\right)=R\left(\frac{\pi}{2}\right)\left(\beta_{1}\right) * R\left(-\frac{\pi}{2}\right)\left(\beta_{2}\right) \\
\alpha(y)\left(\beta_{1} * \beta_{2}\right)=R(\pi)\left(\beta_{2}\right) * \beta_{1} .
\end{gathered}
$$

Observação 2.2.4 $\alpha\left(x^{\gamma}\right)\left(\beta_{1} * \beta_{2}\right)=R\left(\gamma \frac{\pi}{2}\right)\left(\beta_{1}\right) * R\left(-\gamma \frac{\pi}{2}\right)\left(\beta_{2}\right)$.

Uma vez que, a região $\mathfrak{F}_{24,3}$ está no interior da região $\mathfrak{F}_{8,3}$, basta provarmos que $\mathfrak{F}_{8,3}$ não possui intersecção com as ações dos elementos $x^{\gamma}, y, x^{\gamma} y$, com $\gamma=1,2,3$, como mostraremos nos lemas a seguir. 
Lema 2.2.5 As rotações sobre $\Sigma_{j}, j=1,2$, determinadas pela ação dos elementos do grupo $\mathbb{Q}_{8}$ sobre $\mathfrak{F}_{8,3}$, satisfazem os seguintes itens:

1. $\operatorname{para} \gamma=1,2,3, \alpha\left(x^{\gamma}\right)\left(\beta_{1} * \beta_{2}\right)=R\left(\gamma \frac{\pi}{2}\right)\left(\beta_{1}\right) * R\left(-\gamma \frac{\pi}{2}\right)\left(\beta_{2}\right)$, com $0 \leq \gamma \frac{\pi}{2}<2 \pi$, isto é, as rotações determinadas por $x^{\gamma}$ sobre $\Sigma_{1}$ e $\Sigma_{2}$ são menores que $2 \pi$;

2. $\alpha(y)\left(\beta_{1} * \beta_{2}\right)=R(\pi)\left(\beta_{2}\right) * \beta_{1}$, isto é, as rotações determinadas por y sobre $\Sigma_{1} e \Sigma_{2}$ são menores que $2 \pi$;

3. para $\gamma=1,2,3, \alpha\left(x^{\gamma} y\right)\left(\beta_{1} * \beta_{2}\right)=R\left(\gamma \frac{\pi}{2}+\pi\right)\left(\beta_{2}\right) * R\left(-\gamma \frac{\pi}{2}\right)\left(\beta_{1}\right)$, com $0 \leq \gamma \frac{\pi}{2}+\pi<4 \pi$ e $0 \leq-\gamma \frac{\pi}{2}<2 \pi$, isto é, as rotações determinadas por $x^{\gamma} y$ sobre $\Sigma_{1}$ é menor que $4 \pi$ e sobre $\Sigma_{2}$ é menor que $2 \pi$.

$\underline{\text { Demonstração. }}$ 1. A rotação sobre $\Sigma_{1}$ determinada pela ação do elemento $x^{\gamma}$ sobre $\mathfrak{F}_{8,3}$ consiste em uma rotação de $\gamma \frac{\pi}{2}$ no sentido positivo (ou seja, anti-horário) no arco $A_{1}$, como pode ser visto na Observação 2.2.4. Logo,

$$
\gamma \frac{\pi}{2} \leq \gamma_{\max } \frac{\pi}{2}=3 \frac{\pi}{2}<4 \frac{\pi}{2}=2 \pi
$$

Dessa forma, a rotação determinada por $x^{\gamma}$ sobre $\Sigma_{1}$ é menor que $2 \pi$.

Agora, a rotação sobre $\Sigma_{2}$ determinada pela ação do elemento $x^{\gamma}$ sobre $\mathfrak{F}_{8,3}$ consiste em fazer uma rotação de $\gamma \frac{\pi}{2}$ no sentindo negativo no arco $A_{2}$. Analogamente a $\Sigma_{1}$, segue que a rotação determinada por $x^{\gamma}$ sobre $\Sigma_{2}$ é menor que $2 \pi$.

2. Agindo o elemento $y$ sobre $\mathfrak{F}_{8,3}$ podemos observar, pela equação (2.2.2), que a rotação sobre $\Sigma_{1}$ troca o arco $A_{1}$ de $\Sigma_{1}$ para $\Sigma_{2}$ sem nenhuma rotação. Logo, a rotação determinada pelo elemento $y$ sobre $\Sigma_{1}$ é menor que $2 \pi$.

Agora, a rotação sobre $\Sigma_{2}$ determinada pela ação do elemento y sobre $\mathfrak{F}_{8,3}$ consiste em trocar o arco $A_{2}$ de $\Sigma_{2}$ para $\Sigma_{1}$ e fazer uma rotação de $\pi$ no arco $A_{2}$ que agora encontra-se em $\Sigma_{1}$, como pode ser observado na equação (2.2.2). Assim, a rotação determinada por $y$ sobre $\Sigma_{2}$ é menor que $2 \pi$.

3. A rotação sobre $\Sigma_{1}$ determinada pela ação do elemento $x^{\gamma} y$ sobre $\mathfrak{F}_{8,3}$ consiste em trocar o arco $A_{2}$ de $\Sigma_{2}$ para $\Sigma_{1}$ e fazer uma rotação de $\gamma \frac{\pi}{2}+\pi$ no arco $A_{2}$, uma vez que a ação 
de $y$ sobre $\mathfrak{F}_{8,3}$ troca o arco $A_{2}$ de $\Sigma_{2}$ para $\Sigma_{1}$ rotacionando o arco $A_{2}$ de $\pi$, e a ação de $x^{\gamma}$ sobre $\mathfrak{F}_{8,3}$ é um rotação de $\gamma \frac{\pi}{2}$.

Como o valor máximo para $\gamma$ é $\max (\gamma)=3$ segue que a rotação máxima sobre $\Sigma_{1}$ determinada pela ação do elemento $x^{\gamma} y$ sobre $\mathfrak{F}_{8,3}$ é igual a

$$
\max (\gamma) \frac{\pi}{2}+\pi=5 \frac{\pi}{2}
$$

Assim, para qualquer $\gamma$ temos,

$$
\gamma \frac{\pi}{2}+\pi \leq \max (\gamma) \frac{\pi}{2}+\pi=5 \frac{\pi}{2}<4 \pi
$$

Portanto, a rotação determinada por $x^{\gamma} y$ sobre $\Sigma_{1}$ é menor que $4 \pi$.

Por outro lado, em $\Sigma_{2}, y$ apenas troca o arco de plano, prevalecendo a rotação do elemento $x^{\gamma}$, a qual já vimos no item 1 ser menor que $2 \pi$ em $\Sigma_{2}$.

Lema 2.2.6 As seguintes afirmações são verdadeiras:

1. $\operatorname{para} \gamma=1,2,3, \operatorname{Int}\left(\alpha\left(x^{\gamma}\right)\left(\mathfrak{F}_{8,3}\right)\right) \cap \operatorname{Int}\left(\mathfrak{F}_{8,3}\right)=\emptyset$;

2. $\operatorname{Int}\left(\alpha(y)\left(\mathfrak{F}_{8,3}\right)\right) \cap \operatorname{Int}\left(\mathfrak{F}_{8,3}\right)=\emptyset$;

3. $\operatorname{para} \gamma=1,2,3, \operatorname{Int}\left(\alpha\left(x^{\gamma} y\right)\left(\mathfrak{F}_{8,3}\right)\right) \cap \operatorname{Int}\left(\mathfrak{F}_{8,3}\right)=\emptyset$.

Demonstração. O método para provar estes fatos é sempre o mesmo: primeiro mostramos que as condições geométricas são equivalentes a algumas equações em $\mathbb{Z}$, e então que tais equações não possuem solução, por contradição.

1. Para provar esta afirmação lembremos que a ação do elemento $x$ não troca os arcos dos dois planos $\Sigma_{1}$ e $\Sigma_{2}$, e a rotação determinada por $x^{\gamma}$ é $\gamma \frac{\pi}{2}$. Dessa forma, por análises diretas da descrição geométrica de $\mathfrak{F}_{8,3}$ e pelo Lema 2.2.5,

$$
\operatorname{Int}\left(\alpha\left(x^{\gamma}\right)\left(\mathfrak{F}_{8,3}\right)\right) \cap \operatorname{Int}\left(\mathfrak{F}_{8,3}\right) \cap \Sigma_{1}=\operatorname{Int}\left(\alpha\left(x^{\gamma}\right)\left(A_{1}\right)\right) \cap \operatorname{Int}\left(A_{1}\right)
$$

é não vazia se $\gamma=0$. 
Portanto, $x^{\gamma}=x^{0}=1$ e dessa forma, segue que $\operatorname{Int}\left(\alpha\left(x^{\gamma}\right)\left(\mathfrak{F}_{8,3}\right)\right) \cap \operatorname{Int}\left(\mathfrak{F}_{8,3}\right)=\emptyset$, para $\gamma=1,2,3$.

2.

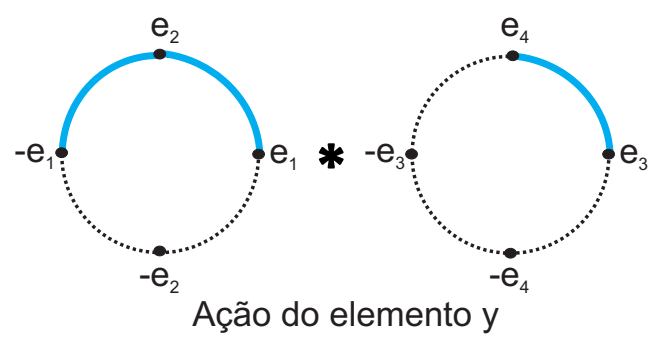

Pela figura acima (a qual denota a imagem da ação do elemento y sobre $\mathfrak{F}_{8,3}$ ) notamos que

$$
\operatorname{Int}\left(\alpha(y)\left(\mathfrak{F}_{8,3}\right)\right) \cap \operatorname{Int}\left(\mathfrak{F}_{8,3}\right) \cap \Sigma_{2}=\emptyset .
$$

Portanto, $\operatorname{Int}\left(\alpha(y)\left(\mathfrak{F}_{8,3}\right)\right) \cap \operatorname{Int}\left(\mathfrak{F}_{8,3}\right)=\emptyset$.

3. Para provar esta afirmação serão necessárias combinações das condições apresentadas a seguir, já que agora a ação dos elementos do grupo trocam os arcos de planos.

Analisemos os casos onde $\operatorname{Int}\left(\alpha\left(x^{\gamma} y\right)\left(\mathfrak{F}_{8,3}\right)\right) \cap \operatorname{Int}\left(\mathfrak{F}_{8,3}\right) \cap \Sigma_{1} \neq \emptyset$.

A rotação do elemento $x^{\gamma} y$ sobre $\Sigma_{1}$ é $[\gamma+2] \frac{\pi}{2}$. Lembremos que o arco $A_{2}$, após a ação de $x^{\gamma} y$ sobre $\mathfrak{F}_{8,3}$, está em $\Sigma_{1}$ e possui comprimento $\pi$, logo existem duas possibilidades para haver intersecção, as quais podem ocorrer quando:

$\left.1^{\circ}\right) \gamma+2=\theta$, onde $\theta=1,2$;

$\left.2^{\circ}\right) \gamma+2=4+\theta$, onde $\theta=1,2$.

Agora, analisemos os casos em que $\operatorname{Int}\left(\alpha\left(x^{\gamma} y\right)\left(\mathfrak{F}_{8,3}\right)\right) \cap \operatorname{Int}\left(\mathfrak{F}_{8,3}\right) \cap \Sigma_{2} \neq \emptyset$.

A rotação do elemento $x^{\gamma} y$ sobre $\Sigma_{2}$ é $-\gamma \frac{\pi}{2}$. Agora, o arco $A_{1}$ que está em $\Sigma_{2}$, após a ação de $x^{\gamma} y$ sobre $\mathfrak{F}_{8,3}$, possui comprimento $\frac{\pi}{2}$. Dessa forma, existe uma única possibilidade para haver intersecção, a qual denotaremos por terceiro caso.

$\left.3^{\circ}\right)-\gamma=-\theta^{\prime}$, onde $\theta^{\prime}=1,2$.

Notemos que todas as possibilidades são válidas, pois pelo item 2. do Lema 2.2.5, a rotação do elemento $x^{\gamma} y$ sobre $\Sigma_{1}$ e sobre $\Sigma_{2}$ são menores que $4 \pi$ e $2 \pi$, respectivamente. 
Novamente, lembremos que para $\operatorname{Int}\left(\alpha\left(x^{\gamma} y\right)\left(\mathfrak{F}_{8,3}\right)\right) \cap \operatorname{Int}\left(\mathfrak{F}_{8,3}\right)$ seja não vazia, deve haver intersecção nos dois arcos do 'curved join', ou seja, tanto no arco de $\Sigma_{1}$ quanto no arco do $\Sigma_{2}$, exceto pelos pontos do bordo do arco. Logo, devemos provar que não é possível encontrar $\gamma$ inteiro que satisfaça ao mesmo tempo as equações $1^{\circ}$ ) e $3^{\circ}$ ), ou $2^{\circ}$ ) e $3^{\circ}$ ).

Analisemos o $1^{\circ}$ ) e o $3^{\circ}$ ) casos.

Suponhamos que exista intersecção nos arcos de $\Sigma_{1}\left(1^{\circ}\right.$ caso $)$ e nos $\operatorname{arcos}$ de $\Sigma_{2}\left(3^{\circ}\right.$ caso $)$ ao mesmo tempo, ou seja, deve existir $\gamma$ inteiro que satisfaça o sistema abaixo:

$$
\left\{\begin{array}{l}
\gamma+2=\theta, \theta=1,2 \\
-\gamma=-\theta^{\prime}, \theta^{\prime}=1,2
\end{array}\right.
$$

Se $\theta=1$ então $\gamma=-1$, ou seja, $x^{\gamma} y=x^{-1} y=x^{3} y$.

Na primeira equação, se $\theta=2$ então $\gamma=0$ e como resultado temos o elemento $y$, que já foi analisado no item 2.

Agora, na segunda equação as únicas possibilidades para haver intersecção são $\gamma=1,2$, o que não satisfaz a primeira equação.

Logo, não existe $\gamma$ inteiro que satisfaça as duas equações.

Agora, analisemos o $2^{\circ}$ ) e o $3^{\circ}$ ) casos.

Suponhamos que exista intersecção nos arcos de $\Sigma_{1}\left(2^{\circ}\right.$ caso $)$ e nos $\operatorname{arcos}$ de $\Sigma_{2}\left(3^{\circ}\right.$ caso $)$ ao mesmo tempo, ou seja, deve existir $\gamma$ inteiro que satisfaça o seguinte sistema:

$$
\left\{\begin{array}{l}
\gamma+2=4+\theta, \theta=1,2 \\
-\gamma=-\theta^{\prime}, \theta^{\prime}=1,2
\end{array}\right.
$$

Na primeira equação, se $\theta=1$ então $\gamma=3$. E se $\theta=2$, temos $\gamma=4$, ou seja, $x^{\gamma} y=x^{4} y=y$, e o elemento $y$ já foi analisado no item 2.

Agora, na segunda equação as únicas possibilidades para haver intersecção são $\gamma=1,2$.

Assim, não existe $\gamma$ inteiro que satisfaça as duas equações.

Portanto, $\operatorname{Int}\left(\alpha\left(x^{\gamma} y\right)\left(\mathfrak{F}_{8,3}\right)\right) \cap \operatorname{Int}\left(\mathfrak{F}_{8,3}\right)=\emptyset$. 
Novamente, como $\mathfrak{F}_{24,3}$ está contida no interior de $\mathfrak{F}_{8,3}$, vista anteriormente, o Lema 2.2.5 e o Lema 2.2.6 seguem para $\mathfrak{F}_{24,3}$.

Resta então provarmos o seguinte resultado:

Lema 2.2.7 Para $\rho=1,2, \gamma=0,1,2,3$ e $\sigma=0,1$,

$$
\operatorname{Int}\left(\alpha\left(z^{\rho} x^{\gamma} y^{\sigma}\right)\left(\mathfrak{F}_{24,3}\right)\right) \cap \operatorname{Int}\left(\mathfrak{F}_{24,3}\right)=\emptyset .
$$

Demonstração. Mostraremos tal fato geometricamente, uma vez que a ação do elemento $z$ é uma rotação de $120^{\circ}$ no espaço, não podendo se visualizada em nenhum dos seis planos coordenados, como visto anteriormente no caso do $\mathbb{Q}_{8}$. Segue abaixo a ação desses elementos sobre $\mathfrak{F}_{24,3}$ :
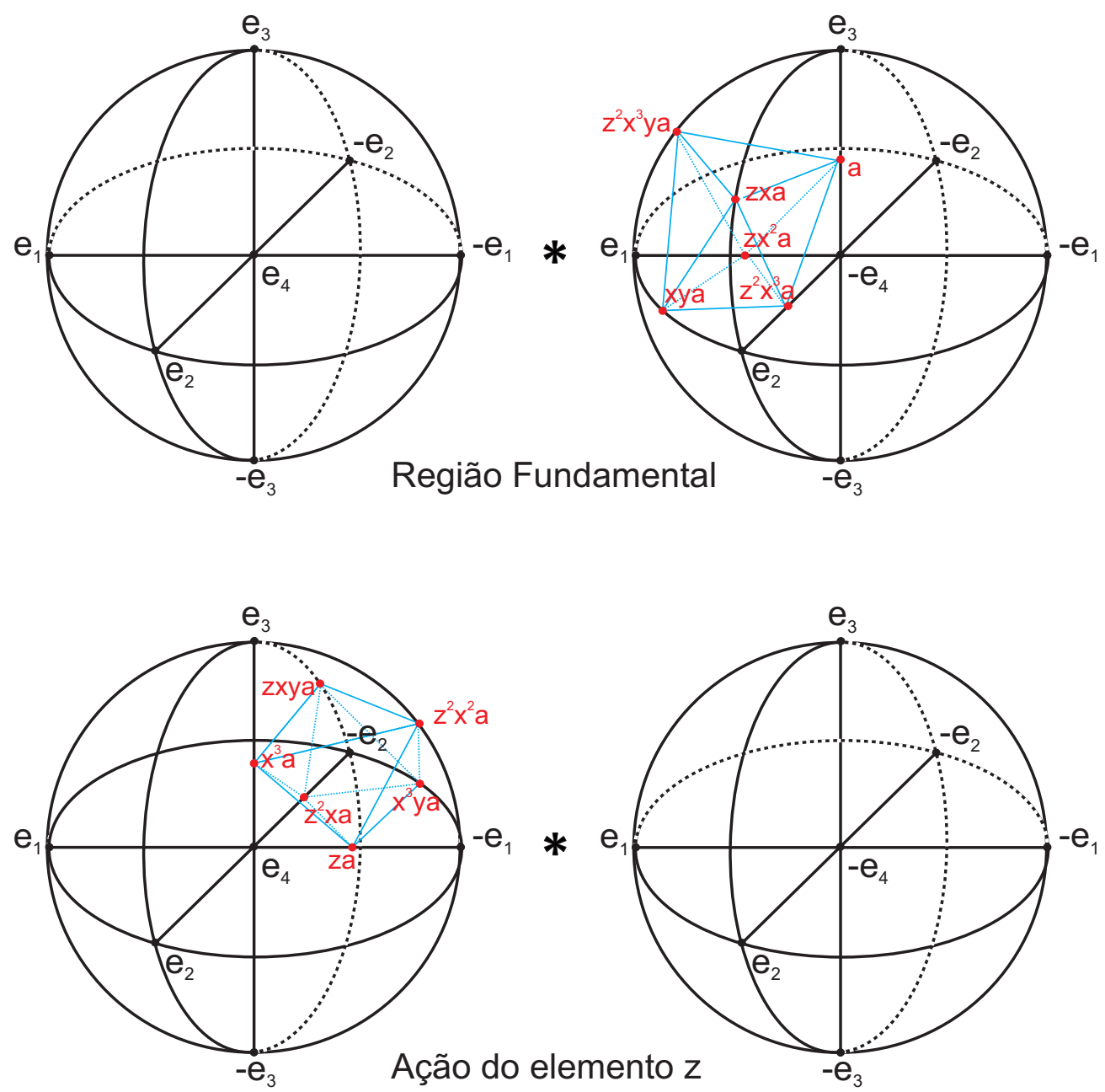

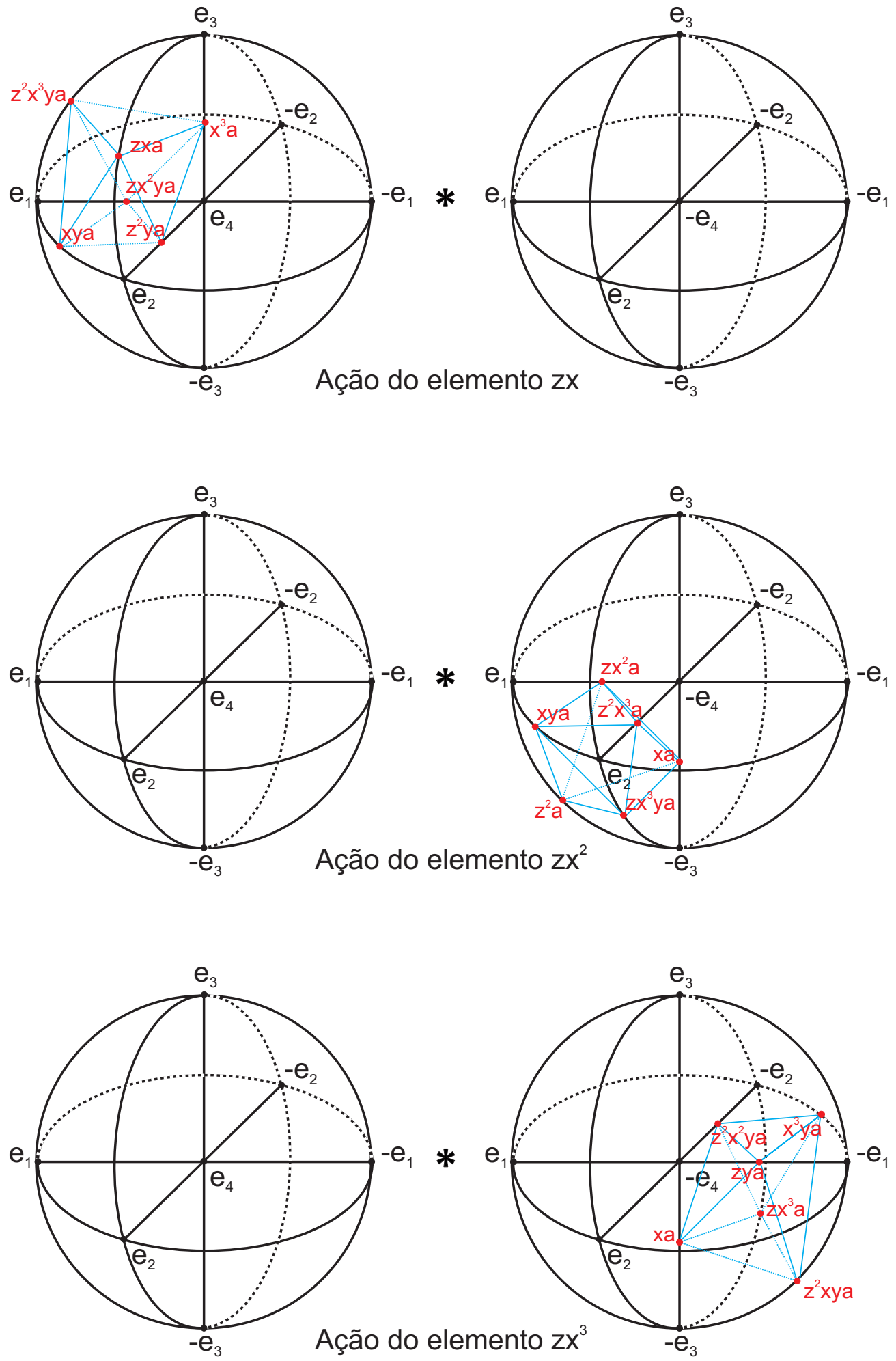

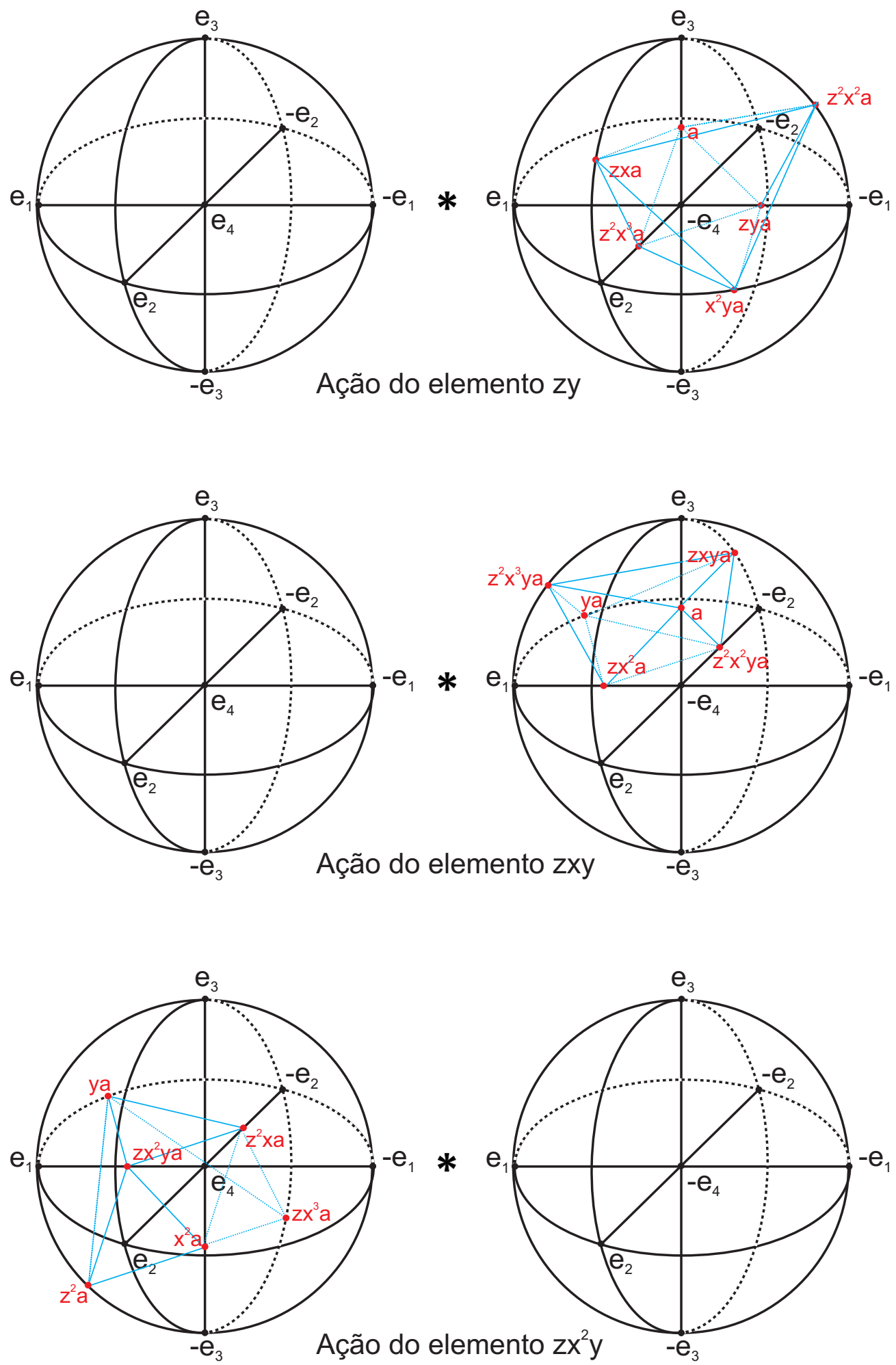

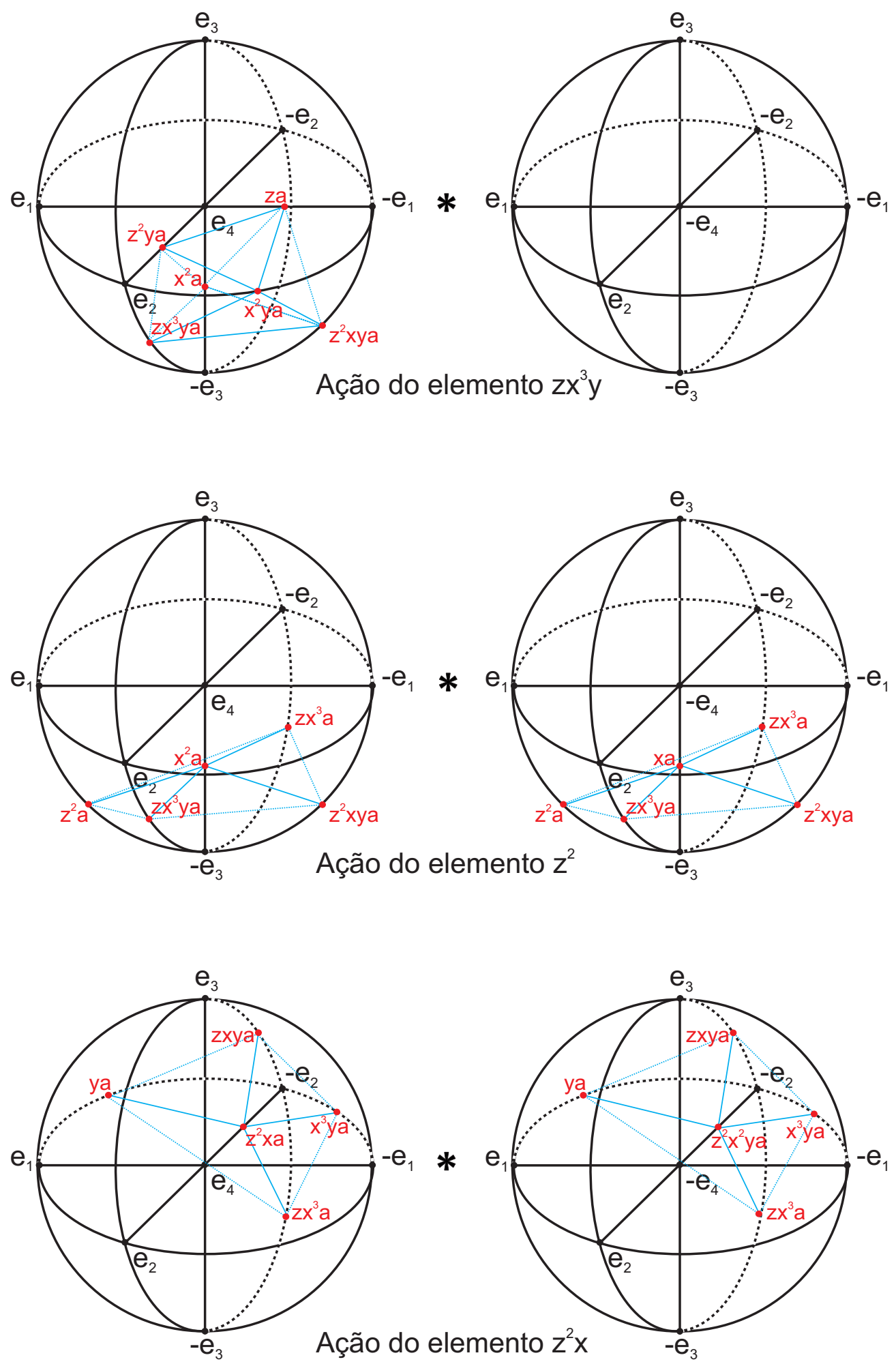

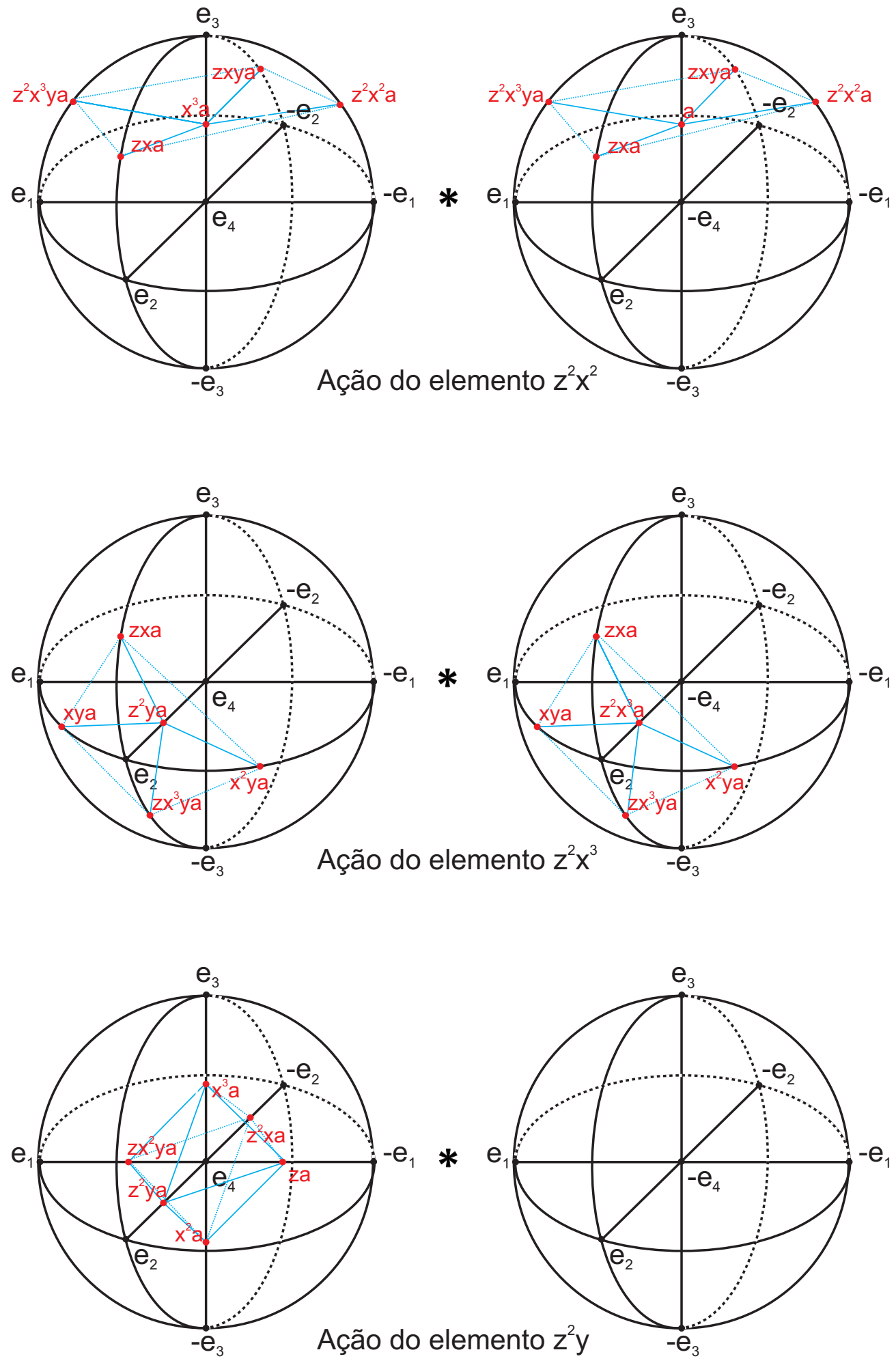

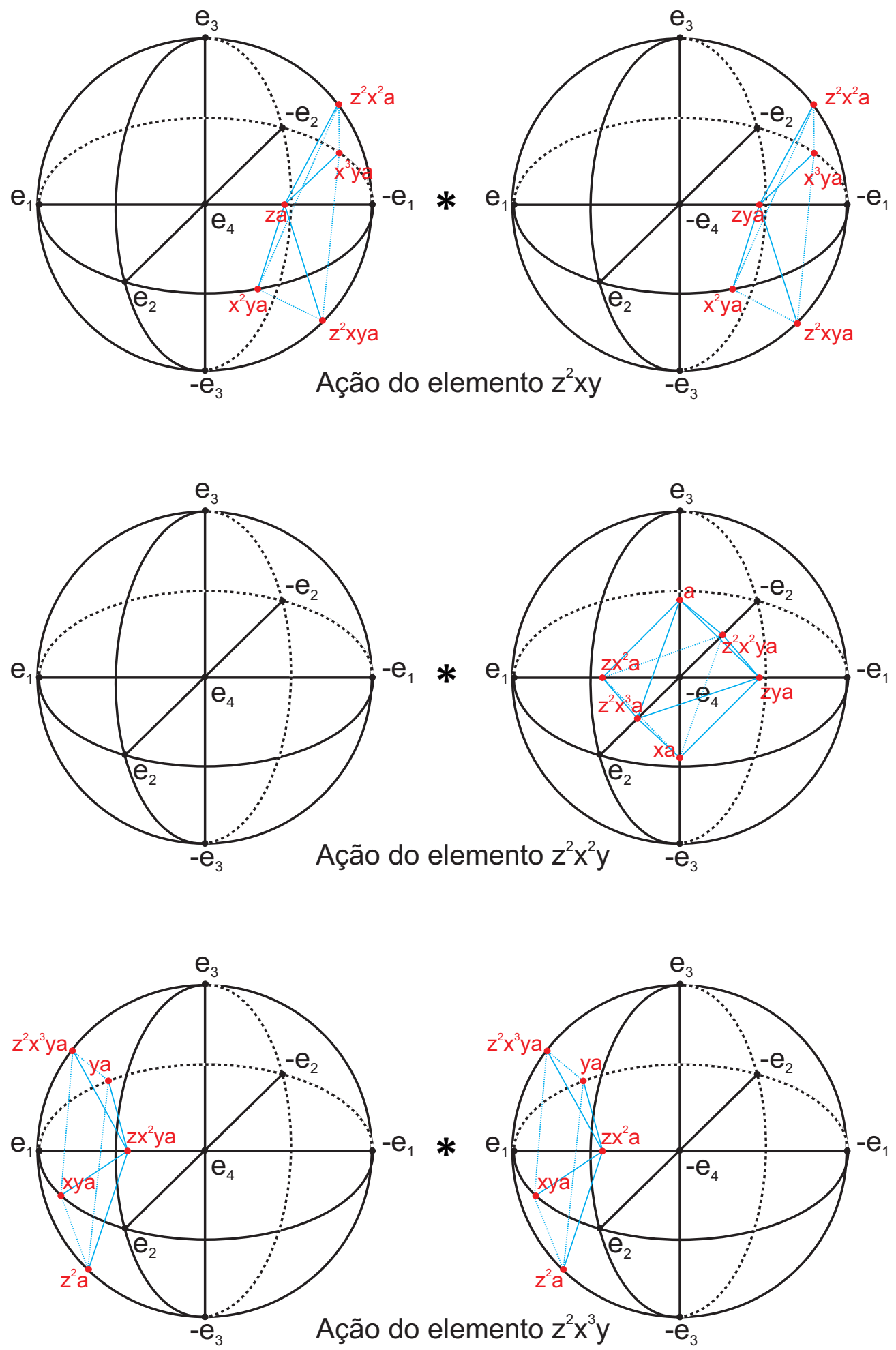

Facilmente, verificamos que não existe intersecção entre $\operatorname{Int}\left(\alpha\left(z^{\rho} x^{\gamma} y^{\sigma}\right)\left(\mathfrak{F}_{24,3}\right)\right)$ e $\operatorname{Int}\left(\mathfrak{F}_{24,3}\right)$, com $\rho=1,2, \gamma=0,1,2$ e $\sigma=0,1$.

Acabamos de provar então, o resultado a seguir: 
Proposição 2.2.8 Uma região (ou domínio) fundamental para a ação do grupo $P_{24}$ sobre $S^{3}$ via a representação $\alpha$ é $\mathfrak{F}_{24,3}=\left[a, z x a, z^{2} x^{3} a, x y a, z^{2} x^{3} y a, z x^{2} a\right]$.

\subsubsection{Decomposição celular e complexo de cadeias}

Se $X=S^{n} / G$, com $G$ algum grupo finito agindo por isometria, $\mathfrak{F}$ é a região fundamental da ação de $G$, e $q: S^{n} \rightarrow X$ é a aplicação quociente natural, então $q$ é uma aplicação de recobrimento, $S^{n}=\widetilde{X}$ é o espaço de recobrimento universal de $X,\left.q\right|_{\mathfrak{F}}: \mathfrak{F} \rightarrow X$ é sobrejetiva e é um homeomorfismo relativo para o par $(\mathfrak{F}, \partial \mathfrak{F}) \rightarrow\left(X, X^{(n-1)}\right)$, onde $X^{(n-1)}$ é o $(n-1)$-esqueleto de $X$ (lembrando que $X$ é uma variedade compacta). Uma decomposição celular $G$-equivariante $\widetilde{K}$ de $S^{n}$ determina uma decomposição celular $\widetilde{L}$ de $\mathfrak{F}$, onde $\widetilde{L}$ é um subcomplexo de $\widetilde{K}, \widetilde{K}=\pi \widetilde{L}$ e $q(\widetilde{L})$ resultará em uma decomposição $L$ de $X$. Além disso, no mínimo um levantamento de cada célula de $L$ será colocado em $\widetilde{L}$. Dessa forma, podemos escolher para cada célula $c \in L$ uma única célula $\widetilde{c} \in \widetilde{L}$, a qual será chamada um representante do levantamento de $c$. Como todas as outras células de $\widetilde{L}$ estão na órbita de $G$ para alguma célula $\widetilde{c}$ (isto é, estão na órbita de $G$ pela ação de algum elemento do grupo), o complexo celular de $\widetilde{L}$ pode ser descrito usando as células $\widetilde{c}$ e algumas ações de $G$ sobre $\widetilde{c}$. Este conjunto de células será chamado conjunto minimal de representantes do levantamento, o qual resultará em uma decomposição celular minimal de $\mathfrak{F}$, que denotaremos por $\widetilde{Z}$. É claro que $q(\widetilde{Z})=L$. Tomando todas as órbitas completas de células em $\widetilde{Z}$, obtemos o complexo celular $\widetilde{K}=G \widetilde{Z}$, que é uma decomposição celular $G$-equivariante de $S^{n}$.

Passaremos agora a determinar uma $P_{24}$ decomposição celular equivariante de $S^{3}$. Para isso, definimos uma decomposição celular de $S^{3}$ do seguinte modo: fixado um ponto de $S^{3}$, e identificando esse ponto com $a$, as 0-células de $S^{3}$ são os elementos das órbitas $P_{24} a$. As 1-células, 2-células e 3-células de $S^{3}$ são as 1-células, 2-células e 3-célula, respectivamente, da região fundamental transladadas pela ação dos elementos do grupo $P_{24}$. Seja $\widetilde{L}$ uma decomposição celular de $\mathfrak{F}_{24,3}$. Note que $\widetilde{K}=P_{24} \widetilde{L}$ é uma $P_{24}$ decomposição celular de $S^{3}$. Isto resulta no seguinte lema: 
Lema 2.2.9 Uma $P_{24}$ decomposição celular equivariante de $S^{3}$ é $\widetilde{K}=P_{24} \widetilde{L}$, onde $\widetilde{L}$ foi definido anteriormente. O subcomplexo $\widetilde{L}$ é uma decomposição celular de $\mathfrak{F}_{24,3}$. O quociente $L=q(\widetilde{L})=\widetilde{L} / \alpha\left(P_{24}\right)$ resulta em uma decomposição celular de $\mathscr{P}_{3}$.

Descreveremos agora, um conjunto minimal de levantamentos $\widetilde{Z}$ das células de $L$ em $S^{3}$, e seus operadores bordos. Para descrever tal complexo, usaremos a seguinte notação: fixando uma 0-célula, $a=\left(0,0, \frac{\sqrt{2}}{2},-\frac{\sqrt{2}}{2}\right)$, denotamos as outras 0-células pelas ações dos elementos do grupo. No caso de dimensões maiores as células são obtidas pelo 'join' das de dimensão menor.

Proposição 2.2.10 Um conjunto minimal $\widetilde{Z}$ de representantes do levantamento $\widetilde{c}_{q, s}$ (onde o primeiro índice denota a dimensão) das células da decomposição celular $L$ de $\mathscr{P}_{3}$, definidas no Lema 2.2.9, é:

$$
\begin{aligned}
& \widetilde{c}_{0,1}=a=\left(0,0, \frac{\sqrt{2}}{2},-\frac{\sqrt{2}}{2}\right), \\
& \widetilde{c}_{1,1}=\left[a, z^{2} x^{3} y a\right], \\
& \widetilde{c}_{1,2}=[a, z x a], \\
& \widetilde{c}_{1,3}=\left[a, z^{2} x^{3} a\right], \\
& \widetilde{c}_{1,4}=\left[a, z x^{2} a\right], \\
& \widetilde{c}_{2,1}=\left[a, z x a, z^{2} x^{3} a\right], \\
& \widetilde{c}_{2,2}=\left[a, z x^{2} a, z^{2} x^{3} y a\right], \\
& \widetilde{c}_{2,3}=\left[a, z x^{2} a, z^{2} x^{3} a\right], \\
& \widetilde{c}_{2,4}=\left[a, z x a, z^{2} x^{3} y a\right], \\
& \widetilde{c}_{3,1}=\left[a, z x a, z^{2} x^{3} a, x y a, z^{2} x^{3} y a, z x^{2} a\right],
\end{aligned}
$$

onde os operadores bordos são:

$$
\begin{aligned}
& \partial_{1}\left(\widetilde{c}_{1,1}\right)=\left(z^{2} x^{3} y-1\right) \widetilde{c}_{0,1}, \\
& \partial_{1}\left(\widetilde{c}_{1,2}\right)=(z x-1) \widetilde{c}_{0,1}, \\
& \partial_{1}\left(\widetilde{c}_{1,3}\right)=\left(z^{2} x^{3}-1\right) \widetilde{c}_{0,1},
\end{aligned}
$$




$$
\begin{aligned}
& \partial_{1}\left(\widetilde{c}_{1,4}\right)=\left(z x^{2}-1\right) \widetilde{c}_{0,1}, \\
& \partial_{2}\left(\widetilde{c}_{2,1}\right)=-z^{2} x^{3} \widetilde{c}_{1,1}+\widetilde{c}_{1,2}-\widetilde{c}_{1,3}, \\
& \partial_{2}\left(\widetilde{c}_{2,2}\right)=-\widetilde{c}_{1,1}-z^{2} x^{3} y \widetilde{c}_{1,3}+\widetilde{c}_{1,4}, \\
& \partial_{2}\left(\widetilde{c}_{2,3}\right)=-z x^{2} \widetilde{c}_{1,2}+\widetilde{c}_{1,3}-\widetilde{c}_{1,4}, \\
& \partial_{2}\left(\widetilde{c}_{2,4}\right)=\widetilde{c}_{1,1}-\widetilde{c}_{1,2}-z x \widetilde{c}_{1,4}, \\
& \partial_{3}\left(\widetilde{c}_{3,1}\right)=\left(1-z^{2} x^{3} y\right) \widetilde{c}_{2,1}+\left(1-z^{2} x^{3}\right) \widetilde{c}_{2,2}+(1-z x) \widetilde{c}_{2,3}+\left(1-z x^{2}\right) \widetilde{c}_{2,4} .
\end{aligned}
$$

Demonstração. A prova dessa proposição é geométrica e é baseada em uma descrição explícita da decomposição celular $\widetilde{L}$ da região fundamental.

Observando a região fundamental (mostrada na figura abaixo), obtemos o seguinte conjunto de células:

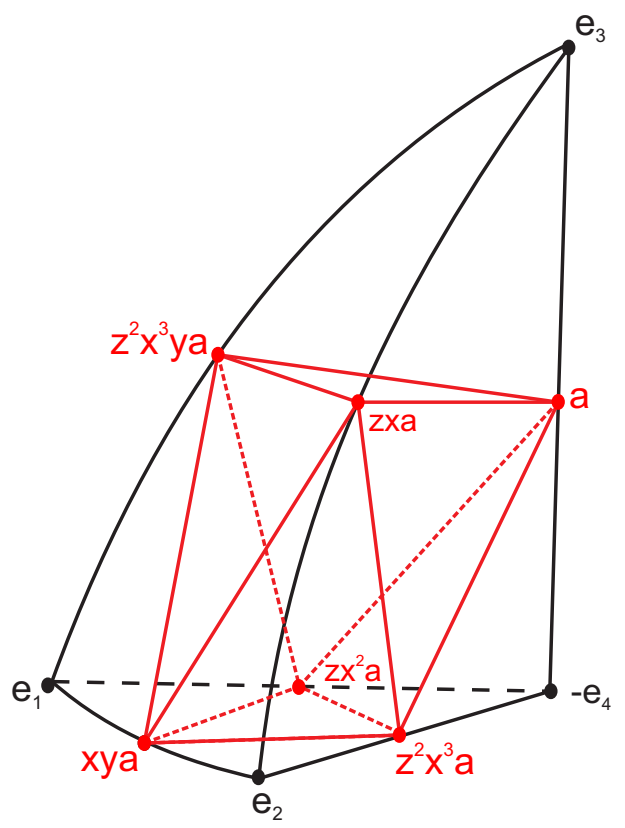

as 0-células são os vértices de $\mathfrak{F}_{24,3}$, isto é, os pontos:

$$
c_{0,1}=a, \quad c_{0,2}=z x a, \quad c_{0,3}=z^{2} x^{3} a, \quad c_{0,4}=z^{2} x^{3} y a, \quad c_{0,5}=z x^{2} a, \quad c_{0,6}=x y a ;
$$

as 1-células são os arcos:

$$
\begin{aligned}
& c_{1,1}=\left[a, z^{2} x^{3} a\right], \quad c_{1,2}=[a, z x a], \quad c_{1,3}=\left[a, z x^{2} a\right], \quad c_{1,4}=\left[z^{2} x^{3} a, z x a\right], \\
& c_{1,5}=[z x a, x y a], \quad c_{1,6}=\left[z^{2} x^{3} a, x y a\right], \quad c_{1,7}=\left[z^{2} x^{3} y a, x y a\right], \quad c_{1,8}=\left[z x a, z^{2} x^{3} y a\right], \\
& c_{1,9}=\left[a, z^{2} x^{3} y a\right], \quad c_{1,10}=\left[z^{2} x^{3} y a, z^{2} x a\right] \quad c_{1,11}=\left[z x^{2} a, x y a\right], \quad c_{1,12}=\left[z x^{2} a, z^{2} x^{3} a\right] ;
\end{aligned}
$$


e as 2-células são os conjuntos:

$$
\begin{aligned}
& c_{2,1}=\left[a, z x a, z^{2} x^{3} a\right], \quad c_{2,2}=\left[z x a, x y a, z^{2} x^{3} a\right], \quad c_{2,3}=\left[z x a, z^{2} x^{3} y a, x y a\right], \\
& c_{2,4}=\left[z^{2} x^{3} y a, x y a, z x^{2} a\right], \quad c_{2,5}=\left[z^{2} x^{3} y a, z x^{2} a, a\right], \quad c_{2,6}=\left[a, z x^{2} a, z^{2} x^{3} a\right], \\
& c_{2,7}=\left[a, z x a, z^{2} x^{3} y a\right], \quad c_{2,8}=\left[z x^{2} a, z^{2} x^{3} a, x y a\right] .
\end{aligned}
$$

Podemos verificar que a união das oito 2-células, apresentadas anteriormente, coincide com o bordo da região fundamental, $\partial \mathfrak{F}_{24,3}$, que o bordo de cada 2-célula está contido na união de 1-células, e que o bordo de cada 1-célula está contido na união de 0-células. Isso mostra que este conjunto de células fornece uma decomposição celular da região fundamental. Agora, usando as ações do grupo, mostraremos que todas as células são dadas por combinações $\mathbb{Z} P_{24}$ lineares do conjunto minimal de células, $\widetilde{Z}$, dado no enunciado da proposição. Note que tal conjunto minimal é naturalmente sugerido uma vez que fixemos um ponto inicial, que é a 0-célula.

Fixemos o levantamento $\widetilde{c}_{0,1}$ de $c_{0,1}$ como 0-célula minimal, ou seja, consideremos $\widetilde{c}_{0,1}=a$ como 0-célula de $\widetilde{Z}$. As outras 0-células são descritas por elementos do grupo agindo sobre $\widetilde{c}_{0,1}$, como mostra a figura a seguir:

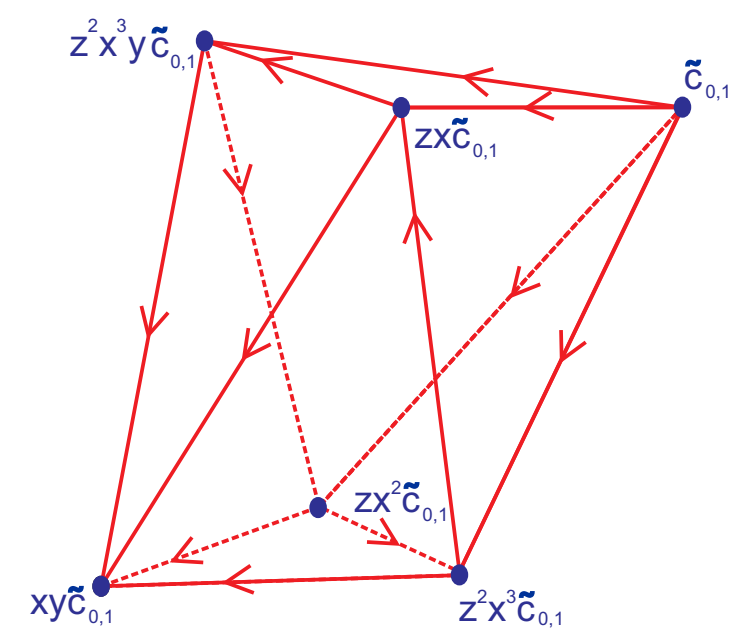

Quanto as 1-células, temos as seguintes relações entre elas:

$$
\begin{aligned}
& c_{1,4}=z^{2} x^{3} c_{1,9}, \quad c_{1,5}=z x c_{1,1}, \quad c_{1,6}=z^{2} x^{3} c_{1,3}, \quad c_{1,7}=z^{2} x^{3} y c_{1,2}, \\
& c_{1,8}=z x c_{1,3}, \quad c_{1,10}=z^{2} x^{3} y c_{1,1}, \quad c_{1,11}=z x^{2} c_{1,9}, \quad c_{1,12}=z x^{2} c_{1,2}
\end{aligned}
$$

Assim, escolhemos como conjunto minimal de levantamentos de 1-células, o seguinte 
conjunto:

$$
\begin{gathered}
\widetilde{c}_{1,1}=c_{1,9}=\left[a, z^{2} x^{3} y a\right], \quad \widetilde{c}_{1,2}=c_{1,2}=[a, z x a], \\
\widetilde{c}_{1,3}=c_{1,1}=\left[a, z^{2} x^{3} a\right], \quad \widetilde{c}_{1,4}=c_{1,3}=\left[a, z x^{2} a\right] .
\end{gathered}
$$

Nas figuras abaixo, temos as correspondências entre cada um dos levantamentos escolhidos e as 1-células relacionadas a ele.
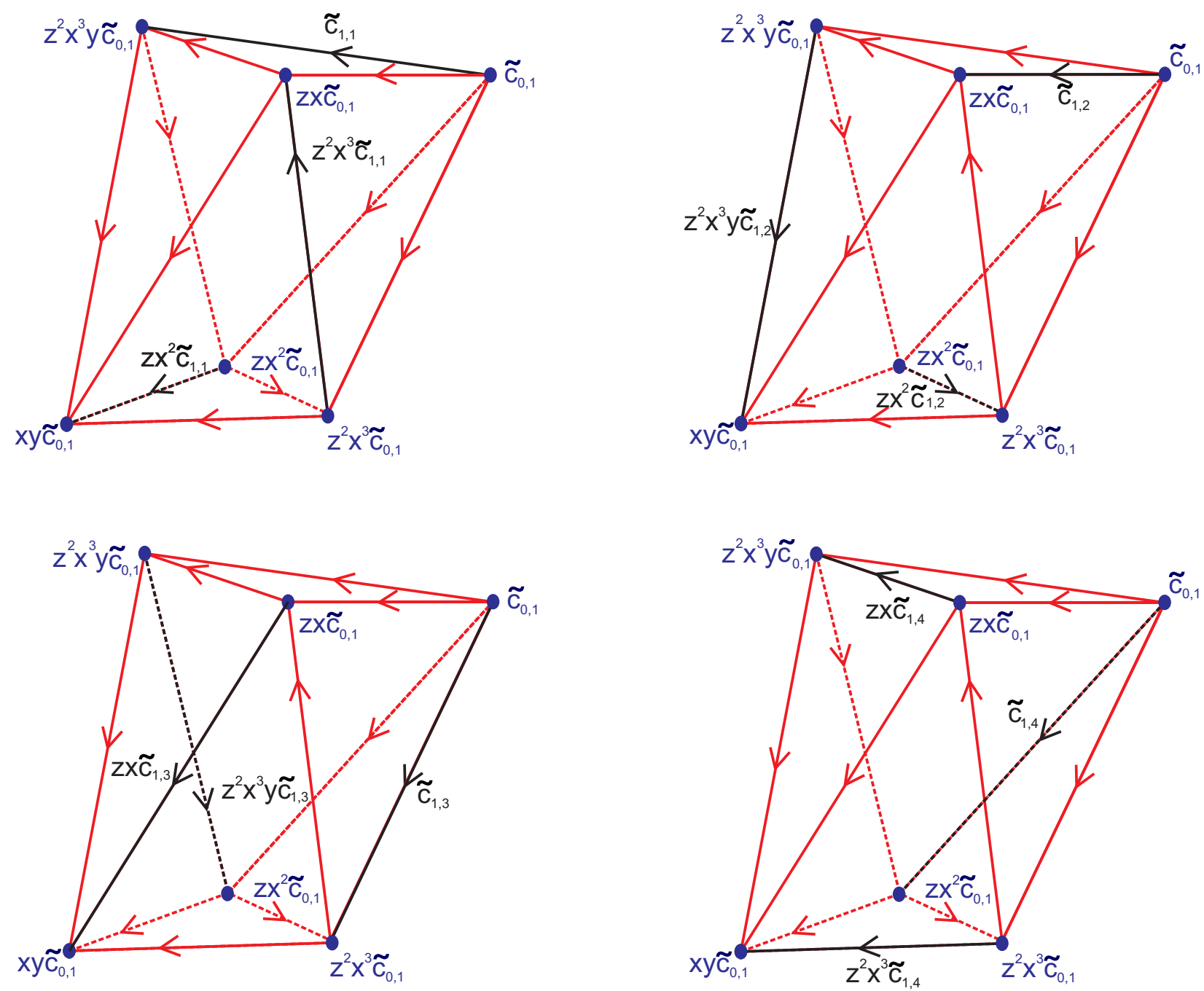

Para uma visualização mais geral, a figura a seguir mostra a região fundamental com suas 0-células e 1-células em função dos levantamentos minimais escolhidos em cada dimensão. 


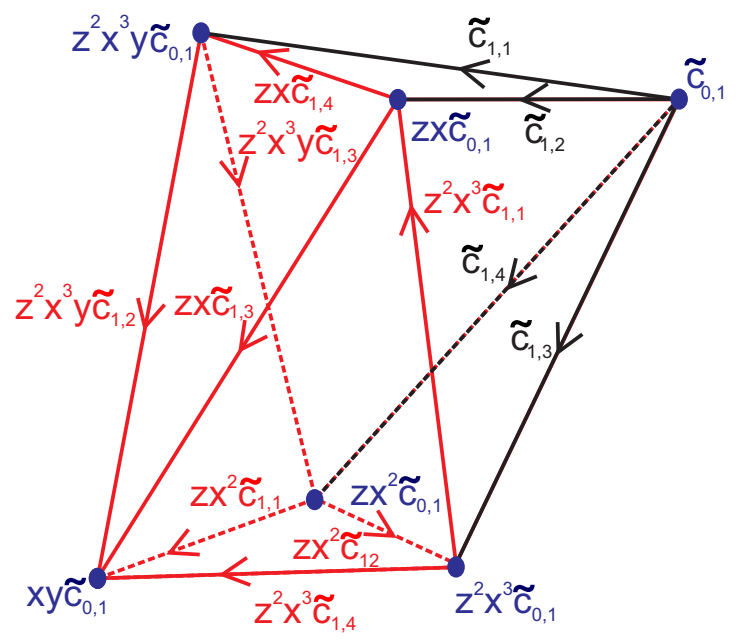

Passaremos agora a escolher as 2-células de $\widetilde{Z}$. O processo é similar ao que já foi feito anteriormente. Identificando as órbitas das 2-células em $\widetilde{L}$, obtemos:

$$
c_{2,2}=z^{2} x^{3} c_{2,5}, \quad c_{2,3}=z x c_{2,6}, \quad c_{2,4}=z^{2} x^{3} y c_{2,1}, \quad c_{2,8}=z x^{2} c_{2,7} .
$$

Dessa forma, escolhemos como conjunto minimal de levantamentos de 2-células, o seguinte conjunto:

$$
\begin{aligned}
\widetilde{c}_{2,1}=c_{2,1}=\left[a, z x a, z^{2} x^{3} a\right], & \widetilde{c}_{2,2}=c_{2,5}=\left[z^{2} x^{3} y a, z x^{2} a, a\right], \\
\widetilde{c}_{2,3}=c_{2,6}=\left[a, z x^{2} a, z^{2} x^{3} a\right], & \widetilde{c}_{2,4}=c_{2,7}=\left[a, z x a, z^{2} x^{3} y a\right] .
\end{aligned}
$$

Nas figuras abaixo, apresentamos as correspondências entre as 2-células em relação aos levantamentos escolhidos.
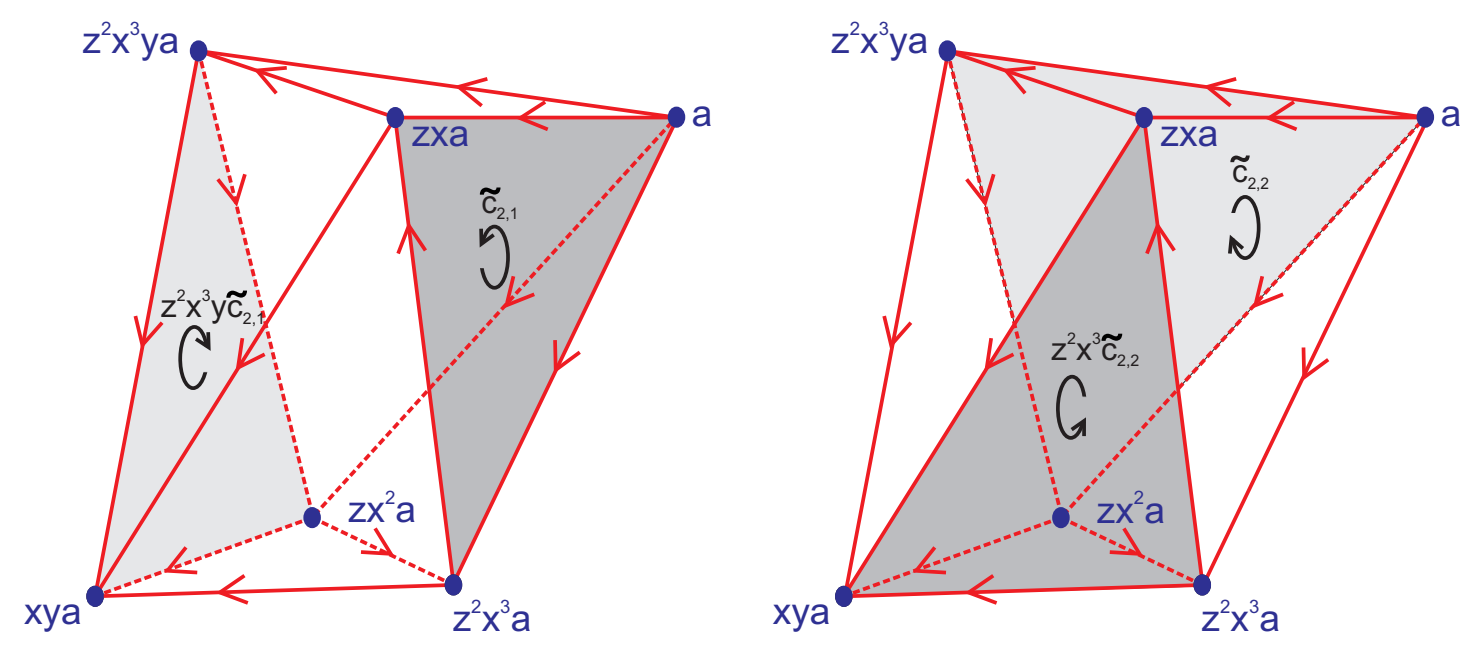

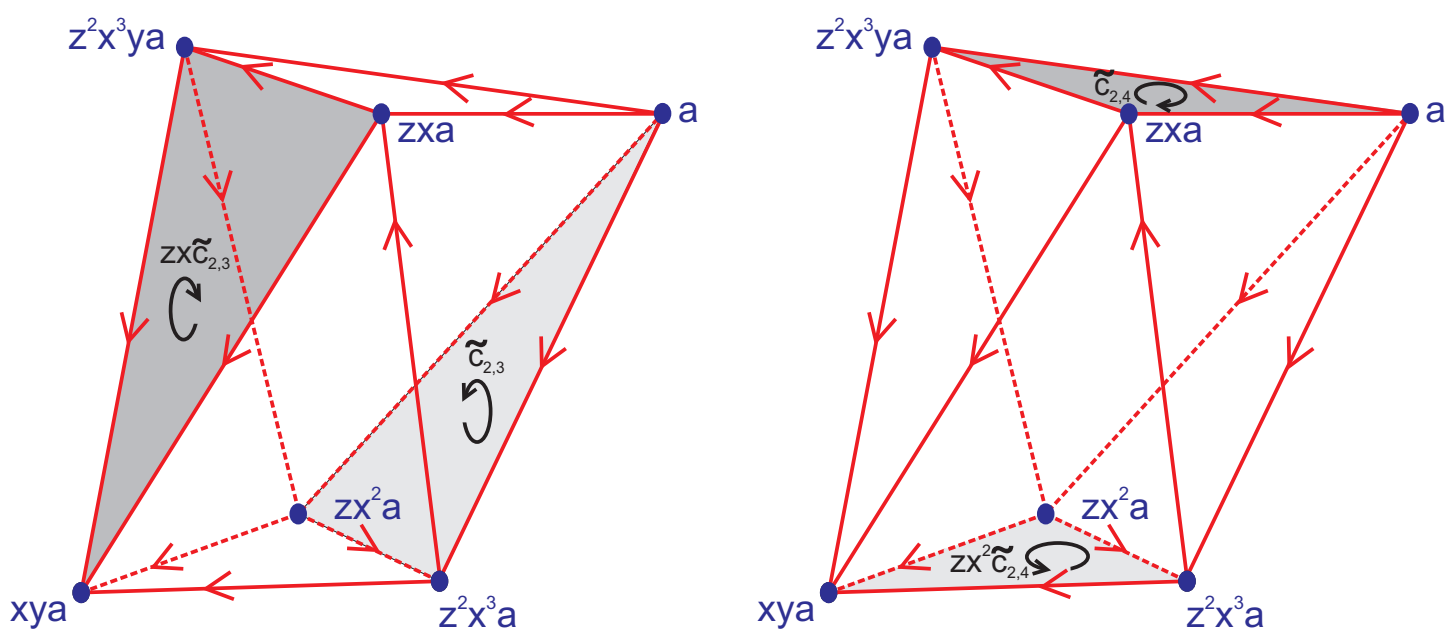

Na figura a seguir são mostrados apenas os levantamentos minimais em dimensão dois.

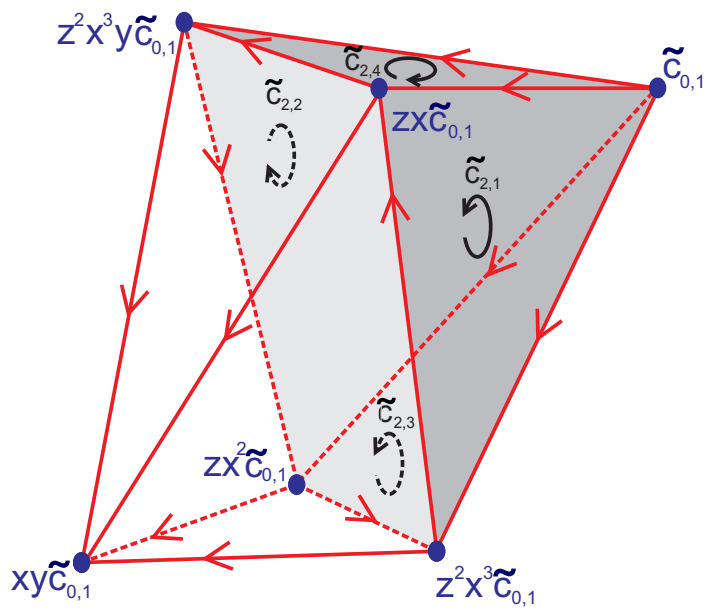

Agora, não é difícil ver que a única 3-célula de $\widetilde{Z}$ é $\widetilde{c}_{3,1}=\left[a, z x a, z^{2} x^{3} a, x y a, z^{2} x^{3} y a, z x^{2} a\right]$ e ela coincide com toda a região fundamental.

A figura a seguir mostra a região fundamental com todas as suas células relativas as células de $\widetilde{Z}$.

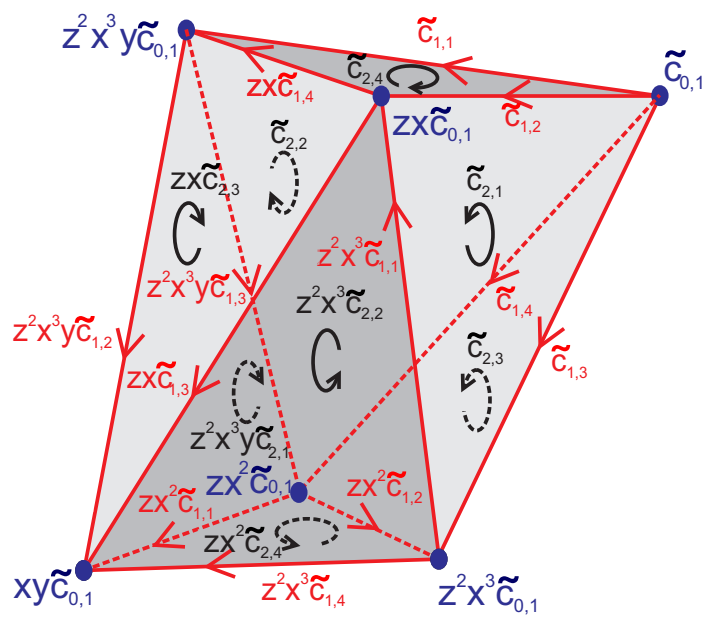


Finalmente, encontramos os operadores bordos geometricamente e escrevendo os coeficientes em $\mathbb{Z} P_{24}$, usando as descrições conhecidas da órbita do ponto a. Isto completa a prova.

Podemos agora apresentar o complexo de cadeias do espaço de recobrimento universal da forma espacial esférica tetraedral $\mathscr{P}_{3}=\frac{S^{3}}{\alpha\left(P_{24}\right)}$.

Seja $C(\widetilde{K} ; \mathbb{Z})$ um complexo com coeficientes inteiros. A ação do grupo das transformações de recobrimento faz de cada grupo de cadeia $C_{q}(\widetilde{K} ; \mathbb{Z})$ um módulo sobre o anel grupo $\mathbb{Z} \pi_{1}(X)$, e cada um desses módulos é $\mathbb{Z} \pi_{1}(X)$-livre [17, pag.377] e finitamente gerado pela escolha natural de $q$-células de $L$. Como $L$ é finito, segue que $C(\widetilde{K} ; \mathbb{Z})$ é livre e finitamente gerado sobre $\mathbb{Z} \pi_{1}(X)$. Obtemos assim, um complexo de módulos livres e finitamente gerados sobre $\mathbb{Z} \pi_{1}(X)$, o qual denotaremos por $C\left(\widetilde{K} ; \mathbb{Z} \pi_{1}(X)\right)$.

Usando a decomposição celular e os operadores bordos de cada célula, descritos na proposição anterior, obtemos o seguinte resultado:

Teorema 2.2.11 O complexo de cadeias $C\left(\mathscr{P}_{3} ; \mathbb{Z} P_{24}\right)$ do espaço de recobrimento universal da forma espacial esférica tetraedral, com a ação do grupo fundamental atuando por transformações de recobrimento, é o seguinte complexo de $\mathbb{Z} P_{24}$ módulos livres e finitamente gerados:

$$
0 \longrightarrow C_{3} \stackrel{\partial_{3}}{\longrightarrow} C_{2} \stackrel{\partial_{2}}{\longrightarrow} C_{1} \stackrel{\partial_{1}}{\longrightarrow} C_{0} \longrightarrow 0,
$$

onde

$$
\begin{aligned}
C_{0} & =\mathbb{Z} P_{24}\left[\widetilde{c}_{0,1}\right], \\
C_{1} & =\mathbb{Z} P_{24}\left[\widetilde{c}_{1,1}, \widetilde{c}_{1,2}, \widetilde{c}_{1,3}, \widetilde{c}_{1,4}\right], \\
C_{2} & =\mathbb{Z} P_{24}\left[\widetilde{c}_{2,1}, \widetilde{c}_{2,2}, \widetilde{c}_{2,3}, \widetilde{c}_{2,4}\right], \\
C_{3} & =\mathbb{Z} P_{24}\left[\widetilde{c}_{3,1}\right],
\end{aligned}
$$

com os operadores bordos dados por:

$$
\begin{aligned}
& \partial_{1}\left(\widetilde{c}_{1,1}\right)=\left(z^{2} x^{3} y-1\right) \widetilde{c}_{0,1}, \\
& \partial_{1}\left(\widetilde{c}_{1,2}\right)=(z x-1) \widetilde{c}_{0,1}, \\
& \partial_{1}\left(\widetilde{c}_{1,3}\right)=\left(z^{2} x^{3}-1\right) \widetilde{c}_{0,1},
\end{aligned}
$$




$$
\begin{aligned}
& \partial_{1}\left(\widetilde{c}_{1,4}\right)=\left(z x^{2}-1\right) \widetilde{c}_{0,1}, \\
& \partial_{2}\left(\widetilde{c}_{2,1}\right)=-z^{2} x^{3} \widetilde{c}_{1,1}+\widetilde{c}_{1,2}-\widetilde{c}_{1,3}, \\
& \partial_{2}\left(\widetilde{c}_{2,2}\right)=-\widetilde{c}_{1,1}-z^{2} x^{3} y \widetilde{c}_{1,3}+\widetilde{c}_{1,4}, \\
& \partial_{2}\left(\widetilde{c}_{2,3}\right)=-z x^{2} \widetilde{c}_{1,2}+\widetilde{c}_{1,3}-\widetilde{c}_{1,4}, \\
& \partial_{2}\left(\widetilde{c}_{2,4}\right)=\widetilde{c}_{1,1}-\widetilde{c}_{1,2}-z x \widetilde{c}_{1,4}, \\
& \partial_{3}\left(\widetilde{c}_{3,1}\right)=\left(1-z^{2} x^{3} y\right) \widetilde{c}_{2,1}+\left(1-z^{2} x^{3}\right) \widetilde{c}_{2,2}+(1-z x) \widetilde{c}_{2,3}+\left(1-z x^{2}\right) \widetilde{c}_{2,4} .
\end{aligned}
$$

Tal complexo é exato em todas as dimensões do meio, isto é, $\operatorname{Im}\left(\partial_{j+1}\right)=\operatorname{Ker}\left(\partial_{j}\right)$, para $j=0,1,2$.

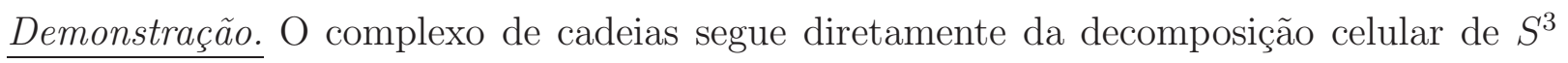
descrita na Proposição 2.2.10, vista anteriormente.

Afirmação 2.2.1 O complexo de cadeias do teorema é semi-exato.

Demonstração. Temos

$$
\partial_{1} \partial_{2}=\left(\begin{array}{cccc}
-z^{2} x^{3} & 1 & -1 & 0 \\
-1 & 0 & -z^{2} x^{3} y & 1 \\
0 & -z x^{2} & 1 & -1 \\
1 & -1 & 0 & -z x
\end{array}\right) \cdot\left(\begin{array}{c}
z^{2} x^{3} y-1 \\
z x-1 \\
z^{2} x^{3}-1 \\
z x^{2}-1
\end{array}\right)=\left(\begin{array}{l}
0 \\
0 \\
0 \\
0
\end{array}\right)
$$

De fato,

- $-z^{2} x^{3}\left(z^{2} x^{3} y-1\right)+(z x-1)-\left(z^{2} x^{3}-1\right)=-z^{2} x^{3} z^{2} x^{3} y+z^{2} x^{3}+z x-1-z^{2} x^{3}+1$ $=-z^{2} x^{2} y z y z x^{3} y+z x=-z^{2} z x y y^{2} z x^{3} y+z x=-x y z x^{2} x^{3} y+z x=-z y x y+z x=-z x+z x$ $=0$.

- $-\left(z^{2} x^{3} y-1\right)-z^{2} x^{3} y\left(z^{2} x^{3}-1\right)+\left(z x^{2}-1\right)=-z^{2} x^{3} y+1-z^{2} x^{3} y z^{2} x^{3}+z^{2} x^{3} y+z x^{2}-1$ $=-z^{2} x y y z y z y x^{3}+z x^{2}=-z^{2} x y z x y z x^{3} y^{3}+z x^{2}=-z^{2} z y x y z x y+z x^{2}=-y z y x y+z x^{2}$ $=-z x^{2}+z x^{2}=0$.

- $-z x^{2}(z x-1)+\left(z^{2} x^{3}-1\right)-\left(z x^{2}-1\right)=-z x^{2} z x+z x^{2}+z^{2} x^{3}-1-z x^{2}+1=-z z x^{2} x+z^{2} x^{3}$ $=-z^{2} x^{3}+z^{2} x^{3}=0$. 
- $\left(z^{2} x^{3} y-1\right)-(z x-1)-z x\left(z x^{2}-1\right)=z^{2} x^{3} y-1-z x+1-z x z x^{2}+z x=z^{2} x^{3} y-z z x y x^{2}$ $=z^{2} x^{3} y-z^{2} x x^{2} y=z^{2} x^{3} y-z^{2} x^{3} y=0$.

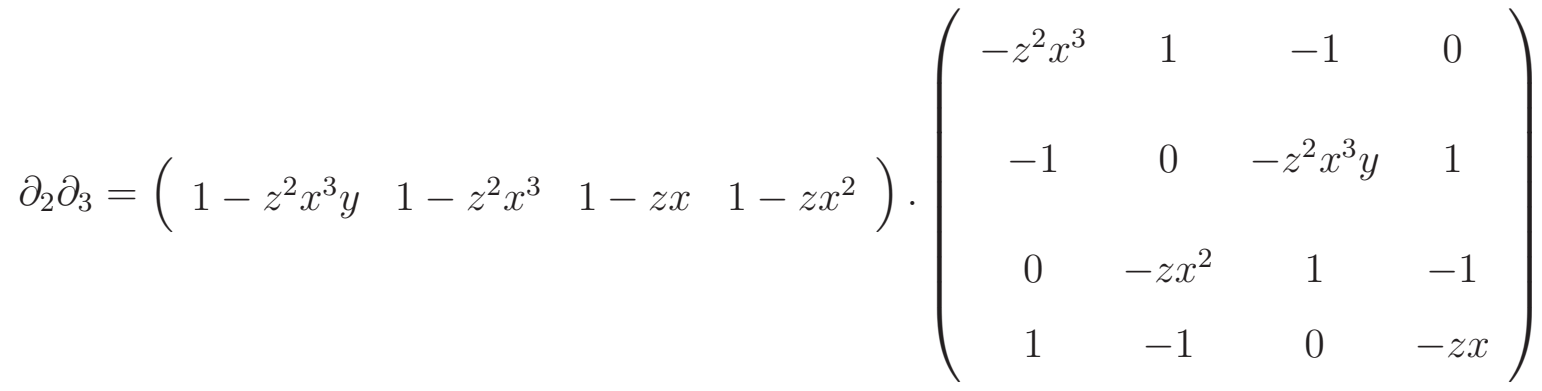

$$
\begin{aligned}
& =\left(\begin{array}{llll}
0 & 0 & 0 & 0
\end{array}\right)
\end{aligned}
$$

De fato,

- $\left(1-z^{2} x^{3} y\right)\left(-z^{2} x^{3}\right)+\left(1-z^{2} x^{3}\right)(-1)+\left(1-z x^{2}\right)=-z^{2} x^{3}+z^{2} x^{3} y z^{2} x^{3}-1+z^{2} x^{3}+1-z x^{2}$ $=z^{2} x^{3} y z^{2} x^{3}-z x^{2}=z x^{2}-z x^{2}=0$.

- $\left(1-z^{2} x^{3} y\right)+(1-z x)\left(-z x^{2}\right)+\left(1-z x^{2}\right)(-1)=1-z^{2} x^{3} y-z x^{2}+z x z x^{2}-1+z x^{2}$ $=-z^{2} x^{3} y+z x z x^{2}=-z^{2} x^{3} y+z^{2} x^{3} y=0$.

- $\left(1-z^{2} x^{3} y\right)(-1)+\left(1-z^{2} x^{3}\right)\left(-z^{2} x^{3} y\right)+(1-z x)=-1+z^{2} x^{3} y-z^{2} x^{3} y+z^{2} x^{3} z^{2} x^{3} y+1-z x$ $=z^{2} x^{3} z^{2} x^{3} y-z x=z x-z x=0$.

- $\left(1-z^{2} x^{3}\right)+(1-z x)(-1)+\left(1-z x^{2}\right)(-z x)=1-z^{2} x^{3}-1+z x-z x+z x^{2} z x=-z^{2} x^{3}+z x^{2} z x$ $=-z^{2} x^{3}+z^{2} x^{3}=0$.

A exatidão nas dimensões do meio segue do fato do complexo ser formado pelas células da decomposição celular da $S^{3}$, e da homologia da $S^{3}$ ser zero, exceto no nível zero e no nível três.

\section{$2.3 \mathrm{O}$ caso $4 n-1$ dimensional, $n>1$}

Tanto a região fundamental quanto a decomposição $C W$ para os casos de dimensões maiores seguem do caso tridimensional, visto na seção anterior, e de alguns resultados gerais, os quais serão apresentados nesta seção. 
A definição de uma região fundamental e a construção de um complexo celular equivariante de $S^{4 n-1}, n \geq 1$, são baseados no seguinte resultado geral [11, Lema 4.1], que permite reduzir a decomposição celular natural de um 'join' de esferas considerando blocos de células. Lembremos que a ação de $P_{24}$ sobre esferas de dimensão ímpar foi descrita no final da Seção 2.1.1.

Lema 2.3.1 Seja G um grupo finito agindo livremente e ortogonalmente sobre uma esfera $S^{n}$, via uma representação $\alpha$. Seja $\widetilde{K}$ uma $G$ decomposição celular de $S^{n}$, com região fundamental $\mathfrak{F}$, e dessa forma, temos uma decomposição celular $\widetilde{L}$ de $\mathfrak{F}$, onde $\widetilde{L}$ é um subcomplexo de $\widetilde{K}$, ou seja, $\widetilde{K}=G \widetilde{L}$, e $L=q(\widetilde{L})=\widetilde{L} / \alpha(G)$ é uma decomposição celular de $S^{n} / \alpha(G)$. Considere $\widetilde{Z}$ um subcomplexo de $\widetilde{L}$ que é uma decomposição minimal de $\mathfrak{F}$ formada por levantamentos das células de L. Seja $k$ um inteiro positivo com $k \leq 2$, e considere a ação natural de $G$ sobre $S^{k(n+1)-1}$, como visto na equação (2.1.3). Então, temos uma $G$ decomposição celular $\widetilde{K}^{\prime}$ de $S^{k(n+1)-1}$, com região fundamental $\mathfrak{F}^{\prime}=S^{(k-1)(n+1)-1} * \mathfrak{F}$, e uma decomposição celular minimal $\widetilde{L}^{\prime}$ de $\mathfrak{F}^{\prime}$, onde $\widetilde{L}^{\prime}$ é definida como segue: o $(k-1)(n+$ $+1)-1$ esqueleto de $\widetilde{L}^{\prime}$ é $\widetilde{L}_{((k-1)(n+1)-1)}^{\prime}=\widetilde{K}$ que é obtido juntando $k_{0}(k-1)(n+1)$ células para $\widetilde{L}_{((k-1)(n+1)-1)}^{\prime}=\widetilde{K}$, onde $k_{0}$ é o número de 0-células $\widetilde{c}_{0, l}$ de $\widetilde{Z}$ e a aplicação que faz esta junção é dada pelo 'join' $\widetilde{K} * \widetilde{c}_{0, l} ; o(k-1)(n+1)+1$ esqueleto de $\widetilde{L}^{\prime}$ é obtido juntando $k_{1}(k-1)(n+1)+1$ células para o $(k-1)(n+1)$ esqueleto $\widetilde{L}_{((k-1)(n+1))}^{\prime}$, onde $k_{1}$ é o número de 1-células $\widetilde{c}_{1, l}$ de $\widetilde{Z}$ e a aplicação que faz tal junção é dada pelo 'join' $\widetilde{L}_{((k-1)(n+1))}^{\prime} * \widetilde{c}_{1, l}$. Este processo continua até a dimensão $k(n+1)-1$, construindo assim, as decomposições celulares $\widetilde{L}^{\prime}$ de $\mathfrak{F}^{\prime}$ e $\widetilde{K}^{\prime}=G \widetilde{L}^{\prime}$ de $S^{k(n+1)-1}$.

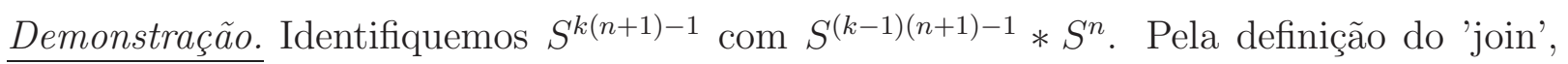
$\widetilde{B}=\widetilde{K} * \widetilde{K}$ fornece uma $G$ decomposição celular de $S^{(k-1)(n+1)-1} * S^{n}$. Como o 'join' preserva subcomplexos, $\widetilde{C}=\widetilde{K} * \widetilde{L}$ é uma decomposição celular de $\mathfrak{F}^{\prime}=S^{(k-1)(n+1)-1} * \mathfrak{F}$. Definimos agora uma nova decomposição celular $\widetilde{L}^{\prime}$ de $\mathfrak{F}^{\prime}$ reduzindo o número de células de $\widetilde{C}$, definindo novas células que são blocos das células de $\widetilde{B}$.

Primeiramente, consideremos o subcomplexo $\widetilde{L}_{((k-1)(n+1)-1)}^{\prime}=\widetilde{K} * \emptyset$ de $\widetilde{K} * \widetilde{L}$ como o $(k-1)(n+1)-1$ esqueleto de $\widetilde{L}^{\prime}$. Notemos que $\widetilde{C}$ é um $k(n+1)-1$ disco, e que o bordo de $\widetilde{C}$ é precisamente $\widetilde{K} * \partial \widetilde{L}$, já que $\widetilde{K}$ não possui bordo. Dessa forma, a ação de $G$ sobre $\widetilde{B}$ irá mandar o interior de $\widetilde{C}$ em conjuntos que interceptam somente em seus bordos, e assim, 
$\widetilde{C}$ é uma região fundamental para a ação de $G$ sobre $\widetilde{B}$.

Agora, começaremos a construção de $\widetilde{L}^{\prime}$. Seja $c_{0, j}$ uma 0-célula de $L$ e $\widetilde{c}_{0, j}$ um levantamento de $c_{0, j}$ que pertence ao interior de $\widetilde{L}$. Incluímos $\widetilde{c}_{0, j}$ em $\widetilde{C}$ como $\emptyset * \widetilde{c}_{0, j}$. Dessa forma, $\emptyset * \widetilde{c}_{0, j}$ pertence a $\widetilde{K} * \partial \widetilde{L}$, e $\widetilde{K} * \widetilde{c}_{0, j}$ são discos de dimensões $(k-1)(n+1)$, que são de fato, blocos de células de dimensões até $(k-1)(n+1)$ de $\widetilde{C}$. Observemos que, pela definição do 'join', os interiores de cada par de discos $\widetilde{K} * \widetilde{c}_{0, j}$ e $\widetilde{K} * \widetilde{c}_{0, h}$, com $h \neq j$, são disjuntos, e que a intersecção dos bordos pertencem a $\widetilde{K} * \emptyset$. Consideremos os $\operatorname{discos} \widetilde{c}_{(k-1)(n+1), j}^{\prime}=\widetilde{K} * \widetilde{c}_{0, j}$ como $k_{0}(k-1)(n+1)$ células de $\widetilde{L}^{\prime}$. Tais células são unidas ao $(k-1)(n+1)-1$ esqueleto pela definição do 'join'. Assim, o $(k-1)(n+1)$ esqueleto de $\widetilde{L}^{\prime}$ é $\widetilde{L}_{((k-1)(n+1))}^{\prime}=\bigcup \widetilde{c}_{(k-1)(n+1), j}$.

Seja $c_{1, j}$ a 1-célula de $L$ e $\widetilde{c}_{1, j}$ um levantamento de $c_{1, j}$ que pertence ao interior de $\widetilde{L}$. Incluímos $\widetilde{c}_{1, j}$ em $\widetilde{C}$ como $\emptyset * \widetilde{c}_{1, j}$. Dessa forma, $\emptyset * \widetilde{c}_{1, j}$ pertence a $\widetilde{K} * \partial \widetilde{L}$. Além disso, $\widetilde{c}_{(k-1)(n+1)+1, j}=\widetilde{K} * \widetilde{c}_{1, j}$ é um disco de dimensão $(k-1)(n+1)+1$, cujo interior está dentro de $\widetilde{K} * \partial \widetilde{L}-\widetilde{L}_{((k-1)(n+1))}^{\prime}$, enquanto o bordo pertence a $\widetilde{L}_{((k-1)(n+1))}^{\prime}$. Pela definição do 'join', os interiores de cada par de discos $\widetilde{c}_{(k-1)(n+1)+1, j}$ e $\widetilde{c}_{(k-1)(n+1)+1, k}$, com $k \neq j$, são disjuntos, e a intersecção dos bordos dos dois discos está em $\widetilde{L}_{((k-1)(n+1))}^{\prime}$. As $\widetilde{c}_{(k-1)(n+1)+1, j}$ são de fato, blocos de células de dimensões até $(k-1)(n+1)+1$ de $\widetilde{C}$. Os discos $\widetilde{c}_{(k-1)(n+1)+1, j}$ são as $k_{2}(k-1)(n+1)+1$ células de $\widetilde{L}^{\prime}$. Tais células são unidas ao $(k-1)(n+1)+1$ esqueleto de $\widetilde{L}^{\prime}$ pela definição do 'join'. O $(k-1)(n+1)+1$ esqueleto de $\widetilde{L}^{\prime}$ é $\widetilde{L}_{((k-1)(n+1))}^{\prime}=\bigcup \widetilde{c}_{(k-1)(n+1)+1, j}$.

Este processo continua até a dimensão $k(n+1)-1$, e nos dá um complexo de dimensão $k(n+1)-1$ que possui a mesma realização de $\widetilde{C}$ e assim, a nova decomposição celular desejada $\widetilde{L}^{\prime}$ de $S^{(k-1)(n+1)-1} * \mathfrak{F}$.

Como $\widetilde{c}_{q, j}$ são levantamentos de $c_{q, j}$, segue que $\widetilde{K}^{\prime}=G \widetilde{L}^{\prime}$ possui a mesma realização de $\widetilde{B}$. Isto completa a prova.

Aplicando o lema, uma região fundamental para a ação de $P_{24}$ sobre $S^{4 n-1}$ via a representação $\alpha$, segue imediatamente da definição da região fundamental $\mathfrak{F}_{24,3}$ dada na Proposição 2.2.8, ou seja: 
Proposição 2.3.2 Uma região fundamental para a ação de $P_{24}$ sobre $S^{4 n-1}, n \geq 1$, via a representação $\alpha$ é

$$
\mathfrak{F}_{24,4 n-1}=\Sigma_{1} * \Sigma_{2} * \cdots * \Sigma_{2(n-1)} * \mathfrak{F}_{24,3},
$$

com $\mathfrak{F}_{24,3}$ contida em $\Sigma_{2 n-1} * \Sigma_{2 n}$.

Também pelo Lema 2.3.1, uma decomposição celular equivariante de $S^{4 n-1}, n>1$, segue da decomposição celular de $S^{3}$ dada pela Proposição 2.2.10, do seguinte modo:

Proposição 2.3.3 Um conjunto minimal $\widetilde{Z}$ de representantes dos levantamentos $\widetilde{c}_{q, s}$ (onde o primeiro índice denota a dimensão) das células da decomposição celular $L$ de $\mathscr{P}_{4 n-1}$ é:

$$
\begin{aligned}
& \widetilde{c}_{4 q-1,1}=\Sigma^{2 q-2} * \widetilde{F}_{24,3}, \\
& \widetilde{c}_{4 q-2,1}=\Sigma^{2 q-2} * \widetilde{c}_{2,1}, \\
& \widetilde{c}_{4 q-2,2}=\Sigma^{2 q-2} * \widetilde{c}_{2,2}, \\
& \widetilde{c}_{4 q-2,3}=\Sigma^{2 q-2} * \widetilde{c}_{2,3}, \\
& \widetilde{c}_{4 q-2,4}=\Sigma^{2 q-2} * \widetilde{c}_{2,4}, \\
& \widetilde{c}_{4 q-3,1}=\Sigma^{2 q-2} * \widetilde{c}_{1,1}, \\
& \widetilde{c}_{4 q-3,2}=\Sigma^{2 q-2} * \widetilde{c}_{1,2}, \\
& \widetilde{c}_{4 q-3,3}=\Sigma^{2 q-2} * \widetilde{c}_{1,3}, \\
& \widetilde{c}_{4 q-3,4}=\Sigma^{2 q-2} * \widetilde{c}_{1,4}, \\
& \widetilde{c}_{4 q-4,1}=\Sigma^{2 q-2} * \widetilde{c}_{0,1},
\end{aligned}
$$

lembrando que $\mathfrak{F}_{24,3}, \widetilde{c}_{2,1}, \widetilde{c}_{2,2}, \widetilde{c}_{2,3}, \widetilde{c}_{2,4}, \widetilde{c}_{1,1}, \widetilde{c}_{1,2}, \widetilde{c}_{1,3}, \widetilde{c}_{1,4}$ e $\widetilde{c}_{0,1}$ estão contidas em $\Sigma_{2 q-1} * \Sigma_{2 q}$, e os operadores bordos são dados por (exceto $\partial_{0}=0$ ):

$$
\begin{aligned}
& \partial_{4 q-4}\left(\widetilde{c}_{4 q-4,1}\right)=\left(1+x+x^{2}+x^{3}\right)(1+y)\left(1+z+z^{2}\right) \widetilde{c}_{(4 q-4)-1,1}, \\
& \partial_{4 q-3}\left(\widetilde{c}_{4 q-3,1}\right)=\left(z^{2} x^{3} y-1\right) \widetilde{c}_{4 q-4,1}, \\
& \partial_{4 q-3}\left(\widetilde{c}_{4 q-3,2}\right)=(z x-1) \widetilde{c}_{4 q-4,1}, \\
& \partial_{4 q-3}\left(\widetilde{c}_{4 q-3,3}\right)=\left(z^{2} x^{3}-1\right) \widetilde{c}_{4 q-4,1}, \\
& \partial_{4 q-3}\left(\widetilde{c}_{4 q-3,4}\right)=\left(z x^{2}-1\right) \widetilde{c}_{4 q-4,1}, \\
& \partial_{4 q-2}\left(\widetilde{c}_{4 q-2,1}\right)=-z^{2} x^{3} \widetilde{c}_{4 q-3,1}+\widetilde{c}_{4 q-3,2}-\widetilde{c}_{4 q-3,3}, \\
& \partial_{4 q-2}\left(\widetilde{c}_{4 q-2,2}\right)=-\widetilde{c}_{4 q-3,1}-z^{2} x^{3} y \widetilde{c}_{4 q-3,3}+\widetilde{c}_{4 q-3,4},
\end{aligned}
$$




$$
\begin{aligned}
& \partial_{4 q-2}\left(\widetilde{c}_{4 q-2,3}\right)=-z x^{2} \widetilde{c}_{4 q-3,2}+\widetilde{c}_{4 q-3,3}-\widetilde{c}_{4 q-3,4}, \\
& \partial_{4 q-2}\left(\widetilde{c}_{4 q-2,4}\right)=\widetilde{c}_{4 q-3,1}-\widetilde{c}_{4 q-3,2}-z x \widetilde{c}_{4 q-3,4} \\
& \partial_{4 q-1}\left(\widetilde{c}_{4 q-1,1}\right)=\left(1-z^{2} x^{3} y\right) \widetilde{c}_{4 q-2,1}+\left(1-z^{2} x^{3}\right) \widetilde{c}_{4 q-2,2}+(1-z x) \widetilde{c}_{4 q-2,3}+\left(1-z x^{2}\right) \widetilde{c}_{4 q-2,4} .
\end{aligned}
$$

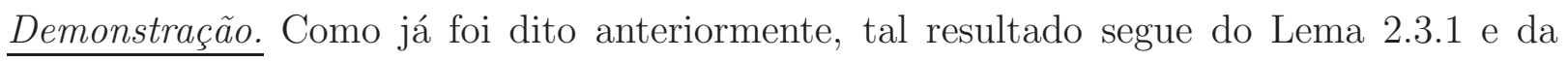
decomposição celular equivariante de $S^{3}$ dada pela Proposição 2.2.10. O único ponto que precisa ser completado é o operador bordo da célula $\widetilde{c}_{4 q-4,1}, q>0$. Porém, ele segue facilmente considerando que $\widetilde{c}_{4 q-4,1}=S^{(4 q-4)-1} * \widetilde{c}_{(4 q-4)-1,1}$, e assim seu bordo é dado pela coleção de todas as células de $S^{(4 q-4)-1}$, isto é, todas as órbitas de $\alpha$.

Agora, usando a decomposição celular e os operadores bordos das células descritas na proposição anterior, obtemos o seguinte resultado para o espaço $\mathscr{P}_{4 n-1}, n \geq 1$ :

Teorema 2.3.4 $O$ complexo de cadeias $C\left(\mathscr{P}_{4 n-1} ; \mathbb{Z} P_{24}\right)$ do espaço de recobrimento universal das formas espaciais esféricas tetraedrais, com a ação do grupo fundamental atuando por transformações de recobrimento, é o seguinte complexo de $\mathbb{Z} P_{24}$ módulos livres e finitamente gerados:

$$
\cdots \longrightarrow C_{4 q-1} \stackrel{\partial_{4 q-1}}{\longrightarrow} C_{4 q-2} \stackrel{\partial_{4 q-2}}{\longrightarrow} C_{4 q-3} \stackrel{\partial_{4 q-3}}{\longrightarrow} C_{4 q-4} \stackrel{\partial_{4 q-4}}{\longrightarrow} \cdots
$$

onde

$$
\begin{aligned}
& C_{4 q-1}=\mathbb{Z} P_{24}\left[\widetilde{c}_{4 q-1,1}\right], \\
& C_{4 q-2}=\mathbb{Z} P_{24}\left[\widetilde{c}_{4 q-2,1}, \widetilde{c}_{4 q-2,2}, \widetilde{c}_{4 q-2,3}, \widetilde{c}_{4 q-2,4}\right], \\
& C_{4 q-3}=\mathbb{Z} P_{24}\left[\widetilde{c}_{4 q-3,1}, \widetilde{c}_{4 q-3,2}, \widetilde{c}_{4 q-3,3}, \widetilde{c}_{4 q-3,4}\right], \\
& C_{4 q-4}=\mathbb{Z} P_{24}\left[\widetilde{c}_{4 q-4,1}\right],
\end{aligned}
$$

com os operadores bordos dados como no enunciado da Proposição 2.3.3. Tal complexo é exato em todas as dimensões do meio.

Demonstração. O complexo de cadeias segue diretamente da decomposição celular de $S^{4 n-1}$ descrita na Proposição 2.3.3, vista anteriormente. 
Afirmação 2.3.1 O complexo de cadeias do teorema é semi-exato.

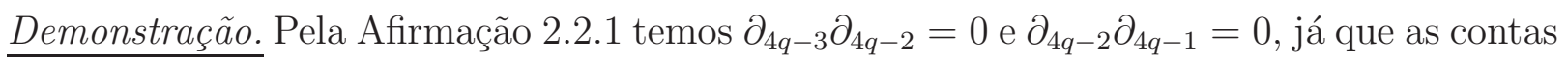
são análogas a prova de que $\partial_{1} \partial_{2}=0$ e que $\partial_{2} \partial_{3}=0$, respectivamente.

Resta mostrar que $\partial_{4 q-1} \partial_{4 q-4}=0$ e $\partial_{4 q-4} \partial_{4 q-3}=0$.

Temos,

$$
\partial_{4 q-1} \partial_{4 q-4}=\left(\begin{array}{llll}
0 & 0 & 0 & 0
\end{array}\right)
$$

De fato,

$$
\begin{gathered}
\partial_{4 q-4}=\left(\left(1+x+x^{2}+x^{3}\right)(1+y)\left(1+z+z^{2}\right)\right)=1+x+x^{2}+x^{3}+y+x y+x^{2} y+x^{3} y+z+ \\
+z x+z x^{2}+z x^{3}+z y+z x y+z x^{2} y+z x^{3} y+z^{2}+z^{2} x+z^{2} x^{2}+z^{2} x^{3}+z^{2} y+z^{2} x y+z^{2} x^{2} y+z^{2} x^{3} y .
\end{gathered}
$$

Isto é, a multiplicação acima resulta na soma de todos os elementos de $P_{24}$. Assim,

$$
\left(\left(1+x+x^{2}+x^{3}\right)(1+y)\left(1+z+z^{2}\right)\right) \cdot\left(1-z^{2} x^{3} y\right)=0
$$

uma vez que, ao multiplicarmos $\partial_{4 q-4}$ por 1 , teremos todos os elementos de $P_{24}$ com sinal positivo e ao multiplicarmos $\partial_{4 q-4}$ por $-z^{2} x^{3} y$, teremos todos os elementos de $P_{24}$ com sinal negativo, ou seja, a soma desses elementos será zero.

Analogamente,

$$
\begin{aligned}
& \left(\left(1+x+x^{2}+x^{3}\right)(1+y)\left(1+z+z^{2}\right)\right) \cdot\left(1-z^{2} x^{3}\right)=0 \\
& \left(\left(1+x+x^{2}+x^{3}\right)(1+y)\left(1+z+z^{2}\right)\right) \cdot(1-z x)=0
\end{aligned}
$$

e

$$
\left(\left(1+x+x^{2}+x^{3}\right)(1+y)\left(1+z+z^{2}\right)\right) \cdot\left(1-z x^{2}\right)=0
$$

Agora,

$$
\partial_{4 q-4} \partial_{4 q-3}=\left(\begin{array}{c}
0 \\
0 \\
0 \\
0
\end{array}\right)
$$

De fato, 


$$
\begin{gathered}
\partial_{4 q-4}=\left(\left(1+x+x^{2}+x^{3}\right)(1+y)\left(1+z+z^{2}\right)\right)=1+x+x^{2}+x^{3}+y+x y+x^{2} y+x^{3} y+z+ \\
+z x+z x^{2}+z x^{3}+z y+z x y+z x^{2} y+z x^{3} y+z^{2}+z^{2} x+z^{2} x^{2}+z^{2} x^{3}+z^{2} y+z^{2} x y+z^{2} x^{2} y+z^{2} x^{3} y .
\end{gathered}
$$

Ou seja, a multiplicação acima resulta na soma de todos os elementos de $P_{24}$. Logo,

$$
\left(z^{2} x^{3} y-1\right) \cdot\left(\left(1+x+x^{2}+x^{3}\right)(1+y)\left(1+z+z^{2}\right)\right)=0
$$

pois, ao multiplicarmos $z^{2} x^{3} y$ por $\partial_{4 q-4}$, teremos todos os elementos de $P_{24}$ com sinal positivo e ao multiplicarmos -1 por $\partial_{4 q-4}$, teremos todos os elementos de $P_{24}$ com sinal negativo, ou seja, a soma desses elementos será zero.

Analogamente,

$$
\begin{aligned}
& (z x-1) \cdot\left(\left(1+x+x^{2}+x^{3}\right)(1+y)\left(1+z+z^{2}\right)\right)=0 \\
& \left(z^{2} x^{3}-1\right) \cdot\left(\left(1+x+x^{2}+x^{3}\right)(1+y)\left(1+z+z^{2}\right)\right)=0
\end{aligned}
$$

e

$$
\left(z x^{2}-1\right) \cdot\left(\left(1+x+x^{2}+x^{3}\right)(1+y)\left(1+z+z^{2}\right)\right)=0 .
$$

A exatidão nas dimensões do meio segue, como no caso tridimensional, do fato do complexo ser formado pelas células da decomposição celular da $S^{4 n-1}$, e da homologia da $S^{4 n-1}$ ser zero nos níveis do meio.

Como o complexo de cadeias $C\left(\mathscr{P}_{4 n-1} ; \mathbb{Z} P_{24}\right)$ do espaço de recobrimento $\left(S^{4 n-1}\right)$ é exato em todas as dimensões do meio, isto é, $\operatorname{Im}\left(\partial_{j+1}\right)=\operatorname{Ker}\left(\partial_{j}\right), 0<j<4 n-1$, resta apenas verificar o nível $4 n-1$. Utilizando o homomorfismo conectante e a aplicação aumentação, construímos uma 4-periódica resolução livre de $\mathbb{Z}$ sobre $\mathbb{Z} P_{24}$, como mostra o resultado a seguir.

Corolário 2.3.5 O complexo

$$
\cdots \longrightarrow C_{4 q-1} \stackrel{\partial_{4 q-1}}{\longrightarrow} C_{4 q-2} \stackrel{\partial_{4 q-2}}{\longrightarrow} C_{4 q-3} \stackrel{\partial_{4 q-3}}{\longrightarrow} C_{4 q-4} \stackrel{\partial_{4 q-4}}{\longrightarrow} \cdots \longrightarrow C_{1} \stackrel{\partial_{1}}{\longrightarrow} C_{0} \stackrel{\varepsilon}{\longrightarrow} \mathbb{Z} \longrightarrow 0
$$


é uma 4-periódica resolução livre de $\mathbb{Z}$ sobre $\mathbb{Z} P_{24}$.

Apresentaremos agora, um exemplo envolvendo a $S^{7}$ para um melhor entendimento desta seção.

Exemplo 2.3.6 Considere a figura abaixo:
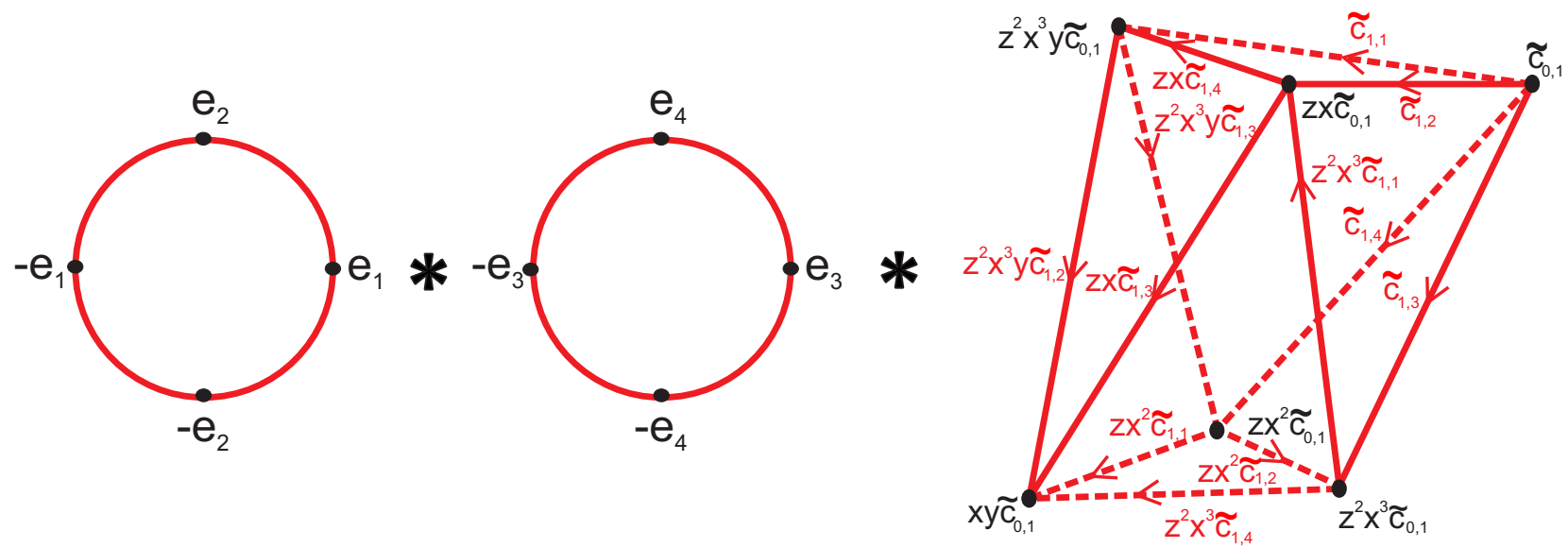

Notemos que $\Sigma_{1} * \Sigma_{2}$ é uma 3-esfera.

A região fundamental da ação de $P_{24}$ sobre $S^{7}$ é $\mathfrak{F}_{24,7}=\Sigma_{1} * \Sigma_{2} * \mathfrak{F}_{24,3}$.

As células fundamentais são:

$$
\begin{gathered}
\widetilde{c}_{7,1}=\Sigma_{1} * \Sigma_{2} * \widetilde{F}_{24,3}, \\
\widetilde{c}_{6,1}=\Sigma_{1} * \Sigma_{2} * \widetilde{c}_{2,1}, \\
\widetilde{c}_{6,2}=\Sigma_{1} * \Sigma_{2} * \widetilde{c}_{2,2}, \\
\widetilde{c}_{6,3}=\Sigma_{1} * \Sigma_{2} * \widetilde{c}_{2,3}, \\
\widetilde{c}_{6,4}=\Sigma_{1} * \Sigma_{2} * \widetilde{c}_{2,4}, \\
\widetilde{c}_{5,1}=\Sigma_{1} * \Sigma_{2} * \widetilde{c}_{1,1}, \\
\widetilde{c}_{5,2}=\Sigma_{1} * \Sigma_{2} * \widetilde{c}_{1,2}, \\
\widetilde{c}_{5,3}=\Sigma_{1} * \Sigma_{2} * \widetilde{c}_{1,3}, \\
\widetilde{c}_{5,4}=\Sigma_{1} * \Sigma_{2} * \widetilde{c}_{1,4}, \\
\widetilde{c}_{4,1}=\Sigma_{1} * \Sigma_{2} * \widetilde{c}_{0,1}, \\
\widetilde{c}_{3,1}=\mathfrak{F}_{24,3}, \\
\widetilde{c}_{2,1}=\widetilde{c}_{2,1},
\end{gathered}
$$




$$
\begin{aligned}
& \widetilde{c}_{2,2}=\widetilde{c}_{2,2}, \\
& \widetilde{c}_{2,3}=\widetilde{c}_{2,3}, \\
& \widetilde{c}_{2,4}=\widetilde{c}_{2,4}, \\
& \widetilde{c}_{1,1}=\widetilde{c}_{1,1}, \\
& \widetilde{c}_{1,2}=\widetilde{c}_{1,2}, \\
& \widetilde{c}_{1,3}=\widetilde{c}_{1,3}, \\
& \widetilde{c}_{1,4}=\widetilde{c}_{1,4}, \\
& \widetilde{c}_{0,1}=\widetilde{c}_{0,1} .
\end{aligned}
$$

E os operadores bordos são dados por

$$
\begin{gathered}
\partial_{7}=\partial_{3}=\left(\begin{array}{cccc}
1-z^{2} x^{3} y & 1-z^{2} x^{3} & 1-z x & 1-z x^{2}
\end{array}\right) \\
\partial_{6}=\partial_{2}=\left(\begin{array}{cccc}
-z^{2} x^{3} & 1 & -1 & 0 \\
-1 & 0 & -z^{2} x^{3} y & 1 \\
0 & -z x^{2} & 1 & -1 \\
1 & -1 & 0 & -z x
\end{array}\right) \\
\partial_{5}=\partial_{1}=\left(\begin{array}{c}
z^{2} x^{3} y-1 \\
z x-1 \\
z^{2} x^{3}-1 \\
z x^{2}-1
\end{array}\right)
\end{gathered}
$$

$e$

$$
\partial_{4}=\left(\left(1+x+x^{2}+x^{3}\right)(1+y)\left(1+z+z^{2}\right)\right)
$$

Obtemos o seguinte complexo de cadeias sobre o anel $\mathbb{Z} P_{24}$, possuindo apenas uma 0-célula, quatro 1-células, quatro 2-células, uma 3-célula, uma 4-célula, quatro 5-células, quatro 6-células e uma \%-célula.

$$
0 \longrightarrow \mathbb{Z} P_{24}\left[\widetilde{c}_{7,1}\right] \stackrel{\partial_{7}}{\longrightarrow} \mathbb{Z} P_{24}\left[\widetilde{c}_{6,1}, \widetilde{c}_{6,2}, \widetilde{c}_{6,3}, \widetilde{c}_{6,4}\right] \stackrel{\partial_{6}}{\longrightarrow} \mathbb{Z} P_{24}\left[\widetilde{c}_{5,1}, \widetilde{c}_{5,2}, \widetilde{c}_{5,3}, \widetilde{c}_{5,4}\right] \stackrel{\partial_{5}}{\longrightarrow} \mathbb{Z} P_{24}\left[\widetilde{c}_{4,1}\right] \stackrel{\partial_{4}}{\longrightarrow}
$$


$\stackrel{\partial_{4}}{\longrightarrow} \mathbb{Z} P_{24}\left[\widetilde{c}_{3,1}\right] \stackrel{\partial_{3}}{\longrightarrow} \mathbb{Z} P_{24}\left[\widetilde{c}_{2,1}, \widetilde{c}_{2,2}, \widetilde{c}_{2,3}, \widetilde{c}_{2,4}\right] \stackrel{\partial_{2}}{\longrightarrow} \mathbb{Z} P_{24}\left[\widetilde{c}_{1,1}, \widetilde{c}_{1,2}, \widetilde{c}_{1,3}, \widetilde{c}_{1,4}\right] \stackrel{\partial_{1}}{\longrightarrow} \mathbb{Z} P_{24}\left[\widetilde{c}_{0,1}\right] \longrightarrow 0$.

Afrmação. O complexo de cadeias anterior é semi-exato.

Demonstração. Temos,

$$
\partial_{5} \partial_{6}=\partial_{1} \partial_{2}=\left(\begin{array}{cccc}
-z^{2} x^{3} & 1 & -1 & 0 \\
-1 & 0 & -z^{2} x^{3} y & 1 \\
0 & -z x^{2} & 1 & -1 \\
1 & -1 & 0 & -z x
\end{array}\right) \cdot\left(\begin{array}{c}
z^{2} x^{3} y-1 \\
z x-1 \\
z^{2} x^{3}-1 \\
z x^{2}-1
\end{array}\right)=\left(\begin{array}{l}
0 \\
0 \\
0 \\
0
\end{array}\right)
$$

De fato,

- $-z^{2} x^{3}\left(z^{2} x^{3} y-1\right)+(z x-1)-\left(z^{2} x^{3}-1\right)=-z^{2} x^{3} z^{2} x^{3} y+z^{2} x^{3}+z x-1-z^{2} x^{3}+1$ $=-z^{2} x^{2} y z y z x^{3} y+z x=-z^{2} z x y y^{2} z x^{3} y+z x=-x y z x^{2} x^{3} y+z x=-z y x y+z x=-z x+z x$ $=0$.

- $-\left(z^{2} x^{3} y-1\right)-z^{2} x^{3} y\left(z^{2} x^{3}-1\right)+\left(z x^{2}-1\right)=-z^{2} x^{3} y+1-z^{2} x^{3} y z^{2} x^{3}+z^{2} x^{3} y+z x^{2}-1$

$=-z^{2} x y y z y z y x^{3}+z x^{2}=-z^{2} x y z x y z x^{3} y^{3}+z x^{2}=-z^{2} z y x y z x y+z x^{2}=-y z y x y+z x^{2}$ $=-z x^{2}+z x^{2}=0$.

- $-z x^{2}(z x-1)+\left(z^{2} x^{3}-1\right)-\left(z x^{2}-1\right)=-z x^{2} z x+z x^{2}+z^{2} x^{3}-1-z x^{2}+1=-z z x^{2} x+z^{2} x^{3}$ $=-z^{2} x^{3}+z^{2} x^{3}=0$.

- $\left(z^{2} x^{3} y-1\right)-(z x-1)-z x\left(z x^{2}-1\right)=z^{2} x^{3} y-1-z x+1-z x z x^{2}+z x=z^{2} x^{3} y-z z x y x^{2}$ $=z^{2} x^{3} y-z^{2} x x^{2} y=z^{2} x^{3} y-z^{2} x^{3} y=0$.

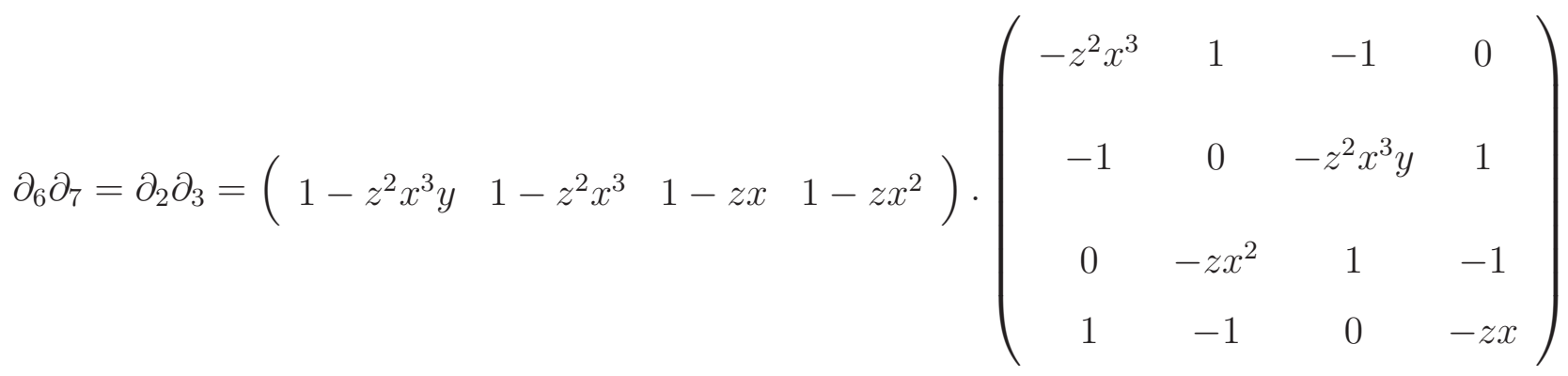

$$
\begin{aligned}
& =\left(\begin{array}{llll}
0 & 0 & 0 & 0
\end{array}\right)
\end{aligned}
$$


De fato,

- $\left(1-z^{2} x^{3} y\right)\left(-z^{2} x^{3}\right)+\left(1-z^{2} x^{3}\right)(-1)+\left(1-z x^{2}\right)=-z^{2} x^{3}+z^{2} x^{3} y z^{2} x^{3}-1+z^{2} x^{3}+1-z x^{2}$ $=z^{2} x^{3} y z^{2} x^{3}-z x^{2}=z x^{2}-z x^{2}=0$.

- $\left(1-z^{2} x^{3} y\right)+(1-z x)\left(-z x^{2}\right)+\left(1-z x^{2}\right)(-1)=1-z^{2} x^{3} y-z x^{2}+z x z x^{2}-1+z x^{2}$ $=-z^{2} x^{3} y+z x z x^{2}=-z^{2} x^{3} y+z^{2} x^{3} y=0$.

- $\left(1-z^{2} x^{3} y\right)(-1)+\left(1-z^{2} x^{3}\right)\left(-z^{2} x^{3} y\right)+(1-z x)=-1+z^{2} x^{3} y-z^{2} x^{3} y+z^{2} x^{3} z^{2} x^{3} y+1-z x$ $=z^{2} x^{3} z^{2} x^{3} y-z x=z x-z x=0$.

- $\left(1-z^{2} x^{3}\right)+(1-z x)(-1)+\left(1-z x^{2}\right)(-z x)=1-z^{2} x^{3}-1+z x-z x+z x^{2} z x=-z^{2} x^{3}+z x^{2} z x$ $=-z^{2} x^{3}+z^{2} x^{3}=0$.

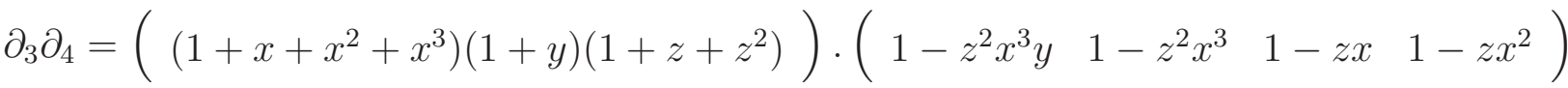

$$
\begin{aligned}
& =\left(\begin{array}{llll}
0 & 0 & 0 & 0
\end{array}\right)
\end{aligned}
$$

De fato,

- $\left(1+x+x^{2}+x^{3}+y+x y+x^{2} y+x^{3} y+z+z x+z x^{2}+z x^{3}+z y+z x y+z x^{2} y+z x^{3} y+\right.$ $\left.+z^{2}+z^{2} x+z^{2} x^{2}+z^{2} x^{3}+z^{2} y+z^{2} x y+z^{2} x^{2} y+z^{2} x^{3} y\right)\left(1-z^{2} x^{3} y\right)=1+x+x^{2}+x^{3}+y+$ $+x y+x^{2} y+x^{3} y+z+z x+z x^{2}+z x^{3}+z y+z x y+z x^{2} y+z x^{3} y+z^{2}+z^{2} x+z^{2} x^{2}+z^{2} x^{3}+$ $+z^{2} y+z^{2} x y+z^{2} x^{2} y+z^{2} x^{3} y-z^{2} x^{3} y-z^{2} x^{3}-z^{2} x y-z^{2} x-z^{2}-z^{2} y-z^{2} x^{2}-z^{2} x^{2} y-x^{3} y-$ $-x^{3}-x y-x-1-y-x^{2}-x^{2} y-z x^{3} y-z x^{3}-z x y-z x-z-z y-z x^{2}-z x^{2} y=0$.

- $\left(1+x+x^{2}+x^{3}+y+x y+x^{2} y+x^{3} y+z+z x+z x^{2}+z x^{3}+z y+z x y+z x^{2} y+z x^{3} y+\right.$ $\left.+z^{2}+z^{2} x+z^{2} x^{2}+z^{2} x^{3}+z^{2} y+z^{2} x y+z^{2} x^{2} y+z^{2} x^{3} y\right)\left(1-z^{2} x^{3}\right)=1+x+x^{2}+x^{3}+y+$ $+x y+x^{2} y+x^{3} y+z+z x+z x^{2}+z x^{3}+z y+z x y+z x^{2} y+z x^{3} y+z^{2}+z^{2} x+z^{2} x^{2}+z^{2} x^{3}+$ $+z^{2} y+z^{2} x y+z^{2} x^{2} y+z^{2} x^{3} y-z^{2} x^{3}-z^{2} x y-z^{2} x-z^{2} x^{3} y-z^{2} x^{2} y-z^{2}-z^{2} y-z^{2} x^{2}-x^{3}-$ $-x y-x-x^{3} y-x^{2} y-1-y-x^{2}-z x^{3}-z x y-z x-z x^{3} y-z x^{2} y-z-z y-z x^{2}=0$.

- $\left(1+x+x^{2}+x^{3}+y+x y+x^{2} y+x^{3} y+z+z x+z x^{2}+z x^{3}+z y+z x y+z x^{2} y+z x^{3} y+\right.$ $\left.+z^{2}+z^{2} x+z^{2} x^{2}+z^{2} x^{3}+z^{2} y+z^{2} x y+z^{2} x^{2} y+z^{2} x^{3} y\right)(1-z x)=1+x+x^{2}+x^{3}+y+x y+$ $+x^{2} y+x^{3} y+z+z x+z x^{2}+z x^{3}+z y+z x y+z x^{2} y+z x^{3} y+z^{2}+z^{2} x+z^{2} x^{2}+z^{2} x^{3}+z^{2} y+$ 
$+z^{2} x y+z^{2} x^{2} y+z^{2} x^{3} y-z x-z y-z x^{3}-z x^{2} y-z x^{2}-z x^{3} y-z-z x y-z^{2} x-z^{2} y-z^{2} x^{3}-$ $-z^{2} x^{2} y-z^{2} x^{2}-z^{2} x^{3} y-z^{2}-z^{2} x y-x-y-x^{3}-x^{2} y-x^{2}-x^{3} y-1-x y=0$.

- $\left(1+x+x^{2}+x^{3}+y+x y+x^{2} y+x^{3} y+z+z x+z x^{2}+z x^{3}+z y+z x y+z x^{2} y+z x^{3} y+\right.$ $\left.+z^{2}+z^{2} x+z^{2} x^{2}+z^{2} x^{3}+z^{2} y+z^{2} x y+z^{2} x^{2} y+z^{2} x^{3} y\right)\left(1-z x^{2}\right)=1+x+x^{2}+x^{3}+y+x y+$ $+x^{2} y+x^{3} y+z+z x+z x^{2}+z x^{3}+z y+z x y+z x^{2} y+z x^{3} y+z^{2}+z^{2} x+z^{2} x^{2}+z^{2} x^{3}+z^{2} y+$ $+z^{2} x y+z^{2} x^{2} y+z^{2} x^{3} y-z x^{2}-z x^{3} y-z-z x y-z x^{3}-z x^{2} y-z x-z y-z^{2} x^{2}-z^{2} x^{3} y-z^{2}-$ $-z^{2} x y-z^{2} x^{3}-z^{2} x^{2} y-z^{2} x-z^{2} y-x^{2}-x^{3} y-1-x y-x^{3}-x^{2} y-x-y=0$.

$$
\partial_{4} \partial_{5}=\left(\begin{array}{c}
z^{2} x^{3} y-1 \\
z x-1 \\
z^{2} x^{3}-1 \\
z x^{2}-1
\end{array}\right) \cdot\left(\left(1+x+x^{2}+x^{3}\right)(1+y)\left(1+z+z^{2}\right)\right)=\left(\begin{array}{l}
0 \\
0 \\
0 \\
0
\end{array}\right)
$$

De fato,

- $\left(z^{2} x^{3} y-1\right)\left(1+x+x^{2}+x^{3}+y+x y+x^{2} y+x^{3} y+z+z x+z x^{2}+z x^{3}+z y+z x y+z x^{2} y+\right.$ $\left.+z x^{3} y+z^{2}+z^{2} x+z^{2} x^{2}+z^{2} x^{3}+z^{2} y+z^{2} x y+z^{2} x^{2} y+z^{2} x^{3} y\right)=z^{2} x^{3} y+z^{2} x^{2} y+z^{2} x y+z^{2} y+$ $+z^{2} x+z^{2}+z^{2} x^{3}+z^{2} x^{2}+x^{2} y+x y+y+x^{3} y+1+x^{3}+x^{2}+x+z x^{3}+z+z x+z x^{2}+z x^{3} y+$ $+z y+z x y+z x^{2} y-1-x-x^{2}-x^{3}-y-x y-x^{2} y-x^{3} y-z-z x-z x^{2}-z x^{3}-z y-z x y-$ $-z x^{2} y-z x^{3} y-z^{2}-z^{2} x-z^{2} x^{2}-z^{2} x^{3}-z^{2} y-z^{2} x y-z^{2} x^{2} y-z^{2} x^{3} y=0$.

- $(z x-1)\left(1+x+x^{2}+x^{3}+y+x y+x^{2} y+x^{3} y+z+z x+z x^{2}+z x^{3}+z y+z x y+z x^{2} y+\right.$ $\left.+z x^{3} y+z^{2}+z^{2} x+z^{2} x^{2}+z^{2} x^{3}+z^{2} y+z^{2} x y+z^{2} x^{2} y+z^{2} x^{3} y\right)=z x+z x^{2}+z x^{3}+z+z x y+z x^{2} y+$ $+z x^{3} y+z y+z^{2} x y+z^{2} y+z^{2} x^{3} y+z^{2} x^{2} y+z^{2} x^{3}+z^{2} x^{2}+z^{2} x+z^{2}+y+x^{3} y+x^{2} y+$ $+x y+x^{2}+x+1+x^{3}-1-x-x^{2}-x^{3}-y-x y-x^{2} y-x^{3} y-z-z x-z x^{2}-z x^{3}-z y-$ $-z x y-z x^{2} y-z x^{3} y-z^{2}-z^{2} x-z^{2} x^{2}-z^{2} x^{3}-z^{2} y-z^{2} x y-z^{2} x^{2} y-z^{2} x^{3} y=0$.

- $\left(z^{2} x^{3}-1\right)\left(1+x+x^{2}+x^{3}+y+x y+x^{2} y+x^{3} y+z+z x+z x^{2}+z x^{3}+z y+z x y+z x^{2} y+\right.$ $\left.+z x^{3} y+z^{2}+z^{2} x+z^{2} x^{2}+z^{2} x^{3}+z^{2} y+z^{2} x y+z^{2} x^{2} y+z^{2} x^{3} y\right)=z^{2} x^{3}+z^{2}+z^{2} x+z^{2} x^{2}+$ $+z^{2} x^{3} y+z^{2} y+z^{2} x y+z^{2} x^{2} y+x^{3} y+x^{2} y+x y+y+x+1+x^{3}+x^{2}+z x^{2} y+z x y+z y+$ $+z x^{3} y+z+z x^{3}+z x^{2}+z x-1-x-x^{2}-x^{3}-y-x y-x^{2} y-x^{3} y-z-z x-z x^{2}-z x^{3}-$ 
$-z y-z x y-z x^{2} y-z x^{3} y-z^{2}-z^{2} x-z^{2} x^{2}-z^{2} x^{3}-z^{2} y-z^{2} x y-z^{2} x^{2} y-z^{2} x^{3} y=0$.

- $\left(z x^{2}-1\right)\left(1+x+x^{2}+x^{3}+y+x y+x^{2} y+x^{3} y+z+z x+z x^{2}+z x^{3}+z y+z x y+z x^{2} y+\right.$ $\left.+z x^{3} y+z^{2}+z^{2} x+z^{2} x^{2}+z^{2} x^{3}+z^{2} y+z^{2} x y+z^{2} x^{2} y+z^{2} x^{3} y\right)=z x^{2}+z x^{3}+z+z x+z x^{2} y+$ $+z x^{3} y+z y+z x y+z^{2} x^{2}+z^{2} x^{3}+z^{2}+z^{2} x+z^{2} x^{2} y+z^{2} x^{3} y+z^{2} y+z^{2} x y+x^{2}+x^{3}+1+x+$ $+x^{2} y+x^{3} y+y+x y-1-x-x^{2}-x^{3}-y-x y-x^{2} y-x^{3} y-z-z x-z x^{2}-z x^{3}-z y-$ $-z x y-z x^{2} y-z x^{3} y-z^{2}-z^{2} x-z^{2} x^{2}-z^{2} x^{3}-z^{2} y-z^{2} x y-z^{2} x^{2} y-z^{2} x^{3} y=0$. 
Capítulo

3

\section{Grupos de homologia das formas espaciais esféricas tetraedrais}

Neste capítulo, utilizamos o complexo de cadeias construído no capítulo anterior para calcular os grupos de homologia (com coeficientes locais) das formas espaciais esféricas tetraedrais com coeficientes em $\mathbb{Z}, \mathbb{Z}_{2}, \mathbb{Z}_{3}$ e $\mathbb{Z}_{p}$, com $p$ primo e $p>3$. Tais grupo serão necessários para o cálculo dos grupos de cohomologia (com coeficientes locais) e do anel de cohomologia de tais variedades, que serão apresentados no capítulo a seguir. A referência principal para este capítulo é [8].

\subsection{Homologia com coeficientes locais}

No caso de nosso interesse, o complexo de cadeias que será associado a um espaço $X$ é um complexo celular da cobertura universal $\tilde{X}$, visto como um complexo de cadeias sobre o anel de grupos $\mathbb{Z}\left[\pi_{1}(X)\right]$. Desse ponto de vista, coeficientes locais nada mais são que módulos sobre o anel de grupos $\mathbb{Z}\left[\pi_{1}(X)\right]$.

Neste capítulo, trabalharemos com $\mathbb{Z}$-módulos (módulos sobre o anel $\mathbb{Z}$ ) e módulos sobre o anel de grupos $\mathbb{Z} G$. Se $G$ não é abeliano, o anel $\mathbb{Z} G$ não é necessariamente comutativo e assim devemos distinguir entre módulos à direita e módulos à esquerda. 
Seja $A$ um grupo abeliano e seja

$$
\eta: G \rightarrow A u t_{\mathbb{Z}}(A)
$$

um homomorfismo. Neste caso dizemos que $\eta$ ou $A$ é uma representação de $G$. A representação $\eta$ induz em $A$ uma estrutura de $\mathbb{Z} G$-módulo à esquerda, onde a ação é dada por

$$
\left(\sum_{g \in G} m_{g} g\right) \cdot a=\sum_{g \in G} m_{g} \eta(g)(a) .
$$

Reciprocamente, se $A$ é um módulo à esquerda sobre o anel de grupos $\mathbb{Z} G$, existe um homomorfismo

$$
\eta: G \rightarrow A u t_{\mathbb{Z}}(A)
$$

dado por $g \mapsto(a \mapsto g a)$, onde $g a$ é a multiplicação de $a \in A$ por $g \in \mathbb{Z} G$. Assim, uma representação de um grupo $G$ sobre um grupo abeliano é o mesmo que um $\mathbb{Z} G$-módulo.

O primeiro ponto na construção da homologia com coeficientes locais é observarmos que o complexo de cadeias celular da cobertura universal de um espaço é um $\mathbb{Z} G$-módulo à direita, ou seja, $\mathbb{Z} G$ age em tal complexo.

Para isso, seja $X$ um espaço conexo por caminhos e localmente conexo por caminhos, com ponto base escolhido, que admite uma cobertura universal. Para facilidade de notação, seja $\pi=\pi_{1}(X)$. Seja $\widetilde{X} \rightarrow X$ a cobertura universal de $X$, com a ação à direita usual de $\pi$. Então o complexo celular $C(\widetilde{X})$ da cobertura universal (com coeficientes inteiros) é um $\mathbb{Z} G$-módulo à direita.

Podemos agora introduzir a definição de homologia com coeficientes locais.

Definição 3.1.1 Dado um $\mathbb{Z} G$-módulo A, formamos o produto tensorial

$$
C(X ; A)=C(\widetilde{X}) \otimes_{\mathbb{Z} G} A
$$

Este é um complexo de cadeias cuja homologia é chamada homologia de X com coeficientes locais em $A$, denotada por $H_{*}(X ; A)$.

Notemos que, desde que o anel $\mathbb{Z} G$ é não-comutativo (exceto se $G$ for abeliano), o complexo de cadeias tensorizado tem somente a estrutura de um complexo de cadeias sobre 
$\mathbb{Z}$, não sobre $\mathbb{Z} G$. Assim o grupo de homologia $H_{*}(X ; A)$ é somente um $\mathbb{Z}$-módulo. Tal informação será importante para justificar alguns fatos que ocorrerão durante o cálculo das homologias.

Se o $\mathbb{Z} G$-módulo $A$ é especificado por $\eta: \pi_{1}(X) \rightarrow A u t(A)$, e se quisermos enfatizar tal representação, escreveremos então $H_{*}\left(X ; A_{\eta}\right)$. As vezes, é comum chamarmos $H_{*}\left(X, A_{\eta}\right)$ de homologia de $X$ torcida por $\eta$.

\subsection{Grupos de homologia de $\mathscr{P}_{3}$}

Vamos calcular os grupos de homologia de $\mathscr{P}_{3}=\frac{S^{3}}{\eta\left(P_{24}\right)}$, com representações

$$
\eta: P_{24} \rightarrow \operatorname{Aut}(\mathbb{Z})
$$

As possíveis representações para $P_{24}$ devem preservar as relações do grupo, ou seja, devem satisfazer as equações:

- $\eta(x)^{2}=\eta\left(x^{2}\right)=\eta\left(y^{2}\right)=\eta(y)^{2}$

- $\eta(y x y)=\eta(y) \eta(x) \eta(y)=\eta(x)$

- $\eta(z x)=\eta(z) \eta(x)=\eta(y) \eta(z)=\eta(y z)$

- $\eta(z y)=\eta(z) \eta(y)=\eta(x) \eta(y) \eta(z)=\eta(x y z)$,

- $\eta(z)^{3}=\eta\left(z^{3}\right)=\eta(1)=1$,

- $\eta(x)^{4}=\eta\left(x^{4}\right)=\eta(1)=1$.

Podemos observar que apenas a representação trivial, isto é, a representação $\eta:(x, y, z) \rightarrow(1,1,1)$, satisfaz as condições anteriores. Dessa forma, esta será a única representação considerada no cálculo dos grupos de homologia.

Consideremos o complexo de cadeias abaixo:

$0 \longrightarrow \mathbb{Z} P_{24}\left[\widetilde{c}_{3,1}\right] \stackrel{\partial_{3}}{\longrightarrow} \mathbb{Z} P_{24}\left[\widetilde{c}_{2,1}, \widetilde{c}_{2,2}, \widetilde{c}_{2,3}, \widetilde{c}_{2,4}\right] \stackrel{\partial_{2}}{\longrightarrow} \mathbb{Z} P_{24}\left[\widetilde{c}_{1,1}, \widetilde{c}_{1,2}, \widetilde{c}_{1,3}, \widetilde{c}_{1,4}\right] \stackrel{\partial_{1}}{\longrightarrow} \mathbb{Z} P_{24}\left[\widetilde{c}_{0,1}\right] \longrightarrow 0$ 
Com relação a representação trivial, os operadores bordos mencionados no complexo de cadeias acima são:

$$
\begin{aligned}
& \partial_{1}\left(\widetilde{c}_{1,1}\right)=0 \\
& \partial_{1}\left(\widetilde{c}_{1,2}\right)=0 \\
& \partial_{1}\left(\widetilde{c}_{1,3}\right)=0 \\
& \partial_{1}\left(\widetilde{c}_{1,4}\right)=0 \\
& \partial_{2}\left(\widetilde{c}_{2,1}\right)=-\widetilde{c}_{1,1}+\widetilde{c}_{1,2}-\widetilde{c}_{1,3} \\
& \partial_{2}\left(\widetilde{c}_{2,2}\right)=-\widetilde{c}_{1,1}-\widetilde{c}_{1,3}+\widetilde{c}_{1,4} \\
& \partial_{2}\left(\widetilde{c}_{2,3}\right)=-\widetilde{c}_{1,2}+\widetilde{c}_{1,3}-\widetilde{c}_{1,4} \\
& \partial_{2}\left(\widetilde{c}_{2,4}\right)=\widetilde{c}_{1,1}-\widetilde{c}_{1,2}-\widetilde{c}_{1,4} \\
& \partial_{3}\left(\widetilde{c}_{3,1}\right)=0 .
\end{aligned}
$$

Nas subseções a seguir, utilizamos o complexo de cadeias, apresentado anteriormente, para calcular os grupos de homologia da forma espacial esférica tetraedral, de dimensão três, com coeficientes em $\mathbb{Z}, \mathbb{Z}_{2}, \mathbb{Z}_{3}$ e $\mathbb{Z}_{p}$, com $p$ primo e $p>3$.

\subsubsection{Grupos de homologia com coeficientes em $\mathbb{Z}$}

- $H_{0}\left(\mathscr{P}_{3} ; \mathbb{Z}\right)$

Como $\left[\partial_{1}\right]=\left(\begin{array}{l}0 \\ 0 \\ 0\end{array}\right)$ temos $I m \partial_{1}=\{0\}$ e sendo $\partial_{0}=0$ então $K e r \partial_{0} \simeq \mathbb{Z}$. Logo,

$$
H_{0}\left(\mathscr{P}_{3} ; \mathbb{Z}\right)=\frac{\operatorname{Ker} \partial_{0}}{\operatorname{Im} \partial_{1}}=\frac{\mathbb{Z}}{\{0\}}=\mathbb{Z}
$$

- $H_{1}\left(\mathscr{P}_{3} ; \mathbb{Z}\right)$

$$
\begin{aligned}
H_{1}\left(\mathscr{P}_{3} ; \mathbb{Z}\right) & =\frac{\operatorname{Ker} \partial_{1}}{\operatorname{Im} \partial_{2}} \\
& =\frac{\mathbb{Z}\left[\widetilde{c}_{1,1}, \widetilde{c}_{1,2}, \widetilde{c}_{1,3}, \widetilde{c}_{1,4}\right]}{\mathbb{Z}\left[-\widetilde{c}_{1,1}+\widetilde{c}_{1,2}-\widetilde{c}_{1,3},-\widetilde{c}_{1,1}-\widetilde{c}_{1,3}+\widetilde{c}_{1,4},-\widetilde{c}_{1,2}+\widetilde{c}_{1,3}-\widetilde{c}_{1,4}, \widetilde{c}_{1,1}-\widetilde{c}_{1,2}-\widetilde{c}_{1,4}\right]}
\end{aligned}
$$


Consideremos

$$
\left[\partial_{2}\right]=\left(\begin{array}{cccc}
-1 & 1 & -1 & 0 \\
-1 & 0 & -1 & 1 \\
0 & -1 & 1 & -1 \\
1 & -1 & 0 & -1
\end{array}\right)
$$

Fazendo a mudança de base de $\left\{\widetilde{c}_{1,1}, \widetilde{c}_{1,2}, \widetilde{c}_{1,3}, \widetilde{c}_{1,4}\right\}$ para $\left\{\widetilde{c}_{1,4}, \widetilde{c}_{1,1}+\widetilde{c}_{1,4}, \widetilde{c}_{1,2}+\widetilde{c}_{1,4}, \widetilde{c}_{1,3}\right\}=$ $=\left\{\omega_{1}, \omega_{2}, \omega_{3}, \omega_{4}\right\}$, temos

$$
\left(\begin{array}{llll}
0 & 0 & 0 & 1 \\
1 & 0 & 0 & 1 \\
0 & 1 & 0 & 1 \\
0 & 0 & 1 & 0
\end{array}\right)
$$

Notemos que a matriz mudança de base tem determinante 1.

$$
\begin{aligned}
& \partial\left(\widetilde{c}_{2,1}\right)=-\widetilde{c}_{1,1}+\widetilde{c}_{1,2}-\widetilde{c}_{1,3}=-\omega_{2}+\omega_{3}-\omega_{4} \\
& \partial\left(\widetilde{c}_{2,2}\right)=-\widetilde{c}_{1,1}-\widetilde{c}_{1,3}+\widetilde{c}_{1,4}=2 \omega_{1}-\omega_{3}-\omega_{4} \\
& \partial\left(\widetilde{c}_{2,3}\right)=-\widetilde{c}_{1,2}+\widetilde{c}_{1,3}-\widetilde{c}_{1,4}=-\omega_{3}+\omega_{4} \\
& \partial\left(\widetilde{c}_{2,3}\right)=\widetilde{c}_{1,1}-\widetilde{c}_{1,2}-\widetilde{c}_{1,4}=-\omega_{1}+\omega_{2}-\omega_{3}
\end{aligned}
$$

De fato,

$$
\left[\partial_{2}\right]=\left(\begin{array}{cccc}
-1 & 1 & -1 & 0 \\
-1 & 0 & -1 & 1 \\
0 & -1 & 1 & -1 \\
1 & -1 & 0 & -1
\end{array}\right) \sim\left(\begin{array}{cccc}
0 & -1 & 1 & -1 \\
2 & -1 & 0 & -1 \\
0 & 0 & -1 & 1 \\
-1 & 1 & -1 & 0
\end{array}\right)
$$

Logo,

$$
H_{1}\left(\mathscr{P}_{3} ; \mathbb{Z}\right)=\frac{\mathbb{Z}\left[\omega, \omega_{2}, \omega_{3}, \omega_{4}\right]}{\mathbb{Z}\left[-\omega_{2}+\omega_{3}-\omega_{4}, 2 \omega_{1}-\omega_{3}-\omega_{4},-\omega_{3}+\omega_{4},-\omega_{1}+\omega_{2}-\omega_{3}\right]}
$$

Agora, fazendo a mudança de base de $\left\{\widetilde{c}_{2,1}, \widetilde{c}_{2,2}, \widetilde{c}_{2,3}, \widetilde{c}_{2,4}\right\}$ para $\left\{-\widetilde{c}_{2,1}+\widetilde{c}_{2,2},-\widetilde{c}_{2,1}-\widetilde{c}_{2,3}, \widetilde{c}_{2,3},-\widetilde{c}_{2,1}-\widetilde{c}_{2,4}\right\}=\left\{\psi_{1}, \psi_{2}, \psi_{3}, \psi_{4}\right\}$, obtemos 


$$
\left(\begin{array}{cccc}
-1 & 1 & 0 & 0 \\
-1 & 0 & -1 & 0 \\
0 & 0 & 1 & 0 \\
-1 & 0 & 0 & -1
\end{array}\right) \simeq\left(\begin{array}{cccc}
-1 & 0 & -1 & 0 \\
-1 & 1 & 0 & 0 \\
0 & 0 & 1 & 0 \\
-1 & 0 & 0 & -1
\end{array}\right)
$$

Observemos que a matriz possui determinante 1.

Assim,

$$
\begin{aligned}
& \partial\left(\psi_{1}\right)=\partial\left(-\widetilde{c}_{2,1}+\widetilde{c}_{2,2}\right)=2 \omega_{1}-\omega_{3} \\
& \partial\left(\psi_{2}\right)=\partial\left(-\widetilde{c}_{2,1}-\widetilde{c}_{2,3}\right)=\omega_{2} \\
& \partial\left(\psi_{3}\right)=\partial\left(\widetilde{c}_{2,3}\right)=-\omega_{3}+\omega_{4} \\
& \partial\left(\psi_{4}\right)=\partial\left(-\widetilde{c}_{2,1}-\widetilde{c}_{2,4}\right)=\omega_{1}+\omega_{4} .
\end{aligned}
$$

Logo,

$$
H_{1}\left(\mathscr{P}_{3} ; \mathbb{Z}\right)=\frac{\mathbb{Z}\left[\omega_{1}, \omega_{2}, \omega_{3}, \omega_{4}\right]}{\mathbb{Z}\left[2 \omega_{1}-\omega_{3}, \omega_{2},-\omega_{3}+\omega_{4}, \omega_{1}+\omega_{4}\right]} \simeq \frac{\mathbb{Z}\left[\omega_{1}, \omega_{3}, \omega_{4}\right]}{\mathbb{Z}\left[2 \omega_{1}-\omega_{3},-\omega_{3}+\omega_{4}, \omega_{1}+\omega_{4}\right]}
$$

Fazendo a mudança de base de $\left\{\psi_{1}, \psi_{3}, \psi_{4}\right\}$ para $\left\{\psi_{1}-\psi_{3}+\psi_{4}, \psi_{3}, \psi_{4}\right\}=\left\{\sigma_{1}, \sigma_{2}, \sigma_{3}\right\}$, obtemos a matriz

$$
\left(\begin{array}{ccc}
1 & -1 & 1 \\
0 & 1 & 0 \\
0 & 0 & 1
\end{array}\right)
$$

que tem determinante 1 . Desse modo,

$$
\begin{aligned}
& \partial\left(\sigma_{1}\right)=\partial\left(\psi_{1}-\psi_{3}+\psi_{4}\right)=3 \omega_{1} \\
& \partial\left(\sigma_{2}\right)=\partial\left(\psi_{3}\right)=-\omega_{3}+\omega_{4} \\
& \partial\left(\sigma_{3}\right)=\partial\left(\psi_{4}\right)=\omega_{1}+\omega_{4} .
\end{aligned}
$$

Assim,

$$
H_{1}\left(\mathscr{P}_{3} ; \mathbb{Z}\right)=\frac{\mathbb{Z}\left[\omega_{1}, \omega_{2}, \omega_{3}\right]}{\mathbb{Z}\left[3 \omega_{1},-\omega_{3}+\omega_{4}, \omega_{1}+\omega_{4}\right]} .
$$

Por fim, fazendo a mudança de base de $\left\{\omega_{1}, \omega_{3}, \omega_{4}\right\}$ para $\left\{\omega_{1},-\omega_{3}+\omega_{4}, \omega_{1}+\omega_{4}\right\}=$ $=\left\{\delta_{1}, \delta_{2}, \delta_{3}\right\}$, temos 


$$
\left(\begin{array}{ccc}
1 & 0 & 0 \\
0 & -1 & 1 \\
1 & 0 & 1
\end{array}\right)
$$

A matriz mudança de base possui determinante -1 .

Assim,

$\partial\left(\sigma_{1}\right)=3 \omega_{1}=3 \delta_{1}$

$\partial\left(\sigma_{2}\right)=-\omega_{3}+\omega_{4}=\delta_{2}$

$\partial\left(\sigma_{3}\right)=\omega_{1}+\omega_{4}=\delta_{3}$.

Então,

$$
H_{1}\left(\mathscr{P}_{3} ; \mathbb{Z}\right)=\frac{\mathbb{Z}\left[\delta_{1}, \delta_{2}, \delta_{3}\right]}{\mathbb{Z}\left[3 \delta_{1}, \delta_{2}, \delta_{3}\right]} \simeq \frac{\mathbb{Z}\left[\delta_{1}\right]}{\mathbb{Z}\left[3 \delta_{1}\right]} \simeq \mathbb{Z}_{3}
$$

- $H_{2}\left(\mathscr{P}_{3} ; \mathbb{Z}\right)$

Como $\left[\partial_{3}\right]=\left(\begin{array}{cccc}0 & 0 & 0 & 0\end{array}\right)$ temos $\operatorname{Im} \partial_{3}=\{0\}$. Além disso,

$$
\left[\partial_{2}\right]=\left(\begin{array}{cccc}
-1 & 1 & -1 & 0 \\
-1 & 0 & -1 & 1 \\
0 & -1 & 1 & -1 \\
1 & -1 & 0 & -1
\end{array}\right)
$$

possui o determinante não nulo, portanto $\partial_{2}$ é injetor, ou seja, $\operatorname{Ker} \partial_{2}=\{0\}$. Assim,

$$
H_{2}\left(\mathscr{P}_{3} ; \mathbb{Z}\right)=\frac{K e r \partial_{2}}{\operatorname{Im} \partial_{3}}=\frac{\{0\}}{\{0\}}=0 .
$$

- $H_{3}\left(\mathscr{P}_{3} ; \mathbb{Z}\right)$

$$
H_{3}\left(\mathscr{P}_{3} ; \mathbb{Z}\right)=\frac{\operatorname{Ker} \partial_{3}}{\operatorname{Im} \partial_{4}}=\frac{\mathbb{Z}}{\{0\}}=\mathbb{Z}, \text { pois }\left[\partial_{3}\right]=\left(\begin{array}{ccc}
0 & 0 & 0
\end{array}\right) .
$$


Portanto,

$$
H_{i}\left(\mathscr{P}_{3} ; \mathbb{Z}\right)= \begin{cases}\mathbb{Z}, & \mathrm{i}=0,3 \\ \mathbb{Z}_{3}, & \mathrm{i}=1 ; \\ 0, & \mathrm{i}=2 .\end{cases}
$$

\subsubsection{Grupos de homologia com coeficientes em $\mathbb{Z}_{2}$}

- $H_{0}\left(\mathscr{P}_{3} ; \mathbb{Z}_{2}\right)$

$$
\begin{gathered}
\text { Como }\left[\partial_{1}\right]=\left(\begin{array}{l}
0 \\
0 \\
0
\end{array}\right) \text { temos } \operatorname{Im} \partial_{1}=\{0\} \text { e sendo } \partial_{0}=0 \text { então } K e r \partial_{0} \simeq \mathbb{Z}_{2} \text {. Logo, } \\
H_{0}\left(\mathscr{P}_{3} ; \mathbb{Z}_{2}\right)=\frac{\operatorname{Ker} \partial_{0}}{\operatorname{Im} \partial_{1}}=\frac{\mathbb{Z}_{2}}{\{0\}}=\mathbb{Z}_{2} .
\end{gathered}
$$

- $H_{1}\left(\mathscr{P}_{3} ; \mathbb{Z}_{2}\right)$

$$
\begin{aligned}
H_{1}\left(\mathscr{P}_{3} ; \mathbb{Z}_{2}\right) & =\frac{\operatorname{Ker} \partial_{1}}{\operatorname{Im} \partial_{2}} \\
= & \frac{\mathbb{Z}_{2}\left[\widetilde{c}_{1,1}, \widetilde{c}_{1,2}, \widetilde{c}_{1,3}, \widetilde{c}_{1,4}\right]}{\mathbb{Z}_{2}\left[-\widetilde{c}_{1,1}+\widetilde{c}_{1,2}-\widetilde{c}_{1,3},-\widetilde{c}_{1,1}-\widetilde{c}_{1,3}+\widetilde{c}_{1,4},-\widetilde{c}_{1,2}+\widetilde{c}_{1,3}-\widetilde{c}_{1,4}, \widetilde{c}_{1,1}-\widetilde{c}_{1,2}-\widetilde{c}_{1,4}\right]}
\end{aligned}
$$

Consideremos

$$
\left[\partial_{2}\right]=\left(\begin{array}{cccc}
-1 & 1 & -1 & 0 \\
-1 & 0 & -1 & 1 \\
0 & -1 & 1 & -1 \\
1 & -1 & 0 & -1
\end{array}\right) .
$$

Fazendo a mudança de base de $\left\{\widetilde{c}_{1,1}, \widetilde{c}_{1,2}, \widetilde{c}_{1,3}, \widetilde{c}_{1,4}\right\}$ para $\left\{\widetilde{c}_{1,4}, \widetilde{c}_{1,1}+\widetilde{c}_{1,4}, \widetilde{c}_{1,2}+\widetilde{c}_{1,4}, \widetilde{c}_{1,3}\right\}=$ $=\left\{\omega_{1}, \omega_{2}, \omega_{3}, \omega_{4}\right\}$, temos

$$
\left(\begin{array}{llll}
0 & 0 & 0 & 1 \\
1 & 0 & 0 & 1 \\
0 & 1 & 0 & 1 \\
0 & 0 & 1 & 0
\end{array}\right)
$$


Notemos que a matriz mudança de base tem determinante 1 .

$\partial\left(\widetilde{c}_{2,1}\right)=-\widetilde{c}_{1,1}+\widetilde{c}_{1,2}-\widetilde{c}_{1,3}=-\omega_{2}+\omega_{3}-\omega_{4}$

$\partial\left(\widetilde{c}_{2,2}\right)=-\widetilde{c}_{1,1}-\widetilde{c}_{1,3}+\widetilde{c}_{1,4}=2 \omega_{1}-\omega_{3}-\omega_{4}=-\omega_{3}-\omega_{4}$

$\partial\left(\widetilde{c}_{2,3}\right)=-\widetilde{c}_{1,2}+\widetilde{c}_{1,3}-\widetilde{c}_{1,4}=-\omega_{3}+\omega_{4}$

$\partial\left(\widetilde{c}_{2,3}\right)=\widetilde{c}_{1,1}-\widetilde{c}_{1,2}-\widetilde{c}_{1,4}=-\omega_{1}+\omega_{2}-\omega_{3}$

De fato,

$\left[\partial_{2}\right]=\left(\begin{array}{cccc}-1 & 1 & -1 & 0 \\ -1 & 0 & -1 & 1 \\ 0 & -1 & 1 & -1 \\ 1 & -1 & 0 & -1\end{array}\right) \sim\left(\begin{array}{cccc}0 & -1 & 1 & -1 \\ 2 & -1 & 0 & -1 \\ 0 & 0 & -1 & 1 \\ -1 & 1 & -1 & 0\end{array}\right) \sim\left(\begin{array}{cccc}0 & -1 & 1 & -1 \\ 0 & -1 & 0 & -1 \\ 0 & 0 & -1 & 1 \\ -1 & 1 & -1 & 0\end{array}\right)$

Logo,

$$
H_{1}\left(\mathscr{P}_{3} ; \mathbb{Z}_{2}\right)=\frac{\mathbb{Z}_{2}\left[\omega_{1}, \omega_{2}, \omega_{3}, \omega_{4}\right]}{\mathbb{Z}\left[-\omega_{2}+\omega_{3}-\omega_{4},-\omega_{3}-\omega_{4},-\omega_{3}+\omega_{4},-\omega_{1}+\omega_{2}-\omega_{3}\right]}
$$

Agora, fazendo a mudança de base de $\left\{\widetilde{c}_{2,1}, \widetilde{c}_{2,2}, \widetilde{c}_{2,3}, \widetilde{c}_{2,4}\right\}$ para $\left\{-\widetilde{c}_{2,1}+\widetilde{c}_{2,2},-\widetilde{c}_{2,1}-\widetilde{c}_{2,3}, \widetilde{c}_{2,3},-\widetilde{c}_{2,1}-\widetilde{c}_{2,4}\right\}=\left\{\psi_{1}, \psi_{2}, \psi_{3}, \psi_{4}\right\}$, obtemos

$$
\left(\begin{array}{cccc}
-1 & 1 & 0 & 0 \\
-1 & 0 & -1 & 0 \\
0 & 0 & 1 & 0 \\
-1 & 0 & 0 & -1
\end{array}\right) \simeq\left(\begin{array}{cccc}
-1 & 0 & -1 & 0 \\
-1 & 1 & 0 & 0 \\
0 & 0 & 1 & 0 \\
-1 & 0 & 0 & -1
\end{array}\right)
$$

Observemos que a matriz tem determinante 1 .

Assim,

$$
\begin{aligned}
& \partial\left(\psi_{1}\right)=\partial\left(-\widetilde{c}_{2,1}+\widetilde{c}_{2,2}\right)=-\omega_{3} \\
& \partial\left(\psi_{2}\right)=\partial\left(-\widetilde{c}_{2,1}-\widetilde{c}_{2,3}\right)=\omega_{2} \\
& \partial\left(\psi_{3}\right)=\partial\left(\widetilde{c}_{2,3}\right)=-\omega_{3}+\omega_{4} \\
& \partial\left(\psi_{4}\right)=\partial\left(-\widetilde{c}_{2,1}-\widetilde{c}_{2,4}\right)=\omega_{1}+\omega_{4} .
\end{aligned}
$$


Logo,

$$
H_{1}\left(\mathscr{P}_{3} ; \mathbb{Z}_{2}\right)=\frac{\mathbb{Z}_{2}\left[\omega_{1}, \omega_{2}, \omega_{3}, \omega_{4}\right]}{\mathbb{Z}_{2}\left[-\omega_{3}, \omega_{2},-\omega_{3}+\omega_{4}, \omega_{1}+\omega_{4}\right]} \simeq \frac{\mathbb{Z}_{2}\left[\omega_{1}, \omega_{3}, \omega_{4}\right]}{\mathbb{Z}_{2}\left[-\omega_{3},-\omega_{3}+\omega_{4}, \omega_{1}+\omega_{4}\right]}
$$

Mudando a base de $\left\{\psi_{1}, \psi_{3}, \psi_{4}\right\}$ para $\left\{\psi_{1}-\psi_{3}+\psi_{4}, \psi_{3}, \psi_{4}\right\}=\left\{\sigma_{1}, \sigma_{2}, \sigma_{3}\right\}$, obtemos a matriz

$$
\left(\begin{array}{ccc}
1 & -1 & 1 \\
0 & 1 & 0 \\
0 & 0 & 1
\end{array}\right)
$$

que possui determinante 1. Dessa forma,

$$
\begin{aligned}
& \partial\left(\sigma_{1}\right)=\partial\left(\psi_{1}-\psi_{3}+\psi_{4}\right)=\omega_{1} \\
& \partial\left(\sigma_{2}\right)=\partial\left(\psi_{3}\right)=-\omega_{3}+\omega_{4} \\
& \partial\left(\sigma_{3}\right)=\partial\left(\psi_{4}\right)=\omega_{1}+\omega_{4} .
\end{aligned}
$$

Assim,

$$
H_{1}\left(\mathscr{P}_{3} ; \mathbb{Z}_{2}\right)=\frac{\mathbb{Z}_{2}\left[\omega_{1}, \omega_{2}, \omega_{3}\right]}{\mathbb{Z}_{2}\left[\omega_{1},-\omega_{3}+\omega_{4}, \omega_{1}+\omega_{4}\right]} .
$$

Por fim, fazendo a mudança de base de $\left\{\omega_{1}, \omega_{3}, \omega_{4}\right\}$ para $\left\{\omega_{1},-\omega_{3}+\omega_{4}, \omega_{1}+\omega_{4}\right\}=$ $=\left\{\delta_{1}, \delta_{2}, \delta_{3}\right\}$, temos

$$
\left(\begin{array}{ccc}
1 & 0 & 0 \\
0 & -1 & 1 \\
1 & 0 & 1
\end{array}\right)
$$

A matriz mudança de base possui determinante -1 .

Desse modo,

$$
\begin{aligned}
& \partial\left(\sigma_{1}\right)=\omega_{1}=\delta_{1} \\
& \partial\left(\sigma_{2}\right)=-\omega_{3}+\omega_{4}=\delta_{2} \\
& \partial\left(\sigma_{3}\right)=\omega_{1}+\omega_{4}=\delta_{3} .
\end{aligned}
$$

Portanto,

$$
H_{1}\left(\mathscr{P}_{3} ; \mathbb{Z}_{2}\right)=\frac{\mathbb{Z}_{2}\left[\delta_{1}, \delta_{2}, \delta_{3}\right]}{\mathbb{Z}_{2}\left[\delta_{1}, \delta_{2}, \delta_{3}\right]} \simeq \frac{\mathbb{Z}_{2}\left[\delta_{1}\right]}{\mathbb{Z}_{2}\left[\delta_{1}\right]} \simeq 0
$$


- $H_{2}\left(\mathscr{P}_{3} ; \mathbb{Z}_{2}\right)$

Como $\left[\partial_{3}\right]=\left(\begin{array}{llll}0 & 0 & 0 & 0\end{array}\right)$ temos $\operatorname{Im}_{3}=\{0\}$. Além disso,

$$
\left[\partial_{2}\right]=\left(\begin{array}{cccc}
-1 & 1 & -1 & 0 \\
-1 & 0 & -1 & 1 \\
0 & -1 & 1 & -1 \\
1 & -1 & 0 & -1
\end{array}\right)
$$

possui o determinante $-3 \neq 0 \in \mathbb{Z}_{2}$, portanto $\partial_{2}$ é injetor, ou seja, $\operatorname{Ker} \partial_{2}=\{0\}$. Assim,

$$
H_{2}\left(\mathscr{P}_{3} ; \mathbb{Z}_{2}\right)=\frac{K e r \partial_{2}}{\operatorname{Im} \partial_{3}}=\frac{\{0\}}{\{0\}}=0
$$

- $H_{3}\left(\mathscr{P}_{3} ; \mathbb{Z}_{2}\right)$

$$
H_{3}\left(\mathscr{P}_{3} ; \mathbb{Z}_{2}\right)=\frac{\operatorname{Ker} \partial_{3}}{\operatorname{Im} \partial_{4}}=\frac{\mathbb{Z}_{2}}{\{0\}}=\mathbb{Z}_{2}, \text { pois }\left[\partial_{3}\right]=\left(\begin{array}{ccc}
0 & 0 & 0
\end{array}\right) .
$$

Portanto,

$$
H_{i}\left(\mathscr{P}_{3} ; \mathbb{Z}_{2}\right)= \begin{cases}\mathbb{Z}_{2}, & \mathrm{i}=0,3 \\ 0, & \mathrm{i}=1,2\end{cases}
$$

\subsubsection{Grupos de homologia com coeficientes em $\mathbb{Z}_{3}$}

- $H_{0}\left(\mathscr{P}_{3} ; \mathbb{Z}_{3}\right)$

$$
\text { Como }\left[\partial_{1}\right]=\left(\begin{array}{l}
0 \\
0 \\
0
\end{array}\right) \text { temos } I m \partial_{1}=\{0\} \text { e sendo } \partial_{0}=0 \text { então } K e r \partial_{0} \simeq \mathbb{Z}_{3} \text {. Desse }
$$

modo,

$$
H_{0}\left(\mathscr{P}_{3} ; \mathbb{Z}_{3}\right)=\frac{\operatorname{Ker} \partial_{0}}{\operatorname{Im} \partial_{1}}=\frac{\mathbb{Z}_{3}}{\{0\}}=\mathbb{Z}_{3} .
$$

- $H_{1}\left(\mathscr{P}_{3} ; \mathbb{Z}_{3}\right)$ 


$$
\begin{aligned}
H_{1}\left(\mathscr{P}_{3} ; \mathbb{Z}_{3}\right) & =\frac{\operatorname{Ker} \partial_{1}}{\operatorname{Im} \partial_{2}} \\
= & \frac{\mathbb{Z}_{3}\left[\widetilde{c}_{1,1}, \widetilde{c}_{1,2}, \widetilde{c}_{1,3}, \widetilde{c}_{1,4}\right]}{\mathbb{Z}_{3}\left[-\widetilde{c}_{1,1}+\widetilde{c}_{1,2}-\widetilde{c}_{1,3},-\widetilde{c}_{1,1}-\widetilde{c}_{1,3}+\widetilde{c}_{1,4},-\widetilde{c}_{1,2}+\widetilde{c}_{1,3}-\widetilde{c}_{1,4}, \widetilde{c}_{1,1}-\widetilde{c}_{1,2}-\widetilde{c}_{1,4}\right]}
\end{aligned}
$$

\section{Consideremos}

$$
\left[\partial_{2}\right]=\left(\begin{array}{cccc}
-1 & 1 & -1 & 0 \\
-1 & 0 & -1 & 1 \\
0 & -1 & 1 & -1 \\
1 & -1 & 0 & -1
\end{array}\right)
$$

Fazendo a mudança de base de $\left\{\widetilde{c}_{1,1}, \widetilde{c}_{1,2}, \widetilde{c}_{1,3}, \widetilde{c}_{1,4}\right\}$ para $\left\{\widetilde{c}_{1,4}, \widetilde{c}_{1,1}+\widetilde{c}_{1,4}, \widetilde{c}_{1,2}+\widetilde{c}_{1,4}, \widetilde{c}_{1,3}\right\}=$ $=\left\{\omega_{1}, \omega_{2}, \omega_{3}, \omega_{4}\right\}$, temos

$$
\left(\begin{array}{llll}
0 & 0 & 0 & 1 \\
1 & 0 & 0 & 1 \\
0 & 1 & 0 & 1 \\
0 & 0 & 1 & 0
\end{array}\right)
$$

Notemos que a matriz mudança de base tem determinante 1 .

$$
\begin{aligned}
& \partial\left(\widetilde{c}_{2,1}\right)=-\widetilde{c}_{1,1}+\widetilde{c}_{1,2}-\widetilde{c}_{1,3}=-\omega_{2}+\omega_{3}-\omega_{4} \\
& \partial\left(\widetilde{c}_{2,2}\right)=-\widetilde{c}_{1,1}-\widetilde{c}_{1,3}+\widetilde{c}_{1,4}=2 \omega_{1}-\omega_{3}-\omega_{4} \\
& \partial\left(\widetilde{c}_{2,3}\right)=-\widetilde{c}_{1,2}+\widetilde{c}_{1,3}-\widetilde{c}_{1,4}=-\omega_{3}+\omega_{4} \\
& \partial\left(\widetilde{c}_{2,3}\right)=\widetilde{c}_{1,1}-\widetilde{c}_{1,2}-\widetilde{c}_{1,4}=-\omega_{1}+\omega_{2}-\omega_{3}
\end{aligned}
$$

De fato,

$$
\left[\partial_{2}\right]=\left(\begin{array}{cccc}
-1 & 1 & -1 & 0 \\
-1 & 0 & -1 & 1 \\
0 & -1 & 1 & -1 \\
1 & -1 & 0 & -1
\end{array}\right) \sim\left(\begin{array}{cccc}
0 & -1 & 1 & -1 \\
2 & -1 & 0 & -1 \\
0 & 0 & -1 & 1 \\
-1 & 1 & -1 & 0
\end{array}\right)
$$

Logo,

$$
H_{1}\left(\mathscr{P}_{3} ; \mathbb{Z}_{3}\right)=\frac{\mathbb{Z}_{3}\left[\omega, \omega_{2}, \omega_{3}, \omega_{4}\right]}{\mathbb{Z}_{3}\left[-\omega_{2}+\omega_{3}-\omega_{4}, 2 \omega_{1}-\omega_{3}-\omega_{4},-\omega_{3}+\omega_{4},-\omega_{1}+\omega_{2}-\omega_{3}\right]} .
$$

Agora, fazendo a mudança de base de $\left\{\widetilde{c}_{2,1}, \widetilde{c}_{2,2}, \widetilde{c}_{2,3}, \widetilde{c}_{2,4}\right\}$ para 
$\left\{-\widetilde{c}_{2,1}+\widetilde{c}_{2,2},-\widetilde{c}_{2,1}-\widetilde{c}_{2,3}, \widetilde{c}_{2,3},-\widetilde{c}_{2,1}-\widetilde{c}_{2,4}\right\}=\left\{\psi_{1}, \psi_{2}, \psi_{3}, \psi_{4}\right\}$, obtemos

$$
\left(\begin{array}{cccc}
-1 & 1 & 0 & 0 \\
-1 & 0 & -1 & 0 \\
0 & 0 & 1 & 0 \\
-1 & 0 & 0 & -1
\end{array}\right) \simeq\left(\begin{array}{cccc}
-1 & 0 & -1 & 0 \\
-1 & 1 & 0 & 0 \\
0 & 0 & 1 & 0 \\
-1 & 0 & 0 & -1
\end{array}\right)
$$

Notemos que a matriz tem determinante 1.

Dessa forma,

$$
\begin{aligned}
& \partial\left(\psi_{1}\right)=\partial\left(-\widetilde{c}_{2,1}+\widetilde{c}_{2,2}\right)=2 \omega_{1}-\omega_{3} \\
& \partial\left(\psi_{2}\right)=\partial\left(-\widetilde{c}_{2,1}-\widetilde{c}_{2,3}\right)=\omega_{2} \\
& \partial\left(\psi_{3}\right)=\partial\left(\widetilde{c}_{2,3}\right)=-\omega_{3}+\omega_{4} \\
& \partial\left(\psi_{4}\right)=\partial\left(-\widetilde{c}_{2,1}-\widetilde{c}_{2,4}\right)=\omega_{1}+\omega_{4} .
\end{aligned}
$$

Logo,

$$
H_{1}\left(\mathscr{P}_{3} ; \mathbb{Z}_{3}\right)=\frac{\mathbb{Z}_{3}\left[\omega_{1}, \omega_{2}, \omega_{3}, \omega_{4}\right]}{\mathbb{Z}_{3}\left[2 \omega_{1}-\omega_{3}, \omega_{2},-\omega_{3}+\omega_{4}, \omega_{1}+\omega_{4}\right]} \simeq \frac{\mathbb{Z}_{3}\left[\omega_{1}, \omega_{3}, \omega_{4}\right]}{\mathbb{Z}_{3}\left[2 \omega_{1}-\omega_{3},-\omega_{3}+\omega_{4}, \omega_{1}+\omega_{4}\right]}
$$

Mudando a base de $\left\{\psi_{1}, \psi_{3}, \psi_{4}\right\}$ para $\left\{\psi_{1}-\psi_{3}+\psi_{4}, \psi_{3}, \psi_{4}\right\}=\left\{\sigma_{1}, \sigma_{2}, \sigma_{3}\right\}$, obtemos a matriz

$$
\left(\begin{array}{ccc}
1 & -1 & 1 \\
0 & 1 & 0 \\
0 & 0 & 1
\end{array}\right)
$$

que tem determinante 1. Desse modo,

$$
\begin{aligned}
& \partial\left(\sigma_{1}\right)=\partial\left(\psi_{1}-\psi_{3}+\psi_{4}\right)=3 \omega_{1}=0 \\
& \partial\left(\sigma_{2}\right)=\partial\left(\psi_{3}\right)=-\omega_{3}+\omega_{4} \\
& \partial\left(\sigma_{3}\right)=\partial\left(\psi_{4}\right)=\omega_{1}+\omega_{4} .
\end{aligned}
$$

Assim,

$$
H_{1}\left(\mathscr{P}_{3} ; \mathbb{Z}_{3}\right)=\frac{\mathbb{Z}_{3}\left[\omega_{1}, \omega_{2}, \omega_{3}\right]}{\mathbb{Z}_{3}\left[0,-\omega_{3}+\omega_{4}, \omega_{1}+\omega_{4}\right]}==\frac{\mathbb{Z}_{3}\left[\omega_{1}, \omega_{2}, \omega_{3}\right]}{\mathbb{Z}_{3}\left[-\omega_{3}+\omega_{4}, \omega_{1}+\omega_{4}\right]}
$$

Por fim, fazendo a mudança de base de $\left\{\omega_{1}, \omega_{3}, \omega_{4}\right\}$ para $\left\{\omega_{1},-\omega_{3}+\omega_{4}, \omega_{1}+\omega_{4}\right\}=$ 
$=\left\{\delta_{1}, \delta_{2}, \delta_{3}\right\}$, temos

$$
\left(\begin{array}{ccc}
1 & 0 & 0 \\
0 & -1 & 1 \\
1 & 0 & 1
\end{array}\right)
$$

A matriz mudança de base possui determinante -1 .

Logo,

$$
\begin{aligned}
& \partial\left(\sigma_{1}\right)=3 \omega_{1}=3 \delta_{1}=0 \\
& \partial\left(\sigma_{2}\right)=-\omega_{3}+\omega_{4}=\delta_{2} \\
& \partial\left(\sigma_{3}\right)=\omega_{1}+\omega_{4}=\delta_{3} .
\end{aligned}
$$

Então,

$$
H_{1}\left(\mathscr{P}_{3} ; \mathbb{Z}_{3}\right)=\frac{\mathbb{Z}_{3}\left[\delta_{1}, \delta_{2}, \delta_{3}\right]}{\mathbb{Z}_{3}\left[0, \delta_{2}, \delta_{3}\right]} \simeq \frac{\mathbb{Z}_{3}\left[\delta_{1}, \delta_{2}, \delta_{3}\right]}{\mathbb{Z}_{3}\left[\delta_{2}, \delta_{3}\right]} \simeq \mathbb{Z}_{3}\left[\delta_{1}\right] \simeq \mathbb{Z}_{3}
$$

- $H_{2}\left(\mathscr{P}_{3} ; \mathbb{Z}_{3}\right)$

Notemos que $\left[\partial_{3}\right]=\left(\begin{array}{cccc}0 & 0 & 0 & 0\end{array}\right)$ assim, $\operatorname{Im} \partial_{3}=\{0\}$. Agora,

$$
\left[\partial_{2}\right]=\left(\begin{array}{cccc}
-1 & 1 & -1 & 0 \\
-1 & 0 & -1 & 1 \\
0 & -1 & 1 & -1 \\
1 & -1 & 0 & -1
\end{array}\right)
$$

possui o determinante $-3=0 \in \mathbb{Z}_{3}$.

Analisemos $\mathrm{Ker} \partial_{2}$.

Seja $\sigma$ uma 2-cadeia representada por $\left(a_{1}, a_{2}, a_{3}, a_{4}\right)$, temos

$$
\begin{aligned}
\partial_{2}(\sigma) & =a_{1}\left(-\widetilde{c}_{1,1}+\widetilde{c}_{1,2}-\widetilde{c}_{1,3}\right)+a_{2}\left(-\widetilde{c}_{1,1}-\widetilde{c}_{1,3}+\widetilde{c}_{1,4}\right)+a_{3}\left(-\widetilde{c}_{1,2}+\widetilde{c}_{1,3}-\widetilde{c}_{1,4}\right)+ \\
& +a_{4}\left(\widetilde{c}_{1,1}-\widetilde{c}_{1,2}-\widetilde{c}_{1,4}\right) \\
\partial_{2}(\sigma) & =\left(-a_{1}-a_{2}+a_{4}\right) \widetilde{c}_{1,1}+\left(a_{1}-a_{3}-a_{4}\right) \widetilde{c}_{1,2}+\left(-a_{1}-a_{2}+a_{3}\right) \widetilde{c}_{1,3}+\left(a_{2}-a_{3}-a_{4}\right) \widetilde{c}_{1,4}
\end{aligned}
$$




$$
\sigma \in \operatorname{Ker} \partial_{2} \Longleftrightarrow\left\{\begin{array}{l}
-a_{1}-a_{2}+a_{4}=0 \\
a_{1}-a_{3}-a_{4}=0 \\
-a_{1}-a_{2}+a_{3}=0 \\
a_{2}-a_{3}-a_{4}=0
\end{array}\right.
$$

Resolvendo o sistema, obtemos $a_{1}=a_{2}=-a_{3}=-a_{4}$.

Logo, $\sigma=a_{1} \widetilde{c}_{2,1}+a_{1} \widetilde{c}_{2,2}-a_{1} \widetilde{c}_{2,3}-a_{1} \widetilde{c}_{2,4}=a_{1}\left(\widetilde{c}_{2,1}+\widetilde{c}_{2,2}-\widetilde{c}_{2,3}-\widetilde{c}_{2,4}\right)$.

Portanto,

$$
H_{2}\left(\mathscr{P}_{3} ; \mathbb{Z}_{3}\right)=\frac{\operatorname{Ker} \partial_{2}}{\operatorname{Im} \partial_{3}}=\frac{\mathbb{Z}_{3}\left[\widetilde{c}_{2,1}+\widetilde{c}_{2,2}-\widetilde{c}_{2,3}-\widetilde{c}_{2,4}\right]}{\{0\}}=\mathbb{Z}_{3}
$$

- $H_{3}\left(\mathscr{P}_{3} ; \mathbb{Z}_{3}\right)$

$$
H_{3}\left(\mathscr{P}_{3} ; \mathbb{Z}_{3}\right)=\frac{\operatorname{Ker} \partial_{3}}{\operatorname{Im} \partial_{4}}=\frac{\mathbb{Z}_{3}}{\{0\}}=\mathbb{Z}_{3} \text {, pois }\left[\partial_{3}\right]=\left(\begin{array}{ccc}
0 & 0 & 0
\end{array}\right) .
$$

Portanto, $H_{i}\left(\mathscr{P}_{3} ; \mathbb{Z}_{3}\right)=\mathbb{Z}_{3}, i=0,1,2,3$.

\subsubsection{Grupos de homologia com coeficientes em $\mathbb{Z}_{p}$, com $p$ primo} e $p>3$

- $H_{0}\left(\mathscr{P}_{3} ; \mathbb{Z}_{p}\right)$

$$
\begin{gathered}
\text { Como }\left[\partial_{1}\right]=\left(\begin{array}{l}
0 \\
0 \\
0
\end{array}\right) \text { temos } \operatorname{Im} \partial_{1}=\{0\} \text { e sendo } \partial_{0}=0 \text { então } K e r \partial_{0} \simeq \mathbb{Z}_{p} \text {. Logo, } \\
H_{0}\left(\mathscr{P}_{3} ; \mathbb{Z}_{p}\right)=\frac{\operatorname{Ker} \partial_{0}}{\operatorname{Im} \partial_{1}}=\frac{\mathbb{Z}_{p}}{\{0\}}=\mathbb{Z}_{p} .
\end{gathered}
$$


- $H_{1}\left(\mathscr{P}_{3} ; \mathbb{Z}_{p}\right)$

$$
\begin{aligned}
H_{1}\left(\mathscr{P}_{3} ; \mathbb{Z}_{p}\right) & =\frac{\operatorname{Ker} \partial_{1}}{\operatorname{Im} \partial_{2}} \\
& =\frac{\mathbb{Z}_{p}\left[\widetilde{c}_{1,1}, \widetilde{c}_{1,2}, \widetilde{c}_{1,3}, \widetilde{c}_{1,4}\right]}{\mathbb{Z}_{p}\left[-\widetilde{c}_{1,1}+\widetilde{c}_{1,2}-\widetilde{c}_{1,3},-\widetilde{c}_{1,1}-\widetilde{c}_{1,3}+\widetilde{c}_{1,4},-\widetilde{c}_{1,2}+\widetilde{c}_{1,3}-\widetilde{c}_{1,4}, \widetilde{c}_{1,1}-\widetilde{c}_{1,2}-\widetilde{c}_{1,4}\right]}
\end{aligned}
$$

\section{Consideremos}

$$
\left[\partial_{2}\right]=\left(\begin{array}{cccc}
-1 & 1 & -1 & 0 \\
-1 & 0 & -1 & 1 \\
0 & -1 & 1 & -1 \\
1 & -1 & 0 & -1
\end{array}\right)
$$

Fazendo a mudança de base de $\left\{\widetilde{c}_{1,1}, \widetilde{c}_{1,2}, \widetilde{c}_{1,3}, \widetilde{c}_{1,4}\right\}$ para $\left\{\widetilde{c}_{1,4}, \widetilde{c}_{1,1}+\widetilde{c}_{1,4}, \widetilde{c}_{1,2}+\widetilde{c}_{1,4}, \widetilde{c}_{1,3}\right\}=$ $=\left\{\omega_{1}, \omega_{2}, \omega_{3}, \omega_{4}\right\}$, temos

$$
\left(\begin{array}{llll}
0 & 0 & 0 & 1 \\
1 & 0 & 0 & 1 \\
0 & 1 & 0 & 1 \\
0 & 0 & 1 & 0
\end{array}\right)
$$

Observemos que a matriz mudança de base tem determinante 1 .

$$
\begin{aligned}
& \partial\left(\widetilde{c}_{2,1}\right)=-\widetilde{c}_{1,1}+\widetilde{c}_{1,2}-\widetilde{c}_{1,3}=-\omega_{2}+\omega_{3}-\omega_{4} \\
& \partial\left(\widetilde{c}_{2,2}\right)=-\widetilde{c}_{1,1}-\widetilde{c}_{1,3}+\widetilde{c}_{1,4}=2 \omega_{1}-\omega_{3}-\omega_{4} \\
& \partial\left(\widetilde{c}_{2,3}\right)=-\widetilde{c}_{1,2}+\widetilde{c}_{1,3}-\widetilde{c}_{1,4}=-\omega_{3}+\omega_{4} \\
& \partial\left(\widetilde{c}_{2,3}\right)=\widetilde{c}_{1,1}-\widetilde{c}_{1,2}-\widetilde{c}_{1,4}=-\omega_{1}+\omega_{2}-\omega_{3}
\end{aligned}
$$

De fato,

$$
\left[\partial_{2}\right]=\left(\begin{array}{cccc}
-1 & 1 & -1 & 0 \\
-1 & 0 & -1 & 1 \\
0 & -1 & 1 & -1 \\
1 & -1 & 0 & -1
\end{array}\right) \sim\left(\begin{array}{cccc}
0 & -1 & 1 & -1 \\
2 & -1 & 0 & -1 \\
0 & 0 & -1 & 1 \\
-1 & 1 & -1 & 0
\end{array}\right)
$$

Logo,

$$
H_{1}\left(\mathscr{P}_{3} ; \mathbb{Z}_{p}\right)=\frac{\mathbb{Z}_{p}\left[\omega, \omega_{2}, \omega_{3}, \omega_{4}\right]}{\mathbb{Z}_{p}\left[-\omega_{2}+\omega_{3}-\omega_{4}, 2 \omega_{1}-\omega_{3}-\omega_{4},-\omega_{3}+\omega_{4},-\omega_{1}+\omega_{2}-\omega_{3}\right]} .
$$


Agora, fazendo a mudança de base de $\left\{\widetilde{c}_{2,1}, \widetilde{c}_{2,2}, \widetilde{c}_{2,3}, \widetilde{c}_{2,4}\right\}$ para $\left\{-\widetilde{c}_{2,1}+\widetilde{c}_{2,2},-\widetilde{c}_{2,1}-\widetilde{c}_{2,3}, \widetilde{c}_{2,3},-\widetilde{c}_{2,1}-\widetilde{c}_{2,4}\right\}=\left\{\psi_{1}, \psi_{2}, \psi_{3}, \psi_{4}\right\}$, obtemos

$$
\left(\begin{array}{cccc}
-1 & 1 & 0 & 0 \\
-1 & 0 & -1 & 0 \\
0 & 0 & 1 & 0 \\
-1 & 0 & 0 & -1
\end{array}\right) \simeq\left(\begin{array}{cccc}
-1 & 0 & -1 & 0 \\
-1 & 1 & 0 & 0 \\
0 & 0 & 1 & 0 \\
-1 & 0 & 0 & -1
\end{array}\right)
$$

Notemos que a matriz tem determinante 1.

Assim,

$$
\begin{aligned}
& \partial\left(\psi_{1}\right)=\partial\left(-\widetilde{c}_{2,1}+\widetilde{c}_{2,2}\right)=2 \omega_{1}-\omega_{3} \\
& \partial\left(\psi_{2}\right)=\partial\left(-\widetilde{c}_{2,1}-\widetilde{c}_{2,3}\right)=\omega_{2} \\
& \partial\left(\psi_{3}\right)=\partial\left(\widetilde{c}_{2,3}\right)=-\omega_{3}+\omega_{4} \\
& \partial\left(\psi_{4}\right)=\partial\left(-\widetilde{c}_{2,1}-\widetilde{c}_{2,4}\right)=\omega_{1}+\omega_{4} .
\end{aligned}
$$

Logo,

$$
H_{1}\left(\mathscr{P}_{3} ; \mathbb{Z}_{p}\right)=\frac{\mathbb{Z}_{p}\left[\omega_{1}, \omega_{2}, \omega_{3}, \omega_{4}\right]}{\mathbb{Z}_{p}\left[2 \omega_{1}-\omega_{3}, \omega_{2},-\omega_{3}+\omega_{4}, \omega_{1}+\omega_{4}\right]} \simeq \frac{\mathbb{Z}_{p}\left[\omega_{1}, \omega_{3}, \omega_{4}\right]}{\mathbb{Z}_{p}\left[2 \omega_{1}-\omega_{3},-\omega_{3}+\omega_{4}, \omega_{1}+\omega_{4}\right]}
$$

Mudando a base de $\left\{\psi_{1}, \psi_{3}, \psi_{4}\right\}$ para $\left\{\psi_{1}-\psi_{3}+\psi_{4}, \psi_{3}, \psi_{4}\right\}=\left\{\sigma_{1}, \sigma_{2}, \sigma_{3}\right\}$, obtemos a matriz

$$
\left(\begin{array}{ccc}
1 & -1 & 1 \\
0 & 1 & 0 \\
0 & 0 & 1
\end{array}\right)
$$

que tem determinante 1. Desse modo,

$$
\begin{aligned}
& \partial\left(\sigma_{1}\right)=\partial\left(\psi_{1}-\psi_{3}+\psi_{4}\right)=3 \omega_{1} \\
& \partial\left(\sigma_{2}\right)=\partial\left(\psi_{3}\right)=-\omega_{3}+\omega_{4} \\
& \partial\left(\sigma_{3}\right)=\partial\left(\psi_{4}\right)=\omega_{1}+\omega_{4} .
\end{aligned}
$$

Assim,

$$
H_{1}\left(\mathscr{P}_{3} ; \mathbb{Z}_{p}\right)=\frac{\mathbb{Z}_{p}\left[\omega_{1}, \omega_{2}, \omega_{3}\right]}{\mathbb{Z}_{p}\left[3 \omega_{1},-\omega_{3}+\omega_{4}, \omega_{1}+\omega_{4}\right]}
$$


Por fim, fazendo a mudança de base de $\left\{\omega_{1}, \omega_{3}, \omega_{4}\right\}$ para $\left\{\omega_{1},-\omega_{3}+\omega_{4}, \omega_{1}+\omega_{4}\right\}=$ $=\left\{\delta_{1}, \delta_{2}, \delta_{3}\right\}$, temos

$$
\left(\begin{array}{ccc}
1 & 0 & 0 \\
0 & -1 & 1 \\
1 & 0 & 1
\end{array}\right)
$$

A matriz mudança de base possui determinante -1 .

Logo,

$$
\begin{aligned}
& \partial\left(\sigma_{1}\right)=3 \omega_{1}=3 \delta_{1} \\
& \partial\left(\sigma_{2}\right)=-\omega_{3}+\omega_{4}=\delta_{2} \\
& \partial\left(\sigma_{3}\right)=\omega_{1}+\omega_{4}=\delta_{3} .
\end{aligned}
$$

Então,

$$
H_{1}\left(\mathscr{P}_{3} ; \mathbb{Z}_{p}\right)=\frac{\mathbb{Z}_{p}\left[\delta_{1}, \delta_{2}, \delta_{3}\right]}{\mathbb{Z}_{p}\left[3 \delta_{1}, \delta_{2}, \delta_{3}\right]} \simeq \frac{\mathbb{Z}_{p}\left[\delta_{1}\right]}{\mathbb{Z}_{p}\left[3 \delta_{1}\right]} \simeq \frac{\mathbb{Z}_{p}\left[\delta_{1}\right]}{\mathbb{Z}_{p}\left[\delta_{1}\right]} \simeq 0
$$

- $H_{2}\left(\mathscr{P}_{3} ; \mathbb{Z}_{p}\right)$

Como $\left[\partial_{3}\right]=\left(\begin{array}{cccc}0 & 0 & 0 & 0\end{array}\right)$ temos $\operatorname{Im}_{3}=\{0\}$. Além disso,

$$
\left[\partial_{2}\right]=\left(\begin{array}{cccc}
-1 & 1 & -1 & 0 \\
-1 & 0 & -1 & 1 \\
0 & -1 & 1 & -1 \\
1 & -1 & 0 & -1
\end{array}\right)
$$

possui o determinante não nulo, portanto $\partial_{2}$ é injetor, ou seja, $\operatorname{Ker} \partial_{2}=\{0\}$. Assim,

$$
H_{2}\left(\mathscr{P}_{3} ; \mathbb{Z}_{p}\right)=\frac{\operatorname{Ker} \partial_{2}}{\operatorname{Im} \partial_{3}}=\frac{\{0\}}{\{0\}}=0
$$

- $H_{3}\left(\mathscr{P}_{3} ; \mathbb{Z}_{p}\right)$

$$
H_{3}\left(\mathscr{P}_{3} ; \mathbb{Z}_{p}\right)=\frac{\operatorname{Ker} \partial_{3}}{\operatorname{Im} \partial_{4}}=\frac{\mathbb{Z}_{p}}{\{0\}}=\mathbb{Z}_{p}, \text { pois }\left[\partial_{3}\right]=\left(\begin{array}{ccc}
0 & 0 & 0
\end{array}\right) .
$$


Portanto,

$$
H_{i}\left(\mathscr{P}_{3} ; \mathbb{Z}_{p}\right)= \begin{cases}\mathbb{Z}_{p}, & \mathrm{i}=0,3 \\ 0, & \mathrm{i}=1,2\end{cases}
$$

\subsection{Grupos de homologia de $\mathscr{P}_{4 n-1}, n \geq 1$}

Calculemos os grupos de homologia de $\mathscr{P}_{4 n-1}=\frac{S^{4 n-1}}{\eta\left(P_{24}\right)}$, com representação $\eta: P_{24} \rightarrow A u t(\mathbb{Z})$ tal que $\eta:(x, y, z) \rightarrow(1,1,1)$. Tal representação é única, pela mesma justificativa utilizada no início da seção anterior.

Para as subseções a seguir, consideremos o complexo de cadeias abaixo:

$$
\begin{gathered}
\cdots \longrightarrow \mathbb{Z} P_{24}\left[\widetilde{c}_{4 q-1,1}\right] \stackrel{\partial_{4 q-1}}{\longrightarrow} \mathbb{Z} P_{24}\left[\widetilde{c}_{4 q-2,1}, \widetilde{c}_{4 q-2,2}, \widetilde{c}_{4 q-2,3}, \widetilde{c}_{4 q-2,4}\right] \longrightarrow \\
\stackrel{\partial_{4 q-2}}{\longrightarrow} \mathbb{Z} P_{24}\left[\widetilde{c}_{4 q-3,1}, \widetilde{c}_{4 q-3,2}, \widetilde{c}_{4 q-3,3}, \widetilde{c}_{4 q-3,4}\right] \stackrel{\partial_{4 q-3}}{\longrightarrow} \mathbb{Z} P_{24}\left[\widetilde{c}_{4 q-4,1}\right] \stackrel{\partial_{4 q-4}}{\longrightarrow} \cdots .
\end{gathered}
$$

\subsubsection{Grupos de homologia com coeficientes em $\mathbb{Z}$}

Seja $\eta:(x, y, z) \rightarrow(1,1,1)$ a representação trivial. Os operadores bordo são dados por

$$
\begin{gathered}
{\left[\partial_{4 q-1}\right]=\left(\begin{array}{llll}
0 & 0 & 0 & 0
\end{array}\right),} \\
{\left[\partial_{4 q-2}\right]=\left(\begin{array}{cccc}
-1 & 1 & -1 & 0 \\
-1 & 0 & -1 & 1 \\
0 & -1 & 1 & -1 \\
1 & -1 & 0 & -1
\end{array}\right),} \\
{\left[\partial_{4 q-3}\right]=\left(\begin{array}{c}
0 \\
0 \\
0 \\
0
\end{array}\right)} \\
{\left[\partial_{4 q-4}\right]=\left(\begin{array}{l}
24
\end{array}\right)}
\end{gathered}
$$


exceto por $\partial_{0}=0$.

De maneira análoga ao cálculo da homologia de $\mathscr{P}_{3}$, obtemos

$$
\begin{gathered}
H_{4 n-1}\left(\mathscr{P}_{4 n-1} ; \mathbb{Z}\right) \simeq H_{0}\left(\mathscr{P}_{4 n-1} ; \mathbb{Z}\right) \simeq \mathbb{Z} \\
H_{4 q-2}\left(\mathscr{P}_{4 n-1} ; \mathbb{Z}\right) \simeq 0 \\
H_{4 q-3}\left(\mathscr{P}_{4 n-1} ; \mathbb{Z}\right) \simeq \mathbb{Z}_{3} .
\end{gathered}
$$

Resta calcular $H_{4 q-4}\left(\mathscr{P}_{4 n-1} ; \mathbb{Z}\right)$ e $H_{4 q-1}\left(\mathscr{P}_{4 n-1} ; \mathbb{Z}\right)$.

- $H_{4 q-4}\left(\mathscr{P}_{4 n-1} ; \mathbb{Z}\right)$

Analisemos o Ker $\partial_{4 q-4}$. Seja $\sigma$ uma $(4 q-4)$-cadeia representada por $a$.

$$
\partial_{4 q-4}(\sigma)=24 a=0 \Longleftrightarrow a=0 .
$$

Logo, $\operatorname{Ker} \partial_{4 q-4}=\{0\}$.

Assim,

$$
H_{4 q-4}\left(\mathscr{P}_{4 n-1} ; \mathbb{Z}\right)=\frac{\operatorname{Ker} \partial_{4 q-4}}{\operatorname{Im} \partial_{4 q-3}}=\frac{\{0\}}{\{0\}}=0
$$

- $H_{4 q-1}\left(\mathscr{P}_{4 n-1} ; \mathbb{Z}\right)$

O Ker $\partial_{4 q-1} \simeq \mathbb{Z}$, uma vez que $\partial_{4 q-1}$ é a aplicação nula.

Além disso, notemos que $\operatorname{Im} \partial_{4 q-4} \simeq 24 \mathbb{Z}$.

Dessa forma,

$$
H_{4 q-1}\left(\mathscr{P}_{4 n-1} ; \mathbb{Z}\right)=\frac{\operatorname{Ker} \partial_{4 q-1}}{\operatorname{Im} \partial_{4 q-4}}=\frac{\mathbb{Z}}{24 \mathbb{Z}}=\mathbb{Z}_{24}
$$

\subsubsection{Grupos de homologia com coeficientes em $\mathbb{Z}_{2}$}

Seja $\eta:(x, y, z) \rightarrow(1,1,1)$ a representação trivial. Os operadores bordo são dados por

$$
\left[\partial_{4 q-1}\right]=\left(\begin{array}{llll}
0 & 0 & 0 & 0
\end{array}\right)
$$




$$
\begin{gathered}
{\left[\partial_{4 q-2}\right]=\left(\begin{array}{cccc}
-1 & 1 & -1 & 0 \\
-1 & 0 & -1 & 1 \\
0 & -1 & 1 & -1 \\
1 & -1 & 0 & -1
\end{array}\right)} \\
{\left[\partial_{4 q-3}\right]=\left(\begin{array}{c}
0 \\
0 \\
0 \\
0
\end{array}\right)} \\
{\left[\partial_{4 q-4}\right]=(24)=(0)}
\end{gathered}
$$

exceto por $\partial_{0}=0$.

Analogamente ao cálculo da homologia de $\mathscr{P}_{3}$, obtemos

$$
\begin{gathered}
H_{4 n-1}\left(\mathscr{P}_{4 n-1} ; \mathbb{Z}_{2}\right) \simeq H_{0}\left(\mathscr{P}_{4 n-1} ; \mathbb{Z}_{2}\right) \simeq \mathbb{Z}_{2} \\
H_{4 q-2}\left(\mathscr{P}_{4 n-1} ; \mathbb{Z}_{2}\right) \simeq 0 \\
H_{4 q-3}\left(\mathscr{P}_{4 n-1} ; \mathbb{Z}_{2}\right) \simeq 0 .
\end{gathered}
$$

Resta encontrar $H_{4 q-4}\left(\mathscr{P}_{4 n-1} ; \mathbb{Z}_{2}\right)$ e $H_{4 q-1}\left(\mathscr{P}_{4 n-1} ; \mathbb{Z}_{2}\right)$ :

- $H_{4 q-4}\left(\mathscr{P}_{4 n-1} ; \mathbb{Z}_{2}\right)$

Como $\partial_{4 q-4}$ é a aplicalção nula, temos $\operatorname{Ker} \partial_{4 q-4} \simeq \mathbb{Z}_{2}$.

Portanto,

$$
H_{4 q-4}\left(\mathscr{P}_{4 n-1} ; \mathbb{Z}_{2}\right)=\frac{\operatorname{Ker} \partial_{4 q-4}}{\operatorname{Im} \partial_{4 q-3}}=\frac{\mathbb{Z}_{2}}{\{0\}}=\mathbb{Z}_{2}
$$

- $H_{4 q-1}\left(\mathscr{P}_{4 n-1} ; \mathbb{Z}_{2}\right)$

O Ker $\partial_{4 q-1} \simeq \mathbb{Z}_{2}$, uma vez que $\partial_{4 q-1}$ é a aplicação nula.

Além disso, temos $\operatorname{Im} \partial_{4 q-4} \simeq\{0\}$.

Dessa forma,

$$
H_{4 q-1}\left(\mathscr{P}_{4 n-1} ; \mathbb{Z}_{2}\right)=\frac{\operatorname{Ker} \partial_{4 q-1}}{\operatorname{Im} \partial_{4 q-4}}=\frac{\mathbb{Z}_{2}}{\{0\}}=\mathbb{Z}_{2}
$$




\subsubsection{Grupos de homologia com coeficientes em $\mathbb{Z}_{3}$}

Consideremos $\eta:(x, y, z) \rightarrow(1,1,1)$ a representação trivial. Os operadores bordo são dados por

$$
\begin{gathered}
{\left[\partial_{4 q-1}\right]=\left(\begin{array}{cccc}
0 & 0 & 0 & 0
\end{array}\right)} \\
{\left[\partial_{4 q-2}\right]=\left(\begin{array}{cccc}
-1 & 1 & -1 & 0 \\
-1 & 0 & -1 & 1 \\
0 & -1 & 1 & -1 \\
1 & -1 & 0 & -1
\end{array}\right),} \\
{\left[\partial_{4 q-3}\right]=\left(\begin{array}{c}
0 \\
0 \\
0 \\
0
\end{array}\right)} \\
{\left[\partial_{4 q-4}\right]=(24)=(0)}
\end{gathered}
$$

exceto por $\partial_{0}=0$.

De modo similar ao cálculo da homologia de $\mathscr{P}_{3}$, obtemos

$$
\begin{gathered}
H_{4 n-1}\left(\mathscr{P}_{4 n-1} ; \mathbb{Z}_{3}\right) \simeq H_{0}\left(\mathscr{P}_{4 n-1} ; \mathbb{Z}_{3}\right) \simeq \mathbb{Z}_{3}, \\
H_{4 q-2}\left(\mathscr{P}_{4 n-1} ; \mathbb{Z}_{3}\right) \simeq \mathbb{Z}_{3}, \\
H_{4 q-3}\left(\mathscr{P}_{4 n-1} ; \mathbb{Z}_{3}\right) \simeq \mathbb{Z}_{3} .
\end{gathered}
$$

Falta calcular $H_{4 q-4}\left(\mathscr{P}_{4 n-1} ; \mathbb{Z}_{3}\right)$ e $H_{4 q-1}\left(\mathscr{P}_{4 n-1} ; \mathbb{Z}_{3}\right)$ :

- $H_{4 q-4}\left(\mathscr{P}_{4 n-1} ; \mathbb{Z}_{3}\right)$

Como $\partial_{4 q-4}$ é a aplicalção nula, temos $\operatorname{Ker} \partial_{4 q-4} \simeq \mathbb{Z}_{3}$.

Portanto,

$$
H_{4 q-4}\left(\mathscr{P}_{4 n-1} ; \mathbb{Z}_{3}\right)=\frac{K e r \partial_{4 q-4}}{\operatorname{Im} \partial_{4 q-3}}=\frac{\mathbb{Z}_{3}}{\{0\}}=\mathbb{Z}_{3}
$$


- $H_{4 q-1}\left(\mathscr{P}_{4 n-1} ; \mathbb{Z}_{3}\right)$

O Ker $\partial_{4 q-1} \simeq \mathbb{Z}_{3}$, uma vez que $\partial_{4 q-1}$ é a aplicação nula.

Além disso, notemos que $\operatorname{Im} \partial_{4 q-4} \simeq\{0\}$.

Dessa forma,

$$
H_{4 q-1}\left(\mathscr{P}_{4 n-1} ; \mathbb{Z}_{2}\right)=\frac{\operatorname{Ker} \partial_{4 q-1}}{\operatorname{Im} \partial_{4 q-4}}=\frac{\mathbb{Z}_{3}}{\{0\}}=\mathbb{Z}_{3} .
$$

\subsubsection{Grupos de homologia com coeficientes em $\mathbb{Z}_{p}$, com $p$ primo e $p>3$}

Consideremos a representação trivial $\eta:(x, y, z) \rightarrow(1,1,1)$. Os operadores bordo são dados por

$$
\begin{gathered}
{\left[\partial_{4 q-1}\right]=\left(\begin{array}{cccc}
0 & 0 & 0 & 0
\end{array}\right)} \\
{\left[\partial_{4 q-2}\right]=\left(\begin{array}{cccc}
-1 & 1 & -1 & 0 \\
-1 & 0 & -1 & 1 \\
0 & -1 & 1 & -1 \\
1 & -1 & 0 & -1
\end{array}\right)} \\
{\left[\partial_{4 q-3}\right]=\left(\begin{array}{c}
0 \\
0 \\
0 \\
0
\end{array}\right)} \\
{\left[\partial_{4 q-4}\right]=\left(\begin{array}{l}
24
\end{array}\right)}
\end{gathered}
$$

exceto por $\partial_{0}=0$.

De forma análoga ao cálculo da homologia de $\mathscr{P}_{3}$, obtemos

$$
\begin{gathered}
H_{4 n-1}\left(\mathscr{P}_{4 n-1} ; \mathbb{Z}_{p}\right) \simeq H_{0}\left(\mathcal{P} ; \mathbb{Z}_{p}\right) \simeq \mathbb{Z}_{p} \\
H_{4 q-2}\left(\mathscr{P}_{4 n-1} ; \mathbb{Z}_{p}\right) \simeq 0 \\
H_{4 q-3}\left(\mathscr{P}_{4 n-1} ; \mathbb{Z}_{p}\right) \simeq 0 .
\end{gathered}
$$


Resta calcular $H_{4 q-4}\left(\mathscr{P}_{4 n-1} ; \mathbb{Z}_{p}\right)$ e $H_{4 q-1}\left(\mathscr{P}_{4 n-1} ; \mathbb{Z}_{p}\right)$ :

- $H_{4 q-4}\left(\mathscr{P}_{4 n-1} ; \mathbb{Z}_{p}\right)$

Analisemos o Ker $\partial_{4 q-4}$. Seja $\sigma$ uma $(4 q-4)$-cadeia representada por $a$.

$$
\partial_{4 q-4}(\sigma)=24 a=0 \Longleftrightarrow a=0 .
$$

Logo, $\operatorname{Ker} \partial_{4 q-4}=\{0\}$.

Portanto,

$$
H_{4 q-4}\left(\mathscr{P}_{4 n-1} ; \mathbb{Z}_{p}\right)=\frac{\operatorname{Ker} \partial_{4 q-4}}{\operatorname{Im} \partial_{4 q-3}}=\frac{\{0\}}{\{0\}}=.0
$$

- $H_{4 q-1}\left(\mathscr{P}_{4 n-1} ; \mathbb{Z}_{p}\right)$

O Ker $\partial_{4 q-1} \simeq \mathbb{Z}_{p}$, uma vez que $\partial_{4 q-1}$ é a aplicação nula.

Além disso, $\operatorname{Im} \partial_{4 q-4} \simeq 24 \mathbb{Z}_{p}$.

Dessa forma,

$$
H_{4 q-1}\left(\mathscr{P}_{4 n-1} ; \mathbb{Z}_{p}\right)=\frac{\operatorname{Ker} \partial_{4 q-1}}{\operatorname{Im} \partial_{4 q-4}}=\frac{\mathbb{Z}_{p}}{24 \mathbb{Z}_{p}}=\mathbb{Z}_{24 p}
$$


Capítulo

4

\section{Anel de cohomologia das formas espaciais esféricas tetraedrais}

Apresentamos, inicialmente, alguns resultados necessários para uma melhor compreensão do capítulo, como: o Teorema dos Coeficientes Universais para cohomologia, a definição de produto cup e sua interpretação geométrica, entre outros. Utilizamos os complexos de cadeias construídos nas Seções 2.2 e 2.3, assim como os grupos de homologia, calculados anteriormente, para encontrar os grupos de cohomologia (com coeficientes locais) da forma espacial esférica tetraedral de dimensão três com coeficientes em $\mathbb{Z}, \mathbb{Z}_{2}, \mathbb{Z}_{3}$ e $\mathbb{Z}_{p}, p$ primo e $p>3$. Assim, através da interpretação geométrica do produto cup, calculamos o anel de cohomologia desse espaço, que é o objetivo principal do capítulo. Por fim, encontramos a homotopia contrátil para as formas espaciais esféricas tetraedrais, a qual será responsável, em trabalhos futuros, pelo cálculo do anel de cohomologia para tais espaços em dimensão $4 n-1, n \geq 1$. Os pré-requisitos para o capítulo são baseados em [3], [4], [14], [18] e [22].

\subsection{Módulo de homomorfismos}

Sejam $A$ e $B, R$-módulos à esquerda arbitrários e consideremos

$$
\Phi=H_{\mathrm{om}}(A, B)
$$


o conjunto de todos os homomorfismos do módulo $A$ no módulo $B$.

Quando não houver problema de ambiguidade, como é o nosso caso, já que estamos trabalhando com módulos sobre um anel $R$ fixado, denotaremos simplesmente

$$
\Phi=\operatorname{Hom}(A, B)
$$

Definamos uma adição $(+)$ no conjunto $\Phi$ do seguinte modo: para dois homomorfismos $\phi, \psi: A \rightarrow B$, o homomorfismo

$$
\phi+\psi: A \rightarrow B
$$

é definido por $(\phi+\psi)(x)=\phi(x)+\psi(x)$, para todo $x$ pertencente a $A$.

Esta adição $(+)$ torna $\Phi$ um grupo abeliano.

Agora, para todo $\lambda$ pertencente a $R$ e para todo $\phi$ pertencente a $\Phi$, consideremos a função

$$
\lambda \phi: A \rightarrow B
$$

definida por $(\lambda \phi)(x)=\lambda[\phi(x)]$, para todo $x$ pertencente a $A$.

Podemos verificar que $\lambda \phi$ é um homomorfismo do módulo $A$ no módulo $B$ e a aplicação $(\lambda, \phi) \mapsto \lambda \phi$ define uma multiplicação escalar

$$
\mu: R \times \Phi \rightarrow \Phi
$$

no grupo abeliano $\Phi$.

Isto torna $\Phi$ um $R$-módulo à esquerda, sendo o elemento neutro de $\Phi$ o homomorfismo trivial 0 .

Definição 4.1.1 O R-módulo $\Phi=\operatorname{Hom}(A, B)$ é chamado módulo de homomorfismos do módulo A no módulo $B$.

Proposição 4.1.2 ([14], 1.8.1) Para qualquer $R$-módulo $X$ à esquerda, sempre temos

$$
\operatorname{Hom}(R, X) \simeq X
$$

Sejam $f: A^{\prime} \rightarrow A$ e $g: B \rightarrow B^{\prime}$ homomorfismos de $R$-módulo à esquerda e consideremos 
os módulos $\operatorname{Hom}(A, B)$ e $\operatorname{Hom}\left(A^{\prime}, B^{\prime}\right)$. Definamos uma função

$h: \operatorname{Hom}(A, B) \rightarrow \operatorname{Hom}\left(A^{\prime}, B^{\prime}\right)$ tal que $h(\phi)=g \circ \phi \circ f$, para todo $\phi \in \operatorname{Hom}(A, B)$.

Esta função $h$ é um homomorfismo do módulo $\operatorname{Hom}(A, B)$ no módulo $\operatorname{Hom}\left(A^{\prime}, B^{\prime}\right)$, a qual denotamos por $\operatorname{Hom}(f, g)$. Se $g=i d$, denotaremos simplesmente por

$$
\operatorname{Hom}(f): \operatorname{Hom}(A, B) \rightarrow \operatorname{Hom}\left(A^{\prime}, B^{\prime}\right) .
$$

Proposição 4.1.3 ([14], 1.8.2) Se $i: A \rightarrow A$ e $j: B \rightarrow B$ são homomorfismos idênticos dos $R$-módulos à esquerda $A$ e $B$, então

$$
\operatorname{Hom}(i, j): \operatorname{Hom}(A, B) \rightarrow \operatorname{Hom}(A, B)
$$

é o homomorfismo idêntico do módulo $\operatorname{Hom}(A, B)$. Se $f: A^{\prime} \rightarrow A, f^{\prime}: A^{\prime \prime} \rightarrow A^{\prime}$, $g: B \rightarrow B^{\prime}, g^{\prime}: B^{\prime} \rightarrow B^{\prime \prime}$ são homomorfismos de $R$-módulos à esquerda, então

$$
\operatorname{Hom}\left(f \circ f^{\prime}, g^{\prime} \circ g\right)=\operatorname{Hom}\left(f^{\prime}, g^{\prime}\right) \circ \operatorname{Hom}(f, g) \text {. }
$$

Corolário 4.1.4 ([14], 1.8.3) Se $f: A^{\prime} \rightarrow A$ e $g: B \rightarrow B^{\prime}$ são isomorfismos de $R$-módulo $\grave{a}$ esquerda, então $\operatorname{Hom}(f, g): \operatorname{Hom}(A, B) \rightarrow \operatorname{Hom}\left(A^{\prime}, B^{\prime}\right)$ também é isomorfismo.

Definição 4.1.5 Seja A um grupo abeliano e $F(A)$ o grupo abeliano livre gerado pelos elementos de $A$. Considere $R(A)$ o kernel da projeção natural $F(A) \rightarrow A$. A sequência

$$
0 \longrightarrow R(A) \stackrel{\phi}{\longrightarrow} F(A) \longrightarrow A \longrightarrow 0
$$

é chamada de resolução canônica livre de A. Seja $\widetilde{\phi}=\operatorname{Hom}(\phi)$. O grupo

$$
\operatorname{cokernel}(\widetilde{\phi})=\operatorname{Hom}(R(A), B) / \widetilde{\phi}(\operatorname{Hom}(F(A), B))
$$

é denotado por $\operatorname{Ext}(A, B)$. 


\section{Teorema 4.1.6 ([18], Teorema 53.1, dos Coeficientes Universais para cohomologia)}

Se $C$ é um complexo de cadeias livre e $G$ é um grupo abeliano, então existe uma sequência exata

$$
0 \longleftarrow \operatorname{Hom}\left(H_{p}(C), G\right) \stackrel{k}{\longleftarrow} H^{p}(C ; G) \longleftarrow \operatorname{Ext}\left(H_{p-1}(C), G\right) \longleftarrow 0
$$

onde as aplicações são os homomorfismos naturais induzidos pelo complexo de cadeias e a sequência acima cinde. Logo,

$$
H^{p}(C ; G) \simeq \operatorname{Hom}\left(H_{p}(C), G\right) \oplus \operatorname{Ext}\left(H_{p-1}(C), G\right) .
$$

Em [18, Seção 54] temos algumas regras para calcular Hom e Ext quando os grupos são finitamente gerados. A tabela abaixo apresenta as equivalências que utilizamos neste trabalho. Considere, na tabela, $d=m d c(m, n)$.

\begin{tabular}{|c|c|}
\hline $\operatorname{Hom}(\mathbb{Z}, G) \simeq G$ & $\operatorname{Ext}(\mathbb{Z}, G)=0$ \\
\hline $\operatorname{Hom}(\mathbb{Z} / m, G) \simeq \operatorname{Ker}(G \stackrel{m}{\rightarrow} G)$ & $\operatorname{Ext}(\mathbb{Z} / m, G) \simeq G / m G$ \\
\hline $\operatorname{Hom}(\mathbb{Z} / m, \mathbb{Z})=0$ & $\operatorname{Ext}(\mathbb{Z} / m, \mathbb{Z}) \simeq \mathbb{Z} / m$ \\
\hline $\operatorname{Hom}(\mathbb{Z} / m, \mathbb{Z} / n) \simeq \mathbb{Z} / d$ & $\operatorname{Ext}(\mathbb{Z} / m, \mathbb{Z} / n) \simeq \mathbb{Z} / d$ \\
\hline
\end{tabular}

\subsection{Produto Cup}

Definição 4.2.1 Sejam $X$ um espaço topológico e $S^{p}(X ; R)=\operatorname{Hom}\left(S_{p}(X ; R)\right)$ o grupo das p-cocadeias singulares de $X$, com coeficientes em $R$. Definimos uma aplicação

$$
S^{p}(X ; R) \times S^{q}(X ; R) \stackrel{\cup}{\longrightarrow} S^{p+q}(X ; R)
$$

da seguinte maneira: se $T: \Delta_{p+q} \rightarrow X$ é um $p+q$-simplexo singular, seja

$$
\left(c^{p} \cup c^{q}\right)(T)=c^{p}\left(T \circ l\left(\epsilon_{0}, \ldots, \epsilon_{p}\right)\right) \cdot c^{q}\left(T \circ l\left(\epsilon_{p}, \ldots, \epsilon_{p+q}\right)\right) .
$$

A cocadeia $c^{p} \cup c^{q}$ é chamada de produto cup das cocadeias $c^{p}$ e $c^{q}$.

A aplicação $T \circ l\left(\epsilon_{0}, \ldots, \epsilon_{p}\right)$ é apenas a restrição de $T$ à $p$-face $\Delta_{p}$ de $\Delta_{p+q}$, que é um 
$p$-simplexo singular em $X$. Similarmente, $T \circ l\left(\epsilon_{p}, \ldots, \epsilon_{p+q}\right)$ é a restrição de $T$ à $q$-face de $\Delta_{p+q}$, que é um $q$-simplexo singular em $X$. A multiplicação indicada no lado direito da igualdade é a multiplicação no anel $R$.

Definição 4.2.2 Seja $H^{*}(X ; R)$ a soma direta $\bigoplus H^{i}(X ; R)$. A operação produto cup torna esse grupo um anel com um elemento unitário. Esse anel é chamado anel de cohomologia de $X$ com coeficientes em $R$.

\subsubsection{Interpretação geométrica}

O objetivo desta seção é apresentar uma interpretação geométrica para o produto cup. Primeiramente, recordemos o Teorema da Dualidade de Poincaré.

Teorema 4.2.3 ([18], Teorema 65.1, da dualidade de Poincaré) Seja $X \quad$ uma variedade n-dimensional compacta, fechada e orientável, então para todo $p$, existe um isomorfismo

$$
H^{p}(X ; G) \simeq H_{n-p}(X ; G),
$$

onde $G$ é um grupo com coeficientes arbitrários. Se $X$ é não-orientável, existe um isomorfismo

$$
H^{p}\left(X ; \mathbb{Z}_{2}\right) \simeq H_{n-p}\left(X ; \mathbb{Z}_{2}\right)
$$

para todo $p$.

Se $X$ é uma variedade suave fechada e orientável, e se $A$ e $B$ são subvariedades orientadas de $X$, que se interceptam transversalmente, então o produto cup do dual de Poincaré de $A$ e $B$ é o dual de Poincaré de $A \cap B$, ou seja, o produto cup é o dual de Poincaré da intersecção das subvariedades.

Seja $X$ uma variedade suave fechada e orientável de dimensão $n$. Sejam $A$ e $B$ subvariedades suaves fechadas e orientáveis de dimensão $n-i$ e $n-j$, respectivamente. Suponha que $A$ e $B$ se interceptam transversalmente. Isso significa que para todo $p$ pertencente a $A \cap B$, a aplicação

$$
T_{p} A \oplus T_{p} B \rightarrow T_{p} X
$$


induzida pelas inclusões, é sobrejetora. Essa condição pode ser obtida por pequenas perturbações nos mergulhos de $A$ e $B$ em $X$. Dessa forma, $A \cap B$ é uma subvariedade de dimensão $n-(i+j)$.

Considere as classes de homologia $[A] \in H_{n-i}(X ; \mathbb{Z}),[B] \in H_{n-j}(X ; \mathbb{Z})$ e $[A \cap B]$ $\in H_{n-i-j}(X ; \mathbb{Z})$. Denotamos o dual de Poincaré de cada classe por $[A]^{*} \in H^{i}(X ; \mathbb{Z}),[B]^{*}$ $\in H^{j}(X ; \mathbb{Z})$ e $[A \cap B]^{*} \in H^{i+j}(X ; \mathbb{Z})$.

Nas condições acima, enunciamos o seguinte teorema:

Teorema 4.2 .4 ([3], VI.11) O produto cup do dual de Poincaré de $A$ e $B$ é o dual de Poincaré da intersecção:

$$
[A]^{*} \cup[B]^{*}=[A \cap B]^{*} \in H^{i+j}(X ; \mathbb{Z}) .
$$

Este teorema só responde parcialmente qual o significado geométrico do produto cup pois, só funciona para uma variedade suave e, além disso, nem toda classe de homologia em uma variedade pode ser representada por uma subvariedade. No entanto, este teorema é muito útil.

De maneira análoga, existe uma versão do teorema anterior para variedades não orientáveis, usando $\mathbb{Z}_{2}$ como coeficientes.

Exemplo 4.2.5 Seja $X=T^{2}=\mathbb{R}^{2} / \mathbb{Z}^{2}$. Dessa forma, $H_{1}\left(T^{2}\right) \simeq \mathbb{Z}^{2}=\mathbb{Z} \oplus \mathbb{Z}$, com geradores $[A]$ e $[B]$, e $H_{0}\left(T^{2}\right) \simeq \mathbb{Z}$, com gerador $[p]$.

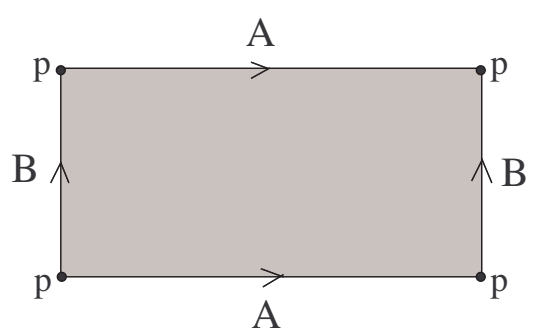

Sejam $[A]^{*},[B]^{*} \in H^{1}\left(T^{2} ; \mathbb{Z}\right)$ e $[p]^{*} \in H^{2}\left(T^{2} ; \mathbb{Z}\right)$ o dual de Poincaré de $[A],[B]$ e $[p]$, respectivamente. Considerando positiva a orientação anti-horário, segue que $A \cap B=p$ é orientada positivamente e $B \cap A=-p$ é orientada negativamente. Assim, 
pelo Teorema 4.2.4, temos

$$
[A]^{*} \cup[B]^{*}=[A \cap B]^{*}=[p]^{*} \text { e }[B]^{*} \cup[A]^{*}=[B \cap A]^{*}=[-p]^{*}=-[p]^{*} .
$$

Por outro lado,

$$
[A]^{*} \cup[A]^{*}=[B]^{*} \cup[B]^{*}=0 .
$$

Nas condições do Teorema 4.2.4 para calcular $[A]^{*} \cup[A]^{*}$, precisamos calcular o número de intersecção de duas subvariedades $A_{1}$ e $A_{2}$, que representam a mesma classe $[A]$ e se interceptam transversalmente. Não podemos escolher $A_{1}=A_{2}$ mas, podemos escolher $A_{1} e$ $A_{2}$ "paralelos", como nesse caso, logo $A_{1}$ e $A_{2}$ não se interceptam.

\subsection{Grupos de cohomologia de $\mathscr{P}_{3}$}

Consideremos o complexo de cadeias abaixo:

$C: 0 \longrightarrow \mathbb{Z} P_{24}\left[\widetilde{c}_{3,1}\right] \stackrel{\partial_{3}}{\longrightarrow} \mathbb{Z} P_{24}\left[\widetilde{c}_{2,1}, \widetilde{c}_{2,2}, \widetilde{c}_{2,3}, \widetilde{c}_{2,4}\right] \stackrel{\partial_{2}}{\longrightarrow} \mathbb{Z} P_{24}\left[\widetilde{c}_{1,1}, \widetilde{c}_{1,2}, \widetilde{c}_{1,3}, \widetilde{c}_{1,4}\right] \stackrel{\partial_{1}}{\longrightarrow} \mathbb{Z} P_{24}\left[\widetilde{c}_{0,1}\right] \longrightarrow 0$

Com relação a representação trivial $\eta:(x, y, z) \rightarrow(1,1,1)$, os operadores bordos mencionados no complexo de cadeias acima são:

$$
\begin{aligned}
& \partial_{1}\left(\widetilde{c}_{1,1}\right)=0 \\
& \partial_{1}\left(\widetilde{c}_{1,2}\right)=0 \\
& \partial_{1}\left(\widetilde{c}_{1,3}\right)=0 \\
& \partial_{1}\left(\widetilde{c}_{1,4}\right)=0 \\
& \partial_{2}\left(\widetilde{c}_{2,1}\right)=-\widetilde{c}_{1,1}+\widetilde{c}_{1,2}-\widetilde{c}_{1,3} \\
& \partial_{2}\left(\widetilde{c}_{2,2}\right)=-\widetilde{c}_{1,1}-\widetilde{c}_{1,3}+\widetilde{c}_{1,4} \\
& \partial_{2}\left(\widetilde{c}_{2,3}\right)=-\widetilde{c}_{1,2}+\widetilde{c}_{1,3}-\widetilde{c}_{1,4} \\
& \partial_{2}\left(\widetilde{c}_{2,4}\right)=\widetilde{c}_{1,1}-\widetilde{c}_{1,2}-\widetilde{c}_{1,4} \\
& \partial_{3}\left(\widetilde{c}_{3,1}\right)=0 .
\end{aligned}
$$


Nas subseções a seguir, utilizamos o complexo de cadeias, apresentado anteriormente, para calcular os grupos de cohomologia da forma espacial esférica tetraedral, de dimensão três, com coeficientes em $\mathbb{Z}, \mathbb{Z}_{2}, \mathbb{Z}_{3}$ e $\mathbb{Z}_{p}$, com $p$ primo e $p>3$.

\subsubsection{Grupos de cohomologia com coeficientes em $\mathbb{Z}$}

Calculemos a cohomologia do complexo de cadeias $C$ com coeficientes em $\mathbb{Z}$, utilizando o Teorema 4.1.6, dos Coeficientes Universias para cohomologia.

Para $q=0$, temos

$$
H^{q}(C ; \mathbb{Z}) \simeq H o m\left(H_{0}(C), \mathbb{Z}\right) \oplus \operatorname{Ext}\left(H_{-1}(C), \mathbb{Z}\right) \simeq \mathbb{Z} \oplus 0 \simeq \mathbb{Z}
$$

Para $q=1$, temos

$$
H^{q}(C ; \mathbb{Z}) \simeq \operatorname{Hom}\left(H_{1}(C), \mathbb{Z}\right) \oplus \operatorname{Ext}\left(H_{0}(C), \mathbb{Z}\right) \simeq \operatorname{Hom}\left(\mathbb{Z}_{3}, \mathbb{Z}\right) \oplus \operatorname{Ext}(\mathbb{Z}, \mathbb{Z}) \simeq 0 .
$$

Para $q=2$, temos

$H^{q}(C ; \mathbb{Z}) \simeq \operatorname{Hom}\left(H_{2}(C), \mathbb{Z}\right) \oplus \operatorname{Ext}\left(H_{1}(C), \mathbb{Z}\right) \simeq \operatorname{Hom}(0, \mathbb{Z}) \oplus \operatorname{Ext}\left(\mathbb{Z}_{3}, \mathbb{Z}\right) \simeq 0 \oplus \mathbb{Z}_{3} \simeq \mathbb{Z}_{3}$

Para $q=3$, temos

$$
H^{q}(C ; \mathbb{Z}) \simeq \operatorname{Hom}\left(H_{3}(C), \mathbb{Z}\right) \oplus \operatorname{Ext}\left(H_{2}(C), \mathbb{Z}\right) \simeq \operatorname{Hom}(\mathbb{Z}, \mathbb{Z}) \oplus 0 \simeq \mathbb{Z}
$$

Portanto,

$$
H^{q}(C ; \mathbb{Z})= \begin{cases}\mathbb{Z} & \text { se } q=0,3 \\ 0 & \text { se } q=1 \\ \mathbb{Z}_{3} & \text { se } q=2\end{cases}
$$

\subsubsection{Grupos de cohomologia com coeficientes em $\mathbb{Z}_{2}$}

Agora, calculemos a cohomologia do complexo de cadeias $C$ com coeficientes em $\mathbb{Z}_{2}$. 
Para $q=0$, temos

$$
H^{q}\left(C ; \mathbb{Z}_{2}\right) \simeq \operatorname{Hom}\left(H_{0}(C), \mathbb{Z}_{2}\right) \oplus \operatorname{Ext}\left(H_{-1}(C), \mathbb{Z}_{2}\right) \simeq \operatorname{Hom}\left(\mathbb{Z}, \mathbb{Z}_{2}\right) \oplus 0 \simeq \mathbb{Z}_{2} .
$$

Para $q=1$, temos

$H^{q}\left(C ; \mathbb{Z}_{2}\right) \simeq \operatorname{Hom}\left(H_{1}(C), \mathbb{Z}_{2}\right) \oplus \operatorname{Ext}\left(H_{0}(C), \mathbb{Z}_{2}\right) \simeq \operatorname{Hom}\left(\mathbb{Z}_{3}, \mathbb{Z}_{2}\right) \oplus \operatorname{Ext}\left(\mathbb{Z}, \mathbb{Z}_{2}\right) \simeq 0 \oplus 0 \simeq 0$

Para $q=2$, temos

$H^{q}\left(C ; \mathbb{Z}_{2}\right) \simeq \operatorname{Hom}\left(H_{2}(C), \mathbb{Z}_{2}\right) \oplus \operatorname{Ext}\left(H_{1}(C), \mathbb{Z}_{2}\right) \simeq \operatorname{Hom}\left(0, \mathbb{Z}_{2}\right) \oplus \operatorname{Ext}\left(\mathbb{Z}_{3}, \mathbb{Z}_{2}\right) \simeq 0 \oplus 0 \simeq 0$

Para $q=3$, temos

$$
H^{q}\left(C ; \mathbb{Z}_{2}\right) \simeq \operatorname{Hom}\left(H_{3}(C), \mathbb{Z}_{2}\right) \oplus \operatorname{Ext}\left(H_{2}(C), \mathbb{Z}_{2}\right) \simeq \operatorname{Hom}\left(\mathbb{Z}, \mathbb{Z}_{2}\right) \oplus 0 \simeq \mathbb{Z}_{2} .
$$

Portanto,

$$
H^{q}\left(C ; \mathbb{Z}_{2}\right)= \begin{cases}\mathbb{Z}_{2} & \text { se } q=0,3 \\ 0 & \text { se } q=1,2\end{cases}
$$

\subsubsection{Grupos de cohomologia com coeficientes em $\mathbb{Z}_{3}$}

Calculemos a cohomologia do complexo de cadeias $C$ com coeficientes em $\mathbb{Z}_{3}$.

Para $q=0$, temos

$$
H^{q}\left(C ; \mathbb{Z}_{3}\right) \simeq \operatorname{Hom}\left(H_{0}(C), \mathbb{Z}_{3}\right) \oplus \operatorname{Ext}\left(H_{-1}(C), \mathbb{Z}_{3}\right) \simeq H o m\left(\mathbb{Z}, \mathbb{Z}_{3}\right) \oplus 0 \simeq \mathbb{Z}_{3} .
$$

Para $q=1$, temos

$H^{q}\left(C ; \mathbb{Z}_{3}\right) \simeq \operatorname{Hom}\left(H_{1}(C), \mathbb{Z}_{3}\right) \oplus \operatorname{Ext}\left(H_{0}(C), \mathbb{Z}_{3}\right) \simeq \operatorname{Hom}\left(\mathbb{Z}_{3}, \mathbb{Z}_{3}\right) \oplus \operatorname{Ext}\left(\mathbb{Z}, \mathbb{Z}_{3}\right) \simeq \mathbb{Z}_{3} \oplus 0 \simeq \mathbb{Z}_{3}$ 
Para $q=2$, temos

$H^{q}\left(C ; \mathbb{Z}_{3}\right) \simeq \operatorname{Hom}\left(H_{2}(C), \mathbb{Z}_{3}\right) \oplus \operatorname{Ext}\left(H_{1}(C), \mathbb{Z}_{3}\right) \simeq \operatorname{Hom}\left(0, \mathbb{Z}_{3}\right) \oplus \operatorname{Ext}\left(\mathbb{Z}_{3}, \mathbb{Z}_{3}\right) \simeq 0 \oplus \mathbb{Z}_{3} \simeq \mathbb{Z}_{3}$.

Para $q=3$, temos

$$
H^{q}\left(C ; \mathbb{Z}_{3}\right) \simeq \operatorname{Hom}\left(H_{3}(C), \mathbb{Z}_{3}\right) \oplus \operatorname{Ext}\left(H_{2}(C), \mathbb{Z}_{3}\right) \simeq H o m\left(\mathbb{Z}, \mathbb{Z}_{3}\right) \oplus 0 \simeq \mathbb{Z}_{3} .
$$

Portanto,

$$
H^{q}\left(C ; \mathbb{Z}_{3}\right)=\mathbb{Z}_{3} \text { se } q=0,1,2,3
$$

\subsubsection{Grupos de cohomologia com coeficientes em $\mathbb{Z}_{p}$, com $p$ primo} e $p>3$

Finalmente, calculemos a cohomologia do complexo de cadeias $C$ com coeficientes em $\mathbb{Z}_{p}$, onde $p$ é primo, $p>3$.

Para $q=0$, temos

$$
H^{q}\left(C ; \mathbb{Z}_{p}\right) \simeq \operatorname{Hom}\left(H_{0}(C), \mathbb{Z}_{p}\right) \oplus \operatorname{Ext}\left(H_{-1}(C), \mathbb{Z}_{p}\right) \simeq H o m\left(\mathbb{Z}, \mathbb{Z}_{p}\right) \oplus 0 \simeq \mathbb{Z}_{p}
$$

Para $q=1$, temos

$H^{q}\left(C ; \mathbb{Z}_{p}\right) \simeq \operatorname{Hom}\left(H_{1}(C), \mathbb{Z}_{p}\right) \oplus \operatorname{Ext}\left(H_{0}(C), \mathbb{Z}_{p}\right) \simeq \operatorname{Hom}\left(\mathbb{Z}_{3}, \mathbb{Z}_{p}\right) \oplus \operatorname{Ext}\left(\mathbb{Z}, \mathbb{Z}_{p}\right) \simeq 0$.

Para $q=2$, temos

$H^{q}\left(C ; \mathbb{Z}_{p}\right) \simeq \operatorname{Hom}\left(H_{2}(C), \mathbb{Z}_{p}\right) \oplus \operatorname{Ext}\left(H_{1}(C), \mathbb{Z}_{p}\right) \simeq \operatorname{Hom}\left(0, \mathbb{Z}_{p}\right) \oplus \operatorname{Ext}\left(\mathbb{Z}_{3}, \mathbb{Z}_{p}\right) \simeq 0$

Para $q=3$, temos

$$
H^{q}\left(C ; \mathbb{Z}_{p}\right) \simeq \operatorname{Hom}\left(H_{3}(C), \mathbb{Z}_{p}\right) \oplus \operatorname{Ext}\left(H_{2}(C), \mathbb{Z}_{p}\right) \simeq \operatorname{Hom}\left(\mathbb{Z}, \mathbb{Z}_{p}\right) \oplus 0 \simeq \mathbb{Z}_{p}
$$


Portanto,

$$
H^{q}\left(C ; \mathbb{Z}_{p}\right)= \begin{cases}\mathbb{Z}_{p} & \text { se } q=0,3 \\ 0 & \text { se } q=1,2\end{cases}
$$

Observação 4.3.1 Lembramos que calcular os grupos de cohomologia do complexo de cadeias $C$, nada mais é, do que calcular os grupos de cohomologia da forma espacial esférica tetraedral $\mathscr{P}_{3}$.

\subsection{Anel de cohomologia de $\mathscr{P}_{3}$}

Calculemos agora o anel de cohomologia para a forma espacial esférica tetraedral, de dimensão três, com coeficientes em $\mathbb{Z}, \mathbb{Z}_{2}, \mathbb{Z}_{3}$ e $\mathbb{Z}_{p}$, com $p$ primo e $p>3$, utilizando a interpretação geométrica do produto cup vista na Subseção 4.2.1.

\subsubsection{Anel de cohomologia com coeficientes em $\mathbb{Z}$}

O anel de cohomologia de $\mathscr{P}_{3}=\frac{S^{3}}{\eta\left(P_{24}\right)}$ com coeficientes em $\mathbb{Z}$ é

$$
H^{*}\left(\mathscr{P}_{3} ; \mathbb{Z}\right)=\mathbb{Z}\left[\sigma^{2}, \sigma^{3}\right] /\left(3 \sigma^{2}=0\right) .
$$

De fato,

$$
H^{q}\left(\mathscr{P}_{3} ; \mathbb{Z}\right)= \begin{cases}\mathbb{Z} & \text { se } q=0,3 \\ 0 & \text { se } q=1 \\ \mathbb{Z}_{3} & \text { se } q=2\end{cases}
$$

Portanto, obtemos a seguinte tabela com o produto cup em $H^{*}\left(\mathscr{P}_{3} ; \mathbb{Z}\right)$.

\begin{tabular}{|c|c|c|c|}
\hline$\cup$ & $\sigma^{0}$ & $\sigma^{2}$ & $\sigma^{3}$ \\
\hline$\sigma^{0}$ & $\sigma^{0}$ & $\sigma^{2}$ & $\sigma^{3}$ \\
\hline$\sigma^{2}$ & $\sigma^{2}$ & 0 & 0 \\
\hline$\sigma^{3}$ & $\sigma^{3}$ & 0 & 0 \\
\hline
\end{tabular}




\subsubsection{Anel de cohomologia com coeficientes em $\mathbb{Z}_{2}$}

O anel de cohomologia de $\mathscr{P}_{3}=\frac{S^{3}}{\eta\left(P_{24}\right)}$ com coeficientes em $\mathbb{Z}_{2}$ é

$$
H^{*}\left(\mathscr{P}_{3} ; \mathbb{Z}_{2}\right)=\mathbb{Z}\left[\sigma^{3}\right] /\left(2 . \sigma^{3}=0\right) .
$$

De fato,

$$
H^{q}\left(\mathscr{P}_{3} ; \mathbb{Z}_{2}\right)=\left\{\begin{array}{cc}
\mathbb{Z}_{2} & \text { se } q=0,3 \\
0 & \text { se } q=1,2
\end{array}\right.
$$

Assim, segue a tabela com o produto cup em $H^{*}\left(\mathscr{P}_{3} ; \mathbb{Z}_{2}\right)$.

\begin{tabular}{|c|c|c|}
\hline$\cup$ & $\sigma^{0}$ & $\sigma^{3}$ \\
\hline$\sigma^{0}$ & $\sigma^{0}$ & $\sigma^{3}$ \\
\hline$\sigma^{3}$ & $\sigma^{3}$ & 0 \\
\hline
\end{tabular}

\subsubsection{Anel de cohomologia com coeficientes em $\mathbb{Z}_{3}$}

O anel de cohomologia de $\mathscr{P}_{3}=\frac{S^{3}}{\eta\left(P_{24}\right)}$ com coeficientes em $\mathbb{Z}_{3}$ é

$$
H^{*}\left(\mathscr{P}_{3} ; \mathbb{Z}_{3}\right)=\mathbb{Z}_{3}\left[\sigma^{1}, \sigma^{2}, \sigma^{3}\right] /\left(\sigma^{1} \cup \sigma^{1}=0, \sigma^{1} \cup \sigma^{2}=\sigma^{3}\right) .
$$

De fato,

$$
H^{q}\left(\mathscr{P}_{3} ; \mathbb{Z}_{3}\right)=\left\{\begin{array}{cc}
\mathbb{Z}_{3} & \text { se } q=0 \\
\mathbb{Z}_{3} & \text { se } q=1 \\
\mathbb{Z}_{3} & \text { se } q=2 \\
\mathbb{Z}_{3} & \text { se } q=3
\end{array}\right.
$$

Seja $\sigma^{q}$ o gerador de $H^{q}\left(\mathscr{P}_{3} ; \mathbb{Z}_{3}\right)$, para todo $q$.

Vimos que $H_{0}\left(\mathscr{P}_{3} ; \mathbb{Z}_{3}\right) \simeq \mathbb{Z}_{3}$, com gerador $\left[\widetilde{c}_{0,1}\right], H_{1}\left(\mathscr{P}_{3} ; \mathbb{Z}_{3}\right) \simeq \mathbb{Z}_{3}$, com gerador $\left[\widetilde{c}_{1,4}\right]$, $H_{2}\left(\mathscr{P}_{3} ; \mathbb{Z}_{3}\right) \simeq \mathbb{Z}_{3}$, com gerador $\left[\widetilde{c}_{2,1}+\widetilde{c}_{2,2}-\widetilde{c}_{2,3}-\widetilde{c}_{2,4}\right]$ e $H_{3}\left(\mathscr{P}_{3} ; \mathbb{Z}_{3}\right) \simeq \mathbb{Z}_{3}$, com gerador 
$\left[\widetilde{c}_{3,1}\right]$ (vide Subseção 3.2.3).

Sejam $\left[\widetilde{c}_{0,1}\right]^{*}=\sigma^{3},\left[\widetilde{c}_{1,4}\right]^{*}=\sigma^{2},\left[\widetilde{c}_{2,1}+\widetilde{c}_{2,2}-\widetilde{c}_{2,3}-\widetilde{c}_{2,4}\right]^{*}=\sigma^{1}$ e $\left[\widetilde{c}_{3,1}\right]^{*}=\sigma^{0}$ o dual de Poincaré de $\left[\widetilde{c}_{0,1}\right],\left[\widetilde{c}_{1,4}\right],\left[\widetilde{c}_{2,1}+\widetilde{c}_{2,2}-\widetilde{c}_{2,3}-\widetilde{c}_{2,4}\right]$ e $\left[\widetilde{c}_{3,1}\right]$, respectivamente.

Claramente, $\sigma^{0}$ é o elemento neutro de $H^{*}\left(\mathscr{P}_{3} ; \mathbb{Z}_{3}\right)$.

Considerando positiva a orientação anti-horário, pelo Teorema 4.2.4, temos

$$
\begin{aligned}
\sigma^{1} \cup \sigma^{2} & =\left[\widetilde{c}_{2,1}+\widetilde{c}_{2,2}-\widetilde{c}_{2,3}-\widetilde{c}_{2,4}\right]^{*} \cup\left[\widetilde{c}_{1,4}\right]^{*} \\
& =\left[\left(\widetilde{c}_{2,1}+\widetilde{c}_{2,2}-\widetilde{c}_{2,3}-\widetilde{c}_{2,4}\right) \cap \widetilde{c}_{1,4}\right]^{*} \\
& =\left[\widetilde{c}_{0,1}\right]^{*} \in H^{3}\left(\mathscr{P}_{3} ; \mathbb{Z}_{3}\right) .
\end{aligned}
$$

Logo, $\sigma^{1} \cup \sigma^{2}=\sigma^{3}$.

Analogamente, $\sigma^{2} \cup \sigma^{1}=\sigma^{3}$.

Para ver que a intersecção acima é $\widetilde{c}_{0,1}$, consideramos um representante de $\widetilde{c}_{2,1}+\widetilde{c}_{2,2}-\widetilde{c}_{2,3}-\widetilde{c}_{2,4}$ e um representante de $\widetilde{c}_{1,4}$, como na figura abaixo.

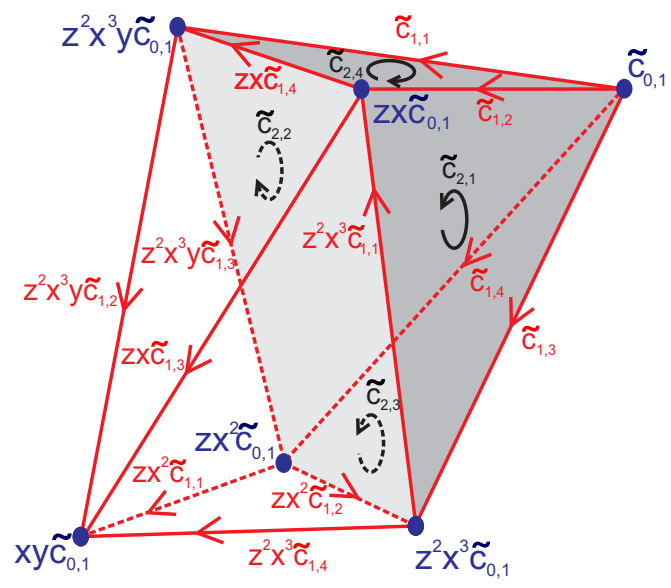

Por outro lado,

$$
\begin{aligned}
\sigma^{1} \cup \sigma^{1} & =\left[\widetilde{c}_{2,1}+\widetilde{c}_{2,2}-\widetilde{c}_{2,3}-\widetilde{c}_{2,4}\right]^{*} \cup\left[\widetilde{c}_{2,1}+\widetilde{c}_{2,2}-\widetilde{c}_{2,3}-\widetilde{c}_{2,4}\right]^{*} \\
& =\left[\left(\widetilde{c}_{2,1}+\widetilde{c}_{2,2}-\widetilde{c}_{2,3}-\widetilde{c}_{2,4}\right) \cap\left(\widetilde{c}_{2,1}+\widetilde{c}_{2,2}-\widetilde{c}_{2,3}-\widetilde{c}_{2,4}\right)\right]^{*} \\
& =\left[\partial_{2}\left(\widetilde{c}_{2,1}+\widetilde{c}_{2,2}-\widetilde{c}_{2,3}-\widetilde{c}_{2,4}\right)\right] \\
& =[0]^{*} \simeq 0 \in H^{2}\left(\mathscr{P}_{3} ; \mathbb{Z}_{3}\right)
\end{aligned}
$$

Para ver que a intersecção acima é 0, consideramos dois representantes de 
$\widetilde{c}_{2,1}+\widetilde{c}_{2,2}-\widetilde{c}_{2,3}-\widetilde{c}_{2,4}$ como nas figuras abaixo.
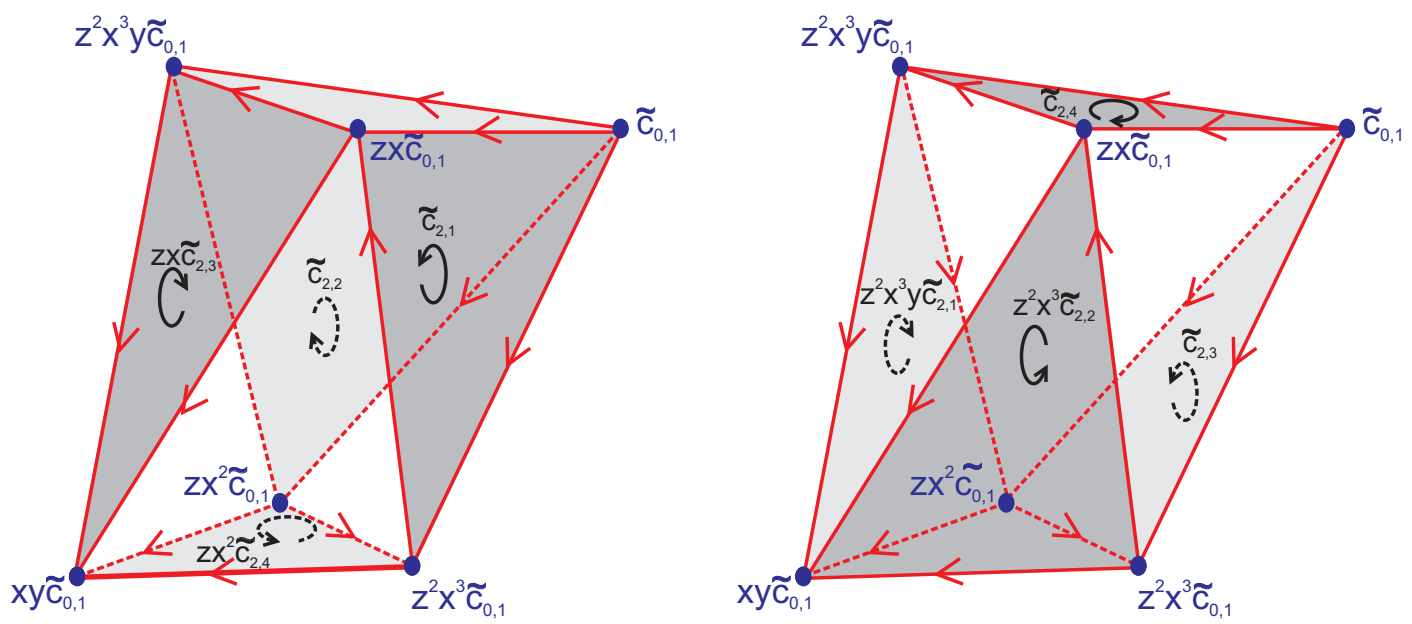

Logo, temos a tabela com o produto cup em $H^{*}\left(\mathscr{P}_{3} ; \mathbb{Z}_{3}\right)$.

\begin{tabular}{|c|c|c|c|c|}
\hline$\cup$ & $\sigma^{0}$ & $\sigma^{1}$ & $\sigma^{2}$ & $\sigma^{3}$ \\
\hline$\sigma^{0}$ & $\sigma^{0}$ & $\sigma^{1}$ & $\sigma^{2}$ & $\sigma^{3}$ \\
\hline$\sigma^{1}$ & $\sigma^{1}$ & 0 & $\sigma^{3}$ & 0 \\
\hline$\sigma^{2}$ & $\sigma^{2}$ & $\sigma_{3}$ & 0 & 0 \\
\hline$\sigma^{3}$ & $\sigma^{3}$ & 0 & 0 & 0 \\
\hline
\end{tabular}

\subsubsection{Anel de cohomologia com coeficientes em $\mathbb{Z}_{p}$, com $p$ primo e} $p>3$

O anel de cohomologia de $\mathscr{P}_{3}=\frac{S^{3}}{\eta\left(P_{24}\right)}$ com coeficientes em $\mathbb{Z}_{p}(p$ primo e $p>3)$ é

$$
H^{*}\left(\mathscr{P}_{3} ; \mathbb{Z}_{p}\right)=\mathbb{Z}\left[\sigma^{3}\right] /\left(p \cdot \sigma^{3}=0\right)
$$

De fato,

$$
H^{q}\left(\mathscr{P}_{3} ; \mathbb{Z}_{p}\right)=\left\{\begin{array}{cc}
\mathbb{Z}_{p} & \text { se } q=0,3 \\
0 & \text { se } q=1,2
\end{array}\right.
$$

Dessa forma, segue a tabela com o produto cup em $H^{*}\left(\mathscr{P}_{3} ; \mathbb{Z}_{p}\right)$. 


\begin{tabular}{|c|c|c|}
\hline$\cup$ & $\sigma^{0}$ & $\sigma^{3}$ \\
\hline$\sigma^{0}$ & $\sigma^{0}$ & $\sigma^{3}$ \\
\hline$\sigma^{3}$ & $\sigma^{3}$ & 0 \\
\hline
\end{tabular}

\subsection{Homotopia contrátil}

Definição 4.5.1 Sejam $C=\left\{C_{n}, \partial_{n}\right\}_{n \geq 0}\left(\partial_{0}=0\right)$ e $C^{\prime}=\left\{C_{n}^{\prime}, \partial_{n}^{\prime}\right\}_{n \geq 0}\left(\partial_{0}^{\prime}=0\right)$ complexos de cadeias de R-módulos. Dizemos que uma aplicação de cadeias $\varphi: C \rightarrow C^{\prime}$ é uma sequência de $R$-aplicações $\varphi_{n}: C_{n} \rightarrow C_{n}^{\prime}$ tais que o diagrama

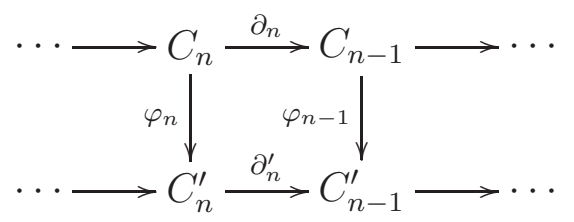

comuta para cada $n$.

Definição 4.5.2 Sejam $\varphi, \psi: C \rightarrow C^{\prime}$ duas aplicações de cadeias de $C=\left\{C_{n}, \partial_{n}\right\}$ em $C^{\prime}=\left\{C_{n}^{\prime}, \partial_{n}^{\prime}\right\}$. Suponha que s é uma sequência de homomorfismos de $\mathbb{Z}$-módulos (grupo abeliano) $s_{n}: C_{n} \rightarrow C_{n+1}^{\prime}$ satisfazendo $\partial_{n+1}^{\prime} s_{n}+s_{n-1} \partial_{n}=\psi_{n}-\varphi_{n}: C_{n} \rightarrow C_{n}^{\prime}$, para todo $n$. Então, dizemos que s é uma homotopia de cadeias de $\varphi$ em $\psi$, e a denotamos por $s: \varphi \simeq \psi$.

Definição 4.5.3 Uma homotopia contrátil para o complexo de cadeias $C$ é uma homotopia de cadeias $s: 0 \simeq 1_{C}: C \rightarrow C$, onde $0: C \rightarrow C$ denota a aplicação nula e $1_{C}: C \rightarrow C$ denota a aplicação identidade.

Proposição 4.5.4 ([21], Corolário 5.4) Se o complexo de cadeias $C$ admite uma homotopia contrátil s então $C$ é acíclico (isto é, $H_{n}(C)=0$ para todo $n$, ou seja, $C$ é uma sequência exata).

Observação 4.5.5 Dizemos que s é uma homotopia contrátil para a resolução $C \stackrel{\varepsilon}{\rightarrow} \mathbb{Z}$ de $\mathbb{Z}$ sobre $R$, se ela é uma homotopia contrátil para o complexo de cadeias $C$, com $C_{-1}=\mathbb{Z}$ $e C_{n}=0, n<-1$. Em particular, $\varepsilon s_{-1}=1_{\mathbb{Z}}, \partial_{1} s_{0}+s_{-1} \varepsilon=1_{C_{0}}$ e $\partial_{n+1} s_{n}+s_{n-1} \partial_{n}=1_{C_{n}}$, para todo $n>0$. 
Vamos então, definir uma homotopia contrátil $s=\left\{s_{n}\right\}, n \geq-1$ para a resolução 4-periódica encontrada no Corolário 2.3.5. Como vimos anteriormente, devemos definir $\mathbb{Z}$-homomorfismos $s_{n}: C_{n} \rightarrow C_{n+1}$ que satisfazem o seguinte diagrama comutativo:

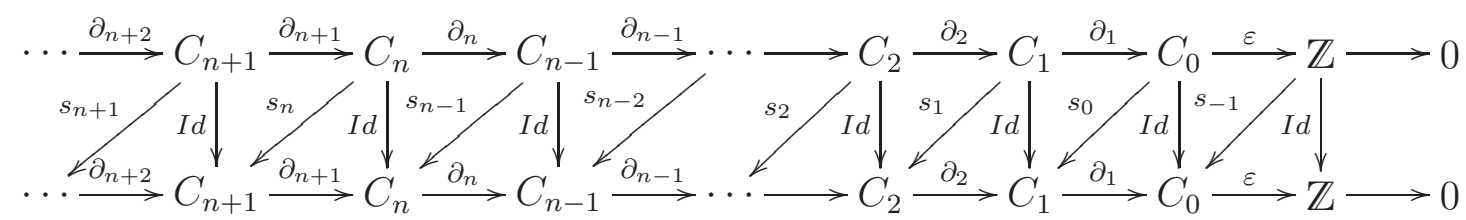

Em outras palavras, $\partial_{1} s_{0}+s_{-1} \varepsilon=1_{C_{0}}$ e $\partial_{n+1} s_{n}+s_{n-1} \partial_{n}=1_{C_{n}}$, para todo $n>0$.

Teorema 4.5.6 Uma homotopia contrátil para o complexo de cadeias

$$
\begin{gathered}
C: \cdots \longrightarrow \mathbb{Z} P_{24}\left[\widetilde{c}_{5,1}, \widetilde{c}_{5,2}, \widetilde{c}_{5,3}, \widetilde{c}_{5,4}\right] \stackrel{\partial_{5}}{\longrightarrow} \mathbb{Z} P_{24}\left[\widetilde{c}_{4,1}\right] \stackrel{\partial_{4}}{\longrightarrow} \mathbb{Z} P_{24}\left[\widetilde{c}_{3,1}\right] \stackrel{\partial_{3}}{\longrightarrow} \\
\stackrel{\partial_{3}}{\longrightarrow} \mathbb{Z} P_{24}\left[\widetilde{c}_{2,1}, \widetilde{c}_{2,2}, \widetilde{c}_{2,3}, \widetilde{c}_{2,4}\right] \stackrel{\partial_{2}}{\longrightarrow} \mathbb{Z} P_{24}\left[\widetilde{c}_{1,1}, \widetilde{c}_{1,2}, \widetilde{c}_{1,3}, \widetilde{c}_{1,4}\right] \stackrel{\partial_{1}}{\longrightarrow} \mathbb{Z} P_{24}\left[\widetilde{c}_{0,1}\right] \stackrel{\varepsilon}{\longrightarrow} \mathbb{Z} \longrightarrow 0
\end{gathered}
$$

é dada como a seguir:

- Definição da $s_{-1}$ :

$s_{-1}(1)=\widetilde{c}_{0,1}$

- $\underline{\text { Definição da } s_{0}}$ :

1) $s_{0}\left(\widetilde{c}_{0,1}\right)=0$

2) $s_{0}\left(x \widetilde{c}_{0,1}\right)=\left(1+z x^{2}\right) \widetilde{c}_{1,4}+z^{2} \widetilde{c}_{1,2}$

3) $s_{0}\left(x^{2} \widetilde{c}_{0,1}\right)=\left(1+z^{2} x^{3} y+\left(z^{2} x^{3} y\right)^{2}\right) \widetilde{c}_{1,1}$

4) $s_{0}\left(x^{3} \widetilde{c}_{0,1}\right)=\left(1+z x^{2}+\left(z x^{2}\right)^{2}+\left(z x^{2}\right)^{3}\right) \widetilde{c}_{1,4}+z \widetilde{c}_{1,3}$

5) $s_{0}\left(y \widetilde{c}_{0,1}\right)=\left(1+z^{2} x^{3}+\left(z^{2} x^{3}\right)^{2}+\left(z^{2} x^{3}\right)^{3}+\left(z^{2} x^{3}\right)^{4}\right) \widetilde{c}_{1,3}+z x y \widetilde{c}_{1,1}$

6) $s_{0}\left(x y \widetilde{c}_{0,1}\right)=\widetilde{c}_{1,4}+z x^{2} \widetilde{c}_{1,1}$

7) $s_{0}\left(x^{2} y \widetilde{c}_{0,1}\right)=\left(1+z^{2} x^{3}\right) \widetilde{c}_{1,3}+z x^{3} y \widetilde{c}_{1,1}$

8) $s_{0}\left(x^{3} y \widetilde{c}_{0,1}\right)=\left(1+z x^{2}+\left(z x^{2}\right)^{2}+\left(z x^{2}\right)^{3}\right) \widetilde{c}_{1,4}+z \widetilde{c}_{1,1}$ 
9) $s_{0}\left(z \widetilde{c}_{0,1}\right)=\left(1+z x^{2}+\left(z x^{2}\right)^{2}+\left(z x^{2}\right)^{3}\right) \widetilde{c}_{1,4}$

10) $s_{0}\left(z x \widetilde{c}_{0,1}\right)=\widetilde{c}_{1,2}$

11) $s_{0}\left(z x^{2} \widetilde{c}_{0,1}\right)=\widetilde{c}_{1,4}$

12) $s_{0}\left(z x^{3} \widetilde{c_{0}}\right)=\left(1+z x+(z x)^{2}+(z x)^{3}\right) \widetilde{c}_{1,2}$

13) $s_{0}\left(z y \widetilde{c}_{0,1}\right)=\left(1+z^{2} x^{3} y+\left(z^{2} x^{3} y\right)^{2}+\left(z^{2} x^{3} y\right)^{3}+\left(z^{2} x^{3} y\right)^{4}\right) \widetilde{c}_{1,1}$

14) $s_{0}\left(z x y \widetilde{c}_{0,1}\right)=\left(1+z^{2} x^{3}+\left(z^{2} x^{3}\right)^{2}+\left(z^{2} x^{3}\right)^{3}+\left(z^{2} x^{3}\right)^{4}\right) \widetilde{c}_{1,3}$

15) $s_{0}\left(z x^{2} y \widetilde{c}_{0,1}\right)=\left(1+z^{2} x^{3} y\right) \widetilde{c}_{1,1}$

16) $s_{0}\left(z x^{3} y \widetilde{c}_{0,1}\right)=\left(1+z^{2} x^{3}\right) \widetilde{c}_{1,3}$

17) $s_{0}\left(z^{2} \widetilde{c}_{0,1}\right)=\left(1+z x^{2}\right) \widetilde{c}_{1,4}$

18) $s_{0}\left(z^{2} x \widetilde{c}_{0,1}\right)=\left(1+z^{2} x^{3}+\left(z^{2} x^{3}\right)^{2}+\left(z^{2} x^{3}\right)^{3}\right) \widetilde{c}_{1,3}$

19) $s_{0}\left(z^{2} x^{2} \widetilde{c}_{0,1}\right)=\left(1+z x^{2}+\left(z x^{2}\right)^{2}+\left(z x^{2}\right)^{3}+\left(z x^{2}\right)^{4}\right) \widetilde{c}_{1,4}$

20) $s_{0}\left(z^{2} x^{3} \widetilde{c}_{0,1}\right)=\widetilde{c}_{1,3}$

21) $s_{0}\left(z^{2} y \widetilde{c}_{0,1}\right)=(1+z x) \widetilde{c}_{1,2}$

22) $s_{0}\left(z^{2} x y \widetilde{c}_{0,1}\right)=\left(1+z^{2} x^{3} y+\left(z^{2} x^{3} y\right)^{2}+\left(z^{2} x^{3} y\right)^{3}\right) \widetilde{c}_{1,1}$

23) $s_{0}\left(z^{2} x^{2} y \widetilde{c}_{0,1}\right)=\left(1+z x+(z x)^{2}+(z x)^{3}+(z x)^{4}\right) \widetilde{c}_{1,2}$

24) $s_{0}\left(z^{2} x^{3} y \widetilde{c}_{0,1}\right)=\widetilde{c}_{1,1}$

- Definição da $s_{1}$ :

1) $s_{1}\left(\widetilde{c}_{1,1}\right)=0$

2) $s_{1}\left(x \widetilde{c}_{1,1}\right)=-z x^{2} \widetilde{c}_{2,1}+\left(-1-z x^{2}\right) \widetilde{c}_{2,3}$

3) $s_{1}\left(x^{2} \widetilde{c}_{1,1}\right)=0$

4) $s_{1}\left(x^{3} \widetilde{c}_{1,1}\right)=\left(1-x y-z x+z x^{2}-z x^{3}+z y-z x y+z x^{2} y-z x^{3} y+z^{2}+z^{2} x+\right.$ $\left.+z^{2} x^{2}+z^{2} x^{3}-z^{2} y-z^{2} x y-z^{2} x^{2} y-2 z^{2} x^{3} y\right) \widetilde{c}_{2,1}+\left(2-x+x^{2}-x^{3}-y+x y-\right.$ 
$\left.-x^{2} y+x^{3} y+z-z x+2 z x^{2}-z x^{3}+z y-z x y+z x^{2} y-z x^{3} y+z^{2}-z^{2} x^{3}\right) \widetilde{c}_{2,2}+(1-$ $\left.-x-x^{3}-y+x y-x^{2} y+x^{3} y+z-2 z x+z x^{2}-z x^{3}+z y-z x y+z x^{2} y-z x^{3} y-z^{2} x^{3}\right) \widetilde{c}_{2,3}+\widetilde{c}_{2,4}$

5) $s_{1}\left(y \widetilde{c}_{1,1}\right)=\left(-1-z x^{2} y-z^{2} x+z^{2} y+z^{2} x^{3} y\right) \widetilde{c}_{2,1}+\left(-1-x y-z x^{2}-z x^{2} y+\right.$ $\left.+z^{2} x^{3}\right) \widetilde{c}_{2,2}+\left(-x y+z x-z x^{2} y+z x^{3} y+z^{2} x^{3}\right) \widetilde{c}_{2,3}+(-1-x y) \widetilde{c}_{2,4}$

6) $s_{1}\left(x y \widetilde{c}_{1,1}\right)=\left(-z x-z^{2} x^{3} y\right) \widetilde{c}_{2,1}+\widetilde{c}_{2,2}-z x \widetilde{c}_{2,3}+\widetilde{c}_{2,4}$

7) $s_{1}\left(x^{2} y \widetilde{c}_{1,1}\right)=\left(-1+y+x y+z x^{3}-z y+z x^{3} y-z^{2}-z^{2} x^{3}+z^{2} x y+z^{2} x^{3} y\right) \widetilde{c}_{2,1}+$ $\left(-1+x-x^{2}+y-x^{3} y-z-z x^{2}+z x^{3}-z^{2}+z^{2} x^{3}-z^{2} x^{2} y\right) \widetilde{c}_{2,2}+\left(x+y-x^{3} y+z x-\right.$ $\left.-z x^{2}+z x^{3}-z y+z^{2} x^{3}-z^{2} x^{2} y\right) \widetilde{c}_{2,3}+(-1+x+y) \widetilde{c}_{2,4}$

8) $s_{1}\left(x^{3} y \widetilde{c}_{1,1}\right)=\left(1-2 x y-z x+z x^{2}-z x^{3}-z x^{3} y+z^{2}-z^{2} x y-2 z^{2} x^{3} y\right) \widetilde{c}_{2,1}+(2-$ $\left.-x+x^{2}+2 z x^{2}-z x^{3}+2 z^{2}-z^{2} x^{3}\right) \widetilde{c}_{2,2}+\left(1-x-2 z x+z x^{2}-z x^{3}+z x^{3} y+z^{2}-\right.$ $\left.-z^{2} x^{3}\right) \widetilde{c}_{2,3}+(2-x y) \widetilde{c}_{2,4}$

9) $s_{1}\left(z \widetilde{c}_{1,1}\right)=0$

10) $s_{1}\left(z x \widetilde{c}_{1,1}\right)=\left(y+x y+z x+z x^{3}-z y+z x^{3} y-z^{2}-z^{2} x^{2}-z^{2} x^{3}+z^{2} x y+z^{2} x^{3} y\right) \widetilde{c}_{2,1}+$ $+\left(-1+x-x^{2}+y+x^{2} y-x^{3} y-z-z x^{2}+z x^{3}+z x^{3} y-z^{2}-z^{2} x^{2} y\right) \widetilde{c}_{2,2}+(x+y+$ $\left.+x^{2} y-x^{3} y-z+z x-z x^{2}+z x^{3}-z y-z^{2} x^{2} y\right) \widetilde{c}_{2,3}+(-1+x+y+x y) \widetilde{c}_{2,4}$

11) $s_{1}\left(z x^{2} \widetilde{c}_{1,1}\right)=0$

12) $s_{1}\left(z x^{3} \widetilde{c}_{1,1}\right)=\left(x y+z x-z^{2}+z^{2} x^{3} y\right) \widetilde{c}_{2,1}+\left(-1-z x^{2}-z^{2}\right) \widetilde{c}_{2,2}+\left(z x-z x^{3} y-\right.$ $\left.-z^{2}\right) \widetilde{c}_{2,3}+(-1+x y) \widetilde{c}_{2,4}$

13) $s_{1}\left(z y \widetilde{c}_{1,1}\right)=\left(1-2 x-x^{3}+y+x^{2} y+z x+z x^{3}-z y-z x^{3} y-z^{2}-z^{2} x^{2}-z^{2} y+\right.$ $\left.+z^{2} x y\right) \widetilde{c}_{2,1}+\left(-x+y+x^{2} y-x^{3} y-z+z x+z x^{3}-z y-z^{2} x^{3}-z^{2} y+z^{2} x y-z^{2} x^{2} y\right) \widetilde{c}_{2,2}+(-x+$ $\left.+y+x^{2} y-x^{3} y-z-z x^{2}+z x^{3}-z x^{3} y-z^{2} x^{3}-z^{2} y+z^{2} x y-z^{2} x^{2} y\right) \widetilde{c}_{2,3}+(1-x+y+x y) \widetilde{c}_{2,4}$

14) $s_{1}\left(z x y \widetilde{c}_{1,1}\right)=0$

15) $s_{1}\left(z x^{2} y \widetilde{c}_{1,1}\right)=0$

16) $s_{1}\left(z x^{3} y \widetilde{c}_{1,1}\right)=0$

17) $s_{1}\left(z^{2} \widetilde{c}_{1,1}\right)=\left(1-x y-z^{2} x^{3} y\right) \widetilde{c}_{2,1}+\left(1+z x^{2}-z^{2} x^{3}\right) \widetilde{c}_{2,2}+\left(-z x-z^{2} x^{3}\right) \widetilde{c}_{2,3}+\widetilde{c}_{2,4}$ 
18) $s_{1}\left(z^{2} x \widetilde{c}_{1,1}\right)=\left(-1-x^{2}-z x\right) \widetilde{c}_{2,1}+z^{2} x^{3} \widetilde{c}_{2,2}+\left(z x^{3} y+z^{2} x^{3}\right) \widetilde{c}_{2,3}-x y \widetilde{c}_{2,4}$

19) $s_{1}\left(z^{2} x^{2} \widetilde{c}_{1,1}\right)=\left(1-x y-z x+z x^{2}-z x^{3}+z y+z x^{2} y-z x^{3} y+z^{2}+z^{2} x+z^{2} x^{3}-\right.$ $\left.-z^{2} y-z^{2} x y-z^{2} x^{2} y-2 z^{2} x^{3} y\right) \widetilde{c}_{2,1}+\left(2-x+x^{2}-y+x y-x^{2} y+x^{3} y+z+2 z x^{2}-\right.$ $\left.-z x^{3}+z y-z x y+z x^{2} y-z x^{3} y+z^{2}-z^{2} x^{2}-z^{2} x^{3}\right) \widetilde{c}_{2,2}+\left(1-x-y+x y-x^{2} y+x^{3} y-\right.$ $\left.-2 z x+z x^{2}-z x^{3}+z y-z x y+z x^{2} y-z x^{3} y-z^{2} x^{2}-z^{2} x^{3}\right) \widetilde{c}_{2,3}+2 \widetilde{c}_{2,4}$

20) $s_{1}\left(z^{2} x^{3} \widetilde{c}_{1,1}\right)=-\widetilde{c}_{2,1}$

21) $s_{1}\left(z^{2} y \widetilde{c}_{1,1}\right)=\left(x+x y-x^{2} y+z x+z x^{3} y-z^{2} x^{3}+z^{2} x^{3} y\right) \widetilde{c}_{2,1}+\left(-1+x-x^{2}-\right.$ $\left.-z x^{2}+z x^{3} y-z^{2}-z^{2} x y\right) \widetilde{c}_{2,2}+\left(x+z x-z y-z^{2} x y\right) \widetilde{c}_{2,3}+(-1+x+x y) \widetilde{c}_{2,4}$

22) $s_{1}\left(z^{2} x y \widetilde{c}_{1,1}\right)=0$

23) $s_{1}\left(z^{2} x^{2} y \widetilde{c}_{1,1}\right)=\left(-y+x y+z x+z^{2} x^{3} y\right) \widetilde{c}_{2,1}+\left(-1-y-z x^{2}-z^{2}\right) \widetilde{c}_{2,2}+(-y+$ $\left.+z x-z x^{3} y-z^{2}\right) \widetilde{c}_{2,3}+(-1-y+x y) \widetilde{c}_{2,4}$

24) $s_{1}\left(z^{2} x^{3} y \widetilde{c}_{1,1}\right)=0$

25) $s_{1}\left(\widetilde{c}_{1,2}\right)=0$

26) $s_{1}\left(x \widetilde{c}_{1,2}\right)=\left(-1+2 x+x^{3}-y-x^{2} y-z x-z x^{2}-z x^{3}+z y+z x^{3} y+z^{2}+z^{2} x^{2}+\right.$ $\left.+z^{2} y-z^{2} x y\right) \widetilde{c}_{2,1}+\left(x-y-x^{2} y+x^{3} y+z-z x-z x^{3}+z^{2} x^{3}+z^{2} y-z^{2} x y+z^{2} x^{2} y\right) \widetilde{c}_{2,2}+$ $+\left(-1+x-y-x^{2} y+x^{3} y+z-z x^{3}+z x^{3} y+z^{2} x^{3}+z^{2} y-z^{2} x y+z^{2} x^{2} y\right) \widetilde{c}_{2,3}+(-1-y-x y) \widetilde{c}_{2,4}$

27) $s_{1}\left(x^{2} \widetilde{c}_{1,2}\right)=\left(-x-x^{3}+y+x^{2} y+z x^{3}-z y-z^{2}-z^{2} x^{2}-z^{2} y+z^{2} x y\right) \widetilde{c}_{2,1}+(y+$ $\left.+x^{2} y-x^{3} y-z+z x+z x^{3}-z^{2} y+z^{2} x y-z^{2} x^{2} y\right) \widetilde{c}_{2,2}+\left(y+x^{2} y-x^{3} y-z-z x^{2}+z x^{3}-\right.$ $\left.-z^{2} y+z^{2} x y-z^{2} x^{2} y\right) \widetilde{c}_{2,3}+(1+y) \widetilde{c}_{2,4}$

28) $s_{1}\left(x^{3} \widetilde{c}_{1,2}\right)=\left(x^{3}-y-x y-z x-z x^{3}+z y-z x^{3} y+z^{2}+z^{2} x^{2}+z^{2} x^{3}-z^{2} x y-\right.$ $\left.-z^{2} x^{3} y\right) \widetilde{c}_{2,1}+\left(1-x+x^{2}-y-x^{2} y+x^{3} y+z-z x+z x^{2}-z x^{3}-z x^{3} y+z^{2}+z^{2} x^{2} y\right) \widetilde{c}_{2,2}+$ $+\left(-x-y-x^{2} y+x^{3} y+z-z x+z x^{2}-z x^{3}+z y+z^{2} x^{2} y\right) \widetilde{c}_{2,3}+(-x-y-x y) \widetilde{c}_{2,4}$

29) $s_{1}\left(y \widetilde{c}_{1,2}\right)=\left(-1-z x^{2} y-z^{2} x+z^{2} y+z^{2} x^{3} y\right) \widetilde{c}_{2,1}+\left(-1-x y-z x^{2}-z x^{2} y+\right.$ $\left.+z^{2} x^{3}\right) \widetilde{c}_{2,2}+\left(-x y+z x-z x^{2} y+z x^{3} y+z^{2} x^{3}\right) \widetilde{c}_{2,3}+(-1-y-x y) \widetilde{c}_{2,4}$

30) $s_{1}\left(x y \widetilde{c}_{1,2}\right)=\left(1-z^{2} x^{3} y\right) \widetilde{c}_{2,1}+\left(1-z^{2} x^{3}\right) \widetilde{c}_{2,2}+\left(-z x-z^{2} x^{3}\right) \widetilde{c}_{2,3}+\widetilde{c}_{2,4}$

31) $s_{1}\left(x^{2} y \widetilde{c}_{1,2}\right)=\left(-1+x+x y+z x^{3} y-z^{2} x^{3}+z^{2} x^{3} y\right) \widetilde{c}_{2,1}+\left(-1+x-x^{2}-z x^{2}-\right.$ 
$\left.-z^{2}+z^{2} x^{3}-z^{2} x y\right) \widetilde{c}_{2,2}+\left(x+z x-z y+z^{2} x^{3}-z^{2} x y\right) \widetilde{c}_{2,3}+(-1+x) \widetilde{c}_{2,4}$

32) $s_{1}\left(x^{3} y \widetilde{c}_{1,2}\right)=\left(2+x^{2}-2 x y+z x^{2}-z x^{3} y+z^{2}-z^{2} x y-2 z^{2} x^{3} y\right) \widetilde{c}_{2,1}+\left(2-x+x^{2}+\right.$ $\left.+2 z x^{2}-z x^{3}+2 z^{2}-z^{2} x-2 z^{2} x^{3}\right) \widetilde{c}_{2,2}+\left(1-x-2 z x+z x^{2}-z x^{3}+z^{2}-z^{2} x-2 z^{2} x^{3}\right) \widetilde{c}_{2,3}+2 \widetilde{c}_{2,4}$

33) $s_{1}\left(z \widetilde{c}_{1,2}\right)=\left(1-x y-z^{2} x^{3} y\right) \widetilde{c}_{2,1}+\left(1+z x^{2}+z^{2}-z^{2} x^{3}\right) \widetilde{c}_{2,2}+\left(-x^{2}-z x-z^{2} x^{3}\right) \widetilde{c}_{2,3}+\widetilde{c}_{2,4}$

34) $s_{1}\left(z x \widetilde{c}_{1,2}\right)=0$

35) $s_{1}\left(z x^{2} \widetilde{c}_{1,2}\right)=-\widetilde{c}_{2,3}$

36) $s_{1}\left(z x^{3} \widetilde{c}_{1,2}\right)=0$

37) $s_{1}\left(z y \widetilde{c}_{1,2}\right)=\left(-2 x-x^{3}+2 y+x y+x^{2} y+z x+2 z x^{3}-z y-2 z^{2}-z^{2} x^{2}-z^{2} y+\right.$ $\left.+2 z^{2} x y+z^{2} x^{3} y\right) \widetilde{c}_{2,1}+\left(-1-x^{2}+2 y+x^{2} y-2 x^{3} y-2 z+z x-z x^{2}+2 z x^{3}-z^{2}-z^{2} y+\right.$ $\left.+z^{2} x y-2 z^{2} x^{2} y\right) \widetilde{c}_{2,2}+\left(2 y+x^{2} y-2 x^{3} y-z+z x-2 z x^{2}+2 z x^{3}-z x^{3} y-z^{2} y+z^{2} x y-\right.$ $\left.-2 z^{2} x^{2} y\right) \widetilde{c}_{2,3}+(2 y+x y) \widetilde{c}_{2,4}$

38) $s_{1}\left(z x y \widetilde{c}_{1,2}\right)=\left(-1-y-z x^{2}+z x y-z x^{2} y-z^{2} x+z^{2} y+z^{2} x^{2} y+z^{2} x^{3} y\right) \widetilde{c}_{2,1}+$ $+\left(-1-x y-z x^{2}-z y+z x y-z x^{2} y+z^{2} x^{3}+z^{2} x^{2} y\right) \widetilde{c}_{2,2}+(-1-x y+z x+z x y-$ $\left.-z x^{2} y+z x^{3} y+z^{2} x^{3}+z^{2} x^{2} y\right) \widetilde{c}_{2,3}+(-1-x-y-x y) \widetilde{c}_{2,4}$

39) $s_{1}\left(z x^{2} y \widetilde{c}_{1,2}\right)=\left(-x-x^{3}+y+x^{2} y+z x+z x^{3}-z y-z^{2}-z^{2} x^{2}+z^{2} x y+z^{2} x^{3} y\right) \widetilde{c}_{2,1}+$ $+\left(-1+y-x y+x^{2} y-x^{3} y-z+z x-z x^{2}+z x^{3}-z^{2} y+z^{2} x y-z^{2} x^{2} y\right) \widetilde{c}_{2,2}+(y-x y+$ $\left.+x^{2} y-x^{3} y-z+z x-z x^{2}+z x^{3}-z^{2} y+z^{2} x y-z^{2} x^{2} y\right) \widetilde{c}_{2,3}+y \widetilde{c}_{2,4}$

40) $s_{1}\left(z x^{3} y \widetilde{c}_{1,2}\right)=\left(-1+x+x^{3}-y-x^{2} y-z x-z x^{3}+z y+z x^{3} y+z^{2}+z^{2} x^{2}+\right.$ $\left.+z^{2} y-z^{2} x y\right) \widetilde{c}_{2,1}+\left(-y-x^{2} y+x^{3} y+z-z x-z x^{3}+z^{2} x^{3}+z^{2} y-z^{2} x y+z^{2} x^{2} y\right) \widetilde{c}_{2,2}+$ $+\left(-y-x^{2} y+x^{3} y+z+z x^{2}-z x^{3}+z x^{3} y+z^{2} x^{3}+z^{2} y-z^{2} x y+z^{2} x^{2} y\right) \widetilde{c}_{2,3}+(-1-y-x y) \widetilde{c}_{2,4}$

41) $s_{1}\left(z^{2} \widetilde{c}_{1,2}\right)=0$

42) $s_{1}\left(z^{2} x \widetilde{c}_{1,2}\right)=z^{2} x \widetilde{c}_{2,1}$

43) $s_{1}\left(z^{2} x^{2} \widetilde{c}_{1,2}\right)=-z \widetilde{c}_{2,3}$

44) $s_{1}\left(z^{2} x^{3} \widetilde{c}_{1,2}\right)=z^{2} x^{3} \widetilde{c}_{2,1}$

45) $s_{1}\left(z^{2} y \widetilde{c}_{1,2}\right)=\left(x+x^{3}-y-x^{2} y-z x^{3}+z y+z^{2}+z^{2} x^{2}+z^{2} y-z^{2} x y\right) \widetilde{c}_{2,1}+$ 
$+\left(-y-x^{2} y+x^{3} y+z-z x-z x^{3}+z^{2} y-z^{2} x y+z^{2} x^{2} y\right) \widetilde{c}_{2,2}+\left(-y-x^{2} y+x^{3} y+z+\right.$ $\left.+z x^{2}-z x^{3}+z^{2} y-z^{2} x y+z^{2} x^{2} y\right) \widetilde{c}_{2,3}+(-1-y) \widetilde{c}_{2,4}$

46) $s_{1}\left(z^{2} x y \widetilde{c}_{1,2}\right)=\left(-x-x^{3}+y+x y+x^{2} y+z x+z x^{3}-z y-z^{2}-z^{2} x^{2}-z^{2} y+\right.$ $\left.+2 z^{2} x y+z^{2} x^{3} y\right) \widetilde{c}_{2,1}+\left(-1-x^{2}+y+x^{2} y-x^{3} y-z+z x-z x^{2}+z x^{3}-z^{2}-z^{2} y+z^{2} x y-\right.$ $\left.-z^{2} x^{2} y\right) \widetilde{c}_{2,2}+\left(y+x^{2} y-x^{3} y-z+z x-z x^{2}+z x^{3}-z x^{3} y-z^{2} y+z^{2} x y-z^{2} x^{2} y\right) \widetilde{c}_{2,3}+(y+x y) \widetilde{c}_{2,4}$

47) $s_{1}\left(z^{2} x^{2} y \widetilde{c}_{1,2}\right)=\left(-y+x y+z x-z x^{2}+z^{2} x^{2} y+z^{2} x^{3} y\right) \widetilde{c}_{2,1}+\left(-1-y-z x^{2}-\right.$ $\left.-z y-z^{2}+z^{2} x^{2} y\right) \widetilde{c}_{2,2}+\left(-1-y+z x-z x^{3} y-z^{2}+z^{2} x^{2} y\right) \widetilde{c}_{2,3}+(-1-x-y+x y) \widetilde{c}_{2,4}$

48) $s_{1}\left(z^{2} x^{3} y \widetilde{c}_{1,2}\right)=z^{2} x^{3} y \widetilde{c}_{2,1}-\widetilde{c}_{2,2}$

49) $s_{1}\left(\widetilde{c}_{1,3}\right)=0$

50) $s_{1}\left(x \widetilde{c}_{1,3}\right)=\left(-1+x+x^{3}-y-x^{2} y-z x-z x^{2}-z x^{3}+z y+z x^{3} y+z^{2}+z^{2} x^{2}+\right.$ $\left.+z^{2} y-z^{2} x y\right) \widetilde{c}_{2,1}+\left(x-y-x^{2} y+x^{3} y+z-z x-z x^{3}+z^{2} x^{3}+z^{2} y-z^{2} x y+z^{2} x^{2} y\right) \widetilde{c}_{2,2}+$ $+\left(-1+x-y-x^{2} y+x^{3} y+z-z x^{3}+z x^{3} y+z^{2} x^{3}+z^{2} y-z^{2} x y+z^{2} x^{2} y\right) \widetilde{c}_{2,3}+(-1-y-x y) \widetilde{c}_{2,4}$

51) $s_{1}\left(x^{2} \widetilde{c}_{1,3}\right)=\left(1-x-x^{3}+y+x^{2} y+z x+z x^{3}-z y-z^{2}-z^{2} x^{2}-z^{2} y+z^{2} x y\right) \widetilde{c}_{2,1}+$ $+\left(y+x^{2} y-x^{3} y-z+z x+z x^{3}-z^{2} x^{3}-z^{2} y+z^{2} x y-z^{2} x^{2} y\right) \widetilde{c}_{2,2}+\left(y+x^{2} y-x^{3} y-z-\right.$ $\left.-z x^{2}+z x^{3}-z x^{3} y-z^{2} x^{3}-z^{2} y+z^{2} x y-z^{2} x^{2} y\right) \widetilde{c}_{2,3}+(1+y+x y) \widetilde{c}_{2,4}$

52) $s_{1}\left(x^{3} \widetilde{c}_{1,3}\right)=\left(-y-x y-z x-z x^{3}+z y-z x^{3} y+z^{2}+z^{2} x^{2}+z^{2} x^{3}-z^{2} x y-\right.$ $\left.-z^{2} x^{3} y\right) \widetilde{c}_{2,1}+\left(1-x+x^{2}-y-x^{2} y+x^{3} y+z-z x+z x^{2}-z x^{3}-z x^{3} y+z^{2}+z^{2} x^{2} y\right) \widetilde{c}_{2,2}+$ $+\left(-x-y-x^{2} y+x^{3} y+z-z x+z x^{2}-z x^{3}+z y+z^{2} x^{2} y\right) \widetilde{c}_{2,3}+(-x-y-x y) \widetilde{c}_{2,4}$

53) $s_{1}\left(y \widetilde{c}_{1,3}\right)=\left(-1-x y-z x-z x^{2} y-z^{2} x+z^{2} y\right) \widetilde{c}_{2,1}+\left(y-x y-z x^{2} y+z^{2}+z^{2} x^{3}\right) \widetilde{c}_{2,2}+$ $\left(y-x y-z x^{2} y+2 z x^{3} y+z^{2}+z^{2} x^{3}\right) \widetilde{c}_{2,3}-2 x y \widetilde{c}_{2,4}$

54) $s_{1}\left(x y \widetilde{c}_{1,3}\right)=-z x^{2} \widetilde{c}_{2,2}$

55) $s_{1}\left(x^{2} y \widetilde{c}_{1,3}\right)=(-1-z x) \widetilde{c}_{2,1}+\left(-z x^{3} y+z^{2} x^{3}\right) \widetilde{c}_{2,2}+z^{2} x^{3} \widetilde{c}_{2,3}-x y \widetilde{c}_{2,4}$

56) $s_{1}\left(x^{3} y \widetilde{c}_{1,3}\right)=-z \widetilde{c}_{2,2}$

57) $s_{1}\left(z \widetilde{c}_{1,3}\right)=0$

58) $s_{1}\left(z x \widetilde{c}_{1,3}\right)=z^{2} x^{3} y \widetilde{c}_{2,1}-\widetilde{c}_{2,2}+z x \widetilde{c}_{2,3}-\widetilde{c}_{2,4}$ 
59) $s_{1}\left(z x^{2} \widetilde{c}_{1,3}\right)=z x^{2} \widetilde{c}_{2,3}$

60) $s_{1}\left(z x^{3} \widetilde{c}_{1,3}\right)=\left(-1+2 x y+z x-z x^{2}+z x^{3} y-z^{2}+z^{2} x y+2 z^{2} x^{3} y\right) \widetilde{c}_{2,1}+\left(-2+x-x^{2}-\right.$ $\left.-2 z x^{2}+z x^{3}-2 z^{2}+z^{2} x^{3}\right) \widetilde{c}_{2,2}+\left(-1+x+2 z x-z x^{2}+z x^{3}-z x^{3} y-z^{2}+z^{2} x^{3}\right) \widetilde{c}_{2,3}+(-2+x y) \widetilde{c}_{2,4}$

61) $s_{1}\left(z y \widetilde{c}_{1,3}\right)=\left(1-2 x-x^{3}+y+x^{2} y+z x+z x^{3}-z y-z x^{3} y-z^{2}-z^{2} x^{2}+z^{2} x^{3}-\right.$ $\left.-z^{2} y+z^{2} x y\right) \widetilde{c}_{2,1}+\left(-x+y+x^{2} y-x^{3} y-z+z x+z x^{3}-z^{2} x^{3}-z^{2} y+z^{2} x y-z^{2} x^{2} y\right) \widetilde{c}_{2,2}+$ $+\left(-x+y+x^{2} y-x^{3} y-z-z x^{2}+z x^{3}+z y-z x^{3} y-z^{2} x^{3}-z^{2} y+z^{2} x y-z^{2} x^{2} y\right) \widetilde{c}_{2,3}+$ $+(1-x+y+x y) \widetilde{c}_{2,4}$

62) $s_{1}\left(z x y \widetilde{c}_{1,3}\right)=\left(-1-y-z x^{2}-z x^{2} y-z^{2} x+z^{2} y+z^{2} x^{2} y+z^{2} x^{3} y\right) \widetilde{c}_{2,1}+(-1-$ $\left.-x y-z x^{2}-z y+z x y-z x^{2} y+z^{2} x^{3}+z^{2} x^{2} y\right) \widetilde{c}_{2,2}+\left(-1-x y+z x+z x y-z x^{2} y+\right.$ $\left.+z x^{3} y+z^{2} x^{3}+z^{2} x^{2} y\right) \widetilde{c}_{2,3}+(-1-x-y-x y) \widetilde{c}_{2,4}$

63) $s_{1}\left(z x^{2} y \widetilde{c}_{1,3}\right)=\left(1-x-x^{3}+y+x^{2} y+z x+z x^{3}-z y-z^{2}+z^{2} x-z^{2} x^{2}-z^{2} y+\right.$ $\left.+z^{2} x y\right) \widetilde{c}_{2,1}+\left(y+x^{2} y-x^{3} y-z+z x+z x^{3}+z x^{2} y-z^{2} x^{3}-z^{2} y+z^{2} x y-z^{2} x^{2} y\right) \widetilde{c}_{2,2}+$ $+\left(y+x^{2} y-x^{3} y-z-z x^{2}+z x^{3}+z x^{2} y-z x^{3} y-z^{2} x^{3}-z^{2} y+z^{2} x y-z^{2} x^{2} y\right) \widetilde{c}_{2,3}+(1+y+x y) \widetilde{c}_{2,4}$

64) $s_{1}\left(z x^{3} y \widetilde{c}_{1,3}\right)=\left(-1+x+x^{3}-y-x^{2} y-z x-z x^{3}+z y+z^{2}+z^{2} x^{2}+z^{2} y-\right.$ $\left.-z^{2} x y\right) \widetilde{c}_{2,1}+\left(-y-x^{2} y+x^{3} y+z-z x-z x^{3}+z^{2} x^{3}+z^{2} y-z^{2} x y+z^{2} x^{2} y\right) \widetilde{c}_{2,2}+(-y-$ $\left.-x^{2} y+x^{3} y+z+z x^{2}-z x^{3}+z x^{3} y+z^{2} x^{3}+z^{2} y-z^{2} x y+z^{2} x^{2} y\right) \widetilde{c}_{2,3}+(-1-y-x y) \widetilde{c}_{2,4}$

65) $s_{1}\left(z^{2} \widetilde{c}_{1,3}\right)=\left(-x y-z x-z^{2} x^{3} y\right) \widetilde{c}_{2,1}+\left(1+z x^{2}+z^{2}\right) \widetilde{c}_{2,2}+\left(-z x+z x^{3} y+z^{2}\right) \widetilde{c}_{2,3}+$ $+(1-x y) \widetilde{c}_{2,4}$

66) $s_{1}\left(z^{2} x \widetilde{c}_{1,3}\right)=0$

67) $s_{1}\left(z^{2} x^{2} \widetilde{c}_{1,3}\right)=\left(-y-x y-z x-z x^{3}+z y-z x^{3} y+z^{2}+z^{2} x^{3}-z^{2} x y-z^{2} x^{3} y\right) \widetilde{c}_{2,1}+$ $+\left(1-x+x^{2}-y-x^{2} y+x^{3} y+z+z x^{2}-z x^{3}-z x^{3} y+z^{2}+z^{2} x^{2} y\right) \widetilde{c}_{2,2}+(-x-y-$ $\left.-x^{2} y+x^{3} y-z x+z x^{2}-z x^{3}+z y+z^{2} x^{2} y\right) \widetilde{c}_{2,3}+(1-x-y-x y) \widetilde{c}_{2,4}$

68) $s_{1}\left(z^{2} x^{3} \widetilde{c}_{1,3}\right)=0$

69) $s_{1}\left(z^{2} y \widetilde{c}_{1,3}\right)=\left(x+x^{3}-y-x^{2} y-z x^{3}+z y+z^{2}+z^{2} x^{2}-z^{2} x y\right) \widetilde{c}_{2,1}+\left(-y-x^{2} y+\right.$ $\left.+x^{3} y+z-z x-z x^{3}+z^{2} y-z^{2} x y+z^{2} x^{2} y\right) \widetilde{c}_{2,2}+\left(-y-x^{2} y+x^{3} y+z+z x^{2}-z x^{3}+\right.$ $\left.+z^{2} y-z^{2} x y+z^{2} x^{2} y\right) \widetilde{c}_{2,3}+(-1-y) \widetilde{c}_{2,4}$ 
70) $s_{1}\left(z^{2} x y \widetilde{c}_{1,3}\right)=\left(-x-x^{3}+y+x y+x^{2} y+z x+z x^{3}-z y-z^{2}-z^{2} x^{2}-z^{2} y+\right.$ $\left.+z^{2} x y+z^{2} x^{3} y\right) \widetilde{c}_{2,1}+\left(-1-x^{2}+y+x^{2} y-x^{3} y-z+z x-z x^{2}+z x^{3}-z^{2}-z^{2} y+\right.$ $\left.+z^{2} x y-z^{2} x^{2} y\right) \widetilde{c}_{2,2}+\left(y+x^{2} y-x^{3} y-z+z x-z x^{2}+z x^{3}-z x^{3} y-z^{2} y+z^{2} x y--z^{2} x^{2} y\right) \widetilde{c}_{2,3}+$ $(y+x y) \widetilde{c}_{2,4}$

71) $s_{1}\left(z^{2} x^{2} y \widetilde{c}_{1,3}\right)=\left(-1+2 x+x^{3}-2 y+x y-x^{2} y-z x^{2}-z x^{3}+z y+z x^{3} y+z^{2}+\right.$ $\left.+z^{2} x^{2}+z^{2} y-z^{2} x y+z^{2} x^{3} y\right) \widetilde{c}_{2,1}+\left(-1+x-2 y-x^{2} y+x^{3} y+z-z x-z x^{2}-z x^{3}-\right.$ $\left.-z^{2}+z^{2} x^{3}+z^{2} y-z^{2} x y+2 z^{2} x^{2} y\right) \widetilde{c}_{2,2}+\left(-1+x-2 y-x^{2} y+x^{3} y+z+z x+z x^{2}-\right.$ $\left.-z x^{3}-z^{2}+z^{2} x^{3}+z^{2} y-z^{2} x y+2 z^{2} x^{2} y\right) \widetilde{c}_{2,3}+(-2-2 y) \widetilde{c}_{2,4}$

72) $s_{1}\left(z^{2} x^{3} y \widetilde{c}_{1,3}\right)=-\widetilde{c}_{2,2}$

73) $s_{1}\left(\widetilde{c}_{1,4}\right)=0$

74) $s_{1}\left(x \widetilde{c}_{1,4}\right)=-z x^{2} \widetilde{c}_{2,1}+x \widetilde{c}_{2,2}+\left(-1-z x^{2}\right) \widetilde{c}_{2,3}$

75) $s_{1}\left(x^{2} \widetilde{c}_{1,4}\right)=\left(-x-x^{3}+y+x y+x^{2} y+z x+z x^{3}-z y-z^{2}-z^{2} x^{2}-z^{2} y+z^{2} x y+\right.$ $\left.+z^{2} x^{3} y\right) \widetilde{c}_{2,1}+\left(-1+y+x^{2} y-x^{3} y-z+z x-z x^{2}+z x^{3}-z^{2}-z^{2} y+z^{2} x y-z^{2} x^{2} y\right) \widetilde{c}_{2,2}+$ $+\left(y+x^{2} y-x^{3} y-z+z x-z x^{2}+z x^{3}-z x^{3} y-z^{2} y+z^{2} x y-z^{2} x^{2} y\right) \widetilde{c}_{2,3}+(y+x y) \widetilde{c}_{2,4}$

76) $s_{1}\left(x^{3} \widetilde{c}_{1,4}\right)=\left(1-x y-z x+z x^{2}-z x^{3}+z y-z x y+z x^{2} y-z x^{3} y+z^{2}+z^{2} x+\right.$ $\left.+z^{2} x^{2}+z^{2} x^{3}-z^{2} y-z^{2} x y-z^{2} x^{2} y-2 z^{2} x^{3} y\right) \widetilde{c}_{2,1}+\left(2-x+x^{2}-y+x y-x^{2} y+x^{3} y+\right.$ $\left.+z-z x+2 z x^{2}-z x^{3}+z y-z x y+z x^{2} y-z x^{3} y+z^{2}-z^{2} x^{3}\right) \widetilde{c}_{2,2}+\left(1-x-x^{3}-y+\right.$ $\left.+x y-x^{2} y+x^{3} y+z-2 z x+z x^{2}-z x^{3}+z y-z x y+z x^{2} y-z x^{3} y-z^{2} x^{3}\right) \widetilde{c}_{2,3}+\widetilde{c}_{2,4}$

77) $s_{1}\left(y \widetilde{c}_{1,4}\right)=\left(-1-x y-z x-z x^{2} y-z^{2} x+z^{2} y\right) \widetilde{c}_{2,1}+\left(y-x y-z x^{2} y+z^{2}+z^{2} x^{3}\right) \widetilde{c}_{2,2}+$ $+\left(-x y-z x^{2} y+2 z x^{3} y+z^{2}+z^{2} x^{3}\right) \widetilde{c}_{2,3}-2 x y \widetilde{c}_{2,4}$

78) $s_{1}\left(x y \widetilde{c}_{1,4}\right)=\left(x+x^{3}-y-x^{2} y-z x-z x^{3}+z y+z^{2}+z^{2} x^{2}-z^{2} x y-z^{2} x^{3} y\right) \widetilde{c}_{2,1}+$ $+\left(1-y+x y-x^{2} y+x^{3} y+z-z x-z x^{3}+z^{2} y-z^{2} x y+z^{2} x^{2} y\right) \widetilde{c}_{2,2}+\left(-y-x^{2} y+x^{3} y+z-\right.$ $\left.z x+z x^{2}-z x^{3}+z^{2} y-z^{2} x y+z^{2} x^{2} y\right) \widetilde{c}_{2,3}-y \widetilde{c}_{2,4}$

79) $s_{1}\left(x^{2} y \widetilde{c}_{1,4}\right)=(-1-z x) \widetilde{c}_{2,1}+\left(-z x^{3} y+z^{2} x^{3}\right) \widetilde{c}_{2,2}+\left(-x^{2} y+z^{2} x^{3}\right) \widetilde{c}_{2,3}-x y \widetilde{c}_{2,4}$

80) $s_{1}\left(x^{3} y \widetilde{c}_{1,4}\right)=\left(2 x+x^{3}-2 y-x y-x^{2} y-z x-2 z x^{3}+z y+2 z^{2}+z^{2} x^{2}+z^{2} y-2 z^{2} x y-\right.$ $\left.-z^{2} x^{3} y\right) \widetilde{c}_{2,1}+\left(1+x^{2}-2 y-x^{2} y+2 x^{3} y+z-z x+z x^{2}-2 z x^{3}+z^{2}+z^{2} y-z^{2} x y+2 z^{2} x^{2} y\right) \widetilde{c}_{2,2}+$ $+\left(-2 y-x^{2} y+x^{3} y+z-z x+2 z x^{2}-2 z x^{3}+z x^{3} y+z^{2} y-z^{2} x y+2 z^{2} x^{2} y\right) \widetilde{c}_{2,3}+(-2 y-x y) \widetilde{c}_{2,4}$ 
81) $s_{1}\left(z \widetilde{c}_{1,4}\right)=0$

82) $s_{1}\left(z x \widetilde{c}_{1,4}\right)=-\widetilde{c}_{1,4}$

83) $s_{1}\left(z x^{2} \widetilde{c}_{1,4}\right)=0$

84) $s_{1}\left(z x^{3} \widetilde{c}_{1,4}\right)=\left(-1+x+x^{3}-y+x y-x^{2} y-z x^{2}-z x^{3}+z y+z x^{3} y+z^{2} x^{2}+z^{2} y-z^{2} x y+\right.$ $\left.+z^{2} x^{3} y\right) \widetilde{c}_{2,1}+\left(-1+x-y-x^{2} y+x^{3} y+z-z x-z x^{2}-z^{2}+z^{2} x^{3}+z^{2} y-z^{2} x y+z^{2} x^{2} y\right) \widetilde{c}_{2,2}+$ $+\left(-1+x-y-x^{2} y+x^{3} y+z+z x-z x^{3}-z^{2}+z^{2} x^{3}+z^{2} y-z^{2} x y+z^{2} x^{2} y\right) \widetilde{c}_{2,3}+(-2-y) \widetilde{c}_{2,4}$

85) $s_{1}\left(z y \widetilde{c}_{1,4}\right)=\left(1-2 x-x^{3}+y+x^{2} y+z x+z x^{3}-z y-z x^{3} y-z^{2}-z^{2} x^{2}-z^{2} y+\right.$ $\left.+z^{2} x y\right) \widetilde{c}_{2,1}+\left(-x+y+x^{2} y-x^{3} y-z+z x+z x^{3}-z^{2} x^{3}-z^{2} y+z^{2} x y-z^{2} x^{2} y\right) \widetilde{c}_{2,2}+(-x+$ $\left.+y+x^{2} y-x^{3} y-z-z x^{2}+z x^{3}-z x^{3} y-z^{2} x^{3}-z^{2} y+z^{2} x y-z^{2} x^{2} y\right) \widetilde{c}_{2,3}+(1-x+y+x y) \widetilde{c}_{2,4}$

86) $s_{1}\left(z x y \widetilde{c}_{1,4}\right)=\left(-1-x y-z x-z x^{2} y-z^{2} x+z^{2} y\right) \widetilde{c}_{2,1}+\left(y-x y+z x y-z x^{2} y+\right.$ $\left.+z^{2}+z^{2} x^{3}\right) \widetilde{c}_{2,2}+\left(y-x y-z x^{2} y+2 z x^{3} y+z^{2}+z^{2} x^{3}\right) \widetilde{c}_{2,3}-2 x y \widetilde{c}_{2,4}$

87) $s_{1}\left(z x^{2} y \widetilde{c}_{1,4}\right)=\left(1-x-x^{3}+y+x^{2} y+z x+z x^{3}-z y-z^{2}-z^{2} x^{2}-z^{2} y+\right.$ $\left.+z^{2} x y\right) \widetilde{c}_{2,1}+\left(y+x^{2} y-x^{3} y-z+z x+z x^{3}+z x^{2} y-z^{2} x^{3}-z^{2} y+z^{2} x y-z^{2} x^{2} y\right) \widetilde{c}_{2,2}+(y+$ $\left.+x^{2} y-x^{3} y-z-z x^{2}+z x^{3}-z x^{3} y-z^{2} x^{3}-z^{2} y+z^{2} x y-z^{2} x^{2} y\right) \widetilde{c}_{2,3}+(1+y+x y) \widetilde{c}_{2,4}$

88) $s_{1}\left(z x^{3} y \widetilde{c}_{1,4}\right)=(-1-z x) \widetilde{c}_{2,1}+z^{2} x^{3} \widetilde{c}_{2,2}+z^{2} x^{3} \widetilde{c}_{2,3}-x y \widetilde{c}_{2,4}$

89) $s_{1}\left(z^{2} \widetilde{c}_{1,4}\right)=\left(x+x^{3}-y-x y-x^{2} y-z x-z x^{3}+z y+z^{2}+z^{2} x^{2}+z^{2} y-z^{2} x y-\right.$ $\left.-z^{2} x^{3} y\right) \widetilde{c}_{2,1}+\left(1-y-x^{2} y+x^{3} y+z-z x+z x^{2}-z x^{3}+z^{2}+z^{2} y-z^{2} x y+z^{2} x^{2} y\right) \widetilde{c}_{2,2}+$ $+\left(-y-x^{2} y+x^{3} y+z-z x+z x^{2}-z x^{3}+z x^{3} y+z^{2} y-z^{2} x y+z^{2} x^{2} y\right) \widetilde{c}_{2,3}+(-y-x y) \widetilde{c}_{2,4}$

90) $s_{1}\left(z^{2} x \widetilde{c}_{1,4}\right)=\left(-2-x^{2}+2 x y-z x^{2}+z x^{3} y-z^{2}+z^{2} x y+2 z^{2} x^{3} y\right) \widetilde{c}_{2,1}+(-2+x-$ $\left.-x^{2}-2 z x^{2}+z x^{3}-2 z^{2}+z^{2} x+2 z^{2} x^{3}\right) \widetilde{c}_{2,2}+\left(-1+x+2 z x-z x^{2}+z x^{3}-z^{2}+2 z^{2} x^{3}\right) \widetilde{c}_{2,3}-2 \widetilde{c}_{2,4}$

91) $s_{1}\left(z^{2} x^{2} \widetilde{c}_{1,4}\right)=\left(-y-x y-z x-z x^{3}+z y-z x^{3} y+z^{2}+z^{2} x^{3}-z^{2} x y-z^{2} x^{3} y\right) \widetilde{c}_{2,1}+$ $+\left(1-x+x^{2}-y-x^{2} y+x^{3} y+z+z x^{2}-z x^{3}-z x^{3} y+z^{2}+z^{2} x^{2} y\right) \widetilde{c}_{2,2}+(-x-y-$ $\left.-x^{2} y+x^{3} y-z x+z x^{2}-z x^{3}+z y-z^{2} x^{2}+z^{2} x^{2} y\right) \widetilde{c}_{2,3}+(1-x-y-x y) \widetilde{c}_{2,4}$

92) $s_{1}\left(z^{2} x^{3} \widetilde{c}_{1,4}\right)=\left(-1+z^{2} x^{3} y\right) \widetilde{c}_{2,1}+\left(-1+z^{2} x^{3}\right) \widetilde{c}_{2,2}+z x \widetilde{c}_{2,3}-\widetilde{c}_{2,4}$

93) $s_{1}\left(z^{2} y \widetilde{c}_{1,4}\right)=\left(x+x y-x^{2} y+z x+z x^{3} y-z^{2} x^{3}+z^{2} x^{3} y\right) \widetilde{c}_{2,1}+\left(-1+x-x^{2}-\right.$ $\left.-z x^{2}+z x^{3} y-z^{2}+z^{2} y-z^{2} x y\right) \widetilde{c}_{2,2}+\left(x+z x-z y-z^{2} x y\right) \widetilde{c}_{2,3}+(-1+x+x y) \widetilde{c}_{2,4}$ 
94) $s_{1}\left(z^{2} x y \widetilde{c}_{1,4}\right)=\left(1-2 x-x^{3}+y+x^{2} y+z x+z x^{3}-z y-z x^{3} y-z^{2}-z^{2} x^{2}+z^{2} x^{3}-z^{2} y+\right.$ $\left.+z^{2} x y\right) \widetilde{c}_{2,1}+\left(-x+y+x^{2} y-x^{3} y-z+z x+z x^{3}-z^{2} x^{3}-z^{2} y+2 z^{2} x y-z^{2} x^{2} y\right) \widetilde{c}_{2,2}+(-x+y+$ $\left.+x^{2} y-x^{3} y-z-z x^{2}+z x^{3}+z y-z x^{3} y-z^{2} x^{3}-z^{2} y+z^{2} x y-z^{2} x^{2} y\right) \widetilde{c}_{2,3}+(1-x+y+x y) \widetilde{c}_{2,4}$

95) $s_{1}\left(z^{2} x^{2} y \widetilde{c}_{1,4}\right)=\left(-y+x y+z x+z^{2} x^{3} y\right) \widetilde{c}_{2,1}+\left(-1-y-z x^{2}-z^{2}+z^{2} x^{2} y\right) \widetilde{c}_{2,2}+$ $+\left(-y+z x+z x^{2}-z x^{3} y-z^{2}\right) \widetilde{c}_{2,3}+(-1-y+x y) \widetilde{c}_{2,4}$

96) $s_{1}\left(z^{2} x^{3} y \widetilde{c}_{1,4}\right)=\left(1-x-x^{3}+y+x^{2} y+z x+z x^{3}-z y-z^{2}+z^{2} x-z^{2} x^{2}-z^{2} y+\right.$ $\left.+z^{2} x y\right) \widetilde{c}_{2,1}+\left(y+x^{2} y-x^{3} y-z+z x+z x^{3}+z x^{2} y-z^{2} x^{3}-z^{2} y+z^{2} x y-z^{2} x^{2} y+z^{2} x^{3} y\right) \widetilde{c}_{2,2}+$ $+\left(y+x^{2} y-x^{3} y-z-z x^{2}+z x^{3}+z x^{2} y-z x^{3} y-z^{2} x^{3}-z^{2} y+z^{2} x y-z^{2} x^{2} y\right) \widetilde{c}_{2,3}+(1+y+x y) \widetilde{c}_{2,4}$

- Definição da $s_{2}$ :

1) $s_{2}\left(g \widetilde{c}_{2,1}\right)= \begin{cases}\left(x y+x^{3} y+z y+z x^{2} y+z^{2} x+z^{2} x^{3}\right) \widetilde{c}_{3,1}, & \text { se } g=x^{3} y \\ \left(-x-x^{3}-y-x^{2} y-z x-z x^{3}-z x y-z x^{3} y-\right. & \\ \left.-z^{2} y-z^{2} x y-z^{2} x^{2} y-z^{2} x^{3} y\right) \widetilde{c}_{3,1}, & \text { se } g=z ; \\ 0, & \text { se } g \in P_{24} \text { e } g \neq x^{3} y, z .\end{cases}$

2) $s_{2}\left(g \widetilde{c}_{2,2}\right)=0$, para todo $g \in P_{24}$

3) $s_{2}\left(g \widetilde{c}_{2,3}\right)= \begin{cases}\left(y+x^{2} y+z x+z x^{3}+z^{2} x y+z^{2} x^{3} y\right) \widetilde{c}_{3,1}, & \text { se } g=z^{2} x^{3} y \\ 0, & \text { se } g \in P_{24} \text { e } g \neq z^{2} x^{3} y .\end{cases}$

4) $s_{2}\left(\widetilde{c}_{2,4}\right)=0$

5) $s_{2}\left(x \widetilde{c}_{2,4}\right)=0$

6) $s_{2}\left(x^{2} \widetilde{c}_{2,4}\right)=\left(-1-z x^{2}-z^{2}\right) \widetilde{c}_{3,1}$

7) $s_{2}\left(x^{3} \widetilde{c}_{2,4}\right)=\left(x^{3}+y+x^{2} y+z x+z x^{3}+z x y+z^{2} x y+z^{2} x^{2} y+z^{2} x^{3} y\right) \widetilde{c}_{3,1}$

8) $s_{2}\left(y \widetilde{c}_{2,4}\right)=0$

9) $s_{2}\left(x y \widetilde{c}_{2,4}\right)=0$

10) $s_{2}\left(x^{2} y \widetilde{c}_{2,4}\right)=\left(-y-z x^{3}-z^{2} x y\right) \widetilde{c}_{3,1}$

11) $s_{2}\left(x^{3} y \widetilde{c}_{2,4}\right)=\left(-x y-z x^{2} y-z^{2} x\right) \widetilde{c}_{3,1}$ 
12) $s_{2}\left(z \widetilde{c}_{2,4}\right)=\left(-1-x^{2}-z x^{2}-z^{2}\right) \widetilde{c}_{3,1}$

13) $s_{2}\left(z x \widetilde{c}_{2,4}\right)=\left(-y-x^{2} y-z x^{3}-z^{2} x y\right) \widetilde{c}_{3,1}$

14) $s_{2}\left(z x^{2} \widetilde{c}_{2,4}\right)=-\widetilde{c}_{3,1}$

15) $s_{2}\left(z x^{3} \widetilde{c}_{2,4}\right)=-y \widetilde{c}_{3,1}$

16) $s_{2}\left(z y \widetilde{c}_{2,4}\right)=\left(z y+z^{2} x^{3}\right) \widetilde{c}_{3,1}$

17) $s_{2}\left(z x y \widetilde{c}_{2,4}\right)=\left(y+x^{2} y+z x+z x^{3}+z x y+z^{2} x y+z^{2} x^{2} y+z^{2} x^{3} y\right) \widetilde{c}_{3,1}$

18) $s_{2}\left(z x^{2} y \widetilde{c}_{2,4}\right)=-x y \widetilde{c}_{3,1}$

19) $s_{2}\left(z x^{3} y \widetilde{c}_{2,4}\right)=-x \widetilde{c}_{3,1}$

20) $s_{2}\left(z^{2} \widetilde{c}_{2,4}\right)=\left(-1-z x^{2}\right) \widetilde{c}_{3,1}$

21) $s_{2}\left(z^{2} x \widetilde{c}_{2,4}\right)=\left(-x y-z x^{2} y\right) \widetilde{c}_{3,1}$

22) $s_{2}\left(z^{2} x^{2} \widetilde{c}_{2,4}\right)=z^{2} x^{2} \widetilde{c}_{3,1}$

23) $s_{2}\left(z^{2} x^{3} \widetilde{c}_{2,4}\right)=z^{2} x^{3} \widetilde{c}_{3,1}$

24) $s_{2}\left(z^{2} y \widetilde{c}_{2,4}\right)=\left(-x-z x^{3} y\right) \widetilde{c}_{3,1}$

25) $s_{2}\left(z^{2} x y \widetilde{c}_{2,4}\right)=\left(-y-z x^{3}\right) \widetilde{c}_{3,1}$

26) $s_{2}\left(z^{2} x^{2} y \widetilde{c}_{2,4}\right)=z^{2} x^{2} y \widetilde{c}_{3,1}$

27) $s_{2}\left(z^{2} x^{3} y \widetilde{c}_{2,4}\right)=\left(-y-x^{2} y-z x-z x^{3}-z^{2} x y\right) \widetilde{c}_{3,1}$

- Definição da s3:

$s_{3}\left(g \widetilde{c}_{3,1}\right)= \begin{cases}\widetilde{c}_{4,1}, & \text { se } g=z ; \\ 0, & \text { se } g \in P_{24} \quad \text { e } g \neq z .\end{cases}$

As homotopias $s_{n}, n \geq 4$, são definidas por extensões periódicas, ou seja, $s_{j}=s_{j+4}$, para todo $j \in \mathbb{N}$.

Demonstração. A prova será omitida, já que envolve contas simples, porém muito extensas. 
Basicamente consiste em verificar se cada uma das homotopias encontradas satisfazem as seguintes equações: $\partial_{1} s_{0}+s_{-1} \varepsilon=1_{C_{0}}$ e $\partial_{n+1} s_{n}+s_{n-1} \partial_{n}=1_{C_{n}}$, para todo $n>0$.

Como vimos, usando a resolução 4-periódica, definimos uma homotopia contrátil. Em trabalhos futuros temos como objetivo, através desta homotopia, construir aplicações diagonais para calcularmos a estrutura de anel dos grupos de cohomologia das formas espaciais esféricas tetraedrais, com vários coeficientes.

A seguir, faremos um resumo do método para o cálculo do produto cup, através da homotopia contrátil.

Dada uma resolução $C \stackrel{\varepsilon}{\rightarrow} \mathbb{Z}$ de $\mathbb{Z}$ sobre $\mathbb{R}$. O complexo de cadeias $C \otimes C$ é um $R$-módulo à esquerda, via ação diagonal $g \cdot(x \otimes y)=g x \otimes g y$, para todo $g \in G, x \in C_{i}$ e $y \in C_{j}$, o qual é estendido por linearidade para todo elemento de $R$.

Definição 4.5.7 A aplicação diagonal é um $R$-homomorfismo de cadeias $\Delta: C \rightarrow C \otimes C$ tal que o diagrama abaixo comuta

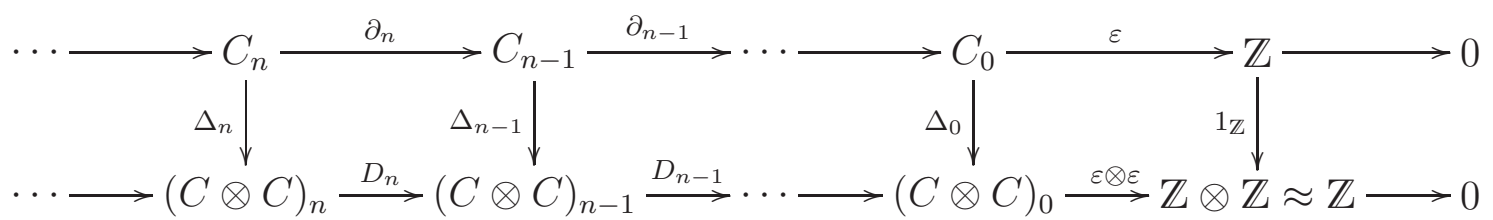

Para uma resolução livre de $C$, uma homotopia contrátil $s$ pode ser usada para construir uma aplicação diagonal $\Delta$.

Definição 4.5.8 A homotopia contrátil $\widetilde{s}$ para $C \otimes C$ é definida da seguinte forma:

$$
\begin{aligned}
& \widetilde{s}_{-1}=s_{-1} \otimes s_{-1}, \\
& \widetilde{s}_{n}\left(\sum_{i=0}^{n}\left(u_{i} \otimes v_{n-i}\right)\right)=\sum_{i=1}^{n} s_{i} u_{i} \otimes v_{n-i}+s_{-1} \varepsilon\left(u_{0}\right) \otimes s_{n}\left(v_{n}\right), n \geq 0,
\end{aligned}
$$

onde $u_{i} \in C_{i}$ e $v_{n-i} \in C_{n-i}$.

Dessa forma, definimos $\Delta_{n}: C \rightarrow C \otimes C$ em cada gerador $c_{j}$ de $C_{n}$ por

$$
\begin{aligned}
& \Delta_{0}=s_{-1} \varepsilon \otimes s_{-1} \varepsilon \\
& \Delta_{n}\left(c_{j}\right)=\widetilde{s}_{n-1} \Delta_{n-1} \partial_{n}\left(c_{j}\right),
\end{aligned}
$$


e é estendida para todo elemento de $C_{n}$ por $R$-linearidade.

Usando a aplicação diagonal acima, definimos uma operação multiplicativa no conjunto dos grupos de cohomologia $H^{*}(G ; A)$, onde $A$ é uma $R$-álgebra.

Definição 4.5.9 Seja $C \stackrel{\varepsilon}{\rightarrow} \mathbb{Z}$ uma resolução de $\mathbb{Z}$ sobre o anel grupo $R=\mathbb{Z} G$ do grupo $G$, $\Delta: C \rightarrow C \otimes C$ uma aplicação diagonal, e $A$ uma $R$-álgebra. Suponha que $\alpha \in H^{p}(G ; A)$ e $\beta \in H^{q}(G ; A)$, e são representados por u e v, respectivamente (isto é, $\alpha=[u]$ e $\beta=[v]$ ). Então, o produto cup de $\alpha$ e $\beta$ é definido como sendo o elemento $[\mu(u \otimes v) \Delta] \in H^{p+q}(G ; A)$, onde $\mu: A \otimes A \rightarrow A$ é a multiplicação em $A$.

$O$ produto cup de $\alpha$ e $\beta$ é denotado por $\alpha \cup \beta$.

A definição anterior é equivalente a Definição 4.2.1, vista no início do capítulo. 


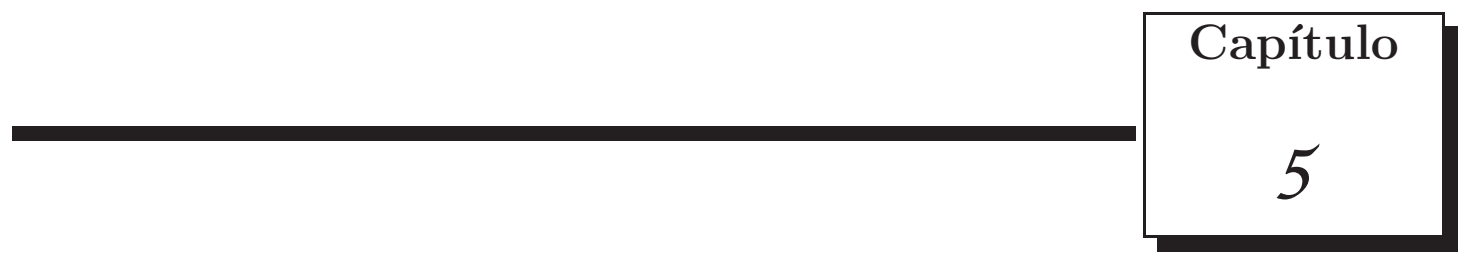

\section{Cálculo da torção de Reidemeister}

O objetivo deste capítulo é apresentar o cálculo da torção de Reidemeister para as formas espaciais esféricas tetraedrais, utilizando uma dada representação do seu grupo fundamental que defina um complexo que seja acíclico. Para um melhor entendimento do capítulo, apresentamos inicialmente as definições básicas envolvidas. Este capítulo é essencialmente baseado em [1, [2], 7] e [17.

\subsection{Grupo de Whitehead}

Seja $R$ um anel associativo com unidade. O grupo (aditivo) de todas as matrizes $n \times n$ não singulares sobre $R$ será denotado por $G L(n, R)$ (por vezes usaremos uma estrutura de anel). Identificamos cada $M \in G L(n, R)$ com a matriz

$$
\left(\begin{array}{cc}
M & 0 \\
0 & 1
\end{array}\right) \in G L(n+1, R)
$$

e obtemos inclusões $G L(1, R) \subset G L(2, R) \subset \cdots$. A união é chamada grupo linear geral infinito, denotado por $G L(R)$.

Uma matriz é chamada elementar se coincide com a identidade exceto para um único elemento fora da diagonal principal. 
Lema 5.1.1 ([15], Lema 1.1) O subgrupo $E(R) \subset G L(R)$, gerado por todas as matrizes elementares, é igual ao subgrupo comutador de $G L(R)$.

Segue que, $E(R)$ é um subgrupo normal de $G L(R)$, com grupo quociente comutativo. O quociente será chamado de grupo de Whitehead

$$
K_{1}(R)=G L(R) / E(R) .
$$

Geralmente, pensaremos em $K_{1}(R)$ como um grupo aditivo.

Observação 5.1.2 As classes do quociente $G L(R) / E(R)$ são as classes da relação de equivalência: $A \sim B$ se, e somente se, existem matrizes elementares $E_{1}, E_{2} \in E(R)$ tais que $A=E_{1} B E_{2}$.

Se o anel $R$ for comutativo, denotando por $R^{\times}$as unidades de $R$, a função determinante det $: K_{1}(R) \rightarrow R^{\times},[A] \mapsto \operatorname{det}(A)$, é um homomorfismo de grupos bem definido, desde que transformações elementares não modifiquem o determinante de uma matriz, ou seja, $\operatorname{det}(A E)=\operatorname{det}(A) \operatorname{det}(E)=\operatorname{det}(A)$, pois $\operatorname{det}(E)=1$. A imagem está em $R^{\times}$, pois as matrizes do grupo linear geral $G L(R)$ são invertíveis. Considerando o grupo linear especial $S L(R)$, consistindo de todas as matrizes em $G L(R)$ com determinante 1, podemos definir o quociente $S L(R) / E(R)$, denotado por $S K_{1}(R)$ e chamado de grupo de Whitehead especial.

Notemos a decomposição em soma direta

$$
K_{1}(R) \simeq R^{\times} \oplus S K_{1}(R)
$$

devido à inclusão $R^{\times} \subset G L(1, R) \subset G L(R)$ e à sequência exata curta

$$
0 \longrightarrow S K_{1}(R) \stackrel{i}{\longrightarrow} K_{1}(R) \stackrel{\text { det }}{\longrightarrow} R^{\times} \longrightarrow 0 .
$$

Exemplo 5.1.3 Se $\mathbb{F}$ é um corpo, então $K_{1}(\mathbb{F}) \simeq \mathbb{F}^{\times}=\mathbb{F}-\{0\}$. Se $\mathbb{F}$ é um anel de divisão, então $K_{1}(\mathbb{F})$ pode ser identificado com o grupo abelianizado $\mathbb{F}^{\times} /\left[\mathbb{F}^{\times}, \mathbb{F}^{\times}\right]$.

Definição 5.1.4 Seja $[-1] \in K_{1}(R)$ o elemento de ordem 2 correspondendo à unidade 
$(-1) \in G L(1, R) \subset G L(R)$. O quociente

$$
\widetilde{K}_{1}(R)=K_{1}(R) /\{[1],[-1]\}
$$

será chamadd1 de grupo de Whitehead reduzido de $R$.

A vantagem de passar para tal quociente é que duas matrizes $A, B$ que diferem somente por uma permutação de colunas (ou linhas) representam o mesmo elemento em $\widetilde{K}_{1}(R)$.

Dois exemplos importantes:

1. O grupo de Whitehead do grupo trivial é trivial. De fato, como o anel de grupos inteiro do grupo trivial é $\mathbb{Z}$, toda matriz pode ser escrita como o produto de uma matriz diagonal com um produto de matrizes elementares.

2. Para os números reais $\mathbb{R}, \widetilde{K}_{1}(\mathbb{R})$ é isomorfo ao grupo multiplicativo $\mathbb{R}^{+}$dos números reais positivos. Um isomorfismo específico é dado pela correspondência $\left(a_{i j}\right) \mapsto\left|\operatorname{det}\left(a_{i j}\right)\right|$.

Se $R$ é comutativo, o homomorfismo determinante det $: K_{1}(R) \rightarrow R^{\times}$definido anteriormente para o quociente (também denotado por det) det : $\widetilde{K}_{1}(R) \rightarrow R^{\times} /\{ \pm 1\}$, ou seja, se $A= \pm B E$ então $\operatorname{det}(A)= \pm \operatorname{det}(B)$, pois matrizes elementares possuem determinante 1. Assim sendo, $\operatorname{det}[A]=\operatorname{det}[B] \in R^{\times} /\{ \pm 1\}$.

\section{2 Álgebra dos quatérnios}

Nesta seção apresentamos apenas alguns resultados para a álgebra dos quatérnios que serão úteis futuramente. Para maiores detalhes sobre o assunto e para as provas omitidas, sugerimos o trabalho de H. Aslaksen [2] e as referências lá citadas, ou [1].

Os quatérnios, descobertos por William Rowan Hamilton em 1843, formam uma álgebra associativa não comutativa sobre $\mathbb{R}$,

$$
\mathbb{H}=\{a+b i+c j+d k ; a, b, c, d \in \mathbb{R}\},
$$

\footnotetext{
${ }^{1}$ Em alguns casos não comutativos pode acontecer de $[-1]=[1]$ de modo que $\widetilde{K}_{1}(R)=K_{1}(R)$.
} 
onde

$$
i j=k=-j i, \quad j k=i=-k j, \quad k i=j=-i k, \quad i^{2}=j^{2}=k^{2}=-1 .
$$

Podemos escrever $z \in \mathbb{H}$ na forma $z=x+y j, x, y \in \mathbb{C}$ mas, precisamos lembrar que $j y=\bar{y} j$, para $y \in \mathbb{C}$. Notemos que $\mathbb{H}$ não é uma álgebra sobre $\mathbb{C}$, já que o centro de $\mathbb{H}$ é somente $\mathbb{R}$. A conjugação em $\mathbb{H}$ é definida por $\overline{a+b i+c j+d k}=a-b i-c j-d k$ e satisfaz $\overline{u v}=\bar{v} \bar{u}$. Os quatérnios da forma $b i+c j+d k$, com $b, c, d \in \mathbb{R}$, serão chamados de quatérnios puros.

O seguinte lema nos diz que esta álgebra é isomorfa a uma álgebra de $M(2, \mathbb{C})$.

Lema 5.2.1 Para cada quatérnio $q=a+b i+c j+d k$, definimos $A(q) \in M(2, \mathbb{C})$ por

$$
A(q)=\left(\begin{array}{cc}
a+b i & c+d i \\
-c+d i & a-b i
\end{array}\right)
$$

Então a aplicação $q \mapsto A(q)$ é injetora e $A\left(q_{1} q_{2}\right)=A\left(q_{1}\right) A\left(q_{2}\right)$.

Definimos a norma (Euclidiana) de um quatérnio por

$$
|q|^{2}=q \bar{q}=a^{2}+b^{2}+c^{2}+d^{2} \quad(q=a+b i+c j+d k) .
$$

Segue que $|q|^{2}=|\bar{q}|^{2}$ e se $|q|^{2} \neq 0$, isto é, $q \neq 0$, então $q\left(\bar{q} /|q|^{2}\right)=1$, de modo que cada quatérnio não nulo possui um inverso multiplicativo $q^{-1}=\bar{q} /|q|^{2}$. Enunciamos então o seguinte resultado:

Lema 5.2.2 A álgebra $\mathbb{H}$ dos quatérnios é uma álgebra de divisão, isto é, para cada quatérnio não nulo q existe um inverso (à direita e à esquerda) $q^{-1}$.

Das igualdades (5.2.1) e (5.2.2) é imediato que, para todo quatérnio $q,|q|^{2}=\operatorname{det} A(q)$ e portanto

$$
\left|q_{1} q_{2}\right|^{2}=\operatorname{det} A\left(q_{1} q_{2}\right)=\operatorname{det}\left(A\left(q_{1}\right) A\left(q_{2}\right)\right)=\operatorname{det} A\left(q_{1}\right) \operatorname{det} A\left(q_{2}\right)=\left|q_{1}\right|^{2}\left|q_{2}\right|^{2} .
$$

Pela equação (5.2.3) acima deduzimos que o conjunto dos quatérnios de norma 1 forma um grupo multiplicativo, denotado por $\mathbb{H}_{1}$. Da equação (5.2.2) segue que para $q \in \mathbb{H}_{1}$, 
$q^{-1}=\bar{q}$. Se pensarmos em $\mathbb{H}$ como um espaço 4-dimensional com coordenadas $a, b, c, d$ então $\mathbb{H}_{1}$ é simplesmente a 3-esfera em $\mathbb{R}^{4}$.

Observação 5.2.3 Devido ao Exemplo 5.1.3 e ao Lema 5.2.2, como $\mathbb{H}$ é um anel de divisão, então $K_{1}(\mathbb{H})$ pode ser identificado com o grupo abelianizado $\mathbb{H}^{\times} /\left[\mathbb{H}^{\times}, \mathbb{H}^{\times}\right]$. Notemos que, $\mathrm{H}^{\times} /\left[\mathrm{H}^{\times}, \mathrm{H}^{\times}\right]$é isomorfo ao conjunto dos números reais positivos via a aplicação $\varphi$, definida da seguinte forma

$$
\begin{gathered}
\varphi: \mathbb{H}^{\times} /\left[\mathbb{H}^{\times}, \mathbb{H}^{\times}\right] \longrightarrow \mathbb{R}^{+} \\
x\left[\mathbb{H}^{\times}, \mathbb{H}^{\times}\right] \mapsto\|x\| .
\end{gathered}
$$

\subsection{Torção de Reidemeister de um complexo de cadeias}

Seja $R$ um anel associativo com unidade.

Assumimos que $R$ possui um número de base invariante, ou seja, se $M$ é qualquer módulo livre, finitamente gerado sobre $R$, então quaisquer duas bases de $M$ possuem a mesma cardinalidade. Notemos que tal afirmação é satisfeita se $R=\mathbb{Z} G$ é o anel grupo de algum grupo $G$ (vide [7, Seção 9.2]), e se $R$ é um anel de divisão (vide [7, Seção 9.1]).

Todos os módulos considerados são $R$-módulos à esquerda finitamente gerados.

Seja $M$ um $R$-módulo livre de dimensão finita. Sejam $u=\left\{u_{1}, u_{2}, \ldots, u_{k}\right\}$ e $v=\left\{v_{1}, v_{2}, \ldots, v_{k}\right\}$ duas bases para $M$. Denotemos por $(v / u)$ a matriz quadrada de ordem $k$ não singular sobre $R$, definida como sendo a matriz $A$ de mudança de base, isto é, $(v / u)=A$, onde $v_{i}=\sum_{j=1}^{k} A_{i, j} u_{j}$.

Seja

$$
C: \quad C_{m} \stackrel{\partial_{m}}{\longrightarrow} C_{m-1} \stackrel{\partial_{m-1}}{\longrightarrow} \ldots \stackrel{\partial_{2}}{\longrightarrow} C_{1} \stackrel{\partial_{1}}{\longrightarrow} C_{0}
$$

o complexo de cadeias de $R$-módulos livres (de dimensão finita) e de comprimento $m$. Consideremos $Z_{q}=\operatorname{Ker} \partial_{q}, B_{q}=\operatorname{Im} \partial_{q+1}$ e $H_{q}(C)=Z_{q} / B_{q}$ os grupos de homologia de $C$. Afim de definirmos a torção de $C$, assumimos que $B_{q}$ é um $R$-módulo livre para cada $q$. Notemos que se $R$ é um anel de divisão (em particular um corpo), qualquer módulo sobre $R$ é livre, e cada submódulo de um módulo livre finitamente gerado, sobre um domínio de ideais principais é livre e finitamente gerado. Consideremos $H_{q}$ como um $R$-módulo livre 
para todo $q$.

Para cada $q$, fixemos uma base $c_{q}$ para $C_{q}$ e um conjunto de elementos linearmente independentes $h_{q}$ em $Z_{q}$, cuja projeção $\bar{h}_{q}$ é uma base para $H_{q}$. Seja $b_{q}$ um conjunto de elementos de $C_{q}$ tal que $\partial_{q}\left(b_{q}\right)$ é uma base para $B_{q-1}$ (claramente isso implica que $b_{q}$ é linearmente independente). Usando as inclusões $0 \subseteq Z_{q} \subseteq C_{q}$ e os isomorfismos $Z_{q} / B_{q} \simeq H_{q}$ e $C_{q} / Z_{q} \simeq B_{q-1}$, vemos que o conjunto de elementos $\left\{\partial_{q+1}\left(b_{q+1}\right), h_{q}, b_{q}\right\}$ é uma base para $C_{q}$.

Definição 5.3.1 (Torção de Reidemeister de um complexo de cadeias) Se o anel $R$ é comutativo, a torção de Reidemeister (ou $R$ torção) do complexo $C$, com relação à base graduada $\bar{h}=\left\{\bar{h}_{q}\right\}$ é

$$
\tau_{R}(C ; \bar{h})=\prod_{q=0}^{m}\left|\operatorname{det}\left(\partial_{q+1}\left(b_{q+1}\right), h_{q}, b_{q} / c_{q}\right)\right|^{(-1)^{q}} \in R^{\times} /\{ \pm 1\} .
$$

A torção $\tau_{R}(C ; \bar{h})$ não depende da escolha da base graduada $b=\left\{b_{q}\right\}$ (vide [17, pag.365]). Entretanto, $\tau_{R}(C ; \bar{h})$ depende da base graduada $\bar{h}=\left\{\bar{h}_{q}\right\}$ da homologia e não depende da escolha dos ciclos $h_{q} \in Z_{q}$. Para simplificar a notação escrevemos $\tau_{R}(C ; h)$ ao invés de $\tau_{R}(C ; \bar{h})$.

\subsection{Cálculo da torção de Reidemeister das formas es- paciais esféricas tetraedrais}

Quando definimos as formas espaciais esféricas tetraedrais, fizemos uso da representação complexa $\alpha: P_{24} \rightarrow U(2 n, \mathbb{C})$, definida no Lema 2.1.3, Como $\pi_{1}\left(\mathscr{P}_{3}\right) \simeq P_{24}$, faremos o cálculo da $R$ torção utilizando a seguinte representação em $U(2, \mathbb{C})$

$$
\rho: P_{24} \rightarrow \mathbb{H}^{\times} \stackrel{A}{\rightarrow} M(2, \mathbb{C}),
$$

onde $A$ é definida na equação (5.2.1) . Definimos então

$$
\rho(x)=A(i)=\left(\begin{array}{cc}
i & 0 \\
0 & -i
\end{array}\right), \quad \rho(y)=A(j)=\left(\begin{array}{cc}
0 & -1 \\
1 & 0
\end{array}\right) \quad \mathrm{e}
$$




$$
\rho(z)=A\left(-\frac{1}{2}-\frac{1}{2} i-\frac{1}{2} j-\frac{1}{2} k\right)=\left(\begin{array}{cc}
-\frac{1}{2}-\frac{1}{2} i & -\frac{1}{2}-\frac{1}{2} i \\
\frac{1}{2}-\frac{1}{2} i & -\frac{1}{2}+\frac{1}{2} i
\end{array}\right) .
$$

A representação acima induz um homomorfismo de anel $\rho: \mathbb{Z} P_{24} \rightarrow \mathbb{H}^{\times}$e, aplicando $\rho$ ao complexo de cadeias $C\left(\widetilde{\mathscr{P}}_{4 n-1} ; \mathbb{Z} P_{24}\right)$, obtemos o complexo

$$
C\left(\mathscr{P}_{4 n-1} ; \mathbb{H}_{\rho}\right)=\mathbb{H} \otimes_{\rho} C\left(\widetilde{P}_{4 n-1} ; \mathbb{Z} P_{24}\right)
$$

como visto abaixo:

$$
\begin{gathered}
\cdots \longrightarrow \mathbb{H}\left[\widetilde{c}_{4 q-1,1}\right] \stackrel{\partial_{4 q-1}}{\longrightarrow} \mathbb{H}\left[\widetilde{c}_{4 q-2,1}, \widetilde{c}_{4 q-2,2}, \widetilde{c}_{4 q-2,3}, \widetilde{c}_{4 q-2,4}\right] \stackrel{\partial_{4 q-2}}{\longrightarrow} \\
\stackrel{\partial_{4 q-2}}{\longrightarrow} \mathbb{H}\left[\widetilde{c}_{4 q-3,1}, \widetilde{c}_{4 q-3,2}, \widetilde{c}_{4 q-3,3}, \widetilde{c}_{4 q-3,4}\right] \stackrel{\partial_{4 q-3}}{\longrightarrow} \mathbb{H}\left[\widetilde{c}_{4 q-4,1}\right] \stackrel{\partial_{4 q-4}}{\longrightarrow} \cdots,
\end{gathered}
$$

com os operadores bordos dados por

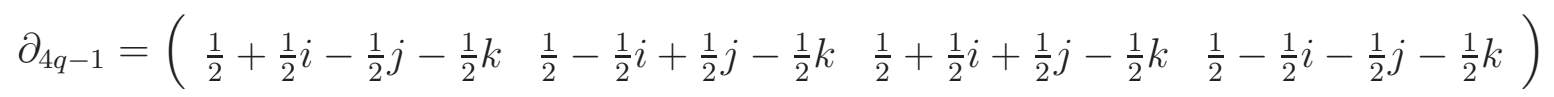

$$
\begin{aligned}
& \partial_{4 q-2}=\left(\begin{array}{cccc}
-\frac{1}{2}-\frac{1}{2} i+\frac{1}{2} j-\frac{1}{2} k & 1 & -1 & 0 \\
-1 & 0 & -\frac{1}{2}+\frac{1}{2} i-\frac{1}{2} j-\frac{1}{2} k & 1 \\
0 & -\frac{1}{2}-\frac{1}{2} i-\frac{1}{2} j-\frac{1}{2} k & 1 & -1 \\
1 & -1 & 0 & -\frac{1}{2}+\frac{1}{2} i+\frac{1}{2} j-\frac{1}{2} k
\end{array}\right) \\
& \partial_{4 q-3}=\left(\begin{array}{c}
-\frac{1}{2}-\frac{1}{2} i+\frac{1}{2} j+\frac{1}{2} k \\
-\frac{1}{2}-\frac{1}{2} i-\frac{1}{2} j+\frac{1}{2} k \\
-\frac{1}{2}+\frac{1}{2} i-\frac{1}{2} j+\frac{1}{2} k \\
-\frac{1}{2}+\frac{1}{2} i+\frac{1}{2} j+\frac{1}{2} k
\end{array}\right) \\
& \partial_{4 q-4}=(0) \text {. }
\end{aligned}
$$

O complexo anterior é um complexo de cadeias periódico de H-módulos livres e finitamente gerados. Como H é um anel de divisão, seus bordos e ciclos são livres e, dessa forma, a torção $\tau_{R}\left(\mathscr{P}_{4 n-1} ; h\right)$ está bem definida.

Podemos agora calcular a torção de Reidemeister das formas espaciais esféricas tetraedrais. 
Porém, antes disso, verifiquemos a exatidão do complexo de cadeias em questão.

Proposição 5.4.1 O complexo $C\left(\mathscr{P}_{4 n-1} ; \mathbb{H}_{\rho}\right)$ é acíclico.

Demonstração. O complexo visto anteriormente na equação (5.4.1) é semi-exato pela Afirmação 2.3.1, ou seja, $\operatorname{Im} \partial_{k}$ está contida no $\operatorname{Ker} \partial_{k-1}$, para $4 q-1 \leq k \leq 4 q-3$. Resta mostrar que $\operatorname{dim}\left(\operatorname{Im} \partial_{k}\right)$ é igual a $\operatorname{dim}\left(\operatorname{Ker} \partial_{k-1}\right)$.

- $\operatorname{Ker} \partial_{4 q-1}$

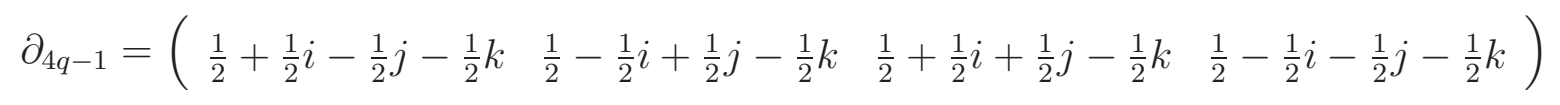

Seja uma $(4 q-1)$-cadeia representada por $d$ pertencente ao $K e r \partial_{4 q-1}$, então

$$
\begin{aligned}
& d\left(\frac{1}{2}+\frac{1}{2} i-\frac{1}{2} j-\frac{1}{2} k\right) \widetilde{c}_{4 q-2,1}=0 \\
& d\left(\frac{1}{2}-\frac{1}{2} i+\frac{1}{2} j-\frac{1}{2} k\right) \widetilde{c}_{4 q-2,2}=0 \\
& d\left(\frac{1}{2}+\frac{1}{2} i+\frac{1}{2} j-\frac{1}{2} k\right) \widetilde{c}_{4 q-2,3}=0 \\
& d\left(\frac{1}{2}-\frac{1}{2} i-\frac{1}{2} j-\frac{1}{2} k\right) \widetilde{c}_{4 q-2,4}=0
\end{aligned}
$$

Dessa forma, temos $d=0$.

Logo, $\operatorname{Ker} \partial_{4 q-1}=0$, ou seja, $\operatorname{dim}\left(\operatorname{Ker} \partial_{4 q-1}\right)=0$.

- $\operatorname{Im} \partial_{4 q-1}$

Como as linhas da matriz do $\partial_{4 q-1}$ são linearmente independentes, $\operatorname{dim}\left(\operatorname{Im} \partial_{4 q-1}\right)=1$.

- $K e r \partial_{4 q-2}$

$$
\partial_{4 q-2}=\left(\begin{array}{cccc}
-\frac{1}{2}-\frac{1}{2} i+\frac{1}{2} j-\frac{1}{2} k & 1 & -1 & 0 \\
-1 & 0 & -\frac{1}{2}+\frac{1}{2} i-\frac{1}{2} j-\frac{1}{2} k & 1 \\
0 & -\frac{1}{2}-\frac{1}{2} i-\frac{1}{2} j-\frac{1}{2} k & 1 & -1 \\
1 & -1 & 0 & -\frac{1}{2}+\frac{1}{2} i+\frac{1}{2} j-\frac{1}{2} k
\end{array}\right)
$$


Considere uma $(4 q-2)$-cadeia $\sigma$ representada por $(d, e, f, g)$, então:

$$
\begin{aligned}
\partial_{4 q-2}(\sigma) & =\partial_{4 q-2}\left(d \widetilde{c}_{4 q-2,1}+e \widetilde{c}_{4 q-2,2}+f \widetilde{c}_{4 q-2,3}+g \widetilde{c}_{4 q-2,4}\right) \\
& =d\left[\left(-\frac{1}{2}-\frac{1}{2} i+\frac{1}{2} j-\frac{1}{2} k\right) \widetilde{c}_{4 q-3,1}+\widetilde{c}_{4 q-3,2}-\widetilde{c}_{4 q-3,3}\right]+e\left[-\widetilde{c}_{4 q-3,1}+\left(-\frac{1}{2}+\right.\right. \\
& \left.\left.+\frac{1}{2} i-\frac{1}{2} j-\frac{1}{2} k\right) \widetilde{c}_{4 q-3,3}+\widetilde{c}_{4 q-3,4}\right]+f\left[\left(-\frac{1}{2}-\frac{1}{2} i-\frac{1}{2} j-\frac{1}{2} k\right) \widetilde{c}_{4 q-3,2}+\widetilde{c}_{4 q-3,3}-\right. \\
& \left.-\widetilde{c}_{4 q-3,4}\right]+g\left[\widetilde{c}_{4 q-3,1}-\widetilde{c}_{4 q-3,2}+\left(-\frac{1}{2}+\frac{1}{2} i+\frac{1}{2} j-\frac{1}{2} k\right) \widetilde{c}_{4 q-3,4}\right] \\
& =\left[d\left(-\frac{1}{2}-\frac{1}{2} i+\frac{1}{2} j-\frac{1}{2} k\right)-e+g\right] \widetilde{c}_{4 q-3,1}+\left[d+f\left(-\frac{1}{2}-\frac{1}{2} i-\frac{1}{2} j-\frac{1}{2} k\right)-\right. \\
& -g] \widetilde{c}_{4 q-3,2}+\left[-d+e\left(-\frac{1}{2}+\frac{1}{2} i-\frac{1}{2} j-\frac{1}{2} k\right)+f\right] \widetilde{c}_{4 q-3,3}+\left[e-f+g\left(-\frac{1}{2}+\right.\right. \\
& \left.\left.+\frac{1}{2} i+\frac{1}{2} j-\frac{1}{2} k\right)\right] \widetilde{c}_{4 q-3,4}
\end{aligned}
$$

A $(4 q-2)$-cadeia $\sigma$ pertence ao $K e r \partial_{4 q-2}$ se, e somente se,

$$
\left\{\begin{array}{l}
d\left(-\frac{1}{2}-\frac{1}{2} i+\frac{1}{2} j-\frac{1}{2} k\right)-e+g=0 \\
d+f\left(-\frac{1}{2}-\frac{1}{2} i-\frac{1}{2} j-\frac{1}{2} k\right)-g=0 \\
-d+e\left(-\frac{1}{2}+\frac{1}{2} i-\frac{1}{2} j-\frac{1}{2} k\right)+f=0 \\
e-f+g\left(-\frac{1}{2}+\frac{1}{2} i+\frac{1}{2} j-\frac{1}{2} k\right)=0
\end{array}\right.
$$

Pela quarta equação temos $f=e+g\left(-\frac{1}{2}+\frac{1}{2} i+\frac{1}{2} j-\frac{1}{2} k\right)$.

Substituindo essa igualdade na terceira equação, obtemos

$$
\begin{aligned}
& -d+e\left(-\frac{1}{2}+\frac{1}{2} i-\frac{1}{2} j-\frac{1}{2} k\right)+e+g\left(-\frac{1}{2}+\frac{1}{2} i+\frac{1}{2} j-\frac{1}{2} k\right)=0 \\
& d=e\left(\frac{1}{2}+\frac{1}{2} i-\frac{1}{2} j-\frac{1}{2} k\right)+g\left(-\frac{1}{2}+\frac{1}{2} i+\frac{1}{2} j-\frac{1}{2} k\right) .
\end{aligned}
$$

Agora, substituindo a igualdade encontrada para $f$ e $d$ na segunda equação, ficamos com

$$
\begin{gathered}
e\left(\frac{1}{2}+\frac{1}{2} i-\frac{1}{2} j-\frac{1}{2} k\right)+g\left(-\frac{1}{2}+\frac{1}{2} i+\frac{1}{2} j-\frac{1}{2} k\right)+e\left(-\frac{1}{2}-\frac{1}{2} i-\frac{1}{2} j-\frac{1}{2} k\right)+ \\
+g\left(\frac{1}{4}-\frac{1}{4} i-\frac{1}{4} j+\frac{1}{4} k+\frac{1}{4} i+\frac{1}{4}-\frac{1}{4} k-\frac{1}{4} j+\frac{1}{4} j+\frac{1}{4} k+\frac{1}{4}+\frac{1}{4} i+\frac{1}{4} k-\frac{1}{4} j+\frac{1}{4} i-\frac{1}{4}\right)-g=0
\end{gathered}
$$




$$
\begin{gathered}
e(-j-k)+g\left(-\frac{1}{2}+\frac{1}{2} i+\frac{1}{2} j-\frac{1}{2} k\right)+g\left(\frac{1}{2}+\frac{1}{2} i-\frac{1}{2} j+\frac{1}{2} k\right)-g=0 \\
e(-j-k)+g(-1+i)=0 \\
e=\frac{g(1-i)}{(-j-k)} .
\end{gathered}
$$

Dessa forma, temos

$$
\begin{gathered}
d=\frac{g(1-i)}{(-j-k)}\left(\frac{1}{2}+\frac{1}{2} i-\frac{1}{2} j-\frac{1}{2} k\right)+g\left(-\frac{1}{2}+\frac{1}{2} i+\frac{1}{2} j-\frac{1}{2} k\right) \\
d=\frac{g}{(-j-k)}\left(\frac{1}{2}+\frac{1}{2} i-\frac{1}{2} j-\frac{1}{2} k-\frac{1}{2} i+\frac{1}{2}+\frac{1}{2} k-\frac{1}{2} j\right)+g\left(-\frac{1}{2}+\frac{1}{2} i+\frac{1}{2} j-\frac{1}{2} k\right) \\
d=\frac{g}{(-j-k)}(1-j)+g\left(-\frac{1}{2}+\frac{1}{2} i+\frac{1}{2} j-\frac{1}{2} k\right) .
\end{gathered}
$$

Portanto,

$$
f=\frac{g(1-i)}{(-j-k)}+g\left(-\frac{1}{2}+\frac{1}{2} i+\frac{1}{2} j-\frac{1}{2} k\right) .
$$

Assim,

$$
\begin{aligned}
\sigma & =\left[\frac{g}{(-j-k)}(1-j)+g\left(-\frac{1}{2}+\frac{1}{2} i+\frac{1}{2} j-\frac{1}{2} k\right)\right] \widetilde{c}_{4 q-2,1}+\left[\frac{g(1-i)}{(-j-k)}\right] \widetilde{c}_{4 q-2,2}+ \\
& +\left[\frac{g(1-i)}{(-j-k)}+g\left(-\frac{1}{2}+\frac{1}{2} i+\frac{1}{2} j-\frac{1}{2} k\right)\right] \widetilde{c}_{4 q-2,3}+g \widetilde{c}_{4 q-2,4} \\
& =\left\{\left[\frac{1}{(-j-k)}(1-j)+\left(-\frac{1}{2}+\frac{1}{2} i+\frac{1}{2} j-\frac{1}{2} k\right)\right] \widetilde{c}_{4 q-2,1}+\left[\frac{(1-i)}{(-j-k)}\right] \widetilde{c}_{4 q-2,2}+\right. \\
& \left.+\left[\frac{(1-i)}{(-j-k)}+\left(-\frac{1}{2}+\frac{1}{2} i+\frac{1}{2} j-\frac{1}{2} k\right)\right] \widetilde{c}_{4 q-2,3}+\widetilde{c}_{4 q-2,4}\right\} g
\end{aligned}
$$

Logo, $\operatorname{dim}\left(\operatorname{Ker} \partial_{4 q-2}\right)=1$.

Portanto, como vimos que o complexo é semi-exato, a $\operatorname{Im} \partial_{4 q-1}$ está contida no $K e r \partial_{4 q-2}$, e pelos resultados obtidos acima, segue que a $\operatorname{dim}\left(\begin{array}{ll}\operatorname{Im} & \partial_{4 q-1}\end{array}\right)=$ $=\operatorname{dim}\left(\operatorname{Ker} \partial_{4 q-2}\right)=1$. Dessa forma, $\operatorname{Im} \partial_{4 q-1}=\operatorname{Ker} \partial_{4 q-2}$.

- $\operatorname{Im} \partial_{4 q-2}$ 
$\operatorname{Im} \partial_{4 q-2}=\left(\begin{array}{cccc}-\frac{1}{2}-\frac{1}{2} i+\frac{1}{2} j-\frac{1}{2} k & 1 & -1 & 0 \\ -1 & 0 & -\frac{1}{2}+\frac{1}{2} i-\frac{1}{2} j-\frac{1}{2} k & 1 \\ 0 & -\frac{1}{2}-\frac{1}{2} i-\frac{1}{2} j-\frac{1}{2} k & 1 & -1 \\ 1 & -1 & 0 & -\frac{1}{2}+\frac{1}{2} i+\frac{1}{2} j-\frac{1}{2} k\end{array}\right)$

Trocando a segunda linha com a terceira linha da matriz anterior, obtemos a seguinte matriz:

$$
\left(\begin{array}{cccc}
-\frac{1}{2}-\frac{1}{2} i+\frac{1}{2} j-\frac{1}{2} k & 1 & -1 & 0 \\
0 & -\frac{1}{2}-\frac{1}{2} i-\frac{1}{2} j-\frac{1}{2} k & 1 & -1 \\
-1 & 0 & -\frac{1}{2}+\frac{1}{2} i-\frac{1}{2} j-\frac{1}{2} k & 1 \\
1 & -1 & 0 & -\frac{1}{2}+\frac{1}{2} i+\frac{1}{2} j-\frac{1}{2} k
\end{array}\right)
$$

Somando a terceira linha com a quarta linha da matriz anterior, teremos a terceira linha da nova matriz, equivalente a matriz inicial, resultando na matriz abaixo:

$$
\left(\begin{array}{cccc}
-\frac{1}{2}-\frac{1}{2} i+\frac{1}{2} j-\frac{1}{2} k & 1 & -1 & 0 \\
0 & -\frac{1}{2}-\frac{1}{2} i-\frac{1}{2} j-\frac{1}{2} k & 1 & -1 \\
0 & -1 & -\frac{1}{2}+\frac{1}{2} i-\frac{1}{2} j-\frac{1}{2} k & \frac{1}{2}+\frac{1}{2} i+\frac{1}{2} j-\frac{1}{2} k \\
1 & -1 & 0 & -\frac{1}{2}+\frac{1}{2} i+\frac{1}{2} j-\frac{1}{2} k
\end{array}\right)
$$

Agora, somando a primeira linha da matriz anterior com $\left(\frac{1}{2}+\frac{1}{2} i-\frac{1}{2} j+\frac{1}{2} k\right)$ multiplicado pela quarta linha da matriz anterior, obtemos a quarta linha da nova matriz abaixo:

$$
\left(\begin{array}{cccc}
-\frac{1}{2}-\frac{1}{2} i+\frac{1}{2} j-\frac{1}{2} k & 1 & -1 & 0 \\
0 & -\frac{1}{2}-\frac{1}{2} i-\frac{1}{2} j-\frac{1}{2} k & 1 & -1 \\
0 & -1 & -\frac{1}{2}+\frac{1}{2} i-\frac{1}{2} j-\frac{1}{2} k & \frac{1}{2}+\frac{1}{2} i+\frac{1}{2} j-\frac{1}{2} k \\
0 & \frac{1}{2}-\frac{1}{2} i+\frac{1}{2} j-\frac{1}{2} k & -1 & j
\end{array}\right)
$$

Multiplicando $(-i-k)$ pela terceira linha da matriz anterior e somando com a quarta 
linha da matriz anterior, obtemos a terceira linha da nova matriz. E, somando a segunda linha da matriz anterior com a quarta linha da matriz anterior, teremos a quarta linha da nova matriz, equivalente a matriz inicial. Dessa forma, a matriz obtida pelas equações citadas anteriormente é a seguinte:

$$
\left(\begin{array}{cccc}
-\frac{1}{2}-\frac{1}{2} i+\frac{1}{2} j-\frac{1}{2} k & 1 & -1 & 0 \\
0 & -\frac{1}{2}-\frac{1}{2} i-\frac{1}{2} j-\frac{1}{2} k & 1 & -1 \\
0 & 0 & k-j & -1-k \\
0 & -i-k & 0 & -1+j
\end{array}\right)
$$

Fazendo $(-i-k)$ multiplicado pela segunda linha da matriz anterior, teremos a segunda linha da nova matriz a seguir:

$$
\left(\begin{array}{cccc}
-\frac{1}{2}-\frac{1}{2} i+\frac{1}{2} j-\frac{1}{2} k & 1 & -1 & 0 \\
0 & -1+k & -i-k & i+k \\
0 & 0 & k-j & -1-k \\
0 & -i-k & 0 & -1+j
\end{array}\right)
$$

Agora, somando $(-i+j)$ multiplicado pela segunda linha da matriz anterior com $(1+j)$ multiplicado pela quarta linha da matriz anterior, obtemos a quarta linha da seguinte matriz:

$$
\left(\begin{array}{cccc}
-\frac{1}{2}-\frac{1}{2} i+\frac{1}{2} j-\frac{1}{2} k & 1 & -1 & 0 \\
0 & -1+k & -i-k & i+k \\
0 & 0 & k-j & -1-k \\
0 & 0 & -1-i-j+k & -1+i+j-k
\end{array}\right)
$$

Finalmente, multiplicando $(-1-i-j+k)$ pela terceira linha da matriz anterior e, somando com $(i+j)$ multiplicado pela quarta linha da matriz anterior, teremos a quarta linha da nova matriz como segue abaixo: 


$$
\left(\begin{array}{cccc}
-\frac{1}{2}-\frac{1}{2} i+\frac{1}{2} j-\frac{1}{2} k & 1 & -1 & 0 \\
0 & -1+k & -i-k & i+k \\
0 & 0 & k-j & -1-k \\
0 & 0 & 0 & 0
\end{array}\right)
$$

Como a matriz anterior possui posto três, segue que $\operatorname{dim}\left(\operatorname{Im} \partial_{4 q-2}\right)=3$.

- $K e r \partial_{4 q-3}$

$$
\partial_{4 q-3}=\left(\begin{array}{c}
-\frac{1}{2}-\frac{1}{2} i+\frac{1}{2} j+\frac{1}{2} k \\
-\frac{1}{2}-\frac{1}{2} i-\frac{1}{2} j+\frac{1}{2} k \\
-\frac{1}{2}+\frac{1}{2} i-\frac{1}{2} j+\frac{1}{2} k \\
-\frac{1}{2}+\frac{1}{2} i+\frac{1}{2} j+\frac{1}{2} k
\end{array}\right)
$$

Considere uma $(4 q-3)$-cadeia $\sigma$ representada por $(d, e, f, g)$, então

$$
\begin{aligned}
\partial_{4 q-3}(\sigma) & =\partial\left(d \widetilde{c}_{4 q-3,1}+e \widetilde{c}_{4 q-3,2}+f \widetilde{c}_{4 q-3,3}+g \widetilde{c}_{4 q-3,4}\right) \\
& =d\left(-\frac{1}{2}-\frac{1}{2} i+\frac{1}{2} j+\frac{1}{2} k\right) \widetilde{c}_{4 q-4,1}+e\left(-\frac{1}{2}-\frac{1}{2} i-\frac{1}{2} j+\frac{1}{2} k\right) \widetilde{c}_{4 q-4,1}+ \\
& +f\left(-\frac{1}{2}+\frac{1}{2} i-\frac{1}{2} j+\frac{1}{2} k\right) \widetilde{c}_{4 q-4,1}+g\left(-\frac{1}{2}+\frac{1}{2} i+\frac{1}{2} j+\frac{1}{2} k\right) \widetilde{c}_{4 q-4,1} \\
& =\left[d\left(-\frac{1}{2}-\frac{1}{2} i+\frac{1}{2} j+\frac{1}{2} k\right)+e\left(-\frac{1}{2}-\frac{1}{2} i-\frac{1}{2} j+\frac{1}{2} k\right)+\right. \\
& \left.+f\left(-\frac{1}{2}+\frac{1}{2} i-\frac{1}{2} j+\frac{1}{2} k\right)+g\left(-\frac{1}{2}+\frac{1}{2} i+\frac{1}{2} j+\frac{1}{2} k\right)\right] \widetilde{c}_{4 q-4,1}
\end{aligned}
$$

A $(4 q-3)$-cadeia $\sigma$ pertence ao $K e r \partial_{4 q-3}$ se, e somente se,

$$
\begin{gathered}
d\left(-\frac{1}{2}-\frac{1}{2} i+\frac{1}{2} j+\frac{1}{2} k\right)+e\left(-\frac{1}{2}-\frac{1}{2} i-\frac{1}{2} j+\frac{1}{2} k\right)+ \\
+f\left(-\frac{1}{2}+\frac{1}{2} i-\frac{1}{2} j+\frac{1}{2} k\right)+g\left(-\frac{1}{2}+\frac{1}{2} i+\frac{1}{2} j+\frac{1}{2} k\right)=0 \\
d=\frac{1}{\left(-\frac{1}{2}-\frac{1}{2} i+\frac{1}{2} j+\frac{1}{2} k\right)}\left[e\left(\frac{1}{2}+\frac{1}{2} i+\frac{1}{2} j-\frac{1}{2} k\right)+\right. \\
\left.+f\left(\frac{1}{2}-\frac{1}{2} i+\frac{1}{2} j-\frac{1}{2} k\right)+g\left(\frac{1}{2}-\frac{1}{2} i-\frac{1}{2} j-\frac{1}{2} k\right)\right]
\end{gathered}
$$


Dessa forma, temos

$$
\begin{aligned}
\sigma & =\left\{\frac { 1 } { ( - \frac { 1 } { 2 } - \frac { 1 } { 2 } i + \frac { 1 } { 2 } j + \frac { 1 } { 2 } k ) } \left[e\left(\frac{1}{2}+\frac{1}{2} i+\frac{1}{2} j-\frac{1}{2} k\right)+f\left(\frac{1}{2}-\frac{1}{2} i+\frac{1}{2} j-\frac{1}{2} k\right)+\right.\right. \\
& \left.\left.+g\left(\frac{1}{2}-\frac{1}{2} i-\frac{1}{2} j-\frac{1}{2} k\right)\right]\right\} \widetilde{c}_{4 q-3,1}+e \widetilde{c}_{4 q-3,2}+f \widetilde{c}_{4 q-3,3}+g \widetilde{c}_{4 q-3,4} \\
& =\left[\frac{1}{\left(-\frac{1}{2}-\frac{1}{2} i+\frac{1}{2} j+\frac{1}{2} k\right)}\left(\frac{1}{2}+\frac{1}{2} i+\frac{1}{2} j-\frac{1}{2} k\right) \widetilde{c}_{4 q-3,1}+\widetilde{c}_{4 q-3,2}\right] e+ \\
& +\left[\frac{\left(\frac{1}{2}-\frac{1}{2} i+\frac{1}{2} j-\frac{1}{2} k\right)}{\left(-\frac{1}{2}-\frac{1}{2} i+\frac{1}{2} j+\frac{1}{2} k\right)} \widetilde{c}_{4 q-3,1}+\widetilde{c}_{4 q-3,3}\right] f+\left[\frac{\left(\frac{1}{2}-\frac{1}{2} i-\frac{1}{2} j-\frac{1}{2} k\right)}{\left(-\frac{1}{2}-\frac{1}{2} i+\frac{1}{2} j+\frac{1}{2} k\right)} \widetilde{c}_{4 q-3,1}+\widetilde{c}_{4 q-3,2}\right] g
\end{aligned}
$$

Logo, $\operatorname{dim}\left(\operatorname{Ker} \partial_{4 q-3}\right)=3$.

Portanto, como o complexo é semi-exato, a $\operatorname{Im} \partial_{4 q-2}$ está contida no $K e r \partial_{4 q-3}$, e pelos resultados obtidos acima, temos $\operatorname{dim}\left(\operatorname{Im} \partial_{4 q-2}\right)=\operatorname{dim}\left(\operatorname{Ker} \partial_{4 q-3}\right)=3$. Dessa forma, $\operatorname{Im} \partial_{4 q-2}=\operatorname{Ker} \partial_{4 q-3}$.

- $\operatorname{Im} \partial_{4 q-3}$

$$
\partial_{4 q-3}=\left(\begin{array}{c}
-\frac{1}{2}-\frac{1}{2} i+\frac{1}{2} j+\frac{1}{2} k \\
-\frac{1}{2}-\frac{1}{2} i-\frac{1}{2} j+\frac{1}{2} k \\
-\frac{1}{2}+\frac{1}{2} i-\frac{1}{2} j+\frac{1}{2} k \\
-\frac{1}{2}+\frac{1}{2} i+\frac{1}{2} j+\frac{1}{2} k
\end{array}\right)
$$

Somando $\left(\frac{1}{2}-\frac{1}{2} i-\frac{1}{2} j-\frac{1}{2} k\right)$ multiplicado pela primeira linha, com $\left(-\frac{1}{2}-\frac{1}{2} i+\frac{1}{2} j+\frac{1}{2} k\right)$ multiplicado pela quarta linha da matriz anterior, teremos a quarta linha da seguinte matriz:

$$
\partial_{4 q-3}=\left(\begin{array}{c}
-\frac{1}{2}-\frac{1}{2} i+\frac{1}{2} j+\frac{1}{2} k \\
-\frac{1}{2}-\frac{1}{2} i-\frac{1}{2} j+\frac{1}{2} k \\
-\frac{1}{2}+\frac{1}{2} i-\frac{1}{2} j+\frac{1}{2} k \\
0
\end{array}\right)
$$

Agora, somando $\left(\frac{1}{2}-\frac{1}{2} i+\frac{1}{2} j-\frac{1}{2} k\right)$ multiplicado pela primeira linha da matriz anterior, com $\left(-\frac{1}{2}-\frac{1}{2} i+\frac{1}{2} j+\frac{1}{2} k\right)$ multiplicado pela terceria linha da matriz anterior, obtemos a terceira linha da nova matriz abaixo: 


$$
\partial_{4 q-3}\left(\begin{array}{c}
-\frac{1}{2}-\frac{1}{2} i+\frac{1}{2} j+\frac{1}{2} k \\
-\frac{1}{2}-\frac{1}{2} i-\frac{1}{2} j+\frac{1}{2} k \\
0 \\
0
\end{array}\right)
$$

Finalmente, somando $\left(\frac{1}{2}+\frac{1}{2} i+\frac{1}{2} j-\frac{1}{2} k\right)$ multiplicado pela primeira linha da matriz anterior, com $\left(-\frac{1}{2}-\frac{1}{2} i+\frac{1}{2} j+\frac{1}{2} k\right)$ multiplicado pela segunda linha da matriz anterior, obtemos a segunda linha da nova matriz abaixo:

$$
\partial_{4 q-3}\left(\begin{array}{c}
-\frac{1}{2}-\frac{1}{2} i+\frac{1}{2} j+\frac{1}{2} k \\
0 \\
0 \\
0
\end{array}\right)
$$

Como a matriz anterior possui posto um, segue que $\operatorname{dim}\left(\operatorname{Im} \partial_{4 q-3}\right)=1$

- $\operatorname{Ker} \partial_{4 q-4}$

$$
\partial_{4 q-4}=(0)
$$

Como $\partial_{4 q-4}=0$, temos $\operatorname{Ker} \partial_{4 q-4}=\mathbb{H}\left[\widetilde{c}_{4 q-4,1}\right]$, ou seja, $\operatorname{dim}\left(\operatorname{Im} \partial_{4 q-4}\right)=1$.

Portanto, como o complexo é semi-exato, a $\operatorname{Im} \partial_{4 q-3}$ está contida no $K e r \partial_{4 q-4}$, e pelos resultados obtidos acima, obtemos $\operatorname{dim}\left(\operatorname{Im} \partial_{4 q-3}\right)=\operatorname{dim}\left(\operatorname{Ker} \partial_{4 q-4}\right)=1$. Assim, $\operatorname{Im} \partial_{4 q-3}=\operatorname{Ker} \partial_{4 q-4}$.

- $\operatorname{Im} \partial_{4 q-4}$

Como $\partial_{4 q-4}$ é a aplicação nula, segue que $\operatorname{dim}\left(\operatorname{Im} \partial_{4 q-4}\right)=0$.

Novamente, como o complexo é semi-exato, a $\operatorname{Im} \partial_{4 q-4}$ está contida no $\operatorname{Ker} \partial_{4 q-1}$, e pelos resultados obtidos acima, teremos $\operatorname{dim}\left(\operatorname{Im} \partial_{4 q-4}\right)=\operatorname{dim}\left(\operatorname{Ker} \partial_{4 q-1}\right)=0 . \operatorname{Logo}$, $\operatorname{Im} \partial_{4 q-4}=\operatorname{Ker} \partial_{4 q-1}$.

Para um melhor entendimento, calculemos inicialmente a torção de Reidemeister da 
forma espacial esférica $\mathscr{P}_{3}=\frac{S^{3}}{\rho\left(P_{24}\right)}$, considerando a representação $\rho$ descrita no início da seção.

Dessa forma, temos o seguinte complexo

$$
0 \longrightarrow \mathbb{H}\left[\widetilde{c}_{3,1}\right] \stackrel{\partial_{3}}{\longrightarrow} \mathbb{H}\left[\widetilde{c}_{2,1}, \widetilde{c}_{2,2}, \widetilde{c}_{2,3}, \widetilde{c}_{2,4}\right] \stackrel{\partial_{2}}{\longrightarrow} \mathbb{H}\left[\widetilde{c}_{1,1}, \widetilde{c}_{1,2}, \widetilde{c}_{1,3}, \widetilde{c}_{1,4}\right] \stackrel{\partial_{1}}{\longrightarrow} \mathbb{H}\left[\widetilde{c}_{0,1}\right] \longrightarrow 0
$$

Pela Proposição 5.4.1, temos a exatidão do complexo acima. Assim, podemos calcular a torção de Reidemeister $\tau_{R}\left(\mathscr{P}_{3}\right)$.

Proposição 5.4.2 A torção de Reidemeister da forma espacial esférica tetraedral $\mathscr{P}_{3}$ com relação a representação $\rho: \mathbb{Z} P_{24} \rightarrow \mathbb{H}^{\times}$, definida no início da seção, é igual a

$$
\tau_{R}\left(\mathscr{P}_{3}\right)=j \in \mathbb{H}^{\times} /\left[\mathbb{H}^{\times}, \mathbb{H}^{\times}\right]
$$

Demonstração. Consideremos o complexo visto anteriormente. Escolhemos as seguintes bases para os bordos (observemos que todas elas possuem dimensão um):

$$
\begin{aligned}
& b_{3}=\left\{\widetilde{c}_{3,1}\right\} \\
& b_{2}=\left\{\widetilde{c}_{2,1}, \widetilde{c}_{2,2}, \widetilde{c}_{2,3}\right\} \\
& b_{1}=\left\{\left(-\frac{1}{2}-\frac{1}{2} i+\frac{1}{2} j+\frac{1}{2} k\right)^{-1} \widetilde{c}_{1,1}\right\} \\
& b_{0}=\emptyset
\end{aligned}
$$

Então, aplicando a construção detalhada na Seção 5.3, temos

$$
\begin{aligned}
& B_{3}=\left\{\partial_{0}\left(b_{0}\right)\right\}=\emptyset \\
& B_{2}=\left\{\partial_{3}\left(b_{3}\right)\right\}=\left\{\left(\frac{1}{2}+\frac{1}{2} i-\frac{1}{2} j-\frac{1}{2} k\right) \widetilde{c}_{2,1}+\left(\frac{1}{2}-\frac{1}{2} i+\frac{1}{2} j-\frac{1}{2} k\right) \widetilde{c}_{2,2}+\right. \\
& \left.+\left(\frac{1}{2}+\frac{1}{2} i+\frac{1}{2} j-\frac{1}{2} k\right) \widetilde{c}_{2,3}+\left(\frac{1}{2}-\frac{1}{2} i-\frac{1}{2} j-\frac{1}{2}\right) \widetilde{c}_{2,4}\right\} \\
& B_{1}=\left\{\partial_{2}\left(b_{2}\right)\right\}=\left\{\left(-\frac{1}{2}-\frac{1}{2} i+\frac{1}{2} j-\frac{1}{2} k\right) \widetilde{c}_{1,1}+\widetilde{c}_{1,2}-\widetilde{c}_{1,3},-\widetilde{c}_{1,1}+\right. \\
& \left.+\left(-\frac{1}{2}+\frac{1}{2} i-\frac{1}{2} j-\frac{1}{2} k\right) \widetilde{c}_{1,3}+\widetilde{c}_{1,4},\left(-\frac{1}{2}-\frac{1}{2} i-\frac{1}{2} j-\frac{1}{2} k\right) \widetilde{c}_{1,2}+\widetilde{c}_{1,3}-\widetilde{c}_{1,4}\right\} \\
& B_{0}=\left\{\partial_{1}\left(b_{1}\right)\right\}=\left\{\widetilde{c}_{0,1}\right\}
\end{aligned}
$$

As matrizes de mudança de base são:

$\left(B_{3} b_{3} / c_{3}\right)=[1]$ 


$$
\left(B_{0} b_{0} / c_{0}\right)=[1]
$$

As outras matrizes relevantes de mudança de base (distintas da matriz identidade) ocorrem nas dimensões 2 e 1, e serão apresentadas abaixo:

$$
\begin{aligned}
& \left(B_{2} b_{2} / c_{2}\right)=\left(\begin{array}{cccc}
\frac{1}{2}+\frac{1}{2} i-\frac{1}{2} j-\frac{1}{2} k & \frac{1}{2}-\frac{1}{2} i+\frac{1}{2} j-\frac{1}{2} k & \frac{1}{2}+\frac{1}{2} i+\frac{1}{2} j-\frac{1}{2} k & \frac{1}{2}-\frac{1}{2} i-\frac{1}{2} j-\frac{1}{2} k \\
1 & 0 & 0 & 0 \\
0 & 1 & 0 & 0 \\
0 & 0 & 1 & 0
\end{array}\right) \\
& \left(B_{1} b_{1} / c_{1}\right)=\left(\begin{array}{cccc}
-\frac{1}{2}-\frac{1}{2} i+\frac{1}{2} j-\frac{1}{2} k & 1 & -1 & 0 \\
-1 & 0 & -\frac{1}{2}+\frac{1}{2} i-\frac{1}{2} j-\frac{1}{2} k & 1 \\
0 & -\frac{1}{2}-\frac{1}{2} i-\frac{1}{2} j-\frac{1}{2} k & 1 & -1 \\
\left(-\frac{1}{2}-\frac{1}{2} i+\frac{1}{2} j+\frac{1}{2} k\right)^{-1} & 0 & 0 & 0
\end{array}\right),
\end{aligned}
$$

cujos determinantes são:

$$
\begin{aligned}
\operatorname{det}\left(B_{2} b_{2} / c_{2}\right) & =-\left(\frac{1}{2}-\frac{1}{2} i-\frac{1}{2} j-\frac{1}{2} k\right) \\
\operatorname{det}\left(B_{1} b_{1} / c_{1}\right) & =\left(-\frac{1}{2}-\frac{1}{2} i+\frac{1}{2} j+\frac{1}{2} k\right)^{-1}\left(-\frac{1}{2}+\frac{1}{2} i-\frac{1}{2} j-\frac{1}{2} k-\frac{1}{2}-\frac{1}{2} i-\frac{1}{2} j-\frac{1}{2} k+1\right) \\
& =\left(-\frac{1}{2}-\frac{1}{2} i+\frac{1}{2} j+\frac{1}{2} k\right)^{-1}(-j-k) .
\end{aligned}
$$

Dessa forma, pela definição (como o complexo é acíclico pela Proposição 5.4.1, temos $h=\emptyset$ que será omitido), identificamos a $R$ torção $\tau_{R}\left(\mathscr{P}_{3}\right)$ com o número positivo

$$
\begin{gathered}
\tau_{R}\left(\mathscr{P}_{3}\right)=\frac{\left|\operatorname{det}\left(B_{2} b_{2} / c_{2}\right)\right|}{\left|\operatorname{det}\left(B_{1} b_{1} / c_{1}\right)\right|}=\frac{\left|-\left(\frac{1}{2}-\frac{1}{2} i-\frac{1}{2} j-\frac{1}{2} k\right)\right|}{\left|\left(-\frac{1}{2}-\frac{1}{2} i+\frac{1}{2} j+\frac{1}{2} k\right)^{-1}(-j-k)\right|} \\
=\frac{\left|-\left(\frac{1}{2}-\frac{1}{2} i-\frac{1}{2} j-\frac{1}{2} k\right)\left(-\frac{1}{2}-\frac{1}{2} i+\frac{1}{2} j+\frac{1}{2} k\right)\right|}{|(-j-k)|}=\frac{|-j|}{|-j-k|} .
\end{gathered}
$$

Observemos que

$|-j-k|^{2}=(-j-k) \overline{(-j-k)}=(-j-k)(j+k)=2$.

Dessa forma, podemos simplificar a fórmula da $R$ torção do seguinte modo: 


$$
\tau_{R}\left(\mathscr{P}_{3}\right)=|-j|=j
$$

Portanto, $\tau_{R}\left(\mathscr{P}_{3}\right)=j$, ou seja, é um elemento em $\mathbb{H}^{\times} /\left[\mathbb{H}^{\times}, \mathbb{H}^{\times}\right]$. Observamos que o quatérnio $j$ possui norma 1 .

Calculemos agora, a torção de Reidemeister do caso geral, ou seja, das formas espaciais esféricas $\mathscr{P}_{4 n-1}=\frac{S^{4 n-1}}{\rho\left(P_{24}\right)}$.

Proposição 5.4.3 A torção de Reidemeister das formas espaciais esféricas tetraedrais $\mathscr{P}_{4 n-1}$ com relação a representação $\rho: \mathbb{Z} P_{24} \rightarrow \mathbb{H}^{\times}$, definida no início da seção, é igual a

$$
\tau_{R}\left(\mathscr{P}_{4 n-1}\right)=\prod_{q=1}^{n} j^{q} \in \mathbb{H}^{\times} /\left[\mathbb{H}^{\times}, \mathbb{H}^{\times}\right] .
$$

Demonstração. Consideremos o complexo visto na equação (5.4.1). Escolhemos as seguintes bases para os bordos (observemos que todas elas possuem dimensão um), com $1 \leq q \leq n$ :

$$
\begin{aligned}
& b_{4 q-1}=\left\{\widetilde{c}_{4 q-1,1}\right\} \\
& b_{4 q-2}=\left\{\widetilde{c}_{4 q-2,1}, \widetilde{c}_{4 q-2,2}, \widetilde{c}_{4 q-2,3}\right\} \\
& b_{4 q-3}=\left\{\left(-\frac{1}{2}-\frac{1}{2} i+\frac{1}{2} j+\frac{1}{2} k\right)^{-1} \widetilde{c}_{4 q-3,1}\right\} \\
& b_{4 q-4}=\emptyset
\end{aligned}
$$

Então, aplicando a construção detalhada na Seção 5.3, temos

$$
\begin{gathered}
B_{4 q-1}=\left\{\partial_{4 q-4}\left(b_{4 q-4}\right)\right\}=\emptyset \\
B_{4 q-2}=\left\{\partial_{4 q-1}\left(b_{4 q-1}\right)\right\}=\left\{\left(\frac{1}{2}+\frac{1}{2} i-\frac{1}{2} j-\frac{1}{2} k\right) \widetilde{c}_{4 q-2,1}+\left(\frac{1}{2}-\frac{1}{2} i+\frac{1}{2} j-\frac{1}{2} k\right) \widetilde{c}_{4 q-2,2}+\right. \\
\left.+\left(\frac{1}{2}+\frac{1}{2} i+\frac{1}{2} j-\frac{1}{2} k\right) \widetilde{c}_{4 q-2,3}+\left(\frac{1}{2}-\frac{1}{2} i-\frac{1}{2} j-\frac{1}{2} k\right) \widetilde{c}_{4 q-2,4}\right\} \\
B_{4 q-3}=\left\{\partial_{4 q-2}\left(b_{4 q-2}\right)\right\}=\left\{\left(-\frac{1}{2}-\frac{1}{2} i+\frac{1}{2} j-\frac{1}{2} k\right) \widetilde{c}_{4 q-3,1}+\widetilde{c}_{4 q-3,2}-\widetilde{c}_{4 q-3,3},-\widetilde{c}_{4 q-3,1}+\right. \\
\left.+\left(-\frac{1}{2}+\frac{1}{2} i-\frac{1}{2} j-\frac{1}{2} k\right) \widetilde{c}_{4 q-3,3}+\widetilde{c}_{4 q-3,4},\left(-\frac{1}{2}-\frac{1}{2} i-\frac{1}{2} j-\frac{1}{2} k\right) \widetilde{c}_{4 q-3,2}+\widetilde{c}_{4 q-3,3}-\widetilde{c}_{4 q-3,4}\right\} \\
B_{4 q-4}=\left\{\partial_{4 q-3}\left(b_{4 q-3}\right)\right\}=\left\{\widetilde{c}_{4 q-4,1}\right\}
\end{gathered}
$$

As matrizes de mudança de base são:

$$
\begin{aligned}
& \left(\begin{array}{ll}
B_{4 q-1} & b_{4 q-1} / c_{4 q-1}
\end{array}\right)=[1] \\
& \left(\begin{array}{ll}
B_{4 q-4} & b_{4 q-4} / c_{4 q-4}
\end{array}\right)=[1]
\end{aligned}
$$


As outras matrizes relevantes de mudança de base (distintas da matriz identidade) ocorrem nas dimensões $4 q-2$ e $4 q-3$, e serão apresentadas abaixo:

$$
\begin{gathered}
\left(B_{4 q-2} b_{4 q-2} / c_{4 q-2}\right)=\left(\begin{array}{cccc}
\frac{1}{2}+\frac{1}{2} i-\frac{1}{2} j-\frac{1}{2} k & \frac{1}{2}-\frac{1}{2} i+\frac{1}{2} j-\frac{1}{2} k & \frac{1}{2}+\frac{1}{2} i+\frac{1}{2} j-\frac{1}{2} k & \frac{1}{2}-\frac{1}{2} i-\frac{1}{2} j-\frac{1}{2} k \\
1 & 0 & 0 & 0 \\
0 & 1 & 0 & 0 \\
0 & 0 & 1 & 0
\end{array}\right) \\
\left(B_{4 q-3} b_{4 q-3} / c_{4 q-3}\right)
\end{gathered}
$$

cujos determinantes são:

$$
\begin{aligned}
\operatorname{det}\left(B_{4 q-2} b_{4 q-2} / c_{4 q-2}\right) & =-\left(\frac{1}{2}-\frac{1}{2} i-\frac{1}{2} j-\frac{1}{2} k\right) \\
\operatorname{det}\left(B_{4 q-3} b_{4 q-3} / c_{4 q-3}\right) & =\left(-\frac{1}{2}-\frac{1}{2} i+\frac{1}{2} j+\frac{1}{2} k\right)^{-1}\left(-\frac{1}{2}+\frac{1}{2} i-\frac{1}{2} j-\frac{1}{2} k-\frac{1}{2}-\frac{1}{2} i-\frac{1}{2} j-\frac{1}{2} k+1\right) \\
& =\left(-\frac{1}{2}-\frac{1}{2} i+\frac{1}{2} j+\frac{1}{2} k\right)^{-1}(-j-k) .
\end{aligned}
$$

Sabemos, pela Subseção 2.1.1 e pela representação $\rho: P_{24} \rightarrow \mathbb{H}^{\times}$, dada no início desta seção, que a ação do grupo $P_{24}$ sobre $S^{4 n-1}$ é dada pela representação

$$
\rho=\rho_{1} \oplus \rho_{2} \oplus \rho_{3} \oplus \cdots \oplus \rho_{n}: P_{24} \rightarrow M(2 n, \mathbb{C})
$$

Dessa forma, pela definição (como o complexo é acíclico pela Proposição [5.4.1, temos $h=\emptyset$ que será omitido), identificamos a $R$ torção $\tau_{R}\left(\mathscr{P}_{4 n-1}\right)$ com o número positivo

$$
\tau_{R}\left(\mathscr{P}_{4 n-1}\right)=\prod_{q=1}^{n} \frac{\left|\operatorname{det}\left(B_{4 q-2} b_{4 q-2} / c_{4 q-2}\right)\right|^{q}}{\left|\operatorname{det}\left(B_{4 q-3} b_{4 q-3} / c_{4 q-3}\right)\right|^{q}}=\prod_{q=1}^{n} \frac{\left|-\left(\frac{1}{2}-\frac{1}{2} i-\frac{1}{2} j-\frac{1}{2} k\right)\right|^{q}}{\left|\left(-\frac{1}{2}-\frac{1}{2} i+\frac{1}{2} j+\frac{1}{2} k\right)^{-1}(-j-k)\right|^{q}}
$$




$$
=\prod_{q=1}^{n} \frac{\left|-\left(\frac{1}{2}-\frac{1}{2} i-\frac{1}{2} j-\frac{1}{2} k\right)\left(-\frac{1}{2}-\frac{1}{2} i+\frac{1}{2} j+\frac{1}{2} k\right)\right|^{q}}{|(-j-k)|^{q}}=\prod_{q=1}^{n} \frac{|-j|^{q}}{|(-j-k)|^{q}} .
$$

Observemos que

$|-j-k|^{2}=(-j-k)(\overline{-j-k})=(-j-k)(j+k)=2$.

Assim, podemos simplificar a fórmula da $R$ torção do seguinte modo:

$$
\tau_{R}\left(\mathscr{P}_{4 n-1}\right)=\prod_{q=1}^{n}|-j|^{q}=\prod_{q=1}^{n} j^{q}
$$

Portanto, $\tau_{R}\left(\mathscr{P}_{4 n-1}\right)=\prod_{q=1}^{n} j^{q}$, ou seja, é um elemento em $\mathbb{H}^{\times} /\left[\mathbb{H}^{\times}, \mathbb{H}^{\times}\right]$. Observamos que, para cada $1 \leq q \leq n$, o quatérnio $j^{q}$ possui norma 1 . 


\section{Referências Bibliográficas}

[1] ARTIN, E. Geometric algebra. New York: Interscience Publishers, 1957.

[2] ASLAKSEN, H. Quaternionic Determinants. The Mathematical Intelligencer, v.18, n.3, p.57-65, 1996.

[3] BREDON, G.E. Topology and geometry. New York: Springer-Verlag, 1993.

[4] BROWN, K.S. Cohomology of groups. New York: Springer-Verlag, 1982.

[5] BURNSIDE, W. On a general property of finite irreducible groups of linear substitutions. Messenger of Mathematics, v.35, p.51-55, 1905.

[6] CARTAN, H.; EILEMBERG, S. Homological algebra. Princeton University Press, 1956.

[7] COHEN, M.M. A course in simple homotopy theory. New York: Springer-Verlag, 1973.

[8] DAVIS, J.F.; KIRK, P. Lecture notes in algebraic topology. Graduate Studies in Mathematics No. 35, AMS, 2001.

[9] DE RHAM, G. Complexes à automorphismes et homéomorphie différentiables, Ann. Inst. Fourier, v.2, p.51-67, 1950.

[10] FÊMINA, L. L. Grupos Split Metacíclicos e formas espaciais esféricas metacíclicas. 2011. 212 p. Tese (Doutorado em Matemática)- Instituto de Ciências Matemáticas e Computação, USP, São Carlos, 2011. 
[11] FÊMINA, L.L.; GALVES, A.P.T.; NETO, O.M.; SPREAFICO, M.F. Cellular decomposition and free resolution for split metacyclic spherical space forms, artigo aceito para publicação na revista Homotopy, homology and applications, 2013.

[12] FRANZ, W. Über die Torsion einer Überdeckung. J. Reine Angew. Math., v.173, p.245-254, 1935 .

[13] GRUEnBERG, K. Resolutions by relations. J. London Math. Soc., v.35, p.481-494, 1960.

[14] HU, S.T. Introduction to homological algebra. San Francisco: Holden-Day Series in Mathematics, 1968.

[15] MELO, T. Torção de Reidemeister das formas espaciais esféricas. 2009. 89 p. Tese (Doutorado em Matemática)- Instituto de Ciências Matemáticas e Computação, USP, São Carlos, 2009.

[16] MELO, T.; NETO, O.M.; SPREAFICO, M.F. Cellular decomposition of quaternionic spherical space forms. Geom Dedicata, v.157, DOI 10.1007/s10711-012-9714-4, 2012.

[17] MILnOR, J. Whitehead Torsion. Bulletin of the American Mathematical Society, v.72, p.358-426, 1966.

[18] MUNKRES, J.R. Elements of algebraic topology. New York: The Benjamin - Cummings Publishing Company, 1984.

[19] RAY, D.B.; SINGER, I.M. R-Torsion and the Laplacian on Riemannian Manifolds. Advances in Mathematics, v.7, p.145-210, 1971.

[20] REIDEMEISTER, K. Homotopieringe und Linseräume. Hamburger Abhandl, v.11, p.102-109, 1935.

[21] ROTMAN, J.J. An introduction to algebraic topology. New York: Springer- Verlag, 1998.

[22] ROTMAN, J.J. An introduction to the theory of groups. New York: Springer- Verlag, 1995. 
[23] STEPANOV, D.A. Gorenstein Isolated Quotient Singularities over C. arXiv: 1012.3149, v.1, 2010.

[24] SWAN, R.G. Periodic resolutions for finite groups. Annals of Mathematics, v.72, p.267-291, 1960 .

[25] TOMODA, S. Cohomology rings of certain 4-periodic finite groups. 2005. 556 p. Tese (Doctor of philosophy)- Faculty of graduate studies, University of Calgary, Alberta, 2005.

[26] TOMODA, S.; ZVENGROWSKI, P. Remarks on the cohomology of finite fundamental groups of 3-manifolds. Geometry and Topology Monographics, v.14, p.519-556, 2008.

[27] VINCENT, G. Les groupes linéaires finis sans points fixes. Commentarii Mathematici Helvetici, v.20, p.117-171, 1947.

[28] WOLF, J.A. Spaces of constant curvature. McGraw-Hill Inc., 1967.

[29] ZASSEnHAUS, H. The Theory of Groups, second edition. New York: Chelsea Publishing Company, 1958.

[30] ZIMMERMANN, B. On the classification of finite groups acting on homology 3-spheres. Pacific Journal of Mathematics, v.217, n.2, p.387-395, 2004. 



\section{Índice Remissivo}

$\operatorname{Ext}(A, B), 103$

$\operatorname{Hom}(A, B), 102$

p-grupo, 25

$p$-subgrupo de Sylow, 26

$p q$-condição, 26

álgebra dos quatérnios, 131

anel

de cohomologia, 105

grupo, 20

aplicação

aumentação, 20

de cadeias, 115

diagonal, 127

complemento, 21

curved join, 37

espaço representação, 22

forma espacial esférica, 25

tetraedral, 36

função determinante, 130

G-espaço topológico, 20

livre, 20

trivial, 20 grupo

ação, 19

binário tetraedral, 33

de Whitehead, 130

de Whitehead especial, 130

de Whitehead reduzido, 131

linear especial, 130

linear geral, 129

livre de ponto fixo, 24

ortogonal, 23

unitário, 23

homotopia

contrátil, 115

de cadeias, 115

módulo

de homomorfismos, 102

trivial, 20

produto

cup, 104, 128

direto, 21

semidireto, 21

realiza, 21 
região fundamental, 39

representação, 22

absolutamente irredutível, 23

complexa, 23

equivalente, 22

fiel, 24

grau, 22

irredutível, 22

livre de ponto fixo, 24

ortogonal, 23

ortogonalmente equivalente, 23

real, 23

totalmente irredutível, 22

unitária, 23

unitariamente equivalente, 23

resolução, 20

canônica livre, 103

livre, 20

projetiva, 20

subrepresentação, 22

própria, 22

teorema

da dualidade de Poincaré, 105

dos coeficientes universais, 104

torção de Reidemeister, 134 